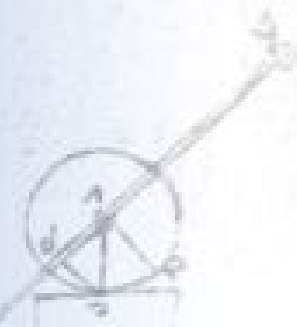

\title{
28
}

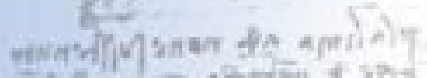

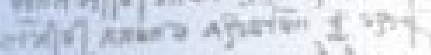

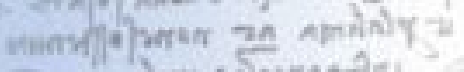

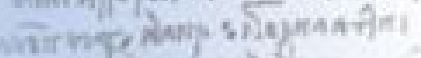

\section{Francesco Capone}

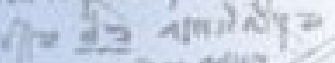

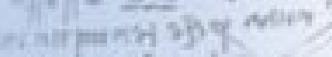

\section{I sistemi locali turistici in Italia}

Identificazione, misurazione ed analisi delle fonti di competitivita
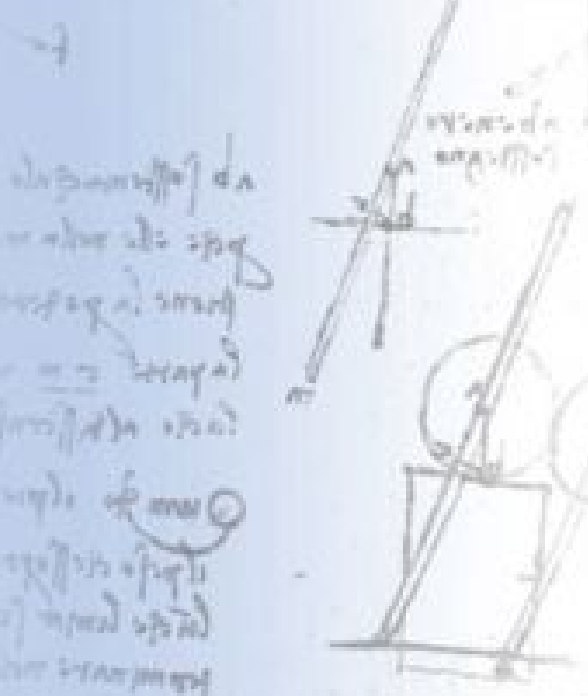
TESI

SCIENZE SOCIALI

$-1-$ 
TESI

SCIENZE Sociali

1. Francesco Capone, I sistemi locali turistici in Italia. Identificazione, misurazione ed analisi delle fonti di competitività , 2005 
UNIVERSITÀ DEGLI STUDI DI FIRENZE, FACOLTÀ DI ECONOMIA DOTTORATO IN ECONOMIA E GESTIONE DELLE IMPRESE

E DEI SISTEMI LOCALI - XVII ${ }^{\circ}$ CICLO

\title{
I SISTEMI LOCALI TURISTICI IN ITALIA
}

IDENTIFICAZIONE, MISURAZIONE ED ANALISI DELLE FONTI DI COMPETITIVITÀ

\author{
Tesi di Dottorato di \\ Francesco Capone
}

Tutor: Prof.ssa Luciana Lazzeretti 
I sistemi locali turistici in Italia : identificazione, misurazione ed analisi delle fonti di competitività / Francesco Capone. - Firenze : Firenze university press, 2005.

(Tesi. Scienze Sociali, 1)

Edizione elettronica disponibile su http://e-prints.unifi.it

Stampa a richiesta disponibile su http://epress.unifi.it

ISBN 88-8453-321-X (online)

ISBN 88-8453-322-8 (print)

338.4791 (ed. 20)

Turismo - Economia

(C) 2005 Firenze University Press

Università degli Studi di Firenze

Firenze University Press

Borgo Albizi, 28

50122 Firenze, Italy

http://epress.unifi.it/

Printed in Italy 
A Carlo, Tina e Riccardo...

... "Schin'ichi, un giorno o l'altro dovremo fondare un'università", aveva detto Toda un bel giorno. "L'aveva già prospettata Makiguchi questa idea, se non ci riesco io, nel tempo che mi resta da vivere, bisognerà che ci pensi tu. Dopo tutto la pace ha per fondamento proprio l'educazione"...

D. Ikeda, La nuova Rivolurione Umana, vol. I, 2001, p.154. 

Desidero esprimere la mia gratitudine a tutti coloro che mi hanno aiutato nella raccolta, elaborazione e realizzazione di questo lavoro.

Un primo ringraziamento va al dott. Stefano Menghinello, il cui aiuto è stato fondamentale per identificare e mappare i sistemi locali turistici italiani, durante il mio primo soggiorno di studio presso l'ISTAT di Roma al Dipartimento Statistiche Strutturali (DSS) nei mesi di Febbraio-Aprile 2004.

Desidero, poi, ringraziare il Prof. Joan Trullen Thomas, docente di Economia Urbana ed il suo collaboratore dott. Rafael Boix Domenech del Dipartimento di Economia Aplicada (DEA) di Barcellona, per avermi introdotto alle teorie relative alla "economia delle reti di citta" ed alle metodologie econometriche di statistica spaziale. Senza il loro aiuto nel corso del mio secondo soggiorno di studio (Maggio-Luglio, Novembre 2004) presso l'Universidad Autonoma di Barcellona (UAB), l'analisi delle fonti di competitività dei SLT italiani non sarebbe stata svolta.

Un sentito grazie va inoltre al Prof. Fabio Sforzi, dell'Università di Parma per i consigli che mi ha dato in ordine alla identificazione geografica dei SLT italiani; al Prof. Marco Bellandi ed alla Prof.ssa Gabi Dei Ottati del Dipartimento di Economia dell'Università di Firenze, per aver discusso con me alcune questioni relative alla estensione dell'approccio distrettuale ai sistemi turistici; ed alla Prof.ssa Alessandra Petrucci del Dipartimento di Statistica dell'Università di Firenze per avermi aiutato nella applicazione del programma Arcview.

Inoltre, un ringraziamento particolare va al mio supervisor, la Prof.ssa Luciana Lazzeretti dell'Università di Firenze, la cui guida e consiglio non è mai mancato e che ha sempre cercato, come un maestro dovrebbe fare, di aiutarmi ad esprimere il meglio di me.

Ringrazio, infine gli amici i quali, anche se distanti dall'argomento di questa tesi, mi sono stati vicini e mi hanno incoraggiato costantemente. La riuscita di questo lavoro è merito anche loro. scrive.

Naturalmente la responsabilità di quanto scritto resta interamente a carico di chi 



\section{INDICE}

INTRODUZIONE

PRIMA PARTE

\section{CAPITOLO I}

IL “SISTEMA LOCALE TURISTICO” NEL QUADRO TEORICO DI RIFERIMENTO

1.2. I modelli sistemici per il turismo di matrice aziendale: un richiamo al DM ed allo SLOT 20

1.2.1. Il Destination Management

1.2.2. Il Sistema Locale di Offerta Turistica (SLOT) 22

1.3. I Modelli territoriali di origine economico-industriale applicati al turismo 23

1.3.1. Il Sistema Territoriale Locale 23

1.3.2. Il Milieu turistico 24

1.3.2. Il Cluster Turistico

1.3.4. Il Distretto Turistico 26

1.4. Il modello di riferimento: il sistema locale turistico (SLT) 29

1.4.1. Il punto di partenza: l'approccio distrettuale 29

1.4.2. Alcuni concetti di base: il PACA ed il cluster di attori 30

1.4.3. Il Sistema Locale Turistico 31

1.4.4. La dimensione multiscala territoriale del SLT 33

1.5. STL e la legge 135/2001 35

1.7. Conclusioni 38

\section{CAPitolo II}

IDENTIFICAZIONE ED ANALISI DEI SISTEMI LOCALI TURISTICI IN ITALIA 41

2.1. Premessa 41

2.2. Lo stato dell'arte: l'individuazione dei SLT in italia 42

2.3. La fonte dei dati 47

2.3.1. L'unità di analisi territoriale: i sistemi locali del lavoro 49

2.4. Primo studio: Il censimento intermedio del 1996 ed il settore Horeca 51

2.4.1. I risultati del primo studio 53

2.5. Secondo studio: Il Censimento del 2001 ed la filiera turistica 56

2.5.1. Una breve analisi della domanda turistica 65

2.5.2. Una Cluster Analysis sui SLT 69

2.5.3. Una classificazione per analizzare i SLT 71

2.6. Conclusioni 73 


\section{PARTE SECONDA}

\section{CAPITOLO III}

LE FONTI DELLA COMPETITIVITÀ DEI SISTEMI PRODUTTIVI LOCALI: RISORSE NATURALI, ECONOMIE ESTERNE E SPILLOVERS

3.1. Premessa

3.2. La dotazione di risorse naturali 78

3.3. Cenni sulle economie di localizzazione 81

3.4. Il contributo dell'Economia Urbana e Regionale $\quad 82$

3.4.1. Le economie di agglomerazione 82

3.4.2. Le economie di rete 83

3.4.3. Spillovers spaziali 84

3.5. Una proposta di classificazione 86

\section{CAPITOLO IV}

LA METODOLOGIA UTILIZZATA: L’ECONOMETRIA SPAZIALE 87

4.1. Premessa

4.2. La dipendenza Spaziale e l'Eterogeneità

4.3. Alcuni cenni storici sull'Econometria Spaziale 91

4.4. I test di effetti spaziali 93

4.4.1. I test univariati spaziali locali e globali 93

4.4.2. I test locali di dipendenza spaziale $\quad 95$

4.5. Le analisi multivariate di dipendenza spaziale 97

\section{CAPitolo V}

ANALISI E MISURAZIONE DELLE FONTI DI COMPETITIVITÀ DEI SLT 101

5.1. La misurazione delle economie esterne e spatial spillovers 101

5.1.1. Premessa 101

5.1.2. Il framework teorico di analisi delle economie esterne: un modello knowledge-based 102

5.2. Analisi delle fonti della competitività nei SLT. Un primo studio: le città come unità di analisi

5.2.1. Dati e Variabili 103

5.2.2. Modelli non spaziali 105

5.2.3. Modelli spaziali 106

5.2.4. Regimi Spaziali 106

5.2.5. Conclusioni del primo studio 111

5.3. Analisi delle fonti della competitività nei SLT. Un secondo studio: i sistemi locali come unità di analisi 
5.3.1. Dati e Variabili

5.3.2. Modelli non spaziali

5.3.3. Modelli spaziali

5.3.4. Regimi Spaziali

5.3.5. Conclusioni del secondo studio

5.4. Conclusioni

RIFLESSIONI CONCLUSIVE

RIFERIMENTI BIBLIOGRAFICI

INDICE DELLE FIGURE

INDICE DELLE TABELLE

APPENDICI 


\section{INTRODUZIONE}

L'obiettivo di questa tesi si può riassumere principalmente in un tentativo di identificazione, misurazione ed analisi dei sistemi locali turistici (SLT) italiani, realizzato utilizzando un approccio integrato fra economia distrettuale ed economia delle reti di città, l'applicazione della metodologia Istat-Sforzi prima e le tecniche di econometria spaziale dopo.

I cambiamenti socio-economici dell'ultimo decennio hanno modificato e trasformato sostanzialmente l'ambiente competitivo delle imprese in tutti i settori economici. Il processo di globalizzazione ha reso il pianeta più piccolo e le risorse a basso costo sono maggiormente disponibili agli operatori economici. In uno scenario di questo tipo tuttavia la competizione di nazioni e di imprese è sempre più legata a vantaggi locali. Le principali industrie mondiali sono localizzate in alcuni luoghi specifici: si pensi alla Silicon Valley, alle apparecchiature bio-mediche nell'area di Boston o all'esperienza distrettuale in Italia.

A fronte di questo mutamento gli studiosi di economia hanno dovuto modificare ed ampliare il loro punto di vista e lo studio della competizione è traslato dall'analisi delle imprese e delle relazioni fra imprese appartenenti allo stesso settore, all'analisi delle imprese relative allo stesso sistema locale e tra sistemi locali differenti.

Questo stesso ragionamento, che ha avuto origine nell'ambito dei settori cosiddetti "industriali/manifatturieri", può essere esteso facilmente all'industria dei servizi in generale ed a quella "turistica" in particolare, tanto più che le risorse artistiche, culturali ed ambientali di una destinazione sono "localizzate" e non mobili e che la competizione si sviluppa tra luoghi e non tra singoli attori economici.

È evidente come, nella società attuale, l'industria turistica rappresenti un importante settore economico in crescita. Secondo le statistiche diffuse dal World Tourism Organization (WTO) ${ }^{1}$ il movimento degli arrivi, a livello mondiale, ha superato, nel 2002, la quota 700 milioni di unità; cifra significativa di un fenomeno economico, sociale, culturale e politico in ascesa. Il conseguente movimento monetario è ugualmente in crescita, in quanto è passato dai 2 miliardi di dollari del 1950 a ben 475 miliardi nel 2002. Il turismo negli ultimi decenni è diventato uno dei principali settori dell'economia mondiale. Gli stati pongono sempre più attenzione alle opportunità che offre questa attività.

Dato questo scenario di riferimento, in prima approssimazione, lo scopo di questa tesi è principalmente rispondere a due domande di tipo generale:

1. Quali sono i luoghi turistici in Italia?

2. In che modo competono questi luoghi tra loro?

Per poi concentrarci su una unica unità di analisi: il "sistema locale turistico".

Riguardo al primo quesito, ci siamo chiesti quali sono i sistemi di produzione turistica in Italia e come fare per individuarli. Abbiamo effettuato un'identificazione dei sistemi locali turistici italiani tramite un approccio agli attori ed alla struttura dell'offerta. In relazione alla seconda domanda, ci siamo interrogati su come competono questi 1 Tourism Highlights Edition 2003, World Tourism Organization, 2003. disponibile su
http://www.world-tourism.org/market_research/facts/highlights/Highlights.pdf 
sistemi e quali sono i loro vantaggi. In particolare, in base all'identificazione dei sistemi locali turistici, abbiamo analizzato e misurato i vantaggi delle imprese che fanno parte di questi sistemi (economie esterne e spillovers). Le due parti in cui è articolata la tesi seguono questo schema di ragionamento.

Nella prima parte relativa alla identificazione e misurazione dei SLT italiani, si passano in rassegna i modelli sistemici sul turismo con particolare attenzione a quelli di origine aziendale ed economico-industriale, estesi poi all'industria turistica. Il panorama proposto è necessario per introdurre il concetto di "sistema locale turistico" (SLT), utilizzando come punto di partenza e riflessione la teoria distrettuale. Si individuano i sistemi locali turistici che fino ad oggi hanno ricevuto una scarsa attenzione, servendosi di un approccio di tipo classico e sistemico tramite la metodologia Istat-Sforzi che individua la struttura dell'offerta turistica a partire dai sistemi locali del lavoro (SLL).

In particolare, nel primo capitolo, tralasciando gli studi settoriali sul turismo (alberghiero, trasporti, attività ricreative), ci siamo concentrati prevalentemente sulle analisi ad approccio sistemico che esaminano "un luogo come un sistema di attori specializzati in attività turistiche". Abbiamo suddiviso i contributi in base all'origine disciplinare: prima quelli di matrice economico-aziendale e poi quelli di natura economico-industriale.

Con il secondo capitolo si configura una prima identificazione e mapping dei sistemi turistici italiani basata sui SLL. Si propone un approccio "diretto" al turismo, per poi risalire alle relazioni con le altre attività interrogandoci su quali siano i sistemi locali turistici in Italia e quali le loro attività tipiche, se esista una filiera turistica generalizzabile ed infine, se i sistemi locali turistici individuati siano rappresentativi delle destinazioni turistiche. Si indicano alcune soluzioni a tali problemi riflettendo sulla metodologia utilizzata e sull'applicabilità all'industria turistica e configurando la variabilità dei risultati rispetto alle diverse definizioni di turismo prese in considerazione.

Nella seconda parte, la prospettiva classica si arrichisce con gli studi relativi all'econometria spaziale e con le teorie delle reti di città e degli spillovers spaziali. La dimensione dei SLL è quindi ampliata con gli spillovers tra SLL limitrofi, reti di destinazioni turistiche (città) e reti di sistemi locali turistici. Si passano in rassegna operativamente i vantaggi delle imprese nei sistemi di produzione (economie esterne) e se ne propone una classificazione. Un capitolo è poi dedicato alla presentazione della metodologia applicata: l'econometria spaziale. Nella parte finale si misurano le economie esterne e gli spillovers spaziali per i sistemi locali turistici italiani.

Il terzo capitolo in particolare analizza le fonti della competitività dei sistemi produttivi locali. L'attenzione è posta sul difficile quesito in merito a quali siano le motivazioni dell'esistenza di concentrazioni di imprese; la competitività delle imprese proviene da una rilevante presenza di risorse naturali nel territorio o è generata dalle economie esterne? La prima parte di questo capitolo passa in rassegna i contributi in cui le risorse naturali sono considerate come fonti della concentrazione di imprese. Successivamente sono presentate le economie esterne di localizzazione, urbanizzazione e rete. I riferimenti teorici sono sintetici per giungere alla questione che qui più interessa, ovvero una tassonomia utile per la "misurazione e analisi dei differenziali di competitività di un luogo". Abbiamo, inoltre, cercato di ampliare le nostre strumentazioni teoriche ed empiriche per analizzare le diverse performance di destinazioni turistiche, spesso costituite da città o da gruppi di città. A questo scopo 
vengono fatti dei richiami alla teoria delle reti di città e si prospetta la necessità di allargare l'analisi a destinazioni limitrofe e a reti di località turistiche.

Il quarto capitolo espone i fondamenti metodologici dell'analisi sugli spillovers spaziali. Si illustra l'econometria spaziale à la Anselin $(1988,1992)$ che sarà poi la base della applicazione empirica. In che modo si può analizzare il vantaggio competitivo di un luogo presupponendo che il suo rendimento è relazionato anche a quello delle località limitrofe (attrattività, infrastrutture, etc.)? L'econometria spaziale ci aiuta a risolvere questo problema.

Il quinto capitolo presenta i modelli econometrici di stima delle economie esterne e degli spillovers dei sistemi locali turistici identificati. Le diverse performance dei SLT (ad esempio la crescita dell'occupazione) sono misurate in rete tra network di città (primo studio) e tra network di sistemi locali (secondo studio). Verifichiamo così, se esiste un legame tra la crescita occupazionale di una destinazione turistica e le performance (occupazione, popolazione, infrastrutture) dei luoghi vicini.

Infine, si propongono alcune risposte ed approfondimenti alle domande formulate e alcune riflessioni conclusive.

Lo schema teorico di analisi è presentato nella figura seguente. 


\section{LO SCHEMA TEORICO DI ANALISI}

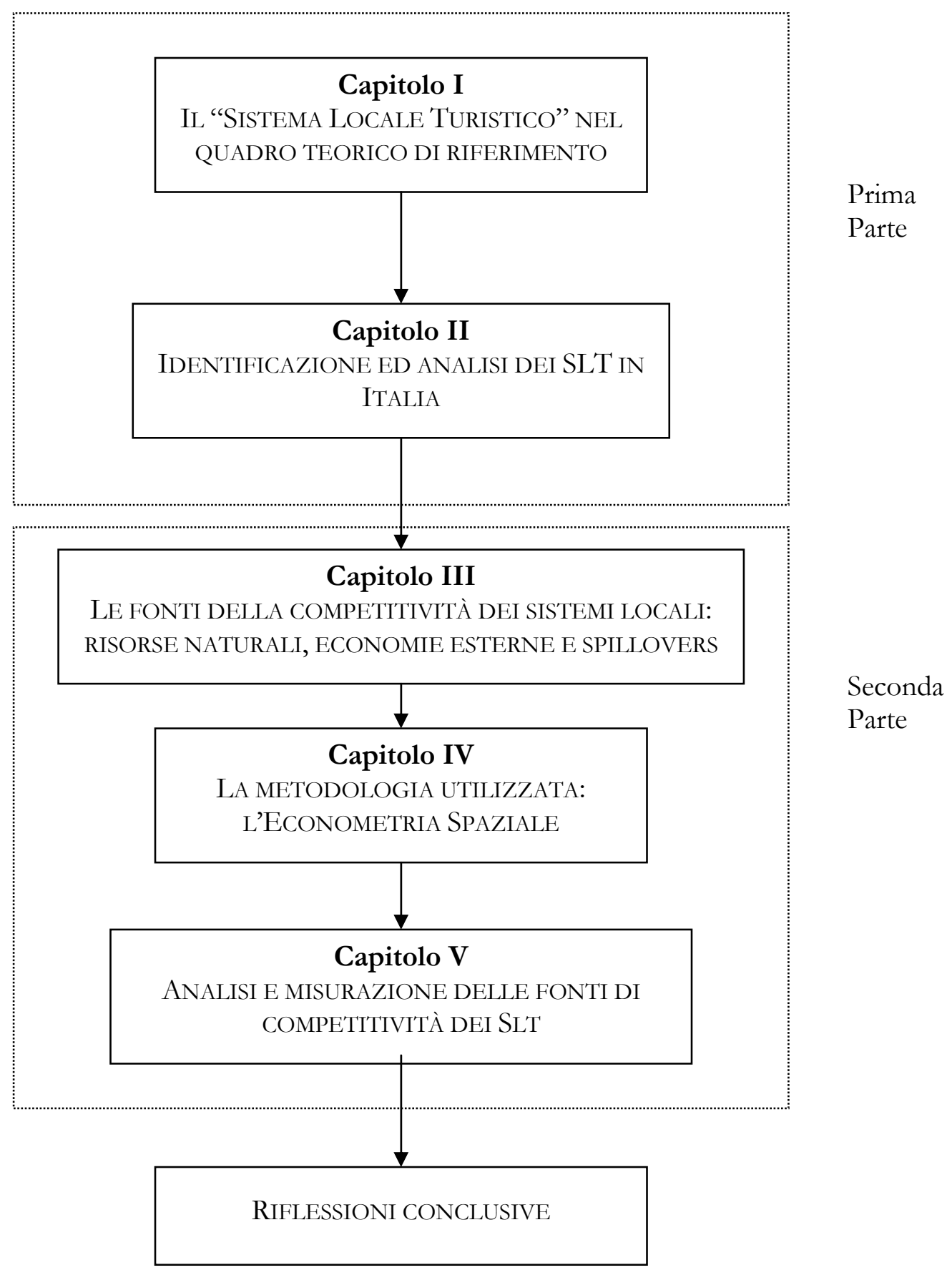


PRIMA PARTE 


\section{CAPITOLO I}

\section{IL “SISTEMA LOCALE TURISTICO” NEL QUADRO TEORICO DI RIFERIMENTO}

\subsection{Premessa}

La Commissione Europea ha evidenziato la sfida rivolta a migliorare la qualità della vita nelle regioni e nei luoghi del territorio comunitario, garantendo al contempo un'alta competitività e promuovendo lo sviluppo sostenibile. È quindi di fondamentale importanza lo studio di nuove ipotesi di sviluppo locale, che implichi una riflessione sulle risorse disponibili nel territorio da conservare e valorizzare, in grado di innescare processi riproduttivi e sostenibili nel tempo.

È evidente come, nella società attuale, l'industria dei viaggi e del turismo rappresenti un importante settore economico in crescita. Secondo le statistiche diffuse dal World Tourism Organization (WTO) ${ }^{2}$ il movimento degli arrivi, a livello mondiale, ha superato, nel 2002, la quota di 700 milioni di unità; cifra significativa di un fenomeno economico, sociale, culturale e politico in ascesa. Il conseguente movimento monetario è ugualmente in crescita, in quanto passato dai 2 miliardi di dollari del 1950 a 475 miliardi nel 2002. Il turismo negli ultimi decenni si è convertito in uno dei principali settori dell'economia mondiale.

Gli stati Europei sono caratterizzati indubbiamente da una diffusa dotazione di patrimonio artistico, culturale ed ambientale che è la base della propria offerta turistica. Questo patrimonio se posto al centro di valide strategie di valorizzazione e conservazione economica, può creare un vantaggio competitivo sostenibile e duraturo.

Lo scopo di questo capitolo è di approfondire le tematiche dello sviluppo locale di un luogo con attrazioni culturali ed ambientali e di presentare un sistema di imprese turistiche come un motore di sviluppo, analizzando le sue specifiche caratteristiche e differenze.

Si passa in rassegna quella parte di letteratura scientifica che analizza il turismo concentrandosi su approcci di tipo sistemico e si tralascia gli studi meramente settoriali (alberghiero, trasporto, ristorazione, etc.). Nel primo paragrafo si propone un panorama dei contributi relativi ai modelli sistemici di tipo aziendale (Destination Management, SLOT), mentre nel secondo quelli di origine industriale ${ }^{3}$ poi estesi all'industria turistica (cluster, milieu, distretto). Infine si presenta un modello il Sistema Locale Turistico (SLT) come chiave di lettura di un sistema di attori specializzati in attività turistiche ed istituzioni associate, utilizzando come punto di partenza l'approccio distrettuale.

\footnotetext{
2 Tourism Highlights Edition 2003, World Tourism Organization, 2003. Disponibile su http://www.worldtourism.org/market_research/facts/highlights/Highlights.pdf

${ }^{3}$ Per un'analisi dei modelli territoriali di origine industriale si veda il contributo di Moulart e Sekia (2003) e il report della Commissione Europea (2003) e Bergman e Fesser (1999).
} 
Lo scopo è di definire il concetto di "sistema (locale) di imprese turistiche", avendo cura di chiarirne la dimensione territoriale e le caratteristiche socioculturali specifiche radicate nella realtà locale. Si fa poi un breve riferimento alla legge 135/2001 che introduce nell'articolo cinque i sistemi locali turistici. Vedremo che cosa sono questi sistemi e quale riconoscimento hanno avuto in Italia. Il capitolo si conclude con l'analisi del problema dei confini geografici nella definizione di una destinazione turistica (o di un sistema locale) e come questi confini spesso siano meramente amministrativi ed artificiosi.

\subsection{MODELLI SISTEMICI PER IL TURISMO DI MATRICE AZIENDALE: UN}

\section{RICHIAMO AL DM ED ALLO SLO'T}

\subsubsection{Il Destination Management}

L'importanza di un approccio di tipo sistemico al turismo nell'ambito degli studi di origine aziendale è emerso da circa un decennio. In particolare, la letteratura internazionale si rifà principalmente al concetto di "Destination", concentrando l'attenzione sulle strategie di gestione e marketing di un luogo identificato da un sistema di attori che cooperano al fine di fornire un prodotto turistico integrato. La Commissione Europea definisce una destinazione turistica (Tourist Destination): "as an area which is separately identified and promoted to tourists as a place to visit, and within which the tourist product is coordinated by one ore more identifiable authorities or organisations" (E.C., 2000:149) ${ }^{4}$.

La Commissione descrive l'importanza della percezione del turista in una località e come sia necessario avere un approccio sistemico ed integrato alla gestione ed alla qualità: "As far as tourists are concerned, however, the satisfaction derived from staying at a destination does not just depend on their experience of tourist services, but also on more general factors such as hospitality, safety and security sanitation and salubrity, traffic and visitor management" (ibidem:13).

Infatti, si richiama il ruolo strategico dell'approccio sistemico al fine di percorrere linee di sviluppo virtuose coinvolgendo non solo i visitatori, ma anche gli attori economici (internamente e esternamente alla località), la comunità locale e i suoi rappresentati.

Il turismo è definito come un prodotto astratto, frammentato, diversificato che richiede la partecipazione e la collaborazione di molti anelli della filiera (tour operator, travel agents, alberghi ed altri servizi). I visitatori ricevono una serie di stimoli provenienti dalla località turistica, ma che non sono specificatamente "destinati" a loro: si pensi alla sicurezza, allo stato delle strade, all'inquinamento, ai vari servizi al cittadino (uffici postali, telefoni, banche, trasporti, etc.).

La Destination viene descritta come un luogo caratterizzato da due elementi; il primo è la realtà interna: un ambito territoriale coerente in cui un gruppo di attori lavora insieme e sviluppa le proprie decisioni; la seconda è la realtà esterna, basata sulla immagine e sulla percezione esterna, cioè il significato e l'immagine per i turisti.

Il concetto del Destination Management (DM) si è sviluppato con i principali contributi

\footnotetext{
${ }^{4}$ Un'area che risulta separatamente identificata e promossa ai turisti come un luogo da visitare e nel quale il prodotto turistico è coordinato da una o più autorità od organizzazioni identificabili. Traduzione propria.
} 
di Ritchie, Laws, Buhalis e dei membri dell'AIEST ${ }^{5}$ : Bieger, Keller, Pechlaner, Weiermaier, ed analizza le località turistiche come un insieme di attori localizzati in un luogo e considera la destinazione come un set di prodotti in grado di attrarre visitatori. In particolare, in Pechlaner e Weiermaier (2000) uno sguardo approfondito viene sviluppato principalmente sul governo della destinazione, su come gestire le forme di finanziamento, la sua organizzazione, le sue competenze e risorse umane e le diverse strategie di marketing. Ad esempio, Bieger (1998:7) definisce una destination come "as areas consist of all services and offers a tourist consumers during his/ her stay".

Inoltre dato che il turismo viene definito come una amalgama eterogenea di servizi (Ritchie 1999; Buhalis, 2002) dal punto di vista del consumatore, il viaggio non è più un servizio od un prodotto, ma si trasforma sempre di più in un'esperienza (Asworth, 1991). Nella letteratura sistemica sul turismo ci si riferisce alle relazioni tra gli attori di un luogo in base ad un concetto la coopetition (Edgell e Haenisch, 1995), come la cooperazione e la competiz̧ione. Il focus è quindi spostato sulla rilevanza e sul ruolo di queste due forze che risultano essenziali in una destinazione come in altri modelli territoriali (distretto o cluster).

Questa vicinanza teorica con i modelli territoriali è evidenziato anche nel lavoro di Crouch e Ritchie (1998); gli autori presentano le note cinque forze di Porter per analizzare la competitività di una località turistica ${ }^{6}$ e questo si configura come un prototipo di cluster turistico.

Uno studio simile viene, per esempio, effettuato da Buhalis (2001). L'autore analizza le destinazioni come un amalgama di prodotti turistici che offrono un'esperienza integrata ai consumatori. Inoltre focalizza come il ruolo degli attori sia spesso interdipendente, come "il sistema di offerta" dipenda da ognuno degli attori coinvolti ${ }^{7}$ ed infine come molte delle imprese del comparto turistico, piccole e medie, siano caratterizzate da questa interdipendenza (Buhalis e Cooper, 1999).

L'approccio DM si è sviluppato anche in Italia (Franch, 2002; Tamma 1999; Martini, 2002; Sainaghi et al. 2003) e recentemente è stato al centro di un fervido dibattito. Franch (2002) suddivide l'approccio ad una destinazione dal punto di vista dell'offerta "supply-side" (SLOT) e dal punto di vista della domanda "demand-side" (DM). Nel DM la destinazione si configura come un insieme di prodotti e servizi che si sviluppano come risorse per attrarre il turista, nello SLOT si individua invece un sistema correlato di offerta relativo ad un determinato territorio, distinguendolo quindi dal prodotto turistico (Franch, 2002:3).

In questa ottica alcuni autori italiani (Franch, 2002:2) si interrogano su tre principali aspetti: a) la definizione della destinazione come entità a cui riferire le scelte strategiche e le politiche di governo turistico; b) le forme di destination management e c) le politiche alternative al destination management. Abbiamo già accennato agli approcci alla destinazione dal lato della domanda e dal lato dell'offerta, che si riferisce all'analisi dei segmenti da attrarre o alla composizione dell'offerta locale, ci si interroga poi sul

\footnotetext{
5 International Association of Scientific Experts in Tourism, www.aiest.org, di cui ben tre convegni internazionali furono dedicati al "Destination Management and Marketing" (1996, 1997, 1998) (Pechlaner and Weiermaier, 2000:4).

${ }^{6}$ Gli autori propongono un modello di rilevante interesse per aumentare la competitività di un luogo tramite un modello micro e macro economico di attori. Questo lega le risorse ai vantaggi competitivi e comparativi raggiungibili, che potrebbe essere interpretato come una forma elementare di cluster porteriano. Nonostante l'unità di analisi, quindi sia un sistema locale, il focus si sviluppa maggiormente sugli aspetti manageriali di strategia, marketing e gestione.

${ }^{7}$ Buhalis evidenzia, inoltre, come spesso un'esperienza sia relazionata al nome della località come se fosse una marca (Leiper, 1995).
} 
coordinamento delle decisioni, dove il problema principale è quello relativo al processo decisionale a livello degli attori locali, sia al livello di popolazioni sia di destinazioni concorrenti.

In questa ottica, Franch (2002:7) evidenzia che i possibili percorsi per risolvere tali problematiche sono a) l'individuazione di un soggetto in grado di individuare ed orientare il comportamento della destinazione; b) la promozione da parte di un organizzazione pubblica in cooperazione con gli attori locali, ed infine c) la necessità di auto-regolamentare $i$ soggetti in funzione dei due punti precedenti per perseguire un orientamento di fondo della località. Inoltre, le relazioni tra questo approccio e quelli più tipicamente italiani si sono sviluppate sostanzialmente con particolare attenzione al modello SLOT che tratteremo nel prossimo paragrafo.

\subsubsection{Il Sistema Locale di Offerta Turistica (SLOT)}

Nella letteratura italiana si è diffuso una decina di anni fa l'approccio di tipo sistemico al turismo riferito principalmente agli studi di Rispoli e Tamma ed al concetto di SLOT. L'approccio sistemico applicato ad una località turistica analizza i fattori di attrattività e l'insieme di attività localizzate. Questo si basa su un forte legame tra l'offerta delle aziende e l'offerta complessiva di una località e richiama esplicitamente la definizione di Sistema Locale di Offerta Turistica (SLOT): "un insieme di attività e fattori di attrattività che situati in uno spazio definito (sito, località, area), siano in grado di proporre un'offerta turistica articolata e integrata, ossia rappresentino un sistema di ospitalità turistica specifica e distintiva che valorizza le risorse e la cultura locali" (Rispoli e Tamma, 1995:41).

Lo SLOT è caratterizzato da un insieme di quattro elementi:

a. Sistema: un insieme di attività integrate fra loro e con il territorio che richiedono un coordinamento e il coinvolgimento da parte di tutti gli attori;

b. Locale: il riferimento ad uno spazio definito che determina le caratteristiche fondamentali e peculiari, la cultura e i fattori di attrattività specifica;

c. Offerta Turistica: il fine del sistema è appunto quello di offrire una gamma di prodotti turistici;

d. Sistema Locale di Offerta (e non un Sistema di Offerta Locale) che definisce l'area come un sistema aperto che si connette con l'esterno.

Questi elementi sono la base per valutare la capacità di un luogo di produrre ricchezza, occupazione e verificare il suo potenziale ad innescare cicli virtuosi di sviluppo. Inoltre, vedremo che questi elementi costituiscono i fondamenti comuni per l'analisi dei modelli industriali estesi all'industria turistica.

Entrambi i modelli presentati (DM e SLOT) elencano elementi diversi, ma continuano a richiamare l'attenzione sugli attori locali e i loro legami, sulle risorse del luogo e sulle relazioni tra il sistema locale e quello sovra-locale?

\footnotetext{
8 Alcune applicazioni del SLOT applicati ad alcune aree del mezzogiorno si possono trovare tra i progetti di Sviluppo Italia e in un'applicazione in Sicilia in AA.VV. (2001).

${ }^{9}$ La differenza sostanziale di questo approccio con i modelli territoriali che tratteremo successivamente è che la gestione del luogo e la pianificazione, sono ruoli affidati ad un singolo soggetto leader, ad un meta-manager, al quale viene delegata, non solo la gestione, ma anche la programmazione strategica del sistema. Questo aspetto, risulta differente in quei luoghi, per esempio, gli agglomerati di piccola e media impresa, dove non
} 
Una parte della letteratura specialistica nazionale, partendo dal contributo dello SLOT, ha posto attenzione agli approcci sistemici all'industria turistica (Casarin, 1999; Tamma, 1999; Della Corte, 2000). Ad esempio, Casarin (1999) ne sottolinea gli aspetti essenziali nella gestione diretta del territorio attraverso la partecipazione ai processi decisionali di sviluppo degli attori locali.

Tamma sviluppa invece un collegamento dello SLOT con il DM tramite due aspetti correlati. Innanzitutto riflettendo sulla gestione del portafoglio di prodotti turistici offerti sul mercato ed in seconda fase sulle risorse/attività/attori, che costituiscono il sistema locale di offerta. Lo stesso autore rileva come la DM sia il tentativo di promuovere e organizzare l'integrazione dell'aggregato di elementi di un territorio per proporre una offerta turistica distintiva sostenibile e competitiva. La letteratura internazionale offre molteplici contributi sul turismo a livello sistemico (Laws, 1995, Leiper 1990) lo sforzo è qui prevalentemente di dare "una dimensione collettiva alle strategie e alle aqioni dei singoli attori impegnati nel turismo della destinazione, nella direzione di identificare possibili soggetti, centri decisionali, strumenti, processi” (Tamma, 2002:21).

L'approccio sistemico alle località turistiche ha ricevuto, recentemente, una notevole importanza come evidenziano questi approcci. Questa influenza ha avuto un riconoscimento anche nel testo unico del turismo: la L. 135/2001 sui sistemi turistici locali di cui trattiamo nel quinto paragrafo. Nel prossimo paragrafo, invece, analizziamo quei modelli territoriali di origine industriale che sono stati estesi all'industria turistica (cluster, distretto, milieu) che pongono prevalentemente l'accento sull'analisi di un territorio e del sistema di attori localizzati.

\subsection{MODELLI TERRITORIALI DI ORIGINE ECONOMICO-INDUSTRIALE}

\section{APPLICATI AL TURISMO}

\subsubsection{Il Sistema Territoriale Locale}

Un primo modello territoriale esteso alle attività turistiche è il sistema territoriale locale di Dematteis (2003). Alcuni autori evidenziano come un sistema turistico possa essere definito come un modello di specializzazione produttiva dei sistemi locali territoriali (Giannone, 2002). Il turismo in tal senso rappresenta il fattore di specializzazione specifico. 2003):

Il sistema territoriale è caratterizzato principalmente da quattro fattori (Dematteis,

a. La rete locale di soggetti, che comprende sia la rete di soggetti sia le relazioni che si intrecciano a livello locale tra i vari attori;

b. Il milieu territoriale;

c. Le interazioni tra rete locale, milieu territoriale ed ecosistema;

solo spesso non viene costituita un consorzio di gestione, ma il ruolo del comando risulta diffuso e non accentrato nel sistema locale. Esiste, in altre parole, una sorta di imprenditorialità diffusa a livello locale (Zanni, 1995).

Infine, questo modello ha basi strategico-manageriali al fine di sviluppare vie strategiche con chiari obiettivi e si costituisce, maggiormente, su un'ottica di "progetto" in cui esiste un soggetto, che si erge a rappresentanza degli interessi del luogo. 
d. Le interazioni tra reti locali e sovra-locali.

L'approccio si arrichisce quindi del milien definito come l'ambiente socioeconomico innovativo che è un elemento essenziale del sistema territoriale (Dematteis, 2003). Si integra quindi il ruolo del milieu e dei network interpersonali agli attori, alle relazioni tra questi, alle risorse e ai sistemi locali e globali,. Si configura inoltre una unione tra l'ambiente produttivo e l'ambiente socio-economico. Pensiamo innanzitutto che questi due aspetti siano complementari per la riproducibilità di un modello locale nel tempo. Approfondiamo l'approccio del milieu nel prossimo paragrafo.

\subsubsection{Il Milieu turistico}

Il concetto di Milieu innovateur proviene dalla letteratura di origine francese e svizzera $i$ cui scritti principali furono sviluppati da Aydalot negli anni Ottanta e successivamente tramite gli studi del gruppo GREMI ${ }^{10}$, tra cui Maillat, Crevoisier, Camagni, etc. Alcuni contributi del gruppo GREMI, in particolare, hanno cercato di estendere questo modello territoriale ad altri settori, come ad esempio quello della cultura di Costa (2001) esteso a due quartieri della città di Lisbona.

Uno dei lavori principali sui milieu turistici è quello di Peyrache-Gadeau (2003). L'autrice analizza lo sviluppo di una località di montagna con un modello caratterizzato da un milieu socio-economico a vocazione turistica. Sono descritte due tipi di stazioni sciistiche: le stazioni-territorio di economia turistica e le stazioni-luoghi di produrione turistica. Il primo gruppo si caratterizza per la presenza di un milieu costituito da attori locali spontaneamente aggregati, che si sviluppano al fine di far decollare tutta l'area locale dove le innovazioni sono diffuse e l'organizzazione ha continuità con quella preesistente. Nelle stazioni luoghi di produrioni turistica, invece non esiste un vero e proprio milieu innovateur, gli attori hanno origine esogena e lo sviluppo è meramente turistico e spesso crea una frattura con la storia del luogo. Tramite questa classificazione l'autrice presenta località che sviluppano traiettorie molto diverse negli ultimi anni (Peyrache-Gadeau, 2003) dallo sviluppo per le stazioni-territorio al declino per le stazioni-luoghi.

La teoria del milieu innovateur si focalizza principalmente sul ruolo dell'ambiente socio-economico innovativo e sulla capacità di creare cicli virtuosi di sviluppo. Si rileva soprattutto la maggiore enfasi del legame socio-economico fra comunità produttiva e comunità sociale tipico anche nell'approccio del distrette industriali come entità socioterritoriale.

Alcuni studi della stessa origine si sono sviluppati anche nella letteratura italiana ed estendono l'idea del milieu senza definire il concetto a livello turistico. Si rinvia, per esempio, al lavoro di Bramanti (1997) sul milieu turistico del Garda Bresciano ed al lavoro di Pilotti sull'area sistemica gardesana $(1997)^{11}$.

\footnotetext{
${ }^{10}$ Group europenne de recherche sur le milieu innovateur (European Network on Innovative Environment)

11 Bramanti, in particolare, indica come questa area pur non configurandosi come sistema integrato - le connessioni tra imprenditori risultano ancora deboli come pure i rapporti di cooperazione associazionistica la connotazione turistica dell'area coincide con un'immagine sistemica. Per esempio, la sponda bresciana (da Sirmione a Limone $59 \mathrm{~km}$ ) risulta caratterizzata da una specializzazione nel comparto turistico, con una percentuale occupata nel terziario del 80\% a Sirmione, Salò, Gardone, Riviera e Limone. Questi alcuni aspetti della zona in cui alle attività turistiche lacuali sfruttano i vantaggi di economie di scala, esterne e di agglomerazione. Si avvale, infatti, di un mercato del lavoro con abbondanza di forza-lavoro disponibile, di un mercato finanziario idoneo, di un mercato degli input differenziati ecc.
} 


\subsubsection{Il Cluster Turistico}

Il crescente interesse relativo ai cluster industriali trae la sua origine negli studi di Michael Porter. Porter ha rilevato come in un'economia globalizzata i vantaggi competitivi sono sempre di più legati ad economie locali e radicate in luoghi specifici. In "The competitive advantage of nations" nel 1990, l'autore dimostra come le imprese abbiano la tendenza a localizzarsi in una particolare area ed a formare dei cluster localizzati ${ }^{12}$ e così definisce un cluster "..un gruppo di imprese interconnesse e di istiturioni associate operanti in un particolare campo, territorialmente contigue e collegate da elementi di comunanza e complementarieta”' (Porter 1998:78). ${ }^{13}$

In particolare, la OECD, riassume che: "il concetto di cluster ha il suo focus sui legami e sulle interdipendenze tra gli attori nella catena del valore attraverso la produrione di beni e servizi e tramite l'innovazione..[..]. I clusters risultano spesso networks di imprese, trasversali a più settori, costituiti da imprese specializzate attorno ad uno specifico link o conoscenza nella catena del valore." (OECD, 1999)

Gli studi di Porter si sono riferiti principalmente ai settori manifatturieri non mancano tuttavia riferimenti anche all'industria turistica:

"a host of linkages among cluster members result in a whole greater than the sum of its part. In a typical tourism cluster, for example, the quality of a visitor's experience depends not only on the appeal of the primary attraction but also on the quality and efficiently of complementary businesses such as hotels, restaurants, shopping outlets and transportation facilities. Because members of the cluster are mutually dependent, good performance by one can boost the success of the others" (Porter 1998:77).

Gli studi sui clusters turistici risultano, in ogni modo, abbastanza limitati. Il lavoro principale è quello della Nordin (2003) che sviluppa un approccio porteriano. L'autrice applica in particolare il diamante di Porter estendendo questo concetto all'industria turistica e focalizzandosi sugli aspetti relazionati all'innovazione di una destinazione turistica.

Nordin pone l'attenzione principalmente sulla necessità di sviluppare strategie di collaborazione e sinergie tra gli attori allo scopo di raggiungere un vantaggio competitivo sostenibile di un luogo. Sono proposti alcuni esempi reali di "clustering": Il tourist cluster della Napa Valley, un cluster turistico in Sud Africa ed alcuni esempi in Svezia. L'approccio di clustering, nonostante sia di impatto evidente, tuttavia è concentrato nel creare un network di attori al fine della valorizzazione di un luogo e rimane vago a livello definitorio, sia di imprese, che generale. Note sono le critiche al concetto di cluster di Martin e Sunley di cui troviamo traccia anche nel contributo della Nordin:

"L'approccio di Porter alla competizione ed alla strategia competitiva sono lontane da essere universalmente accettate nelle aree di business economics, industrial organization and management studies dovuto a problemi di essere spesso superficiali, mancano di specificità ed essere difficilmente misurabili" (Martin e Sunley, 2003; opera citata da Nordin, 2003:25) ${ }^{14}$.

Nonostante ciò, l'approccio di cluster porteriano si è diffuso anche nel turismo ed ha assunto una particolare dizione cioè "clustering strategy". La strategia mira appunto a

\footnotetext{
12 Nonostante il concetto di cluster non sia affatto chiaro, utilizzeremo in questo scritto il concetto di cluster porteriano, rimandiamo al contributo "Regional Cluster in Europe" (E.C., 2003) per una rassegna sulla diverse scuola di pensiero sui cluster: Californiana (Scott e Storper), Nordica (Asheim, Amin, Isaksen, Cooke) e porteriano.

13 Il concetto che potremmo estrapolare è quello di una agglomerazione di imprese e si differenzia dal distretto industriale becattiniano per la profonda rete di relazioni sociali e culturali proprie della comunità locale e dalla caratteristica presenza nei distretti italiani delle piccole e medie imprese.

${ }^{14} \mathrm{Si}$ veda inoltre per una survey relativa al concetto di cluster il lavoro di Bergam e Fesser (1999).
} 
sviluppare iniziative allo scopo di coinvolgere e mettere in rete gli attori in un luogo per raggiungere quei tipici vantaggi dei cluster industriali.

In riferimento a quanto detto citiamo per esempio alcuni studi sui clusters del turismo in America (Napa valley, Niagara Falls, etc.) e la cluster initiative (Cluster Consortium, 1999) effettuata in Sud Africa e legata allo sviluppo di un progetto per rilanciare il turismo sudafricano. L'analisi del cluster in Sud Africa ha come scopo principale quello di incrementare le attività di supporto e strettamente relazionate al turismo nazionale. In particolare è effettuata un'analisi delle forze e debolezze dell'industria dei viaggi e del turismo a livello nazionale, applicando il diamante porteriano ${ }^{15}$. Il consorzio, costituito da attori pubblici e privati, mira a migliorare la catena del valore nei suoi elementi verticali ed orizzontali; restano tuttavia valide le critiche proposte nei capoversi precedenti sul risultato di una strategia essenzialmente top-down.

La teoria dei cluster risale direttamente al ruolo delle economie esterne marshalliane come fonte di competitività dei sistemi di impresa (Porter 1998). In particolare un ruolo maggiore è riconosciuto non tanto alla concentrazione, quanto alla struttura dell'industria e all'intensità della rivalità tra imprese. I contributi seguiti a questo paradigma, hanno, posto la propria attenzione sul ruolo della rivalità dell'industria, che spinge le imprese a competere più intensamente ed efficientemente ed a sviluppare innovazioni.

Altri studi di questo tipo utilizzano questo modello territoriale per analizzare il sistema turistico di un luogo e gli attori nell'ottica di proporre un progetto di networking. Si veda per questo Santos (2002) su un cluster in Portogallo, Van Den Berg, Braum, e Van Winden (2001) sul cluster turistico di Amsterdam ed i lavori del Competitivness Insitute relativi ai cluster di Bilbao (1998), San Sebastian (1998) e Lloret de Mar (1998).

\subsubsection{I1 Distretto Turistico}

I lavori sviluppati sui distretti turistici sono un numero esiguo. Uno di questi è il rapporto ACI-CENSIS (2002): "I distretti turistici italiani: l'opportunità di innovare l'offerta", che analizza il territorio italiano e approfondisce: "Un nuovo modello di offerta, che trae origine dalle vocazioni naturali (mare, arte, montagne, ecc.), ma supera la tradizionale "lettura per punti" (i comuni turistici), "linee" (la costiera romagnola o amalfitana) o "sistemi" (V Val Gardena, Val Pusteria, ecc.).

Attraverso alcuni indici ${ }^{16}$ questo studio rileva 299 "distretti turistici" italiani, (96 marini, 37 artistico-culturali, 137 montani e 29 integrati), per un totale di 2.841 comuni con 22,4 milioni di abitanti, dove operano 16.600 ristoranti e 24.300 alberghi e che hanno fatto registrare complessivamente oltre 200 milioni di visitatori. L'analisi suddivide i distretti turistici in distretti marini, artistico-culturali, montani ed integrati.

\footnotetext{
${ }^{15}$ L'analisi evidenzia come il sistema distributivo e la struttura ricettiva necessitino di uno sviluppo e di una migliore integrazione. L'obiettivo principale nel futuro sarà, appunto, quello di aumentare gli accordi e le sinergie all'interno del cluster nazionale.

${ }^{16}$ Sette gli elementi distintivi di un distretto: grado di terziarizzazione (indicatore sul quale è stato costruito il rating): il quadro di evoluzione dell'offerta di servizi, in relazione alla vocazioni primarie ed alle opportunità di divertimento, shopping, sport; qualità ristorazione (concentrazione punti di ristorazione di alta qualità); qualità ospitalità (concentrazione di alberghi segnalati dalle guide più importanti); segmentazione ristorazione (grado di diversificazione dell'offerta gastronomica; segmentazione ospitalità (grado di diversificazione dell'offerta alberghiera: numero di stelle); antinomia omogeneità contro integrazione (intreccio delle vocazioni dei diversi comuni che costituiscono il singolo distretto), "plus enogastronomico".
} 
Il documento evidenzia i luoghi turistici del territorio italiano in base alla presenza di molteplici attività ${ }^{17}$. Tale concetto non coincide con il distretto tradizionale e da questo punto di vista è un'analisi basata maggiormente sul concetto di cluster porteriano dove mancano i legami tra imprese e comunità sociale.

In alternativa, altri studi utilizzano il termine "distretto" come riferimento territoriale di una località focalizzando l'attenzione su altri aspetti. Nel lavoro sul distretto turistico di Livigno $^{18}$ (Molteni, Sainaghi, 1997) lo studio si basa sull'analisi del ruolo di un meta-manager del sistema locale di Livigno configurata nell'Azienda di Promozione Turistica Municipale (APTM) ${ }^{19}$.

Il contributo di Santarelli (1995) prende in esame il distretto turistico nel medio Adriatico, considerando le province di Forlì, Ferrara, Ancona, Ascoli Piceno e Macerata. In questo lavoro studio approfondito, che utilizza come riferimento la teoria distrettuale, il sistema locale analizzato è caratterizzato da basse barriere all'entrata e da livelli trascurabili di costi non recuperabili di uscita, che determinano un intenso processo di natalità e mortalità tipico anche per le imprese (industriali) distrettuali ${ }^{20}$. Nel distretto Emiliano si ha una forte divisione e specializzazione del lavoro tra imprese; le imprese alberghiere sono separate da quelle che offrono servizi ricreativi, da quelle che offrono trasporti, etc. (Santarelli, 1995:350). L'autore definisce il sistema locale turistico:

"La riviera romagnola emerge, in effetti, come un sistema locale specializzato nell'offerta di prodotti turistici e da questo punto di vista, appare del tutto simile ai distretti industriali a vocazione manifatturiera largamente diffusi nelle regioni centrali e nord-orientali del paese. " 21

Uno studio rilevante sul distretto turistico è quello di Antonioli-Corigliano (1999), che analizza un distretto turistico-enogastronomico, approfondendo il ruolo di una strada del vino come centro servizi. L'autrice descrive il distretto turistico riprendendo la definizione becattiniana (2000:54) come: "[...] uno stabile e ragionevole ispessimento di relarioni tra le imprese nel tempo" (Corigliano, 1999:145).

Le imprese sono considerate fortemente radicate nel territorio, la cui identificazione non può essere solo rilevata tramite parametri geografici, ma si deve riferire necessariamente anche a parametri socio economici ${ }^{22}$. Inoltre, ogni impresa è come un ingranaggio del motore che forma il sistema locale. La presenza di simili relazioni tra gli agenti in una piccola area favorisce quella che Marshall chiamava "industrial atmosphere", ripresa poi da Becattini (2000), che l'autrice chiama "atmosfera eno-turistica" per un

\footnotetext{
17 Si rimanda al capitolo due ed allo stato dell'arte dei SLT per una disamina della metodologia utilizzata in questo studio.

18 Stazione sciistica situata a 1.800 metri ai confini dell'Italia con la Svizzera

$19 \mathrm{Nel}$ sistema locale vari interventi sono stai svolti dall'APTM nel corso degli anni dalla risoluzione ai problemi del traffico, al rinnovamento degli impianti di risalita alla realizzazione di un percorso di mountan bike, alla ristrutturazione degli immobili in maniera tipica del luogo, promozione di iniziative di vario tipo, modifica orario apertura dei negozi. Inoltre, nel 1990 l'azienda ha definito un codice di autoregolamentazione per classificare l'offerta extralberghiera, appartamenti e residence, in base alle sue caratteristiche qualiquantitative, numero di vani, posti letto, servizi erogati. Anche se l'APTM ha svolto un ruolo maggiormente di promozione del sistema locale turistico, risultano alcuni casi di concertazione tipica di un sistema produttivo locale, normale e straordinaria. Si veda Dei Ottati (2001) per approfondimenti di questa classificazione.

${ }^{20}$ Si veda Lazzeretti e Storai (1999) per la natalità e la mortalità del distretto industriale di Prato.

21 Il distretto si caratterizza da alcuni fattori: 1) Intensità del processo di formazione di nuove imprese, incubators of entrepreneuship; 2) Processi cumulativi di apprendimento basati su un'offerta di lavoro professionalmente caratterizzata; 3) Economie esterne che ridimensionano il ruolo delle barriere all'entrata e affievoliscono i problemi della piccola dimensione di impresa.

22 Si pensi, invece, alla metodologia di individuazione nel rapporto ACI-Censis.
} 
distretto turistico come un: "[...] particolare stato in cui le informazioni, la cultura, le innovazioni, le abilità sono trasmesse ed apprese in una maniera semi-inconscia e scambiate grazie all'azione indiretta delle istituzioni sociali (famigli, scuola, imprese, associazioni, e cosi via) e dalla prossimita" (ibidem:150) ${ }^{23}$.

La teoria del distretto risale direttamente alle economie esterne marshalliane come fonti di competitività dell'area locale. Un'interessante riferimento a queste fonti di competitività in relazione ad un distretto turistico è proposta anche in questo contributo. Corigliano (1999) suddivide le economie di scala in economie di promozione e commercializzazione a livello di impresa e a livello di sistema locale ${ }^{24}$; ed in economie di agglomerazione, esterne all'impresa ma interne al distretto turistico. Tralasciamo tuttavia questo tema relativo alle fonti di competitività dei sistemi di impresa che tratteremo specificatamente nel terzo capitolo.

I modelli dei distretti turistici, presentati fino ad ora, analizzano una località nella sua interezza come distretto turistico. Gli studi internazionali specialistici sul turismo si concentrano, invece, su limitate aree urbane ${ }^{25}$ e su quartieri come distretti turistici. Ad esempio, Pearce (1998) analizza la città di Parigi ed esamina le diverse caratteristiche strutturali dei quartieri turistici nella capitale francese. Il contributo si focalizza principalmente su diversi nuclei cittadini che si configurano come eterogenei distretti turistici: Ile de la Cite', Montmartre e il quartiere dell'Operà. Altri autori analizzano il recreational business district delle cascate del Niagara (Stansfield, Richert, 1970) o il tourism business district (Getz, 1993). Questi studi hanno la caratteristica di analizzare un'area geografica circoscritta e limitata, ad esempio un quartiere urbano, come un distretto turistico. Un altro autore, per esempio Judd, analizza invece il ruolo delle economie di agglomerazione in un distretto turistico urbano:

"Agglomeration economies apply to tourist district not principally because concentration lower costs or increase the efficiency of business transaction, but because a full panopoly of services and business is necessary to make the space maximally attractive to consumers of the tourist space" (Judd, 1993:179).

L'autrice rileva come si sviluppano economie di agglomerazione quando la localizzazione di un'unità economica dipende dalla localizzazione di tutte le altre e non perché esistono dei vantaggi economici o di efficienza, ma perché in un'area circoscritta un'offerta completa di servizi è necessaria per massimizzarne l'attrattività. Judd non sviluppa questo tema, ma include nelle sue riflessioni anche attività ricreative per il tempo libero, centri congressi, etc.

Jansen-Verbeke e Ashworth (1990) affermano invece che lo sviluppo del turismo in un luogo dipende più dalla concentrazione che dalla dispersione, dalla combinazione funzionale anziché dalla segregazione e dagli ambienti multifunzionali più che dalla monofunzionalità. Ashworth (1990) analizza queste relazioni distrettuali nelle città storiche di Groningen e Londra considerando l'intera città come un distretto unico.

\footnotetext{
${ }^{23}$ L'impannatore pratese o imprenditore puro potrebbe configurarsi con il ruolo del tour operator che al fine di effettuare le proprie attività, gestisce informazioni sui mercati nazionali e su quelli internazionali e sulla fornitura di servizi turistici.

${ }^{24}$ Economie di specializzazione e ampiezza (o scopo) dovute all'offerta di servizi specializzati nel sistema locale;

${ }^{25}$ Gli studi sul turismo urbano si sono sviluppato recentemente intorno agli anni novanta, in principio questo tema di ricerca era principalmente sviluppato da geografi (Jansen-Verbeke 1986; Pearce, 1981). Nell'ultima decade invece l'attenzione posta al turismo urbano è incredibilmente aumentata. Tra gli studiosi europei principali possiamo citare Cazes e Poitier, Van der Berg, Van der Borg, Van der Meer, Ashworth, Marchesa Gomez.
} 
Si evidenzia, a nostro parere, come gli approccio sistemici per lo studio di una località turistica siano di crescente rilevanza negli ultimi anni. Nel prossimo paragrafo proponiamo un modello il sistema locale turistico al fine di analizzare un sistema di produzione turistica, utilizzando come punto di partenza l'approccio della teoria distrettuale.

\subsection{IL MODELLO DI RIFERIMENTO: IL SISTEMA LOCALE TURISTICO}

\subsubsection{Il punto di partenza: l'approccio distrettuale}

L'obiettivo di questo paragrafo è focalizzare il legame tra l'approccio distrettuale ed il sistema locale turistico, come modello di sviluppo locale in grado di produrre ricchezza e occupazione. Ne "Il caleidoscopio dello sviluppo locale", Becattini, Bellandi, Dei Ottati e Sforzi (2001) approfondiscono il legame del distretto industriale e la sua teoria con i molteplici sentieri di sviluppo locale italiano. Nell'articolo di Bellandi e Dei Ottati gli autori cercano di "dare un ordine all'espansione dell'approccio distrettuale" con gli altri modelli di sviluppo locale.

Analizzando l'articolo, si percepiscono due assi di caratteristiche fondamentali del distretto industriale: l'organizzazione produttiva, il sistema di imprese localizzato e specializzato - che effettua un'integrazione flessibile della divisione del lavoro - e l'ambiente socio-istituzionale locale. E' proprio l'interazione di questi due assi che rende il distretto industriale un modello auto-riproduttivo ${ }^{26}$ di sviluppo locale.

La considerazione di fondo è che il distretto industriale non risulta il solo modello di sviluppo locale, ma risulta una importante chiave di lettura. Altri modelli possono essere il polo industriale, la città dinamica, il milieu innovateur ${ }^{27}$ ed infine il sistema locale rurale. In altre parole i luoghi di grande e piccola impresa e i loro ibridi, che connessi costruiscono modelli di sviluppo locale distinti e unici: "La città, il sistema rurale, dove convivono grandi e piccole imprese, il polo industriale di grande impresa e $i$ nuclei industriali della piccola impresa marginale o dipendente in cui convivono i caratteri urbani e rurali" (Bellandi e Sforzi, 2001:45).

Il nostro intento è quello di risalire ad un modello di sviluppo locale ibrido: il Sistema Locale Turistico (SLT) dove possono convivere piccole e grandi imprese. La prima fase è di definire un'agglomerazione di imprese specializzate in attività turistiche e verificare la sua capacità di autoriprodursi, generare occupazione e produrre ricchezza, avendo cura di chiarirne la dimensione territoriale e le caratteristiche socioculturali specifiche locali. La nostra analisi si focalizzerà su imprese accomunate da una base di esperienze lavorative e sociali $^{28}$, in realtà locali, legate allo sfruttamento sostenibile di risorse ambientali, artistiche e culturali. Tali processi sono diffusi nelle nostre campagne, coste, e destinazioni turistiche, dove le imprese e le comunità locali mirano alla valorizzazione del patrimonio artistico, culturale ed ambientale.

Infine, seguendo l'approccio di Bellandi e Sforzi (2001:48 ss.) i diversi tipi di impresa che si possono trovare in un sistema locale turistico sono molteplici. Da un lato ci sono imprese legate alla valorizzazione delle risorse turistiche proprie dell'area, dall'altro, imprese di tipo non caratterizzanti: artigiane ad alto contenuto storico culturale, legate alla domanda locale di beni e servizi o all'offerta locale di lavoro e infrastrutture, ed imprese locali non radicate. Le imprese (agricole, industriali, commerciali, turistiche) si relazionano con le tradizioni di vita

${ }^{26}$ Becattini (2000) cita anche il modello autopoietico di Maturana e Varela.

27 Si rimanda a Maillat (1998) per le caratteristiche e i fondamenti del Milieu Innovateur.

28 Si pensi ai sistemi locali del lavoro di Sforzi (1997a). 
e lavoro e con l'insieme di risorse artistiche, culturali e ambientali. Questi legami in essere ed in evoluzione, sono la base per la costruzione di un vantaggio competitivo dell'area locale ${ }^{29}$.

\subsubsection{Alcuni concetti di base: il PACA ed il cluster di attori}

\section{Il Patrimonio Artistico Ambientale e Culturale}

La presenza che sta alla base di un sistema locale turistico è il patrimonio di risorse da valorizzare e promuovere. L'emersione dei distretti industriali è stata spesso accompagnata dalla valorizzazione di risorse locali da parte di imprese leader della comunità locale (Trigilia, 1986).

Da questo punto di vista vogliamo porre l'attenzione sulla presenza di patrimonio artistico culturale ed ambientale (PACA), introdotto da Lazzeretti (1997:699). Il PACA si riferisce non solo alla parte materiale delle risorse di un luogo, monumenti e opere d'arte, ma costituisce il set di risorse materiali ed immateriali riconducibili a tre categorie:

- artistico: monumenti, complessi architettonici, opere d'arte, edifici, ecc.;

- culturale: attività e mestieri tipici, senso di appartenenza, atmosfera dei quartieri tipici della città, usi e costumi, folklore, spettacoli e manifestazioni, ecc.;

- ambientale: bellezze naturali, paesaggio, giardini, parchi, piazze, flora e fauna caratteristica.

Si configurano così città, coste, vallate, e destinazioni turistiche che possiedono diverse dotazioni del fattore $P A C A$. In base a questo fattore è possibile mappare un luogo, una nazione o una regione ${ }^{30}$ in base alla sua dotazione.

Questo patrimonio può inoltre configurarsi come un possibile volano di sviluppo; infatti, può acquisire un ruolo economico se avviene il passaggio da risorse da conservare a risorse da valorizzare (Lazzeretti e Cinti, 2001). A questa condizione, il PACA può trasformarsi in un fattore endogeno di sviluppo dando luogo a motori locali alternativi all'industrializzazione $\mathrm{e}^{31}$.

\section{Il cluster di attori}

Negli ultimi anni, un importante dibattito è emerso in relazione all'estensione dei modelli territoriali di origine economico-industriale (distretto, cluster) ad altre industrie, ad esempio come per i distretti culturali sia in Italia (Preite, 1998, Valentino, 2001, Santagata,

\footnotetext{
${ }^{29}$ I legami forti fondamentali sono i legami tra società locale e le imprese che valorizzano il patrimonio di risorse e tradizioni, quelli deboli sono nei mercati locali di fase e nelle istituzioni focalizzate su particolari attività (per esempio l'offerta alberghiera, etc.).

${ }^{30}$ Si rinvia al lavoro di Bacci (2002) per la georeferenzazione del patrimonio artistico e culturale della regione toscana.

31 Oltre alla necessità della conservazione i beni considerati, non possono essere valutati solo come risorse, $\mathrm{ma}$, a nostro parere, devono essere valutati anche in termini di vincoli. Il loro sfruttamento non può essere illimitato, si pensi ad un area protetta che viene eccessivamente sfruttata o alle troppe città d'arte in cui l'eccessivo sfruttamento in termini turistici può porsi in conflitto con la comunità locale (Mossetto, 1992). In conclusione, non approfondiamo il tema della sostenibilità, per ovvi motivi di spazio e tempo, ma vogliamo puntualizzare che il patrimonio artistico, culturale ed ambientale (PACA) può assumere il ruolo di un autonomo volano di sviluppo sostenibile se adeguatamente mantenuto e valorizzato.
} 
2000, Lazzeretti, 2003; 2004) che all'estero (Garreau, 1998, Scott, 1996, Frost Kumpf, 1998). Estendendo in particolare l'approccio di Lazzeretti (2003) (per il distretto culturale), il sistema locale turistico rappresenta un sistema caratterizzato dalla presenza nella destinazione turistica di "un network di attori economici, non economici ed istituzionali, interconnessi, di piccola e grande dimensione che creano ricchezza e occupazione tramite il legame con le risorse" (Lazzeretti, 2004:22) del luogo turistico ${ }^{32}$. Il cluster è quindi composto da imprese alberghiere, di ristorazione, agenzie di viaggio, turistiche in senso ampio ${ }^{33}$ ed da istituzioni e associazioni varie. Il cluster si configura come un elemento fondamentale per la valorizzazione del patrimonio artistico, culturale ed ambientale e costituisce una delle caratteristiche essenziali di un distretto industriale, ma anche culturale o turistico.

Attraverso questo approccio (Lazzeretti, 2004:26 e ss.) è importante verificare la presenza di un cluster di attori economici, non economici ed istituzionali, interconnessi, di piccola e grande dimensione che creano ricchezza e occupazione tramite il legame con le risorse del luogo turistico.

\subsubsection{Il Sistema Locale Turistico}

In sintesi è essenziale verificare a) la presenza in loco di un patrimonio artistico, culturale ed ambientale; b) di un cluster di attori in grado di produrre ricchezza e occupazione dalle risorse locali, nell'ambito dei servizi turistici (alberghi, agenzie di viaggio, ristoranti, impianti di risalita, ecc.). Queste sono le basi per la configurazione di un Sistema Locale Turistico (SLT).

Le relazioni tra i due blocchi sopraesposti, $a$ e $b$ e la comunità locale, le istituzioni, le tradizioni, insieme ai vari aspetti socio-economici sono il punto di partenza per verificare l'esistenza di gradi diversi di distrettualizzazione turistica come un testi di diversità/uguaglianza con un "tipico distretto turistico Marshalliano"34.

Sono proprio questi due elementi: la popolazione di imprese (il cluster) e la comunità sociale che rendono il SLT un modello auto-riproduttivo di sviluppo locale. Il SLT è in prima approssimazione un gruppo di imprese localizzate, prevalentemente di piccola e media dimensione che genera ricchezza e occupazione attraverso la valorizzazione delle risorse locali (il $P A C A$ ). Nella figura sottostante, mostriamo come un cluster di attori economici (PMI), non economici ed istituzionali sia la condizione sine qua non per la costituzione di un SLT.

\footnotetext{
32 In particolare Lazzeretti (2001) va in cerca di HCCluster (High Cultural Cluster) nelle città d'arte in Italia.

33 Consideriamo come "Attività connesse al turismo", esclusivamente a titolo di esempio in questa primo capitolo, le attività elencate in appendice E di "ATECO 91 - Classificazione delle attività economiche", ISTAT 1991: Alberghi, Campeggi ed altri alloggi per brevi soggiorni, Ristoranti, Bar, Mense e forniture di pasti preparati, Trasporti ferroviari, Altri trasporti terrestri (escluso 60.25 - Merci su strada), Trasporti aerei di linea, Attività delle agenzie di viaggio e degli operatori turistici, Attività delle ag. di viaggio e turismo (compresi i tour operator), Attività di guide e accompagnatori turistici, Noleggio di autovetture, Noleggio di altri mezzi di trasporto.

${ }^{34} \mathrm{Si}$ fa riferimento esplicitamente ai processi di distrettualizzazione esposti da Becattini (2001) in base a diversi gradi di trasformazioni di un luogo in un distretto industriale di tipo marshalliano. Lo studio dei processi di distrettualizzazione è un analisi dinamica del distretto focalizzato non sul distretto in sé, ma su processi di analisi. Partendo dalle riflessione di Becattini sul distretto agricolo (Becattini, 200:197), per esempio, Lazzeretti (2003:641) analizza un distretto culturale con il supporto di una completa analisi ex-post sviluppata su un distretto maturo di tipo Marshalliano. L'autrice cerca di individuare diversi gradi di distrettualizzazione culturale nei diversi sistemi locali culturali che sono più o meno sviluppati in confronto all'ideltipo di "tipico distretto culturale Marshalliano".
} 
Figura 1.1: Il Sistema Locale Turistico (SLT): il cluster di attori e la comunità sociale.

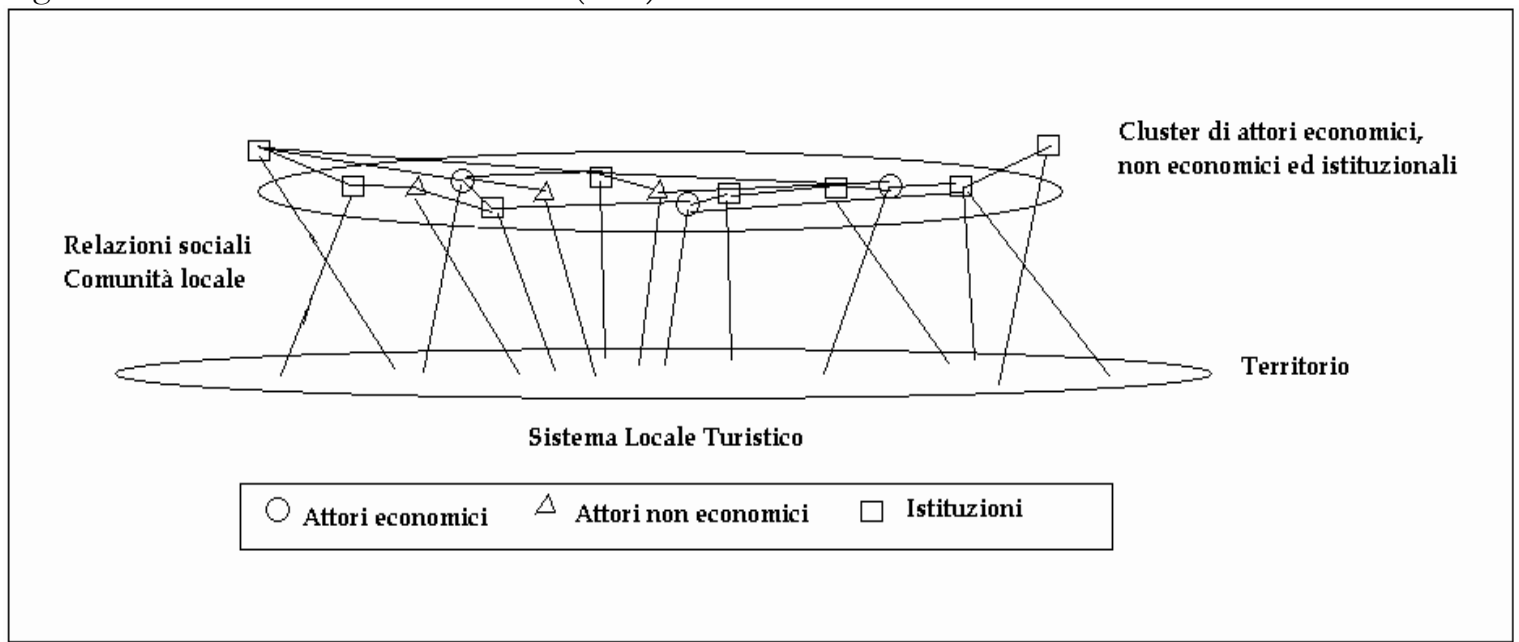

Fonte: nostra elaborazione;

Le relazioni sociali con la comunità locale, costituiscono l'altra condizione necessaria per la riproduzione del modello di sviluppo locale nel tempo. Nella figura, un cluster di attori interconnessi e localizzati sviluppa relazioni con la società locale tramite le linee verticali. L'ispessimento delle relazioni sviluppa in un ambiente circoscritto effetti riproduttivi e instaura l'bumus necessario per lo sviluppo economico del luogo.

Il SLT si configura quindi come un "tessuto localizzato di relazioni socio-economiche, fra $i$ diversi membri della società locale, tale da favorire la formazione, la diffusione e il mantenimento di un sistema di valori, di conoscenze produttive, di comportamenti tipici e d'istituzioni attraverso i quali la società locale interagisce con l'organizzazione produttiva" (Becattini e Sforzi, 2002:21) ${ }^{35}$, caratterizzata prevalentemente da attività turistiche.

Il SLT può essere, quindi, descritto, in prima approssimazione estendendo l'approccio distrettuale (Bellandi, 2003), come caratterizzato principalmente da una serie di elementi:

i. Il SLT è prima di tutto un luogo in cui la comunità sociale ed economica convive giornalmente $^{36}$;

ii. Le attività produttrici di reddito sono caratterizzate da un'industria stabile e principale quella turistica e da altre attività connesse denominate ausiliari;

iii. L'attività produttiva principale è localizzata nel sistema locale, cioè svolta da persone ivi residenti e una parte importante della produzione è effettuata al suo interno. Il patrimonio artistico culturale ed ambientale locale influenza fortemente questa componente. Non c'è la possibilità di lasciare l'area locale dove sono le risorse;

iv. Un numero elevato di attività produttive distinte e specializzate è localizzato nel SLT. Se immaginiamo la filiera dell'offerta turistica, questa è rappresentata più o meno interamente nel SLT ${ }^{37}$.

\footnotetext{
35 Parafrasando le parole di Becattini si definisce un SLT come un Sistema locale del lavoro specializzato nell'industria turistica, come vedremo nel secondo capitolo.

${ }^{36}$ Si pensi per esempio al ruolo dei sistemi locali del lavoro nell'identificazione dei distretti industriali e al ruolo di unità di analisi socio-economiche.

37 Riprenderemo questo punto nel capitolo 3, essendo consapevoli che sia necessario approfondire la filiera dell'offerta di un prodotto turistico.
} 
v. In un SLT le imprese sono prevalentemente di piccola e media dimensione, nel senso che vi è l'assenza di un'impresa dominante ed esiste una popolazione di imprese autonome ${ }^{38}$.

Approfondendo i temi sopra esposti, per quanto riguarda la comunità locale, il fattore caratterizzante di un SLT può essere la presenza nella popolazione di un sistema omogeneo di valori (etica del lavoro, dell'attività, della famiglia, del cambiamento). La delimitazione territoriale e la persistenza nel tempo può favorire la creazioni di vere e proprie comunità locali con valori sociali comuni, si pensi, per esempio, alle comunità montane.

Le imprese, sono specializzate in una o più fasi del processo di fornitura di servizi turistici ed ogni impresa è parte dell'ingranaggio che muove l'area locale complessiva in una multisettorialità imprenditoriale. All'interno del SLT le imprese sono interprenetrate e possono usufruire dei vantaggi legati alle economie di scala e di varietà. I due processi di concorrenza e competizione interagiscono all'interno del distretto, da un lato vi è un'intensa concorrenza, in cui ognuno cerca di fornire un prodotto o servizio migliore, dall'altro i legami di cooperazione, la interconnessione delle imprese e le relazioni sociali dell'area locale consentono al SLT di mantenere un processo di cooperazione tra imprese che permette "..di poter rischiare e sbagliare" (Dei Ottati, 1995).

Nello sviluppo di un SLT verso processi di distrettualizzazione turistica ${ }^{39}$ il distretto turistico può essere interpretato come una tipologia di sistema locale turistico in cui l'ispessimento delle relazioni economiche e sociali è particolarmente forte, in cui esistono tutti gli elementi tipici della comunità sociale relativi alla fiducia ed al capitale sociale.

\subsubsection{La dimensione multiscala territoriale del SLT}

In questo paragrafo, ci interroghiamo su come individuare i confini di un sistema locale turistico e su come si diffondono gli effetti economici nel territorio. Pearce (2001) riflettendo sulla dimensione spaziale del turismo di un luogo, ed in particolare di una città, propone un framework teorico di analisi per gli effetti economici e non economici delle destinazioni turistiche, dove la dimensione spaziale va dal locale al globale. Esiste una dimensione spaziale a livello di quartiere (o district), urbano, regionale, nazionale ed internazionale. Il turismo è profondamente collegato con i confini del luogo dove è stabilita e programmata la gestione e le politiche. Questo paradigma interpretativo diventa importante anche nelle riflessioni di questo lavoro.

\footnotetext{
${ }^{38}$ È naturalmente possibile che vi siano alcune imprese leader sulle quali è centrata la produzione per squadre. 39 In Lazzeretti (2003) si elencano i processi di distrettualizzazione culturale dei distretti culturali nelle città d'arte italiane ed in particolare a Firenze.
} 
Figura 1.2: Un Framework integrativo multiscala per il turismo;

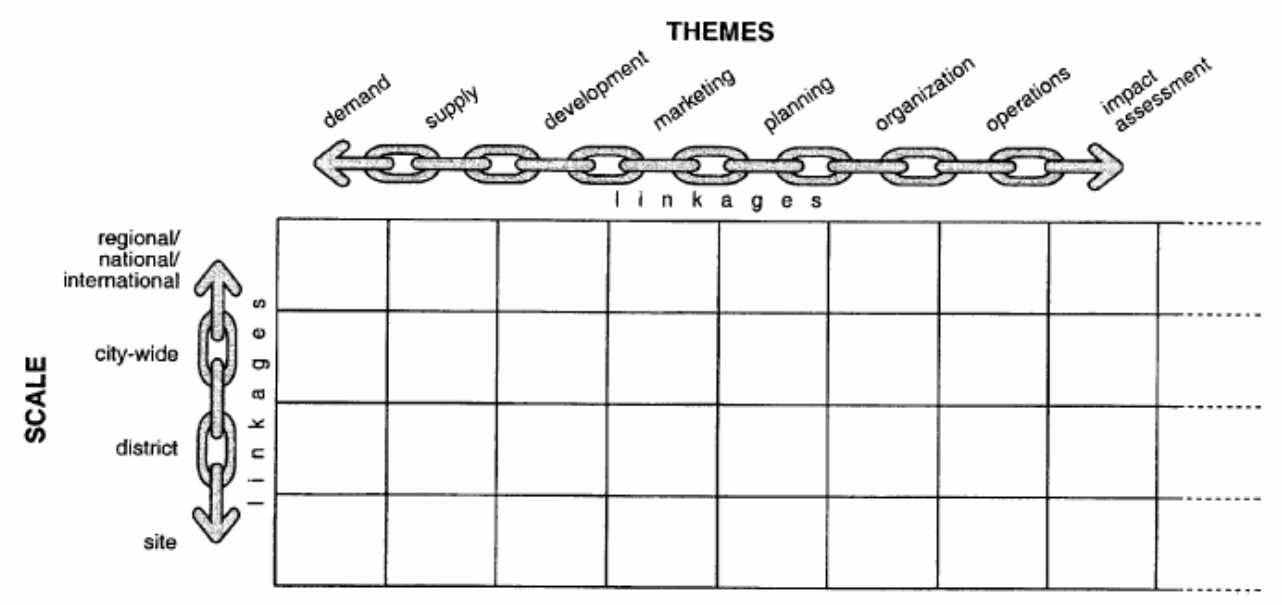

Fonte: Pearce, 2001:935;

Riflettendo sulla figura 1.2 , ci si chiede prima di tutto come definire i confini territoriali di un SLT ed i suoi effetti nell'area locale dato che si configurano SLT come piccole medie-grandi città (destinazioni estive, invernali, città d'arte), aree provinciali, regionali.

Pearce, risolve questo problema, introducendo nel suo studio le reti di destinazioni (gateways) ed analizzando il ruolo nazionale e sovralocale dei gateways. Innanzitutto le destinazioni turistiche svolgono un ruolo di hubs (nodi) di trasporto con un'enfasi sulla loro funzione all'interno di un più vasto network territoriale, ma anche funzioni di altro tipo (informazioni, industriali, trasporto).

L'autore definisce gli bubs come "special nodes that are part of a network, located in such a way as to facilitate connectivity between interacting places". (Pearce, 2001:937).

Il ruolo degli bubs non può comunque essere limitato alla funzione dei trasporti, ma deve comprendere tutti i patterns di viaggi e le modalità con cui questi sviluppano i più diretti ed indiretti legami tra le città di un'area locale ${ }^{40}$.

Per esempio, Lopez-Palomeque (1995) descrive come un'offerta eccessiva di alloggiamento in Barcellona dopo i giochi olimpici del 1992, sviluppò una cooperazione a rete all'interno dell'area metropolitana e tra le coste della Catalogna (Costa Brava e Costa D'oro).

Questo network di destinazioni si può sviluppare quindi sia a livello di offerta che a livello di domanda. In altre parole, sia a livello di interazione e cooperazione a livello della struttura dell'offerta sia di conseguenza (ma non necessariamente) a livello dei visitatori e quindi di itinerari tra destinazioni. Si pensi per esempio alle strade del vino o agli itinerari storici-artistici. Può esistere quindi una rete territoriale di destinazioni turistiche che si sviluppano, cooperano e sono in competizione.

Questo problema del confine dell'unità di analisi e dell'importanza dei confini degli effetti economi e non economici del framework teorico di Pearce è risolto dal paradigma delle reti di città. In altre parole, la definizione dei confini dell'unità territoriale di analisi

\footnotetext{
40 Per esempio, Pearce (2001) descrive come il Trip Index: il numero di notti spese in un luogo in base alla
} durata totale del viaggio al fine di esaminare il ruolo e i network di città turistiche. 
deve essere ponderata profondamente; ci si chiede quindi come definire i confini territoriali di un SLT e dei suoi effetti nell'area, configurando così sistemi autonomi e reti di sistemi.

I principali riferimenti alla teoria delle reti di unità territoriali, in particolare città, sono stati sviluppati da Pred (1977), Dematteis (1989) e Camagni e Salone (1993) e per le reti di città policentriche da Trullen e Boix $(2002,2004)$. Ci è di aiuto anche la teoria degli spillovers spaziali ${ }^{41}$ introdotta nelle analisi economiche regionali di Anselin (1992, 1998, 2001) e nelle rassegne di Glaeser et al. (1992) e Rosenthal e Strange (2002).

Approfondiamo gli aspetti relativi alla definizione dei confini del sistema locale nel quinto capitolo, proponendo una proxi empirica per la misurazione degli effetti "turistici" nelle aree limitrofe ad un SLT. Nel capitolo seguente identificheremo nel territorio italiano i SLT in base agli attori ed alla struttura dell'offerta. In particolare, ci focalizzeremo su quei luoghi dove sono concentrati un elevato numero di attori economici e non economici che valorizzano le risorse locali ed in cui la comunità sociale ed industriale-turistica coincidono. Nel prossimo paragrafo confronteremo questo approccio con quello introdotto con la legge 135/2001.

\subsection{STL E LA LEGGE $135 / 2001$}

In questo paragrafo evidenziamo le differenze tra il framework appena proposto del SLT e un recente approccio sui sistemi turistici introdotto con la legge numero 135 del 2001. La L. 135/2001 è la legge di riforma nazionale del turismo che, oltre a trattare molteplici aspetti legati a questa attività, nell'articolo 5 introduce il concetto di sistema turistico locale (STL) come:

" $i$ contesti turistici omogenei o integrati, comprendenti ambiti territoriali appartenenti anche a regioni diverse, caratterizzati dall'offerta integrata di beni culturali, ambientali e di attrazioni turistiche, compresi $i$ prodotti tipici dell'agricoltura e dell'artigianato locale, o dalla presenza diffusa di imprese turistiche singole o associate" (L. 135/2001, art. 5)

In sintesi si propone un sistema pubblico/privato che promuove, tramite un'offerta integrata, le risorse turistiche, i beni culturali e ambientali, gli eventi etnici e artistici, i prodotti tipici della ristorazione, localizzati in un ambito territoriale facente capo a uno o più comuni con l'eventuale partecipazione di enti territoriali quali Province, Camere di Commercio e Regioni.

L'obiettivo principale di questa legge è stato quello di innovare l'offerta turistica italiana, che non poteva ulteriormente basarsi solo sulle classiche destinazioni turistiche. A tal fine la legge 135/2001 mirava ad innescare un processo di cooperazione a "far sistema" tra gli attori nell'ottica di costituire un'organizzazione turistica integrata per lo sviluppo di un'area.

Dopo tre anni dall'entrata in vigore della 135 , ci sono ancora varie interpretazioni su cosa siano i sistemi locali turistici introdotti con la legge, e mentre alcune Regioni hanno

\footnotetext{
${ }^{41}$ La parola spillover (spaziale) presuppone un certo topo di "diffusione" della conoscenza a livello spaziale: "la conoscenza è spesso scambiata senza essere venduta o comprata, la parola spillovers è in questo senso importante" (Rosenthal e Stange, 2002:30). L'idea principale è che "la prossimità geografica faciliti la trasmissione di idee e la diffusione di conoscenza. [...] attraverso l'imitazione, lo spionaggio, e lo scambio di manodopera tra le imprese, le idee sono velocemente sviluppate e diffuse tra le imprese vicine" (Glaeser et al., 1992:1127).
} 
deciso di applicarla, altre non hanno ancora effettuato modifiche alla legislazione turistica regionale né prevedono di effettuarle in futuro.

A fine del 2003, in base ad un Rapporto sul Turismo effettuato da Il Sole 24 ore (tabella 1.1), le uniche Regioni che avevano applicato la legge nazionale erano le Marche ${ }^{42}$ e l'Umbria. Probabili applicazioni erano ancora al vaglio in Liguria, Lombardia, Lazio, Molise, Campania, Calabria, Sicilia e Abruzzo e attualmente poche di queste regioni hanno recepito la $135 / 2001^{41 b}$. Inoltre i progetti risultavano proposti esclusivamente dall'alto e ben pochi quelli effettuati a livello spontaneo bottom-up ${ }^{43}$. Continuano ad esistere quindi le molteplici forme di promozione e paternariato territoriale presentate durante gli anni come la APT, le IAT e i Progetti di Integrazione Territoriale ${ }^{44}$. Si pensi ad esempio che la Regione Toscana non ha recepito la 135 ed ha mantenuto le APT o ad un PIT, in Sicilia, denominato "Dal Turismo tradizionale ad un Sistema Turistico Locale Integrato".

Tuttora esiste uno scarso riconoscimento della legge ed è aspramente criticata dagli operatori (Becheri, 2003). Inoltre, se da un lato la legge ha stimolato spesso gli attori a costituire un consorzio di mera promozione, lasciando ad ogni operatore le possibilità di agire - delega che in realtà spesso non è recepita a livello locale - d'altro lato rischia di scardinare strategie di marketing sovra-locali con l'obbiettivo di promuovere un prodottoluogo nel mercato (Giannone, 2002).

In sintesi, mentre prima si individuava un territorio e poi si passava alla pianificazione delle strategie di marketing, ora, si individuano prima gli ambiti territoriali di riferimento e dopo - nell'eventuale presenza di un network di attori pubblici e privati - si rende possibile lo sviluppo di un consorzio di gestione e promozione (Di Meo, 2002), che spesso però è lasciato alle iniziative dei singoli.

\footnotetext{
42 In particolare, le Marche hanno nel territorio, 10 SLT. È la prima Regione italiana (d.r.g. 578/2002) ha applicare la 135/2001. Lo schema di applicazione si sviluppa top-down tramite dei parametri di riferimento (significatività dell'area territoriale coinvolta, dimensione territoriale, tipologia del prodotto, omogeneità, integrazione, presenza di più tipologie di offerta (1500 letti), presenza di una rete privata pubblica, dimensione flussi turistici).

${ }^{43}$ Unico esempio un STL costituito tra 15 comuni in Sicilia (Terme di Salinuntino, Mercury, 2001) nella zona tra Sciacca e Mazzara del Vallo.

${ }^{4}$ Si ricorda che i Progetti integrati territoriali sono progetti che possono avere anche una specializzazione turistica e che tramite l'utilizzo di Fondi Strutturali Europei (FSE) i comuni rientranti nell'obbiettivo 2 possono sviluppare dei consorzi di promozione e gestione di un luogo.
} 
Tabella: 1.1: Stato attuale del riconoscimento dei STL in base alla 135/2001.

\begin{tabular}{|c|c|c|}
\hline Regione & Legge regionale & Processo in corso \\
\hline Valle d'Aosta & L.R: $6 / 2001$ & $\begin{array}{l}\text { Istituti gli IAT in sostituzione delle APT. L'organizzazione attuale non subirà } \\
\text { modifiche in base alla } 135 / 2001\end{array}$ \\
\hline Piemonte & L.R. 5/1996 & $\begin{array}{l}\text { L'agenzia regionale e le ATL sono i referenti della promozione e dell'organizzazione } \\
\text { turistica e prevedono già la partecipazione pubblica privata }\end{array}$ \\
\hline Liguria & L.R. $15 / 1998$ & $\begin{array}{l}\mathrm{E} \text { in discussione un DDL di riorganizzazione turistica regionale. } \grave{\mathrm{E}} \text { previsto il } \\
\text { riconoscimento dei STL tra pubblico e privato }\end{array}$ \\
\hline Lombardia & L.R. 28/1986 & $\begin{array}{l}\mathrm{E} \text { in elaborazione il PDL do organizzazione turistica regionale. Entro fine anno } \\
\text { dovrebbe nascere il primo STL pilota della regione. }\end{array}$ \\
\hline Trentino & L.P. $8 / 2002$ & $\begin{array}{l}\text { È stata privatizzata la promozione turistica regionale (Trentino Spa). Sono in fase di } \\
\text { privatizzazione le APT territoriali, in cui è previsto il ruolo del privato. }\end{array}$ \\
\hline Alto Adige & L.P. 3/1992 & $\begin{array}{l}\text { La legge ha istituito } i \text { consorzi turistici, che vengono considerati come STL. Non si } \\
\text { prevedono modifiche in base alla } 135 / 2001 \text {. }\end{array}$ \\
\hline Veneto & L.Q. 33/2002 & $\begin{array}{l}\text { Le precedenti APT sono state organizzate in Sistemi Turistici. Non si prevedono } \\
\text { modifiche in base alla } 135 / 2001 \text {. }\end{array}$ \\
\hline $\begin{array}{ll}\text { Friuli } & \text { Venezia } \\
\text { Giulia } & \end{array}$ & L.R. $2 / 2002$ & $\begin{array}{l}\text { La legge ha istituito le Società d'area, ancora in fase di organizzazione. Queste } \\
\text { rappresentano i sistemi turistici della Regione. }\end{array}$ \\
\hline Emilia Romagna & L.R: $7 / 1998$ & $\begin{array}{l}\text { Nessuna modifica alle unioni di prodotto. Sono allo studio strumenti per trasferire } \\
\text { più mezzi alle Province nello "spirito" della 135/2001. }\end{array}$ \\
\hline Toscana & L.R. $42 / 2000$ & $\begin{array}{l}\text { L'attuale normativa, che prevede una agenzia di promozione regionale e } 15 \text { APT, } \\
\text { non subirà modifiche in base alla } 1345 / 2001 \text {. }\end{array}$ \\
\hline Marche & Del 578/2002 & $\begin{array}{l}\text { La Regione ha riconosciuto } 10 \text { SLT come strumenti di integrazione tra pubblico e } \\
\text { privato. }\end{array}$ \\
\hline Lazio & L.R. 9/1996 & $\begin{array}{l}\mathrm{E} \text { in elaborazione la nuova legge quadro regionale, che dovrebbe prevedere il } \\
\text { riconoscimento dei STL. }\end{array}$ \\
\hline Umbria & L.R. $6 / 2002$ & $\begin{array}{l}\text { La legge ha definito le modalità e le procedure per il riconoscimento dei STL. In } \\
\text { questi giorni la Regione sta procedendo al riconoscimento dei primi STL. }\end{array}$ \\
\hline Abruzzo & L.R. 54/1997 & $\begin{array}{l}\text { Si discute sulle possibilità di creare } 4 \text { STL a livello regionale (aare, montagna, } \\
\text { affari/lavoro, cultura/enogastronomia nella logica territoriale. }\end{array}$ \\
\hline Basilicata & L.R. 34/1996 & $\begin{array}{l}\text { Le aree-prodotto individuate nel piano turistico regionale sono equiparate ai STL. E } \\
\text { in fase di revisione la legge quadro regionale. }\end{array}$ \\
\hline Molise & L.R. 13/1978 & $\begin{array}{l}\text { Presentato il PDL di riordino dell'organizzazione turistica che prevede la creazione } \\
\text { di società di promozione turistica sulla base dei STL. }\end{array}$ \\
\hline Calabria & L.R. $13 / 1985$ & $\begin{array}{l}\text { Sono in fase di definizione le linee guida e i criteri per il riconoscimento dei STL } \\
\text { che dovrebbero prevedere forme di aggregazione tra pubblico e privato. }\end{array}$ \\
\hline Campania & - & $\begin{array}{l}\text { In fase di definizione la nuova legge regionale sul turismo, che dovrebbe prevedere } \\
\text { anche i STL. }\end{array}$ \\
\hline Puglia & - & - \\
\hline Sardegna & $\begin{array}{l}\text { LR. 63/1950, DPR } \\
1042 / 1960\end{array}$ & $\begin{array}{l}\text { In fase di definizione il progetto di organizzazione turistica regionale che ad oggi } \\
\text { non prevede un esplicito riferimento ai STL. }\end{array}$ \\
\hline Sicilia & L.R. 9/1986 & $\begin{array}{l}\text { La Regione procede sui due binari: non è stata recepita a135 ma sulla spinta degli } \\
\text { Enti Locali sono stati avviati primi esperimenti di creazioni di STL "spontanei". }\end{array}$ \\
\hline
\end{tabular}

Fonte: Rapporto Turismo, Il Sole 24 ore, 3 giugno 2003, p.2.

È quindi riconosciuto, a livello nazionale, un ruolo rilevante agli approcci sistemici ed al networking nelle destinazioni turistiche. A nostro parere, grazie alle reazioni che ha suscitato questo tipo di approccio negli ultimi anni, anche il legislatore italiano, pur se con strumenti non sempre idonei, ha cercato di sviluppare delle politiche ed iniziative che focalizzano l'attenzione su questo approccio, sia in ambito di progetto che di promozione.

Rispetto al modello proposto del SLT, nonostante l'approccio della legge 135/2001 dedichi una crescente attenzione al territorio e alla effettiva area di riferimento delle destinazioni turistiche, a nostro parere ancora non si considerano sufficientemente gli aspetti sociali dei luoghi in cui esiste questo ispessimento di rapporti fra comunità produttiva e sociale. Questi sono gli elementi principali che rendono il SLT un modello auto-riproduttivo nel tempo. In questo contesto, la 135/2001 rischia di sviluppare strategie e politiche per destinazioni turistiche "deboli" non prendendo in considerazione il grado di presenza della filiera turistica e dell'intensità del processo di valorizzazione delle risorse. D'altro canto l'enfasi posta sui progetti di finanziamento e partnership aiuta a generare 
cooperazione fra gli attori locali e condividere una missione comune, ma un approccio topdown può escludere importanti opinion leaders ed attori economici come già riconosciuto in letteratura (Pechlaner, Weiermair 2000).

\subsection{CONCLUSIONI}

In questo primo capitolo si è presentata una rassegna degli approcci sistemici alle destinazioni turistiche. Dopo un primo panorama dei modelli aziendali si è proposto uno stato dell'arte di quelli territoriali industriali estesi al turismo. Si è cercato di cogliere per ognuno le caratteristiche specifiche ed i vantaggi di analisi. Si è poi proposto un modello il sistema locale turistico (SLT) definito a partire dalla teoria distrettuale. Presentiamo le caratteristiche dei modelli analizzati nella tabella 1.2.

A nostro avviso ognuno dei modelli in tabella ha il merito di focalizzarsi su un aspetto specifico dei sistemi di impresa caratteristico per la fonte di provenienza disciplinare. La totalità degli approcci riesce a rilevare la molteplicità dei vantaggi sistemici di agglomerazione e propone un approccio multidisciplinare allo studio dei sistemi locali.

Il cluster turistico si concentra principalmente sui vantaggi della localizzazione e sulla struttura/rivalità del mercato locale. Inoltre l'unità di analisi è qui il network di imprese e istituzioni associate. Il milieu turistico analizza principalmente l'ambiente socioculturale di un luogo e i networks personali di attori che sviluppano innovazioni e nuove conoscenze.

Il distretto turistico di origine marshalliana-becattiniana è innanzitutto un luogo ed un "ispessimento di interrelazioni" dove il ruolo della comunità sociale è strettamente relazionato con quella industriale. Inoltre, i modelli presentati hanno unità territoriali di riferimento diverse. Il cluster non ha limiti geografici e si può estendere a una città o a una regione. Il distretto è invece, prima di tutto, un luogo circoscritto.

Il sistema locale turistico (STL) da noi presentato si configura come una entità territoriale caratterizzata da due elementi: il cluster di attori economici, non economici ed istituzionali e la comunità sociale ed è prima di tutto un'entità socio-economica dove la comunità di persone coincide con quella lavorativa.

Nel prossimo capitolo individueremo i sistemi locali turistici così definiti utilizzando la metodologia Istat-Sforzi e i sistemi locali del lavoro. Nella seconda parte di questa tesi analizzeremo come competono questi sistemi locali turistici e cercheremo di misurarne le fonti di competitività (economie esterne, spillovers e economie di rete). 
Tabella 1.2: I principali modelli sistemici presentati: caratteristiche principali.

\begin{tabular}{|c|c|c|c|c|}
\hline Modello & Definizione & $\begin{array}{c}\text { Vantaggi/Econo } \\
\text { mie }\end{array}$ & $\begin{array}{c}\text { Origine } \\
\text { principale }\end{array}$ & $\begin{array}{l}\text { Unità di } \\
\text { analisi }\end{array}$ \\
\hline $\begin{array}{l}\text { Destination } \\
\text { Management }\end{array}$ & $\begin{array}{c}\text { Un'area identificata e } \\
\text { promossa ai turisti come un } \\
\text { luogo da visitare e nel quale il } \\
\text { prodotto è coordinato da una } \\
\text { o più autorità o } \\
\text { organizzazione }\end{array}$ & $\begin{array}{l}\text { Gestione e } \\
\text { marketing strategie } \\
\text { di un luogo } \\
\text { immagine, } \\
\text { percezione, visione } \\
\text { sistemica. } \\
\text { (Demand-side) }\end{array}$ & $\begin{array}{c}\text { Pechlaner, } \\
\text { Weissimaier, } \\
\text { Buhalis, Crouch, } \\
\text { Ritchie }\end{array}$ & Destinazione \\
\hline SLOT & $\begin{array}{l}\text { Sistema di ospitalità turistica } \\
\text { specifica e distintiva (attività e } \\
\text { fattori di attrattività)che } \\
\text { valorizza le risorse e la cultura } \\
\text { locali }\end{array}$ & $\begin{array}{l}\text { Visione sistemica, } \\
\text { lungo periodo } \\
\text { coordinato tra } \\
\text { attori. (Supply-side) }\end{array}$ & Rispoli, Tamma & $\begin{array}{l}\text { Località e } \\
\text { attori }\end{array}$ \\
\hline $\begin{array}{l}\text { Sistema } \\
\text { territoriali } \\
\text { Locale } \\
\text { turistico }\end{array}$ & $\begin{array}{l}\text { Sistema territoriale costituito } \\
\text { da una reti locale di soggetti, } \\
\text { un miilieu territoriale, e } \\
\text { l'interazione tra le reti locali e } \\
\text { sovralocali }\end{array}$ & $\begin{array}{l}\text { Ambiente sociale, } \\
\text { Milieu }\end{array}$ & Dematteis & $\begin{array}{l}\text { Luogo e la } \\
\text { rispettiva rete } \\
\text { di attori }\end{array}$ \\
\hline $\begin{array}{l}\text { Cluster } \\
\text { turistico }\end{array}$ & $\begin{array}{c}\text { Gruppo di imprese } \\
\text { interconnesse ed istituzioni } \\
\text { associate }\end{array}$ & $\begin{array}{l}\text { Economie di } \\
\text { localizzazione: } \\
\text { migliore accesso } \\
\text { agli input, rivalità } \\
\text { tra le imprese, } \\
\text { competizione e } \\
\text { cooperazione locale }\end{array}$ & Nordin & Reti di attori \\
\hline Milieu turistico & $\begin{array}{l}\text { Ambiente innovativo, socio- } \\
\text { culturale, network di attori }\end{array}$ & $\begin{array}{l}\text { Innovazioni e } \\
\text { spillovers di } \\
\text { conoscenza tra } \\
\text { attori }\end{array}$ & Peyrache-Gadeaux & $\begin{array}{l}\text { Luogo e la } \\
\text { rispettiva rete } \\
\text { di attori }\end{array}$ \\
\hline $\begin{array}{l}\text { Distretto } \\
\text { turistico }\end{array}$ & $\begin{array}{l}\text { uno stabile e ragionevole } \\
\text { ispessimento di relazioni tra } \\
\text { un sistema di imprese } \\
\text { turistiche nel tempo }\end{array}$ & $\begin{array}{c}\text { Economie esterne } \\
\text { mashalliane }\end{array}$ & Corigliano & $\begin{array}{l}\text { Luogo } \\
\text { definito }\end{array}$ \\
\hline $\begin{array}{l}\text { Sistema locale } \\
\text { Turistico } \\
\text { (SLT) }\end{array}$ & $\begin{array}{l}\text { un'entità socio-economica } \\
\text { coincidente con una comunità } \\
\text { sociale ed una popolazione di } \\
\text { imprese } \\
\text { (Cluster di attori economici, } \\
\quad+\text { comunità sociale) }\end{array}$ & $\begin{array}{l}\text { Economie esterne, } \\
\text { rivalità, ambiente } \\
\text { socio-economico, } \\
\text { economie di rete, e } \\
\text { spillovers }\end{array}$ & - & $\begin{array}{l}\text { Entità socio } \\
\text { economica } \\
\text { (SLL) }\end{array}$ \\
\hline
\end{tabular}

Fonte: nostra elaborazione; 


\section{CApitolo II}

\section{IDENTIFICAZIONE ED ANALISI DEI SISTEMI LOCALI TURISTICI IN ITALIA}

\subsection{PREMESSA}

Lo scopo di questo capitolo è di proporre una metodologia per l'identificazione territoriale dei sistemi turistici italiani, definita a partire dai sistemi locali del lavoro (ISTAT, 1997). L'identificazione territoriale dei sistemi turistici è stata fino ad ora di tipo indiretto, cioè derivata da altre classificazioni territoriali di carattere economico (sistemi locali manifatturieri, etc.). Si partiva dall'individuazione dei sistemi locali turistici industriali e/o commerciali per poi evidenziare luoghi turistici esclusivamente specializzati in attività turistiche.

In queste pagine cerchiamo invece di interrogarci su quali siano i sistemi locali turistici in Italia e come individuarli. In questo processo focalizziamo la nostra attenzione sugli attori di un sistema locale turistico e sulla conseguente struttura dell'offerta; verifichiamo poi se i SLT individuati coincidono con l'ampio panorama delle destinazioni turistiche italiane. Si propone delle soluzioni a questi problemi riflettendo anche sulla metodologia utilizzata e alla sua applicabilità all'industria turistica e ponendo attenzione alla variabilità dei risultati in base alle differenti definizioni di turismo che si adottano.

Nel prossimo paragrafo presenteremo un breve panorama dell'identificazione dei sistemi turistici italiani attraverso i principali studi effettuati. Nel terzo paragrafo descriveremo invece la fonte utilizzata per la nostra metodologia di analisi. Nel quarto e quinto esporremo i risultati di due studi basati sul censimento intermedio del 1996 e su quello del 2001.

Analizziamo il comparto turistico e cerchiamo prima di tutto di proporre un panorama dell'evoluzione di tale industria durante gli ultimi dieci anni. Nel grafico sottostante è evidenziata la crescita dell'occupazione dal 1991 al 2001 a livello complessivo italiano, per il settore HoReCa (Hotel, Ristoranti e Caffè), per una filiera turistica di tipo allargato (trasporti, alloggiamento, attività ricreative, servizi ai turisti, etc. ${ }^{45}$ ) e dei servizi in generale.

La figura 2.1 sottolinea come a differenza di una crescita di circa il $7 \%$ dell'occupazione totale, il settore $\mathrm{HoReCa}^{46}$ (hotel, ristoranti e caffè) cresce di quasi il doppio, circa il 15\%, mentre le attività all'interno della filiera aumentano del 23,5\% nel periodo considerato. Questa crescita coincide con quella dei servizi e con il fenomeno più generale della terziarizzazione dell'economia, evidenziato dall'incremento del 23,3\% del settore terziario in generale. Questo panorama sottolinea la rilevanza delle attività turistiche e l'importanza di questa industria all'interno dell'economia italiana.

\footnotetext{
45 Presenteremo una descrizione approfondita di questa filiera nel quinto paragrafo nella tabella 4.4.

${ }^{46} \mathrm{La}$ voce è presentata dettagliatamente in appendice e in maniera più approfonditamente più avanti in questo capitolo.
} 
Figura 2.1: La crescita dell'occupazione in Italia, nel settore $\mathrm{HoReC}_{0}$, nella Filiera ${ }^{47}$ e nei Servizi;

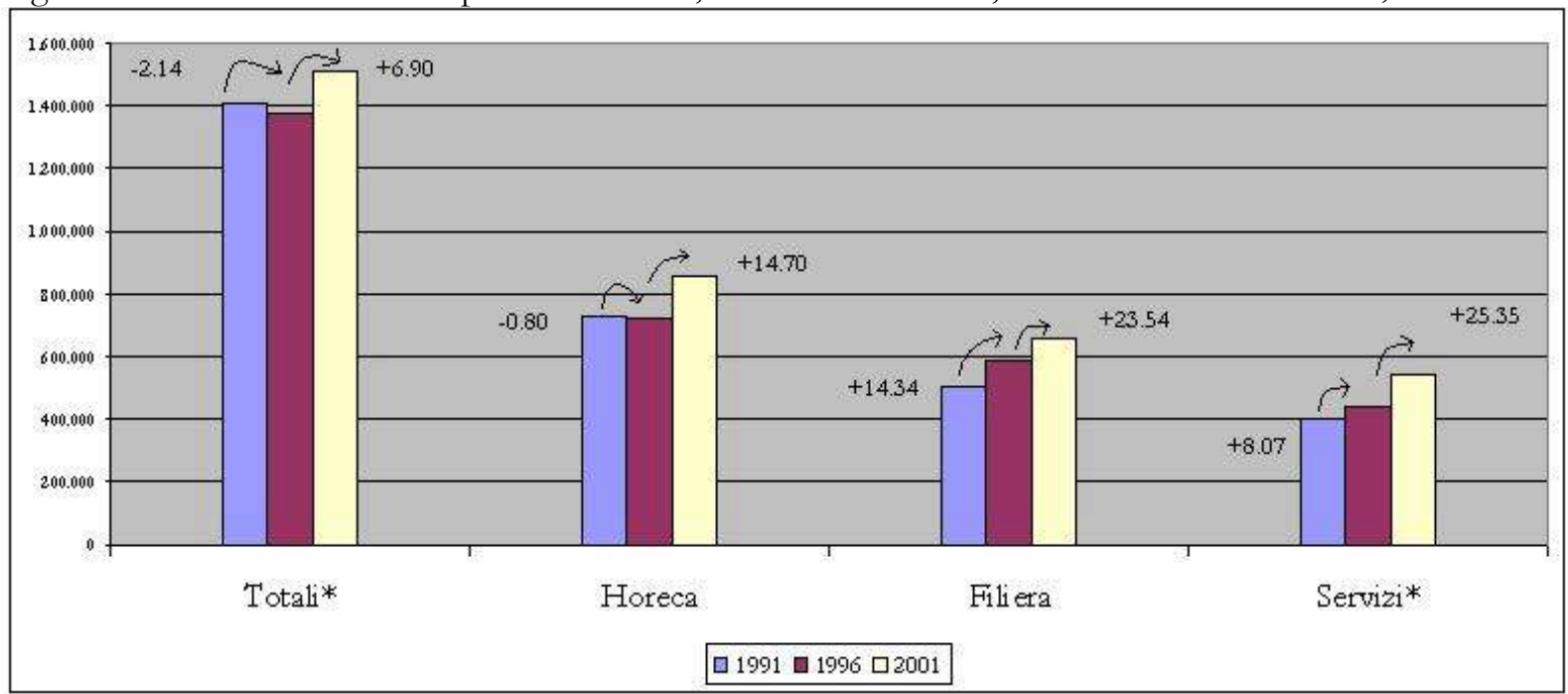

Fonte: ns. elaborazione su dati ISTAT 1991, 1996, 2001.

$*$ in decine

\subsection{LO STATO DELL'ARTE: L'INDIVIDUAZIONE DEI SLT IN ITALIA}

In queste pagine proponiamo una rassegna sull'individuazione territoriale dei sistemi locali turistici in Italia. In particolare cerchiamo di richiamare quei contributi che hanno un approccio quantitativo e/o geografico e che hanno proposto analisi allargate al territorio italiano o regionale.

Un'analisi essenziale alla studio applicato sui distretti (industriali) è quella relativa all'individuazione dei sistemi locali del lavoro. Questo studio, proposto da Sforzi (1987, 1997a) e curato nel noto lavoro "I sistemi locali del lavoro 1991", riporta una classificazione territoriale di tipo non amministrativo, relativa ad aggregati di comuni individuati da un apposito algoritmo di regionalizzazione basato sui lavoratoti residenti nell'area. Questi raggruppamenti di comuni sono denominati sistemi locali del lavoro (SLL). ${ }^{48}$.

Analisi successive hanno reso possibile costruire una mappa dell'Italia in base ai sistemi locali di servizio turistico in senso stretto (ristoranti, agenzie di viaggio, ecc.) (Sforzi, 1997b); in questo contributo si analizza in particolare lo sviluppo dei sistemi urbani italiani in ottica settoriale. L'autore analizza infatti l'evoluzione dal 1981 al 1991, in base ai censimenti ISTAT, dei sistemi locali del lavoro suddivisi in base alle seguenti categorie: industriali, commerciali, servizi alle imprese e persone, servizi esclusivi alle persone (alberghi, ristoranti, bar) $^{49}$ (figura 2.2).

\footnotetext{
${ }^{47}$ Filiera costruita senza le attività artigianali $(1.13,2.16)$ e i trasporti ferroviari $(60.1)$ che includono i trasporti su merci.

${ }^{48}$ Nei sistemi locali del lavoro la popolazione condivide esperienze di lavoro e di vita che sono alla base del concetto del distretto industriale e della sua identificazione; tramite i noti coefficienti di concentrazione territoriale.

${ }^{49} \mathrm{Ci}$ si riferisce alla voce $\mathrm{H}$ del codice Ateco 91 con la quale si è replicata l'elaborazione in base al censimento intermedio del 1996. Per qualsiasi tipo di approfondimento sulla definizione delle classificazioni analizzate rimandiamo agli approfondimenti in appendice.
} 
Figura 2.2: Mappa dei sistemi locali dei servizi al consumatore, 1991;

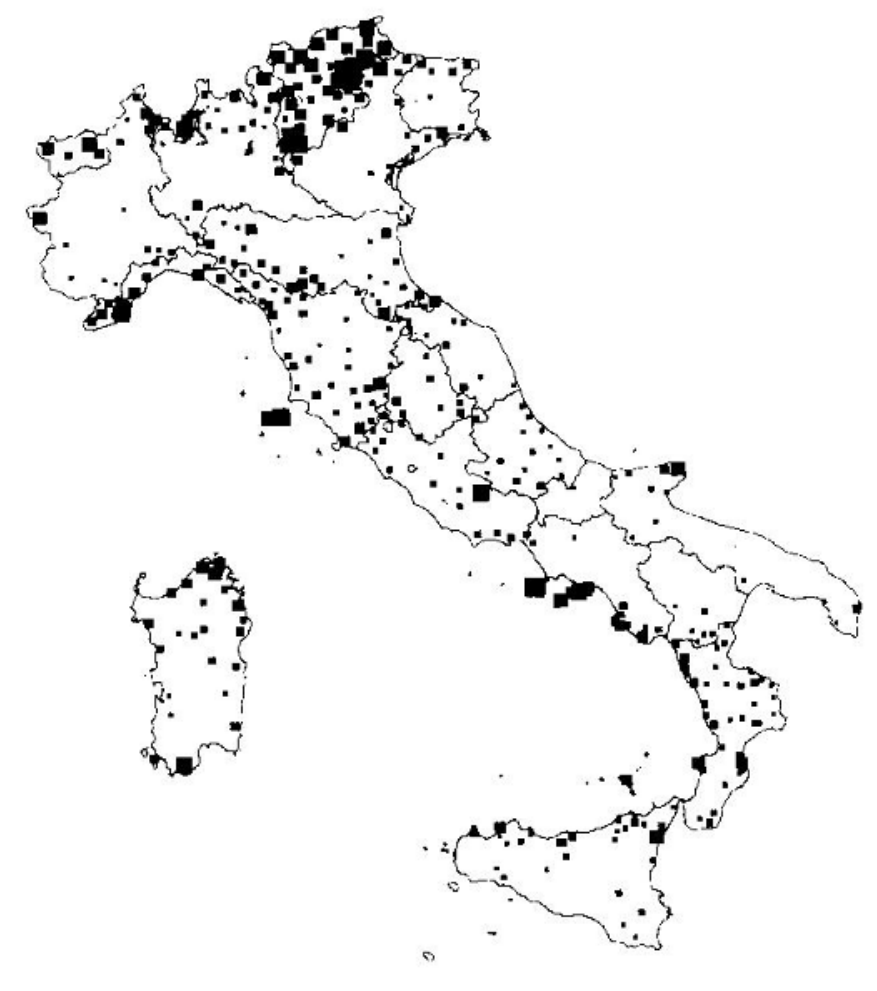

Valori del coefficiente di concentrazione territoriale

Minimo • 1,01

Massino 8.22

Fonte: Sforzi, 1997b;

Questo studio risulta di indubbio interesse al fine del raggiungimento del nostro scopo, tuttavia ancora non si misurano sistemi turistici, ma sistemi specializzati nei servizi alle persone. Da questo punto di vista, riteniamo sia necessario ampliare la definizione di servizi alle persone in senso "turistico".

L'ISTAT solo recentemente propone alcune analisi dei SLL, ma con poche variabili molto aggregate e prodotte come stime della Contabilità Nazionale (PIL, occupazione, popolazione). Inoltre tale classificazione è spesso suddivisa in base al comparto caratterizzante del sistema: industriale, di servizi e misto. L'analisi ISTAT è sviluppata tramite una cluster analysis per le attività prevalenti dell'area locale ad un livello di tre digits.

Tale analisi, mentre risulta di una certa importanza per i sistemi industriali e per la loro evoluzione temporale, non lo è, a nostro parere, riguardo ai sistemi locali turistici in quanto registra solo i sistemi puri escludendo $\mathrm{i}$ sistemi che contribuiscono ad altre industrie. Presentiamo la mappa dei sistemi locali turistici elaborata dall'ISTAT nel 1996 in base al censimento dello stesso anno.

La mappa registra solo quei sistemi locali turistici che hanno una specializzazione prevalente ed esclusiva nel comparto turistico, escludendo dall'analisi le varie relazioni con gli altri settori. 
Figura 2.3: Distretti turistici secondo l'ISTAT;

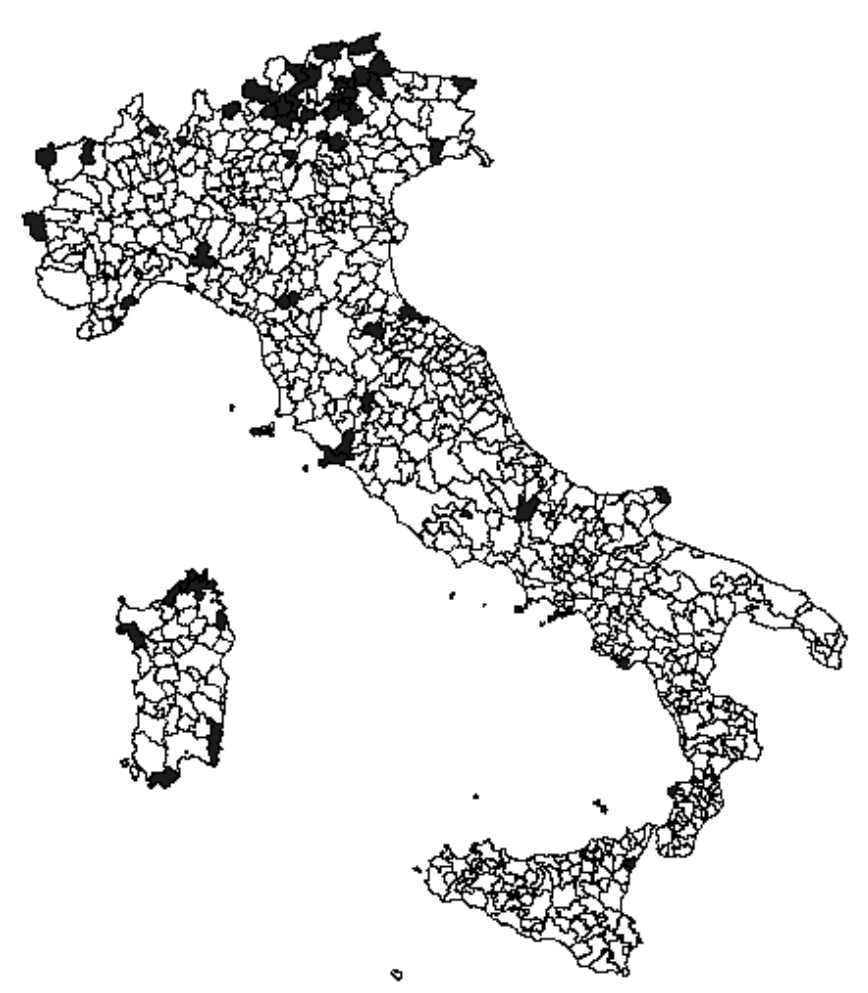

Fonte: nostra elaborazione su dati Istat, 1996;

Un altro importante contributo al concetto dei sistemi economici turistici è fornito dall'Istituto Regionale per la Programmazione Economica della Toscana (IRPET), prima con il libro di Cavalieri (1999) "Toscana e Toscane" e successivamente con "Sistemi Locali in Toscana" di Bacci (2002). L'analisi si basa sui Sistemi Economici Locali (SEL) costituiti con la delibera regionale nr. 219 del 1999. Lo studio approfondisce il legame tra territorio e sistemi produttivi locali e perviene alla costruzione di una tassonomia di sistemi locali, tramite l'utilizzo delle tavole input-output per SEL ${ }^{50}$.

Tre sono i sistemi locali turistici individuati da Bacci (2002), quelli turisticoindustrali, turistico-aperti e turistico-rurali. I primi sono caratterizzati dalla compresenza di attività turistiche ed industriali. In queste aree, l'equilibrio tra turismo ed industria non è sempre stabile e lo sviluppo nel tempo può portare ad una caratterizzazione di un sistema produttivo a scapito dell'altro (Val di Cornia, val di Cecina, Massa Carrara, casentino). Il secondo è quello dei sistemi turistici aperti caratterizzato, oltre dalla presenza di attività industriali, da un saldo positivo nei consumi turistici (sistemi locali della costa, Chianciano, ecc.). L'ultimo gruppo è quello denominato dei sistemi turistici rurali, che individua quelle aree che hanno avuto solo una leggera industrializzazione negli anni 60-70 e presentano dei medi livelli di consumo turistico. (aree attorno a Siena, Amiata, ecc.). Nella figura 2.4 ne evidenziamo i risultati.

\footnotetext{
${ }^{50}$ L'analisi viene effettuata calcolando la prevalenza del saldo import-export per attività. Rimandiamo al testo di Bacci (2002) per ulteriori approfondimenti.
} 
Figura 2.4: I sistemi economici locali turistici nella regione Toscana;

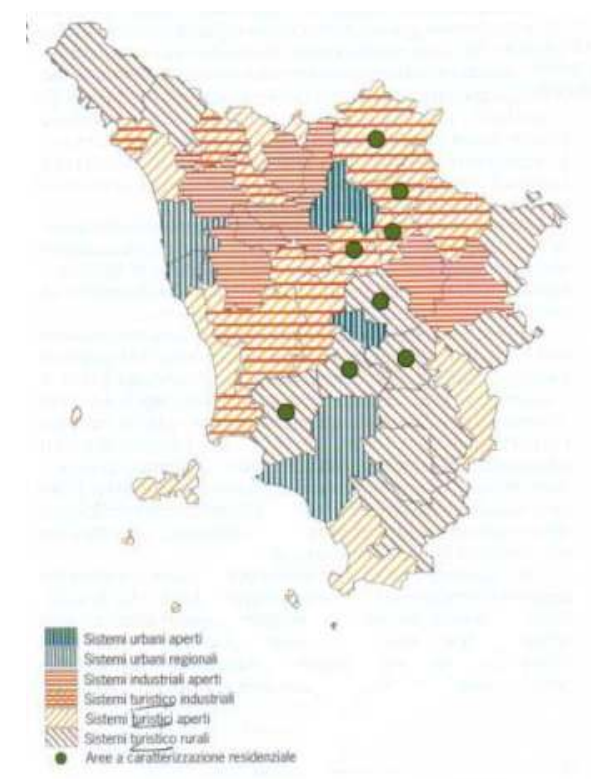

Fonte: Sistemi Locali della Toscana (Bacci, 2002:264)

Il contributo di Bacci risulta interessante ai fini della nostra analisi, tuttavia lo scopo dell'autore è la classificazione dei SEL in Toscana e non l'individuazione dei SLT. Da questo punto di vista non è misurato il peso del turismo nei sistemi o l'eventuale concentrazione di imprese, ma solo una sua presenza sostanziale.

Lo studio già ricordato dell'ACI-CENSIS individua i distretti turistici nel territorio italiano attraverso sette indicatori. Questi gli indici utilizzati: grado di terziarizzazione (indicatore sul quale è stato costruito il rating); quadro di evoluzione dell'offerta di servizi (in relazione alle vocazioni primarie ed alle opportunità di divertimento, shopping, sport); qualità della ristorazione (concentrazione punti di ristorazione di alta qualità); qualità dell'ospitalità (concentrazione di alberghi segnalati dalle guide più importanti); segmentazione ristorazione (grado di diversificazione dell'offerta gastronomica; segmentazione ospitalità (grado di diversificazione dell'offerta alberghiera: numero di stelle); antinomia omogeneità su integrazione (intreccio delle vocazioni dei diversi comuni che costituiscono il singolo distretto), "plus enogastronomico".

Il rapporto registra 299 distretti turistici che risultano individuati in base alle caratteristiche della offerta e della domanda, tuttavia il concetto di distretto utilizzato non coincide con quello tradizionale economico-industriale e si basa maggiormente sull'individuazione di determinate tipologie di agglomerazioni di imprese (alberghi e ristoranti di alta qualità, etc.). Nella figura numero 2.5 presentiamo i risultati dello studio dell'ACI-CENSIS. 
Capitolo secondo

Tabella 2.1: I distretti del rapporto ACI-CENSIS.

\begin{tabular}{|l|c|c|c|c|c|c|}
\hline Distretti Turistici & Nr. distretti & Comuni & Abitanti* & Ristoranti & Alberghi & Presenze* \\
\hline Marini & 96 & 777 & 10,3 & 7.000 & 9.200 & 96 \\
\hline Artistico-culturali & 37 & 273 & 5,3 & 3.500 & 3.700 & 39 \\
\hline Montani & 137 & 1.504 & 4,6 & 4.500 & 9.700 & 54 \\
\hline Intergrati & 29 & 287 & 2,2 & 1.600 & 1.700 & 15 \\
\hline Totale & 299 & 2841 & 22,4 & 16.000 & 24.300 & 204 \\
\hline
\end{tabular}

Fonte: Rapporto Aci-Censin.

$*$ in milioni

Figura 2.5: Mappa dei distretti turistici;

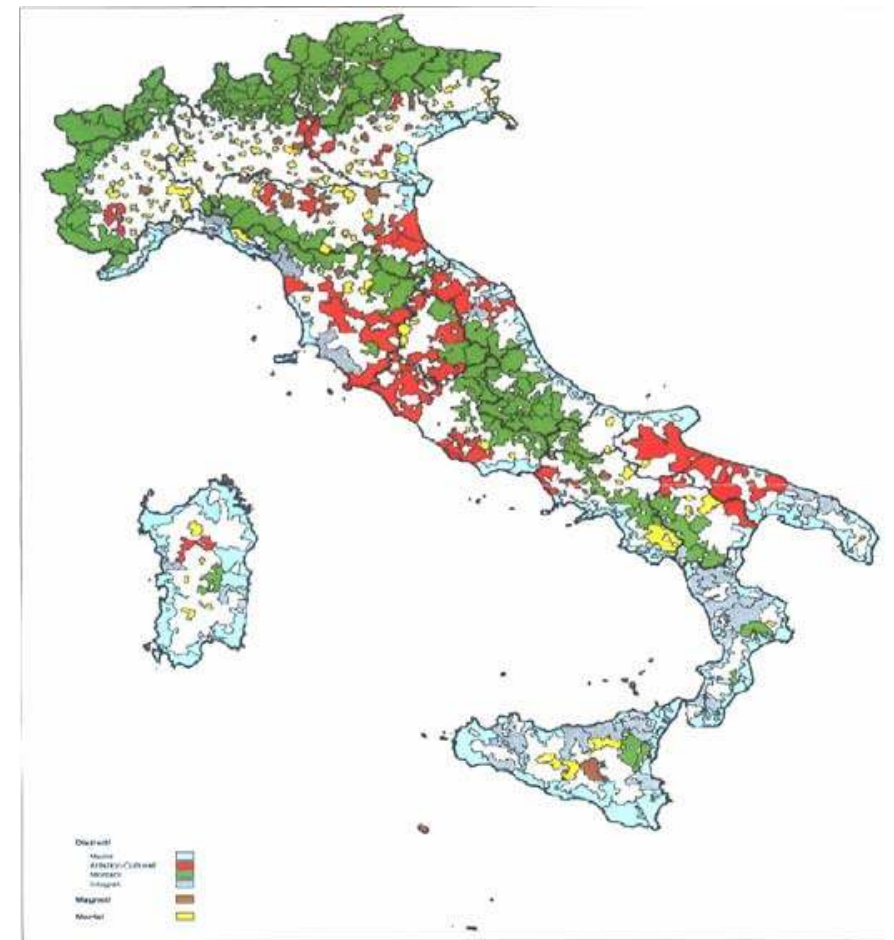

Fonte: ACI-Censis, 2002; 


\subsection{LA FONTE DEI DATI}

Lo scopo di questo capitolo è quello di presentare una metodologia di identificazione quantitativa dei sistemi turistici italiani basata sui sistemi locali del lavoro. Come già evidenziato nel primo capitolo, l'identificazione dei sistemi turistici o di eventuali distretti turistici è sempre stata sviluppata in maniera derivata, partendo dall'individuazione dei sistemi locali turistici industriali e/o commerciali si evidenziava i soli luoghi turistici. In queste pagine, cercheremo invece di identificare i sistemi locali turistici e di evidenziare quali sono i legami e le interrelazioni di un luogo con una forte industria turistica con gli altri settori e se i SLT individuati raffigurano l'ampio panorama italiano delle destinazioni turistiche. Nel corso del capitolo si cerca di proporre soluzioni a tali problemi, riflettendo anche sulla metodologia utilizzata e alla sua applicabilità all'industria turistica ed, in particolare, alla variabilità dei risultati in base alle diverse definizioni di turismo che si adottano.

Nelle analisi che seguono utilizzeremo come unità di analisi territoriale i Sistemi Locali del Lavoro (SLL) del 1991 per i seguenti ordini di motivi (Menghinello, 2002:12):

a) I SLL permettono di superare la ripartizione di tipo amministrativo e si riferiscono maggiormente all'effettiva organizzazione industriale del territorio;

b) Sono unità territoriali maggiormente idonee ad analisi basate su aspetti economici e sociali essendo riferiti all'intensità tra residenti e lavoratori di un luogo;

c) Rispettano una rigorosa metodologia d'individuazione (per ogni approfondimento si rimanda al contributo sui Sistemi Locali del Lavoro, 1997).

Anche se i SLL sono stati identificati in base agli spostamenti dei lavoratori nelle aggregazioni dei 8100 comuni nel 1991 e quindi identificati circa un decennio fa, pensiamo che siano l'unità di analisi maggiormente idonee per evidenziare una concentrazione di imprese anche ai giorni nostri, sia perché l'evoluzione di un sistema locale è un processo temporalmente ampio, sia perché le relazioni sociali ed economiche di un luogo sono abbastanza stabile nel tempo. Infine, i nuovi SLL costruiti in base al censimento del 2001 saranno resi disponibili dall'ISTAT non prima del primo trimestre 2005.

Le analisi sviluppate nel capitolo si basano su una fonte di dati costruita ad hoc aggregando ed integrando molteplici database resi disponibili dalle principali istituzioni informative italiane. In particolare la raccolta dati principale è stata effettuata nel primo trimestre del 2004 sul database ASIA (Archivio Imprese Attive) in base ad uno stage effettuato all'ISTAT presso il Dipartimento di Statistiche Strutturali per le imprese (DSS) ${ }^{51}$. Questi dati sono stati poi aggregati con le statistiche ufficiali pubblicate dall'ISTAT dei vari dipartimenti (Statistiche sul Turismo, SLL, etc.) e quelle di altre istituzioni tra quali l'Unione Italiana Cambi (UIC), che registra trimestralmente le spese turistiche degli italiani e degli stranieri.

Le principali fonti dei dati utilizzati nell'analisi dei sistemi locali del lavoro sono le seguenti:

\footnotetext{
51 Lo stage che si è svolto da gennaio e marzo 2004 è stato possibile grazie ad una Convenzione ad hoc sviluppata tra l'Università di Firenze, Dipartimento di Scienze Aziendali e l'Istituto di statistica nazionale (ISTAT). Inoltre, sono stati necessari due tutor di riferimento nel periodo, oltre, alla Prof.ssa Lazzeretti, supervisor del Dottorato EGISL, si è affiancato, il dott. Stefano Menghinello del DSS.
} 
1. Censimento ISTAT 1991; 1996; 2001, disponibile a marzo 2004. Censimento delle Unità Locali ripartite per comuni e successivamente riclassificate per sistemi locali del lavoro del 1991 (disponibile on-line);

2. Ricettività alberghiera (letti e camere) per COMUNI, fonte ISTAT 2001, pubblicazione STATISTICHE SUL TURISMO;

3. Arrivi e presenze (italiani e stranieri) suddivise per esercizi e per PROVINCIE (anche per APT (547 in Italia) sub-comunale), fonte ISTAT 2001, pubblicato su STATISTICHE DEL TURISMO;

4. Spesa degli stranieri in Italia per PROVINCIA visitata, fonte: UIC 1999-2002;

5. Spesa degli italiani all'estero per PROVINCIA di residenza, fonte: UIC 1999-2002;

6. Viaggiatori italiani all'estero per PROVINCIA di residenza, fonte: UIC 1999-2002;

7. Indici generali sui Sistemi Locali, (popolazione, kmq, addetti, etc.): ISTAT.

Tabella 2.2: variabili economiche utilizzate e dettaglio territoriale di riferimento;

\begin{tabular}{|l|l|l|}
\hline Variabile economica & $\begin{array}{l}\text { Dettaglio territoriale di } \\
\text { riferimento }\end{array}$ & Fonte dei dati \\
\hline $\begin{array}{l}\text { Addetti e Unità Locali (Censimento } \\
1991,1996,2001)\end{array}$ & Comunale & $\begin{array}{l}\text { ISTAT Censimento, 1991, } \\
\text { 1996, 2001 }\end{array}$ \\
\hline Letti e Camere - Letti e Camere & Comunale & $\begin{array}{l}\text { ISTAT, Statistiche sul } \\
\text { Turismo }\end{array}$ \\
\hline Arrivi e presenze (italiani e stranieri) & Provinciale & UIC \\
\hline Spesa turistica (Italia e estero) & Provinciale & UIC \\
\hline Viaggiatori italiani all'estero & Provinciale & UIC \\
\hline Indici generali SLL & Sistema Locale del Lavoro & ISTAT 2002 \\
\hline
\end{tabular}

Fonte: nostra elaborazione;

Le elaborazioni sono state effettuate a livello di sistema locale ed è stato necessario armonizzare i dati territoriali, poiché i dati relativi ai punti 3, 4, 5 e 6 sono registrati a livello provinciale (spesa turistica provinciale, arrivi e presenze, viaggiatori italiani all'esterno per provincia di residenza). Inoltre gli arrivi e le presenze (nr. 3) sono registrate a livello di circoscrizione turistica, quindi sub-provinciale. Risulta però difficile legare questo ambito territoriale con i SLL, dato che le 547 circoscrizioni turistiche sono aggregazioni di uno o più comuni non coincidenti con i sistemi locali.

Al fine di armonizzare i dati provinciali si è proceduto con il seguente metodo, si è ripartito ogni dato provinciale per comune e successivamente si è aggregato ogni comune per sistema locale. La ripartizione dei dati provinciali in unità territoriali comunali è stata effettuata tramite un indice costruito in base ai lavoratori nell'industria turistica ${ }^{52}$ di ogni comune, come in Deidda et al. (2000) e in Paci ed Usai (1999):

$$
I_{i}=\frac{E_{j}}{E_{i}}
$$

\footnotetext{
52 Per la definizione di "turismo" rimandiamo più avanti in questo stesso capitolo.
} 
dove l'indice di distribuzione del comune $\mathrm{I} i$ è costruito in base al rapporto tra gli addetti della provincia $j$ e gli addetti del comune $i$. Si è quindi ipotizzato che all'interno della provincia vi sia un certo grado di omogeneità nelle preferenze dei visitatori e che queste si distribuiscono in base all'offerta (gli addetti). In altre parole la spesa, le presenze e gli arrivi nel comune $i$ sono presunti in modo direttamente proporzionale al numero di addetti nel turismo in quel comune in confronto alla media provinciale.

\subsubsection{L'unità di analisi territoriale: i sistemi locali del lavoro}

Il primo problema che s'incontra nell'identificazione concreta di un concetto teorico e di riuscire a definirne le misure e i pesi. Il distretto industriale, nella sua storia, si è scontrato con la difficoltà d'analisi quantitativa. Inoltre una concettualizzazione di questo tipo ha sempre avuto difficoltà a trovare un riconoscimento tra i confini amministrativo geografici. Il distretto industriale, per definizione, sovrasta questi confini e nella realtà spesso è un insieme di più comuni limitrofi ${ }^{53}$.

In questo panorama lo sforzo dei primi studiosi dei distretti industriali è stato di dare un riconoscimento territoriale ad un concetto che con il territorio era legato strettamente e non ne poteva prescindere. La metodologia utilizzata per identificare nel territorio il distretto industriale è stata la seguente. Il territorio nazionale, esaminato in prima approssimazione solo in Toscana (IRPET, 1987) fu ripartito in unità territoriali definite dagli spostamenti giornalieri, per motivo di lavoro, effettuati dalla popolazione residente in una tipica giornata lavorativa. Queste unità sono note da allora come sistemi locali del lavoro (Istat, 1997a). Si fu in grado di generare così un "tessuto localizzato di relazioni socio-economiche, fra i diversi membri della società locale, tale da favorire la formaz̨ione, la diffusione e il mantenimento di un sistema di valori, di conoscenze produttive, di comportamenti tipici e d'istituzioni attraverso $i$ quali la società locale interagisce con l'organizzazione produttiva" (Becattini e Sforzi, 2002:21) che è tipica di un sistema di produzione locale. Il sistema locale del lavoro si configurò così in una grande città, in un sistema rurale o in un sistema turistico in relazione dalla specializzazione del suo tessuto produttivo.

Il quesito sullo spostamento giornaliero per motivi di lavoro o di studio fu introdotto dall'ISTAT per la prima volta nel 1971, ma raggiunse un consistente valore analitico solo nel 1991, con l'utilizzo di un vero sistema informativo geografico e grazie alla possibilità di utilizzare un maggior numero di dati con variabili georeferenziate (Istat-Sforzi, 1997a). Con tale metodologia, in prima istanza, si riuscì a ripartire il territorio nazionale, secondo criteri maggiormente socio-economici, in sistemi locali del lavoro. In seguito, attraverso l'applicazione di quelli che furono chiamati $i$ coefficienti di concentrazione territoriale, si riuscì a costruire una mappa dell'Italia dei distretti industriali (ISTAT, 1997a).

I censimenti hanno sempre privilegiato scale nazionali o regionali per la raccolta dei dati, mentre gli scienziati sociali hanno comunque sempre cercato di avere un approccio invece continuamente bottom-up e quindi di partire dalla realtà locale per poi arrivare alla scala nazionale. Il concetto guida utilizzato è stato quello di concentrare attività produttive e servizi in quantità tali da offrire opportunità di lavoro e residenziali alla maggior parte della popolazione che vi abita, concetto denominato poi come autocontenimento. Questo indica per un territorio "la sua capacità di comprendere al suo interno la maggiore quantità di relazioni umane che intervengono fra le sedi dove si svolgono le attività di produrione (località di lavoro) e quelle dove

53 Si veda per esempio Dei Ottati 1995, che identifica il distretto Pratese come l'unione di 9 comuni della zona: quali Agliana, Montale, Cantagallo, Carmignano, Montemurlo, Poggio a Caiano, Prato, Vaiano e Vernio. 
si svolgono le attività legate alla riprodurione sociali" (Istat-Sforzi, 1997:19 e ss.). Un territorio di questo tipo venne appunto denominato sistema locale del lavoro: "un'entità socio-economica che comprende occupazione, acquisti, ricreazione, e opportunità sociale.. ... data una base residenżiale individuale e la necessità di farvi ritorno alla fine della giornate" ${ }^{34}$.

La metodologia dell'individuazione dei sistemi locali portò alla configurazione geografica dei 784 SLL costituiti dall'ISTAT nel 1991. Essi furono il risultato dall'aggregazione tramite autocontenimento a livello del 75\% degli spostamenti giornalieri di lavoro degli 8100 comuni italiani designati con il censimento del 1991.

Dopo questa breve disamina, emerge come questa metodologia sia idonea nella configurazione geografica anche di un sistema locale turistico. Infatti l'oggetto finale della nostra analisi, il SLT, è prima di tutto un luogo dove persone condividono esperienze di vita e di lavoro con una certa stabilità nel tempo, quindi prima di tutto un sistema locale in senso proprio. Nei prossimi paragrafi cercheremo di applicare dei coefficienti (turistici) di concentrazione territoriale ai sistemi locali del lavoro del 1991.

Nonostante il lasso di tempo trascorso e l'innegabile evoluzione alla quale un'area locale è soggetta, riteniamo che l'utilizzo dei sistemi locali del lavoro 1991 possa configurarsi come una prima approssimazione di analisi empirica di sistemi locali caratterizzati dall'industria turistica. Inoltre pensiamo che la vita sociale e le relazioni socioeconomiche di un sistema locale siano delle caratteristiche che registrano una certa continuità nel tempo. Inoltre, come già detto, la possibilità di utilizzare dati georeferenziati da parte dell'ISTAT è valida, al momento, solo per i sistemi locali costruiti con il censimento del 1991.

In sintesi, indichiamo i coefficienti di specializzazione territoriale utilizzati nel volume dell'ISTAT (1997a). La metodologia di individuazione dei Distretti Industriali adottata nel "Rapporto annuale. La situazione del Paese nel 1995" (Istat, 1996:261-268) assume come unità territoriale d'analisi il sistema locale del lavoro (Istat, 1997a) e prevede i seguenti stadi:

1) individuazione dei sistemi locali che sono manifatturieri;

2) individuazione dei sistemi locali manifatturieri che sono di piccola e media impresa (PMI);

3) individuazione dell'industria principale di ciascun sistema locale manifatturieri di PMI;

4) individuazione (come Distretti Industriali) dei sistemi locali manifatturieri che sono di PMI la cui industria principale è costituita da PMI.

Gli indici territoriali di specializzazione hanno fornito una mappa dell'Italia corrispondente approssimativamente alla Terza Italia di Bagnasco (Sforzi, 1987). Infine, la mappa costruita dall'ISTAT (Istat-Sforzi, 1997a) è nota a chi si occupa di distretti industriali e ha permesso che il concetto di distretto industriale avesse un maggior riconoscimento anche a livello teorico (Becattini, 2000).

\footnotetext{
${ }^{54}$ Per approfondire l'approccio metodologico alla costruzione dei sistemi locali del lavoro e il concetto dell'autocontenimento si veda ISTAT 1997.
} 


\subsection{Primo Studio: Il Censimento inTermedio DeL 1996 ED IL} SETTORE HORECA

Seguendo l'approccio descritto e partendo dai sistemi locali del lavoro del 1997, si può verificare la specializzazione sociale e produttiva di tipo turistico dei diversi luoghi. L'obiettivo è di individuare quei sistemi locali classificandoli secondo le caratteristiche delle unità produttive, prevalentemente di piccola e media dimensione ${ }^{55}$, con una maggiore specializzazione nel comparto turistico in confronto alla media nazionale. Il primo problema è quindi quello di definire cosa è turistico e cosa non lo è. Si possono valutare solo le imprese direttamente coinvolte con il turismo (alberghi, campeggi, agenzie di viaggio) oppure anche quelle indirettamente collegate (agenzie di trasporto, affitto automobili) oppure quelle che sono anche collegate alla normale vita di tutti i luoghi (bar, ristoranti, caffè). Per semplificazione metodologica, adotteremo in questo primo studio la definizione dell'ISTAT della voce $\mathrm{H}$ - Hotel e Ristoranti. La voce, $\mathrm{H}^{56}$ include tutte le voci descritte nella tabella 2.3 .

Tabella 2.3: Voce H. Hotel e Ristoranti - Codice Ateco;

55.11.0 Alberghi e motel, con ristorante
55.12.0 Alberghi e motel, senza ristorante
55.21.1 Ostelli della gioventù
55.21.2 Rifugi di montagna
55.22.0 Campeggi ed aree attrezzate per roulette
55.23.1 Villaggi turistici
55.23.2 Colonie, case per ferie e case di riposo (senza cure mediche)
55.23.3 Gestione di vagoni letto
55.23.4 Affittacamere per brevi soggiorni, case per vacanze
55.23.5 Agriturismo
55.23.6 Altri esercizi alberghieri complementari (compresi i residence)
55.30.1 Ristoranti, trattorie, pizzerie, osterie e birrerie con cucina
55.30.2 Rosticcerie, friggitorie, pizzerie a taglio con somministrazione
55.30.3 Gestione di vagoni ristorante
55.30.4 Servizi di ristorazione in self-service
55.30.5 Ristoranti con annesso intrattenimento e spettacolo
55.40.1 Bar e caffè
55.40.2 Gelaterie
55.40.3 Bottiglierie ed enoteche con somministrazione
55.40.4 Bar, caffè con intrattenimento e spettacolo
55.51.0 Mense
55.52.0 Fornitura di pasti preparati

Fonte: Ateco91;

Anche la OECD (1990) riconosce la forza lavoro nel settore turistico come appartenente principalmente a tre settori principali e rifacendosi alla classificazione internazionale $I S I C$ a due digits classifica le attività connesse al turismo in:

- 55 hotel, ristoranti e caffè;

- 60-63 trasporti e agenzie di viaggi;

- $92 \quad$ Attività ricreative culturali e sportive.

55 Minore di 250 addetti.

56 Vedi in appendice il contenuto specifico di queste voci. 
Naturalmente non tutti i posti di lavoro creati in queste industrie possono essere attribuiti al turismo. Abbiamo tuttavia deciso di riferirci prevalentemente al settore HORECA (hotel, ristoranti, caffè) che è il gruppo più strettamente collegato al turismo e maggiormente omogeneo a livello europeo e che registra una certa rilevanza nella ricerca (Commissione Europea, 2003). Pensiamo che tale semplificazione, quindi, possa essere abbastanza verosimile alle principali attività turistiche di una zona. In altre parole ci aspettiamo una maggiore specializzazione in queste attività in una zona turistica.

L'ISTAT raccoglie nel Censimento intermedio dell'industria e dei servizi del 1996 questi dati per ogni comune, l'unità d'analisi più piccola disponibile. Si possono quindi aggregare i dati comunali della voce $\mathrm{H}$, Alberghi e Ristoranti, per i sistemi locali del lavoro, costruiti in base al censimento del 1991. Queste unità territoriali superano i meri confini amministrativi e integrano comuni che condividono lo stesso tessuto di relazioni socio-economiche, come già visto.

Il passo successivo è quello di applicare gli indici di concentrazione territoriale ai 784 sistemi locali del lavoro esistenti. Questi gli indici di concentrazione territoriale turistica che utilizziamo:

1. Sistemi locali turistici. Il primo indice evidenzia quei sistemi locali che hanno una maggiore specializzazione nel comparto turistico, così calcolato:

Indice specializzazione turistica $=L Q_{i s}=\frac{E_{i s}}{E_{s}} / \frac{E_{i}}{E}$

dove $E_{i s}$ è il numero di addetti nelle unità locali nel sistema locale $s$ specializzato nel settore $i$ (HoReCa sector); $E_{s}$ è il numero di addetti nelle unità locali nel sistema locale $s$; $E_{i}$ è il numero di addetti in Italia specializzati nel settore $i$ (HoReCa sector); e E è l'occupazione totale in Italia.

Questo tasso indica la maggiore specializzazione sulla media nazionale del sistema locale per quanto riguarda il settore $\mathrm{HoRe}_{\mathrm{C} a}$ dell'ISTAT.

2. Sistemi locali che sono di piccola impresa: Il secondo indice individua quei sistemi locali che sono caratterizzati da piccole e medie imprese con un numero di addetti inferiori a 250 .

Indice prevalenza $P M I=L Q_{i s}=\frac{E_{i s}}{E_{s}} / \frac{E_{i}}{E}$

dove $E_{i s}$ è il numero di addetti nelle unità locali nel sistema locale $s$ specializzato nel settore $i$ (HoReCa sector) con meno di 250 addetti; $E_{s}$ è il numero di addetti nelle unità locali nel sistema locale $s$ con meno di 250 addetti; $E_{i}$ è il numero di addetti in Italia specializzati nel settore $i(\mathrm{HoReCa})$ con meno di 250 addetti; ed $E$ indica l'occupazione totale in Italia con meno di 250 addetti.

Il secondo quoziente indica la maggiore presenza nella specializzazione precedente delle piccole e medie imprese sulla media nazionale. Precisamente, per i distretti industriali sono stati calcolati quattro indici, ma in questa prima approssimazione non applicheremo gli indici relativi all'industria principale, dato che analizziamo solo il comparto turistico, limitatamente alla voce $\mathrm{H}^{57}$.

\footnotetext{
57 I due rapporti di concentrazione possono essere calcolati sia per numero di addetti che per numero di imprese. Procederemo ad analizzare la forza lavoro invece che l'imprenditorialità, nonostante i risultati siano diversi risultano molto simili e ovviamente strettamente collegati.
} 
Si è individuato i sistemi locali del lavoro che registrano un'alta concentrazione dell'occupazione nel comparto turistico. Per quanto riguarda le modalità di rappresentazione dei valori dei coefficienti di localizzazione si è preferito adottare uno schema fisso secondo classi predefinite, al fine di permettere un'immediata interpretazione dei risultati ed un significativo confronto nell'ambito di una stessa area tematica come rappresentato da altri autori (Menghinello, 2002). In particolare, le classi di valore sono così definite: [0-1], [1-1.5], [1.5-3] e [>3 $]^{58}$. Queste classi contraddistinguono ben definiti livelli di concentrazione locale del fenomeno in esame: ad esempio la classe [1-1.5] identifica livelli di concentrazione relativa compresi tra la media nazionale e valori superiori del $50 \%$. Evidenziamo i risultati dell'analisi nella mappa sottostante. L'indice raffigurato è il tasso di specializzazione turistica (figura 2.6).

Il coefficiente di localizzazione consente di misurare l'intensità di un fenomeno a prescindere dalla sua rilevanza assoluta. D'altro canto, l'assenza di qualsiasi riferimento dimensionale può portare alla sopravvalutazione dell'importanza del fenomeno a livello locale. Al fine di evitare questo inconveniente si è posto una soglia minima in termini di contributo di ciascun sistema locale all'industria turistica nazionale (settore $\mathrm{HoReCa}$ ). Tale soglia, pari allo $0,01 \%$ esclude $i$ casi in cui la dimensione sia uno dei fattori maggiori relativi alla specializzazione, senza compromettere l'analisi delle realtà locali emergenti. La stessa soglia è utilizzata, per esempio, in Menghinello come contributo minimo alle esportazioni nazionali di un sistema locale (ibidem, 2002).

\subsubsection{I risultati del primo studio}

I risultati dell'analisi relativi al primo indice sono raffigurati nella figura 2.6. Il secondo indice di specializzazione (Piccola Impresa) non è presentato nella mappa perché varia tra la media nazionale e 1,05. Questo significa che non ci sono SLL che registrano una percentuale di piccole e medie imprese nel settore $\mathrm{HoReCa}$ minore del 95\% in media. Questo in parte conferma le caratteristiche del settore turistico principalmente basato su un tessuto di micro e piccole imprese.

${ }^{58} \mathrm{La}$ parentesi tonda o quadrata indica rispettivamente, estremi esclusi o inclusi per l'intervallo considerato. 
Figura 2.6: Il "settore" HoReCa in Italia;

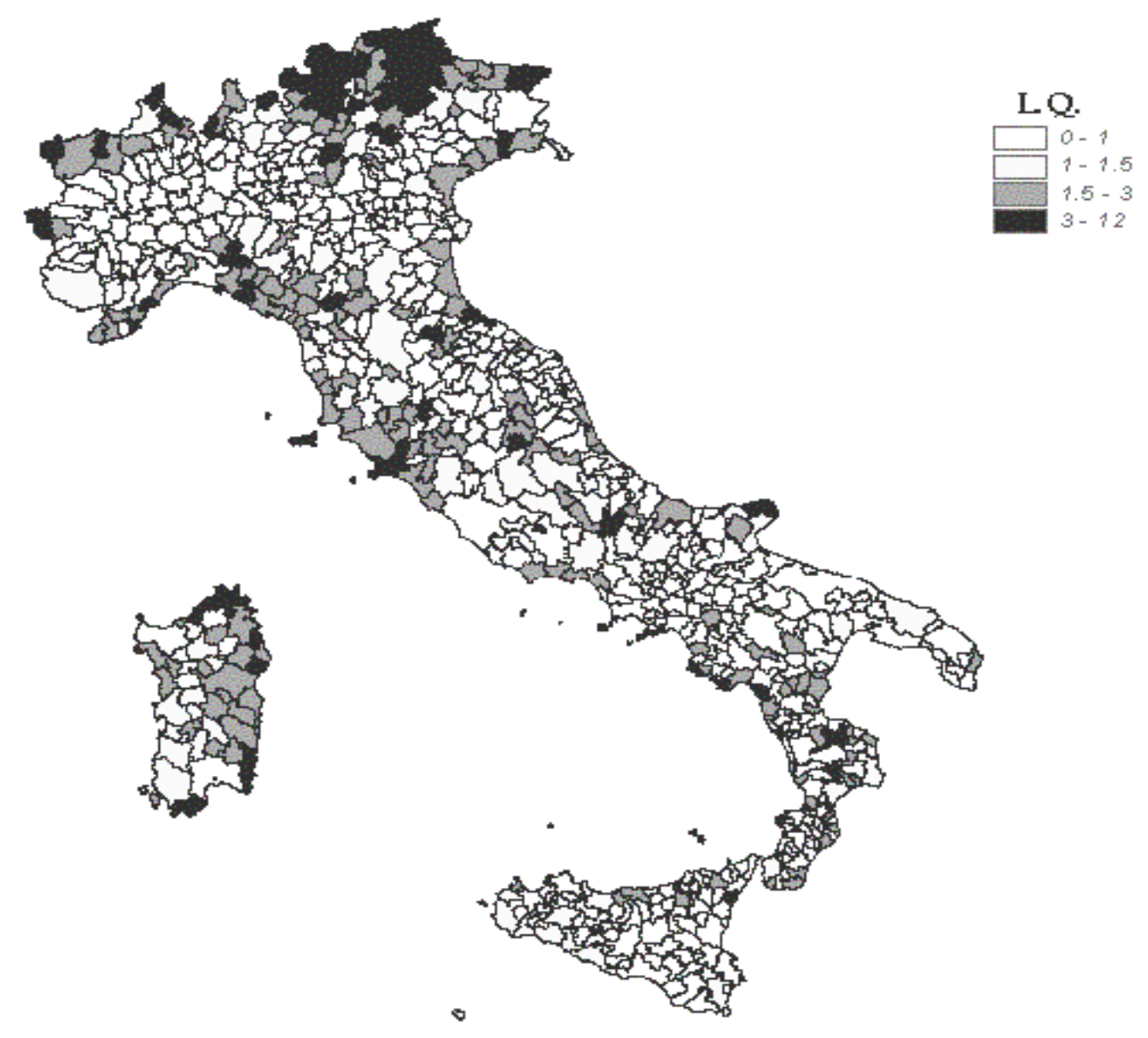

Fonte: nostra elaborazione su dati Censimento ISTAT, 1996;

Il primo indice di specializzazione varia tra la media nazionale e 12 (12 volte maggiore della media nazionale individuati nei SLT più scuri). Sono risultati circa 300 sistemi locali turistici (SLT) in tutta Italia con una maggiore specializzazione nel settore $\mathrm{HoReCa}$ in confronto alla media nazionale. I 300 SLT hanno in media 8 addetti e sono principalmente caratterizzati da piccole imprese, sono eterogenei e riflettono destinazioni turistiche relative diversi segmenti turistici. Infine sono diffusi in tutta Italia, anche se il nord Italia risulta avere un indice di localizzazione più alto. Le coste registrano un alto tasso di specializzazione, ma i valori più alti sono registrati nelle località montane (Trentino). A livello amministrativo le regioni con livelli più alti risultano il Trentino, la Toscana, la Liguria, l'Emilia Romagna ed il Veneto e la Sardegna.

Le città d'arte hanno valori di specializzazione superiori alla media nazionale, ma non risultano tra i SLT maggiormente specializzati. Per esempio, Firenze, Roma e Venezia, hanno indici di localizzazione prossimi a 2. Questo perché tale indice riconosce una forte specializzazione in una sola attività, quindi nei SLT diversificati questa specializzazione pesa 
in misura minore. Inoltre, $\mathrm{i}$ miglior $25 \mathrm{SLT}^{59}$ hanno un percentuale di addetti nella voce $\mathrm{H}$ attorno al 41\%, con picchi di 60\% nelle stazioni sciistiche (Ad esempio, Canazei e Bardonecchia).

I SLT analizzati appartengono a segmenti turistici eterogenei. Alcuni come Canazei, Badia e Cortina sono tipicamente del turismo da neve ed invernale, altri come Amalfi, Diano Marina, Porto Azzurro e L'Elba appartengono al turismo di mare e prevalentemente estivo. Altri ancora risultano misti, come Limone sul Garda o Fiuggi. Si nota inoltre una certa prevalenza di stazioni sciistiche, circa il 45\% dei sistemi locali analizzati.

Analizzando la presenza della piccola impresa, l'ipotesi che i sistemi locali turistici seguissero in certa misura la caratteristica dei distretti industriali, cioè registrassero una prevalenza di piccole e medie imprese, è rafforzata ampiamente.

Circa il 90\% degli addetti nei sistemi locali analizzati lavorano in piccole e medie imprese che registrano meno di 20 dipendenti. Tale misura, che risulta comunque abbastanza grande per evidenziare l'eventuale presenza di imprese familiari e artigiane, viene ancora di più rafforzata quando si analizza la percentuale di imprese con meno di 5 dipendenti. La media dei sistemi locali analizzati staziona addirittura intorno al 75\%, con medie intorno al 90\% di Porto Azzurro e Limone sul Garda. Questo sottolinea ancora di più come il comparto turistico sia basato prevalentemente su piccole imprese.

In questo modo si rileva come un sistema locale turistico sia caratterizzato da agglomerazioni di piccole imprese e come le lenti distrettuali siano utili ed efficienti per studiare questo tipo di modello di sviluppo locale. A conferma si evidenzia come la media di addetti nelle imprese raggiunga in maniera scarsa le cinque unità con picchi minimi di 3 unità a Malcesine e a Porto Azzurro, picchi massimi di oltre 7 a Cortina d'Ampezzo, Limone sul Garda e Pinzolo.

Innanzitutto, i risultati di questa analisi sottolineano come la metodologia di analisi tramite SLL sia applicabile all'industria turistica al fine della individuazione dei SLT come descritti nella prima parte di questo scritto. I SLT sono fortemente specializzati nell'industria turistica come la loro "core industry", le unità locali sono localizzate all'interno dei confini dei SLL.

Riteniamo tuttavia utile soffermarci su due problematiche. La prima è la definizione di turismo utilizzata che anche se può avvicinarsi molto all'idea di turismo potrebbe non registrare dei sistemi locali specializzati in altre parti della filiera; a tal fine è necessario provvedere alla costruzione di una filiera turistica ad hoc su cui calcolare gli indici di localizzazione. In seconda istanza riteniamo opportuno aggiornare anche il database utilizzato in base al Censimento ISTAT del 2001. Cercheremo di affrontare questi aspetti nel prossimo paragrafo.

59 Alassio, Amalfi, Badia, Bardonecchia, Bormio, Campo Nell'elba, Canazei, Castelrotto, Cortina d'ampezzo, Diano Marina, Fiera Di Primiero, Fiuggi, Forio, Limone Sul Garda, Malcesine, Moena, Morgex, Nova Ponente, Ortisei, Peio, Pinzolo, Porto Azzurro, Positano, Saint-Vincent, San Candido. 


\subsection{SECONDO STUdiO: IL CENSIMENTO DEL 2001 ED LA FILIERA}

\section{TURISTICA}

In questo paragrafo proseguiremo l'analisi iniziata nel capitolo che ci ha portato ad una prima approssimazione all'individuazione nel territorio italiano dei sistemi locali del lavoro specializzati, in maniera esclusiva e non, nell'industria turistica (settore HoReCa). Nel paragrafo precedente infatti sono stati applicati gli indici di localizzazione turistica al settore dei Ristoranti, Hotel e Caffè rappresentato dalla voce $\mathrm{H}$ del censimento intermedio ISTAT dellindustria e dei servizi del 1996. Tale analisi ha permesso di descrivere un primo panorama dei SLT italiani, ma il procedimento utilizzato ci porta a dover risolvere due tipi di problematiche. La prima riguarda appunto la definizione di turismo utilizzata, che anche se può approssimarsi a quella di attività turistiche (OECD, 1999) non fornisce una stima reale sull'incidenza di questa attività nel turismo. Quindi si potrebbero tralasciare alcuni sistemi locali specializzati in altre aree della filiera turistica (trasporti e attività ricreative, per esempio). Il secondo problema riguarda il riferimento temporale dei dati che si riferiscono a circa 8 anni fa: il 1996. In questo paragrafo aggiorneremo la fonte dei dati utilizzando il censimento del 2001 e analizzeremo l'evoluzione temporale dei SLT. Infine, cercheremo di costruire una filiera ad hoc dell'industria turistica rifacendosi a contributi istituzionali come ISTAT, Commissione Europea e OECD.

Il nuovo censimento dell'ISTAT dell'anno 2001 è disponibile on-line sul sito ISTAT da marzo 2004. Il ritardo nella disponibilità dei dati e la poca funzionalità del datawarehouse nei primi mesi di accessibilità ha reso ancora più difficile un'analisi su una sostanziale mole di dati di questo tipo.

È inoltre necessario aprire una parentesi sull'unità territoriale utilizzata ${ }^{60}:$ i sistemi locali del lavoro. I confini territoriali presi in considerazione fanno riferimento alla realtà amministrativa del 1991, cioè ai comuni esistenti a tale data. Nel periodo 1991-2001 sono state istituite 8 nuove province, mentre altre 8 hanno ceduto comuni alle nuove (si tratta delle nuove province di Biella, Verbano-Cusio-Ossola, Lecco, Lodi, Prato, Rimini, Vibo Valentia e Crotone, istituite nel 1992 con comuni staccati dalle province di Vercelli, Novara, Como, Milano, Bergamo, Forlì-Cesena, Firenze e Catanzaro). I dati del 2001 sono stati, quindi, ricalcolati ai confini del 1991 per effettuare un confronto tra dati omogenei. Inoltre altre modifiche territoriali ${ }^{61}$ sono state effettuate successivamente e sono state riportate ai comuni del 1991.

${ }^{60}$ Come già detto, nonostante il lasso di tempo trascorso, pensiamo che i sistemi locali del 1991 siano idonei a questo tipo di analisi. Prima di tutto, perché, pensiamo che la vita sociale e le relazioni socioeconomiche di un sistema locale, siano delle caratteristiche che registrano una certa continuità nel tempo. Inoltre, la possibilità di utilizzare dati georeferenziati da parte dell'ISTAT è valida al momento solo per i sistemi locali costruiti con il censimento del 1991. I nuovi SLL per il censimento del 2001 saranno disponibili solo a fine 2004-inizio 2005.

${ }^{61}$ Nello stesso periodo sono stati istituti 10 nuovi comuni: Mosso (BI), Montiglio Monferrato (AT), Cavallino Treporti (VE), Due Carrare (PD), Porto Viro (RO), Fiumicino (RM), Statte (TA), Padru (SS) e Monserrato (CA) ed infine il 15 ottobre 2001 è stato istituito il comune di Fonte Nuova (RM), i cui dati sono disponibili nel censimento 2001. Nello stesso periodo sono stati soppressi 9 comuni: Mosso Santa Maria (BI), Pistolesa (BI), Colcavagno (AT), Montiglio (AT), Scandeluzza (AT), Carrara San Giorgio (PD), Carrara Santo Stefano (PD), Contarina (RO) e Donada (RO). Sono cambiati i confini di 28 comuni (esclusi i comuni che nel periodo 1991-2001 hanno aggregato o staccato zone di territorio disabitate); si tratta dei comuni di Prascorsano (TO), Valperga (TO), Cerreto d'Asti (AT), Piovà Massaia (AT), Casal Cermelli (AL), Castellazzo Bormida (AL), Ardenno (SO), Forcola (SO), Arluno (MI), Milano (MI), Santo Stefano Ticino (MI), Sesto San Giovanni (MI), Cazzago San Martino (BS), Ospitaletto (BS), Passirano (BS), Rovato (BS), Venezia (VE), Cesena (FC), 
Infine, per quanto riguarda i codici di censimento (Ateco 1991-2002), varie voci sono cambiate, ma il sito dell'ISTAT permette di effettuare le analisi in base ai codici base 1991 e rende disponibili direttamente i dati omogenei per codici Ateco 2001. Per questo motivo e per utilizzare un riferimento temporale maggiormente ampio utilizzeremo come base di riferimento per analizzare l'evoluzione dei sistemi locali turistici la crescita tra l'anno 1991-2001. Questo ci permetterà di focalizzare l'analisi su 10 anni di evoluzione.

Il primo problema è quello della costruzione della filiera allargata del turismo. Il riferimento principale è l'Appendice E della classificazione Ateco91 "Attività collegata al turismo" in tabella 2.4. Tutte queste attività classificate fino a tre codici Ateco sono considerate dall'ISTAT come parte integrante delle attività turistiche di un luogo. D'altra parte anche la OECD (1999) ha analizzato in alcuni contributi la forza lavoro del turismo focalizzando la propria analisi quantitativa a livello di ISIC two digits includendo le stesse classi dell'Appendice E. Anche se non tutte le attività in queste industrie possono essere attribuite al turismo, non essendo stati calcolati pesi o percentuali per analizzare solo gli addetti relazionate al turismo, si è deciso di utilizzare le tre categorie ${ }^{62}(55,60-63,92)$ al $100 \%$ dato che sono innegabilmente coinvolte ${ }^{63}$ (OECD, 1999).

Gambettola (FC), Guidonia Montecelio (RM), Mentana (RM), Roma (RM), Castelforte (LT), Santi Cosma e Damiano (LT), Roccaforzata (TA), Taranto (TA), Buddusò (SS) e Cagliari (CA).

6255 : Hotel, resterauntes and cafes; 60-63: Trasport and travel; 92: recreational, cultural and sporting activities

${ }^{63}$ Un altro procedimento utilizzato è stato quello di costruire una filiera suddivisa per due caratteristiche: se direttamente o indirettamente destinate alle attività turistiche. Le attività all'interno della filiera sono state divise in: a) dirette ed indirette e quindi b) indirette complementari, c) indirette competitive con la comunità di residenti. In altre parole se 100\% dedicate ai turisti o se dedicate anche ai residenti. Per esempio, per quanto riguarda gli alberghi questa è un'attività in cui gli addetti sono dedicati esclusivamente ai turisti, mentre, per quanto riguarda i trasporti c'è una parte della forza lavoro che è dedicata dalla comunità locale (i residenti) ed un'altra ai turisti. Questa analisi pone tuttavia dei problemi per calcolare che quota di ogni attività imputare. Si è cercato di relativizzare questo valore in base ad un indice costruito da: presenze turistiche/(residenti + presenze turistiche) o con: ricettività turistica/(ricettività turistica + residenti). Ad ogni modo si pensa che questo calcola sia poco preciso perché non prenda in considerazione la stagionalità dell'offerta e il tasso di utilizzo delle strutture dell'offerta. Per queste motivazioni si è preferito imputare al $100 \%$ tutte le attività. 
Tabella 2.4: Attività collegate al turismo :Appendice E - ATECO 91

\begin{tabular}{|c|c|}
\hline $\begin{array}{l}\text { 60. TRASPORTI TERRESTRI } \\
\text { 60.1- Trasporti ferroviari } \\
\text { 60.10.1- Trasporti ferroviari } \\
\text { 60.10.2- Servizi ausiliari delle ferrovie } \\
\text { 60.2- Altri trasporti terrestri } \\
\text { 60.21- Altri trasporti terrestri, regolari, di passeggeri } \\
\text { 60.22- Trasporti con taxi } \\
\text { 60.23- Altri trasporti su strada, non regolari, di passeggeri } \\
\text { 60.24- Altri trasporti terrestri di passeggeri } \\
\text { 61. TRASPORTI MARITTIMI E PER VIE D'ACQUA } \\
\text { 61.1- Trasporti marittimi e costieri } \\
\text { 61.11- Trasporti marittimi } \\
\text { 61.12- Trasporti costieri } \\
\text { 61.2- Trasporti per vie d'acqua interne (compresi i trasporti } \\
\text { lagunari) } \\
\text { 62. TRASPORTI AEREI } \\
\text { 62.1- Trasporti aerei di linea } \\
\text { 62.2- Trasporti aerei non di linea } \\
\text { 55. ALBERGHI } \\
\text { 55.1- Alberghi } \\
\text { 55.11- Alberghi e motel, con ristorante } \\
\text { 55.12- Alberghi e motel, senza ristorante } \\
\text { 55.2- Campeggi ed altri alloggi per brevi soggiorni } \\
\text { 55.21- Ostelli della gioventù e rifugi di montagna } \\
\text { 55.21.1- Ostelli della gioventù } \\
\text { 55.21.2- Rifugi di montagna } \\
\text { 55.22- Campeggi e aree attrezzate } \\
\text { 55.23- Altri tipi di alloggio n.c.a. } \\
\text { 55.23.1- Villaggi turistici } \\
\text { 55.23.2- Colonie, case per ferie e case di riposo (senza cure } \\
\text { mediche) } \\
\text { 55.23.3- Gestione di vagoni letto } \\
\text { 55.23.4- Affittacamere per brevi soggiorni, case per vacanze } \\
\text { 55.23.5- Agriturismo } \\
\text { 55.23.6- Altri esercizi alberghieri complementari (compresi i } \\
\text { residence) } \\
\text { 55.3- Ristoranti } \\
\text { 55.30.1- Ristoranti, trattorie, pizzerie, osterie e birrerie con cucina } \\
\text { 55.30.2- Rosticcerie, friggitorie, pizzerie a taglio con } \\
\text { somministrazione } \\
\text { 55.30.3- Gestione di vagoni ristoranti } \\
\text { 55.30.4- Servizi di ristorazione in self-service } \\
\text { 55.30.5- Ristoranti con annesso intrattenimento e spettacolo } \\
\text { 55.4- Bar } \\
\text { 55.40.1- Bar e Caffè } \\
\text { 55.40.2- Gelaterie } \\
\text { 55.40.3- Bottiglierie ed enoteche con somministrazione } \\
\text { 55.40.4- Bar, caffè con intrattenimento e spettacolo } \\
\text { 55.5- Mense e forniture di pasti preparati } \\
\text { 55.51- Mense } \\
\text { 55.52- Forniture di pasti preparati } \\
\text { (n) }\end{array}$ & $\begin{array}{l}\text { 92.3 ATTIVITA' RICREATIVE E CULTURALI } \\
\text { 92.33- Attività riguardanti i parchi di divertimento } \\
\text { 92.34- Altre attività di intrattenimento e di spettacolo } \\
\text { n.c.a. } \\
\text { 92.34.1- Discoteche, sale da ballo, night club e simili } \\
\text { 92.34.2- Sale giochi e biliardi } \\
\text { 92.34.3- Circhi ed altre attività itineranti di intrattenimento e } \\
\text { di spettacolo } \\
\text { 92.34.4- Altre attività di intrattenimento e spettacolo (parchi } \\
\text { di attrazione e simili) } \\
\text { 92.5- Attività di biblioteche, archivi, musei ed altre } \\
\text { attività culturali } \\
\text { 92.51- Attività di biblioteche e archivi } \\
\text { 92.52- Attività dei musei e conservazione dei luoghi e } \\
\text { monumenti storici } \\
\text { 92.53- Attività di orti botanici, giardini zoologici e riserve } \\
\text { naturali } \\
\text { 92.6- Attività sportive } \\
\text { 92.61- Gestione di stadi e altri impianti sportivi } \\
\text { 92.61.1- Gestione di stadi } \\
\text { 92.61.2- Gestione di piscine } \\
\text { 92.61.3- Gestione di campi da tennis } \\
\text { 92.61.4- Gestione di impianti polivalenti } \\
\text { 92.61.5- Gestione di altri impianti sportivi } \\
\text { 92.61.6- Gestione di palestre } \\
\text { 92.62- Altre attività sportive } \\
\text { 92.62.1- Enti e organizzazioni sportive } \\
\text { 92.62.2- Attività professionali sportive indipendenti } \\
\text { 92.62.3- Attività di organizzazione e promozione di eventi } \\
\text { sportivi } \\
\text { 92.7- Altre attività ricreative } \\
\text { 92.71- Attività riguardanti il gioco d'azzardo } \\
\text { 92.72- Atre attività ricreative n.c.a. } \\
\text { 92.72.1- Stabilimenti balneari (marittimi, lacuali e fluviali) } \\
\text { 92.72.2- Altre attività ricreative n.c.a. } \\
\text { 63. ATTIVITA' DELLE AGENZIE DI VIAGGIO } \\
\text { 63.3- Attività delle agenzie di viaggio e degli operatori } \\
\text { turistici } \\
\text { 60.30.1- Attività delle ag. di viaggio e turismo (compresi i } \\
\text { tour operator) } \\
\text { 60.30.2- Attività di guide e accompagnatori turistici }\end{array}$ \\
\hline
\end{tabular}

Fonte: ISTAT-ATECO91; 
Procediamo quindi ad integrare le attività dell'appendice E con quelle che possono far parte della filiera turistica di un luogo. Al fine di costruire una filiera allargata partiamo da un'ipotesi di un processo di "fornitura di servizi turistici". Nella figura 2.7 è presentato un modello del settore turistico proposto nel rapporto sul turismo della Commissione Europea del 2003. Il modello descrive nella colonna verticale la filiera integrata del turismo costituita dai trasporti, attrazioni (culturali, ricreative, etc.), strutture ricettive, catering e ristoranti. Nel quadrato centrale sono presentati gli intermediari legati alla filiera turistica. Nella parte orizzontale si presentano tutte quelle attività di integrazione orizzontale costituite da tutti i produttori, distributori ed intermediari di attività non strettamente turistiche.

Figura 2.7: A tourism sector model;
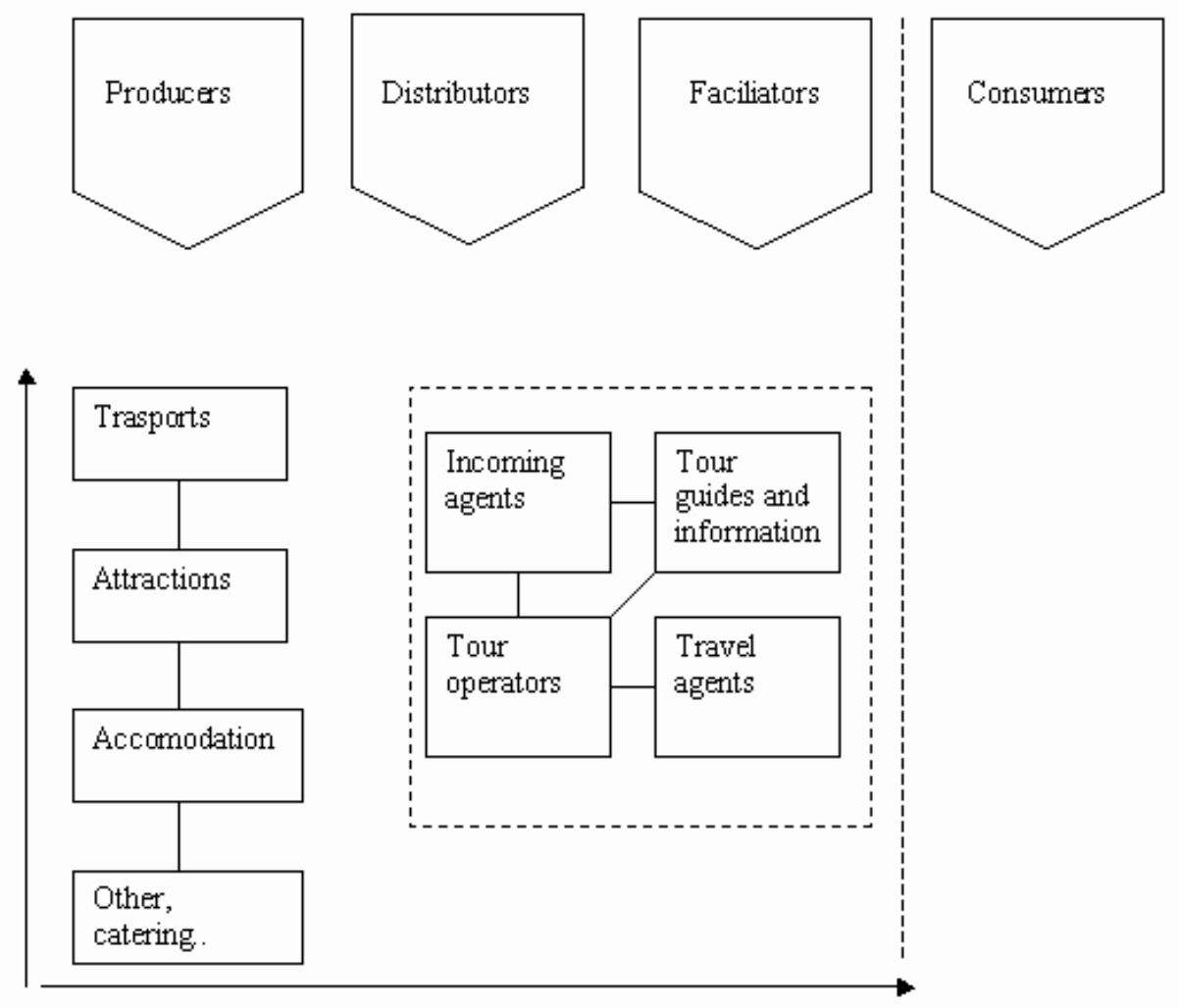

Horizontal integrationand consolidation

Fonte: Commissione European, 2003; adattato da Poon, 1993 e Coopers et al. 1998.

Si propone quindi una filiera turistica basata sul core dell'analisi della Commissione Europea basata principalmente sulla filiera verticale integrata (Alloggiamento, trasporti, attività ricreative, ristorazione) che risulta molto simile alla Appendice E dell'ISTAT presentata nelle pagine precedenti. Si aggiungono le categorie degli intermediari come agenzie di viaggi e tour operator. Infine, al fine di catturare i legami con il settore della manifattura e dell'agricoltura, integriamo le voci descritte con una voce che denominiamo Artigianato artistico $(26.1,26.2)$ con la voce Agricoltura (01.13) e con la locazione di mezzi di trasporto e case di proprietà $(70.2,71.1)$. Presentiamo la filiera allargata nella tabella 2.4. 
Tabella 2.4. Filiera allargata dell'industria turistica ${ }^{64}$;

\begin{tabular}{|c|c|}
\hline Agricoltura & Attività Ricreative, Culturali e Sportive \\
\hline $\begin{array}{l}\text { 1.13 Coltivazione di frutta, frutta a guscio, prodotti } \\
\text { destinati alla preparazione di bevande, spezie }\end{array}$ & $\begin{array}{l}92.3 \text { Altre attività dello spettacolo, di } \\
\text { intrattenimento e divertimento }\end{array}$ \\
\hline Artigianato Artistico & $\begin{array}{l}92.5 \text { Attività di biblioteche, archivi, musei ed } \\
\text { altre attività culturali }\end{array}$ \\
\hline $\begin{array}{l}26.1 \text { Fabbricazione di vetro e di prodotti in vetro } \\
\text { (artigiani) }\end{array}$ & $\begin{array}{l}\text { 92.6 Attività sportive } \\
92.7 \text { Attività ricreative }\end{array}$ \\
\hline $\begin{array}{l}26.2 \text { Fabbricazione di prodotti ceramici non } \\
\text { refrattari, non destinati all'edilizia e di prodotti } \\
\text { ceramici refrattari (artigiani) }\end{array}$ & $\begin{array}{l}\text { Trasporti di persone } \\
\text { 60.1 Trasporti ferroviari } \\
\text { 60.2 Altri trasporti terrestri }\end{array}$ \\
\hline Alberghi e Ristoranti & 61.1 Trasporti marittimi e costieri \\
\hline 55.1 Alberghi & 61.2 Trasporti per vie d'acqua interne \\
\hline 55.2 Campeggi ed altri alloggi per brevi soggiorni & (compresi i trasporti lagunari) \\
\hline 55.3 Ristoranti & 62.1 Trasporti aerei di linea \\
\hline $55.4 \mathrm{~B}$ & Agenzie Di Viaggio \\
\hline $\begin{array}{l}\text { Noleggio e Affitto } \\
70.2 \text { Locazione di beni immobili }\end{array}$ & $\begin{array}{l}\text { 63.3 Attività delle agenzie di viaggio e degli } \\
\text { operatori turistici; attività di assistenza turistica }\end{array}$ \\
\hline
\end{tabular}

Fonte: nostra elaborazione su Ateco2002;

A nostro avviso, ci aspettiamo che esista una specializzazione, in una parte od in tutta la filiera presentata, nei sistemi locali turistici e che questa proxi sia utilizzabile per identificare sul territorio italiano i SLT in base al censimento 2001. Quindi in base alle motivazioni metodologiche presentate nella premessa di questo capitolo applichiamo ai SLL un indice di concentrazione.

Presentiamo l'indice di concentrazione nella forma usuale:

$$
L Q_{i s}=\frac{E_{i s}}{E_{s}} / \frac{E_{i}}{E}
$$

Dove $E_{i s}$ è il numero di addetti nelle unità locali nel sistema locale $s$ specializzato nel attività $i$ (della filiera allargata); $E_{s}$ è il numero di addetti nelle unità locali nel sistema locale $s ; E_{i}$ è il numero di addetti in Italia specializzati nell'attività $i$ (della filiera allargata); e $E$ è l'occupazione totale in Italia.

Per quanto riguarda le modalità di rappresentazione dei coefficienti di localizzazione si continua ad adottare uno schema fisso secondo classi predefinite, al fine di permettere un'immediata interpretazione dei risultati. L'indice raffigurato è il tasso di specializzazione turistica. Inoltre, come già nel primo studio si è posto una soglia minima in termini di contributo di ciascun sistema locale all'industria turistica nazionale. Tale soglia, pari allo $0,01 \%$, esclude $i$ casi in cui la dimensione sia uno dei fattori maggiori relativa alla specializzazione, senza compromettere l'analisi delle realtà locali emergenti.

Seguendo l'approccio di Sforzi (1997a) presentato nel primo capitolo, analizziamo i 784 sistemi locali del lavoro selezionando quelli specializzati rispettivamente in: (1) industria (2) servizi, e (3) in servizi alle imprese, (4) servizi ai consumatori e finalmente

\footnotetext{
${ }^{64}$ Per il contenuto esteso di tutte le voci si rimanda all'Appendice con le voci estese della filiera.
} 
specializzati nella (5) filiera turistica allargata. I dati sono stati collezionati in base al censimento ISTAT 2001 per ogni comune e poi aggregato in base ai SLL del 1991 ${ }^{65}$. La mappa sottostante raffigura quei SLL che registrano un indice di localizzazione superiore a 1. La mappa rappresenta circa 300 sistemi locali turistici con un LQ che va dalla media nazionale fino ad 8 . I valori più alti sono concentrati nel nord Italia (Trentino e Alto Adige) e nel centro Italia (Liguria, Toscana e Lazio). La mappa è simile a quella costruita in base al settore $\mathrm{HoReCa}_{e} 6$ e registra anche le città d'arte come Firenze, Roma e Venezia, località estive, invernali e destinazioni lacustri.

Figura 2.8: I sistemi locali del lavoro in base alla filiera turistica della tabella 2.4;

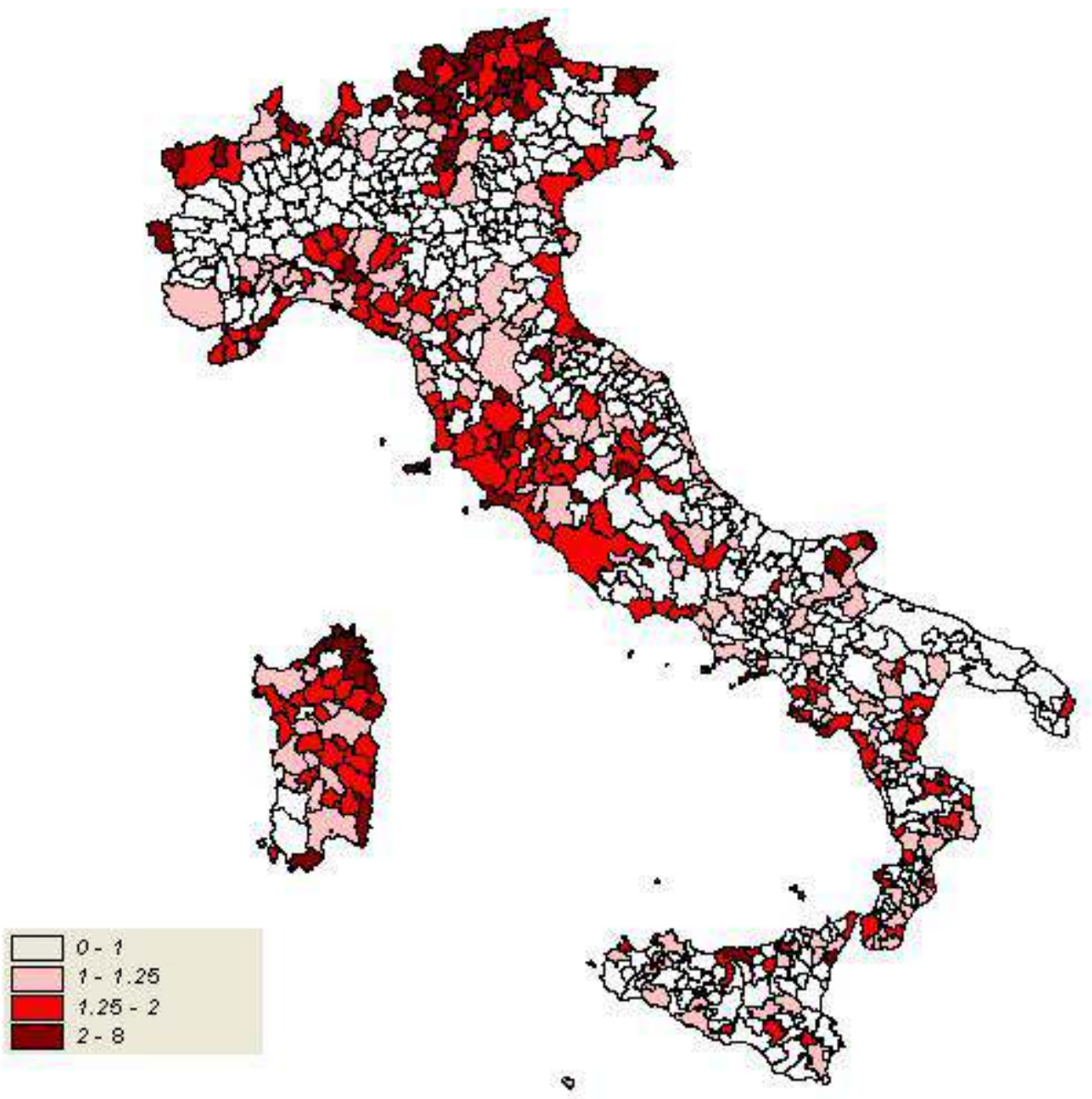

Fonte: nostra elaborazione su dati Istat, 2001;

Analizziamo nelle prossime pagine le varie configurazioni della filiera allargata. Prima di tutto al fine di evidenziare quei SLT che hanno legami con altri settori presentiamo nella figura 2.9 i SLT con un indice di concentrazione maggiore di 1 suddivisi per specializzazione in altre attività come in Sforzi (1997b): 1) non specializzati in attività turistiche, 2) specializzate nel turismo e nell'industria, 3) specializzati nel turismo, servizi e commercio, 4) diversificati.

${ }^{65}$ La mappe relativamente alle elaborazioni 1, 2, 3, 4 sono disponibili in appendice. 
Figura 2.9: Sistemi Locali Turistici per classi di specializzazione;

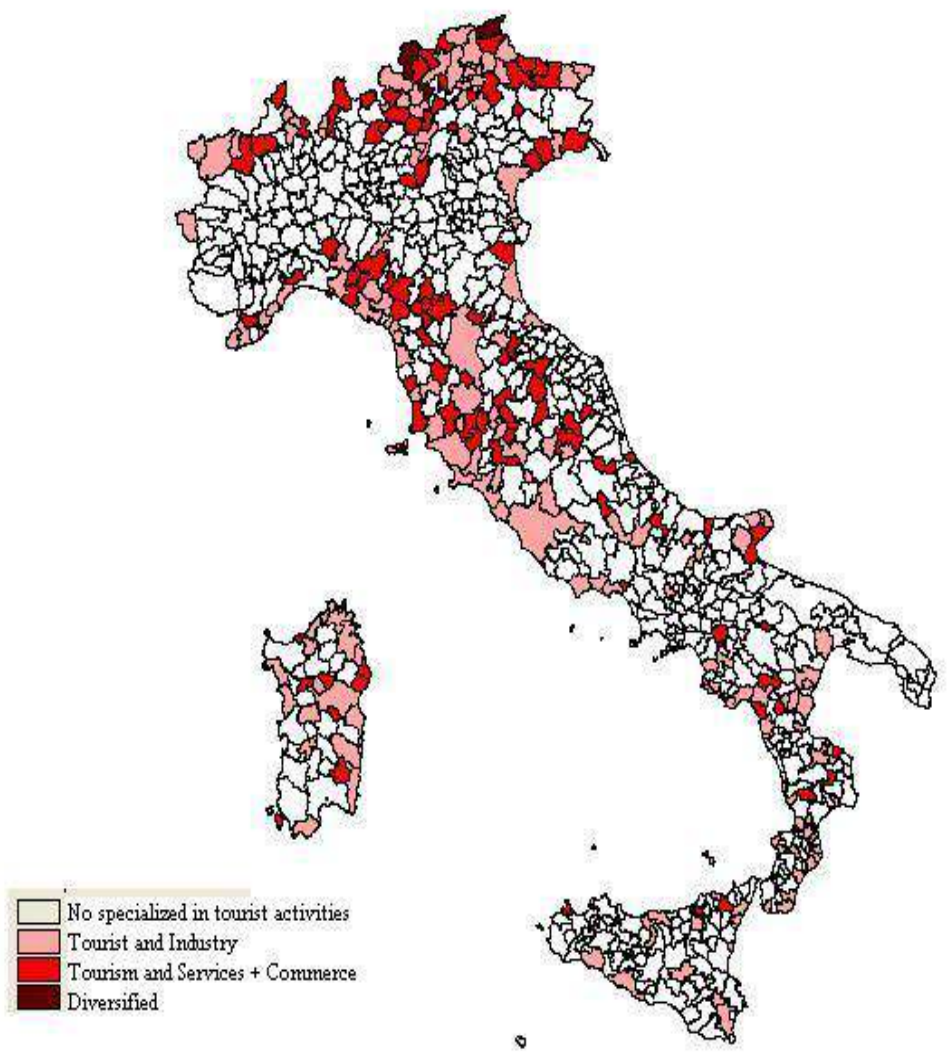

Fonte: nostra elaborazione su dati Istat, 2001;

Al fine di effettuare una comparazione tra il censimento del 1996 e quello del 2001, ed al fine di testare la metodologia del primo studio, replichiamo la proxi utilizzata nel paragrafo precedente. Presentiamo quindi la mappa dei SLL specializzati nel settore HoReCa ma in base al censimento 2001. I risultati degli anni 1996-2001 sono simili e presentano gli stessi patterns territoriali. Si denota, come la proxi $\mathrm{HoReCa}$ registra molti più sistemi locali turistici che la filiera allargata, nonostante questa ultima sia stata costruita in senso esteso. A nostro avviso, uno dei problemi che si incontrano nell'utilizzare il settore HoReCa come proxi di identificazione è che i medie-grandi centri pesano mediamente di più, perché hanno una specializzazione in queste attività per i cittadini maggiore che per il turismo. 
Figura 2.10: Sistemi Locali del lavoro settore HoReCa (Voce H) su dati 2001;

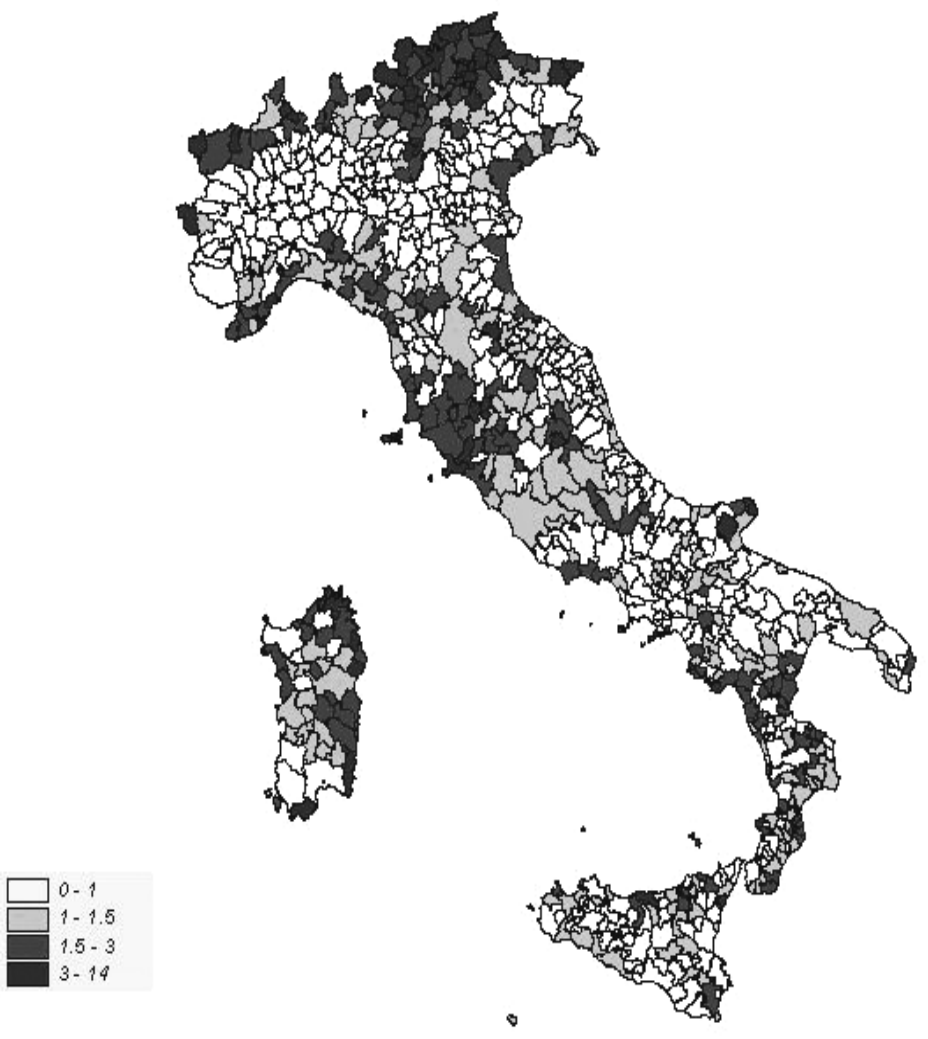

Fonte: nostra elaborazione su dati Istat, 2001;

Nella tabella 2.5 riassumiamo i risultati raggiunti in questo paragrafo, in particolare indichiamo i SLT con indici di concentrazioni più alti relativamente ad alloggiamento, agenzie di viaggio, pil procapite, spesa stranieri e peso dell'agricoltura. La tabella presenta i sistemi locali, già evidenziati nel primo studio in base al settore $\mathrm{HoReCa}$ e propone un primo panorama di destinazioni eterogenee turistiche nel territorio italiano. 
Tabella 2.5: SLL con indici di concentrazione più alti

\begin{tabular}{|c|c|c|c|c|c|}
\hline S11 & Lq alberghi & S11 & Lq campeggi & S11 & Lq agenzie di viaggio \\
\hline 109 & Limone sul garda & 141 & Badia & 345 & Portoferraio \\
\hline 475 & Positano & 530 & Vieste & 440 & Forio \\
\hline 440 & Forio & 344 & Porto azzurro & 673 & Terrasini \\
\hline 181 & Malcesine & 152 & Ortisei & 703 & Sciacca \\
\hline 691 & Taormina & 153 & San candido & 445 & Sorrento \\
\hline 414 & Fiuggi & 629 & Tropea & 570 & Policoro \\
\hline 459 & Amalfi & 765 & Muravera & 181 & Malcesine \\
\hline 441 & Ischia & 463 & Camerota & 109 & Limone sul garda \\
\hline 733 & Arzachena & 159 & Canazei & 745 & Santa teresa di gallura \\
\hline 151 & Nova ponente & 620 & Pizzo & 629 & Tropea \\
\hline 766 & Pula & 146 & Castelrotto & 441 & Ischia \\
\hline 146 & Castelrotto & 673 & Terrasini & 594 & Praia a mare \\
\hline 159 & Canazei & & & 740 & Olbia \\
\hline 438 & Capri & & & 691 & Taormina \\
\hline S11 & Pil pro-capite & S11 & Spesa stranieri & S11 & Lq agricoltura \\
\hline 159 & Canazei & 403 & Roma & 491 & Isola del gran sasso \\
\hline 141 & Badia & 337 & Firenze & 360 & Gaiole in chianti \\
\hline 142 & Bolzano & 184 & valpolicella & 361 & Montalcino \\
\hline 197 & Cortina d'ampezzo & 224 & Latisana & 380 & Gubbio \\
\hline 144 & Brunico & 212 & Venezia & 390 & Acquapendente \\
\hline 152 & Ortisei & 211 & San dona' di piave & 364 & San quirico d'orcia \\
\hline 345 & Portoferraio & 278 & Rimini & 703 & Sciacca \\
\hline 169 & Pinzolo & 210 & Portogruaro & 224 & Latisana \\
\hline 109 & Limone sul garda & 445 & Sorrento & 255 & Pavullo nel frignano \\
\hline 195 & Agordo & 231 & Monfalcone & 475 & Positano \\
\hline 156 & Vipiteno & 69 & Sestri levante & 387 & Fabro \\
\hline 143 & Bressanone & 362 & Montepulciano & 335 & Castelfiorentino \\
\hline 227 & Tarvisio & 2 & Bardonecchia & 760 & Siniscola \\
\hline 149 & Merano & 181 & Malcesine & 388 & Orvieto \\
\hline 167 & Moena & 441 & Ischia & 372 & Pitigliano \\
\hline 272 & Cattolica & 55 & Diano marina & 440 & Forio \\
\hline
\end{tabular}

Fonte: nostra elaborazione su Censimento ISTAT 2001 e dati UIC 2002.

Dopo aver presentato un panorama della struttura dell'offerta nel 2001 dei SLT italiani, nel prossimo paragrafo cercheremo di proporre un panorama della domanda suddivisa per i 784 sistemi locali del lavoro, al fine di evidenziare eventuali similitudini con le mappe precedenti.

Concludiamo questo paragrafo con l'analisi dell'evoluzione dei SLT presentati tra il 1991 e il 2001. Nella figura 2.11 presentiamo i percentili di crescita occupazione dei SLL del 1991, costruiti come il rapporto tra gli addetti nella filiera allargata del 2001 su quelli del 1991. In altre parole, la mappa sottostante evidenzia come i SLT sono cresciuti nel decennio di riferimento, evidenziando i best performers e se l'indice di concentrazione è legato a tale crescita. 
Figura 2.11: La crescita dell'occupazione della filiera turistica nei SLL, 1991-2001;

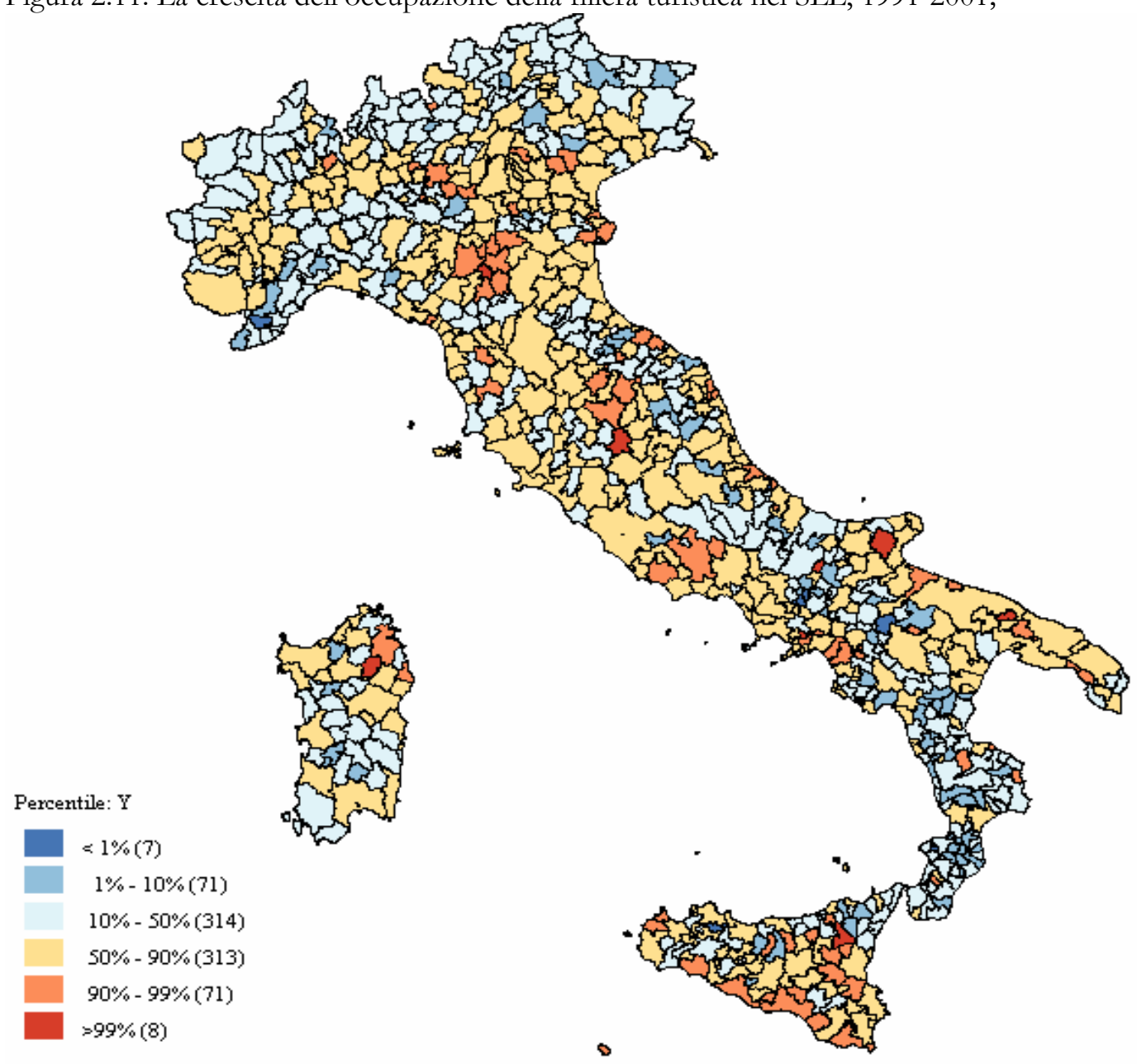

Fonte: nostra elaborazione su Censimento ISTAT 1991-2001.

Prima di tutto si nota, come la crescita (i SLL più scuri) non sia legata almeno visualmente all'indice di concentrazione. Questa mappa è molto diversa dalla mappa numero 2.8. Si vede, infatti, come le zone con più alti $L Q$, Trentino non registrino un tasso di crescita elevato. D'altro canto, invece, la crescita più diffusa avviene nei sistemi locali di Perugia, Bologna, e nella Sicilia meridionale. Inoltre, non si percepisce un trend particolare anche se il tasso sembra avere una certa diffusione spaziale, cioè i sistemi locali vicini hanno patterns di sviluppo simili. Approfondiremo questo aspetto nel quarto capitolo nell'analisi delle reti di sistemi locali. Vogliamo porre l'accento, infine, come la mappa sia costruita in base ad un tasso di crescita e quindi potrebbe sottostimare lo sviluppo di grandi aree che sono cresciute solo relativamente poco.

\subsubsection{Una breve analisi della domanda turistica}

In questo paragrafo, continuiamo l'elaborazione proponendo una breve analisi della domanda e analizzando se questa ultima segue patterns territoriali simili a quelli evidenziati nello studio dell'offerta.

Le unità di analisi territoriali sono ancora i sistemi locali del lavoro al fine sia di preparare un database ad hoc per le analisi del seguente capitolo sia per evidenziare tramite GIS i patterns territoriali simili tra la domanda ed offerta. La fonte utilizzata in questo 
paragrafo si riferisce principalmente alle statistiche sul turismo registrate a livello annuale dall'ISTAT (come presentate nella descrizione delle fonti di dati in questo capitolo) e le statistiche disponibili on-line dall'Unione Italiana Cambia (UIC), che registra la spesa degli italiani e degli stranieri nel territorio italiano e suddivise per residenza ${ }^{66}$. In particolare proponiamo come prima analisi in questo paragrafo l'analisi degli arrivi. Si ricorda che gli arrivi sono definiti come: "Il numero di clienti, italiani e stranieri, ospitati negli esercizi ricettivi (alberghieri o complementari) nel periodo considerato" (Istat, 2002)

Nella figura 2.12 si presentano i dati relativi agli arrivi totali suddivisi per sistema locali in Italia nell'anno 2001. La mappa presenta le destinazioni turistiche principali del panorama italiano. Le classi sono presentate tramite quartili al fine di una più semplice interpretazione ${ }^{67}$. Le città d'arte come Firenze, Venezia e Roma svolgono un ruolo preminente e la costa ligure-toscana e parte di quella romagnola registrano alti tassi di arrivi. Il sud Italia registra bassi livelli tranne per alcune zone della Puglia e della Sicilia ${ }^{68}$.

Figura 2.12: Arrivi totali (quartili), Italia 2001;

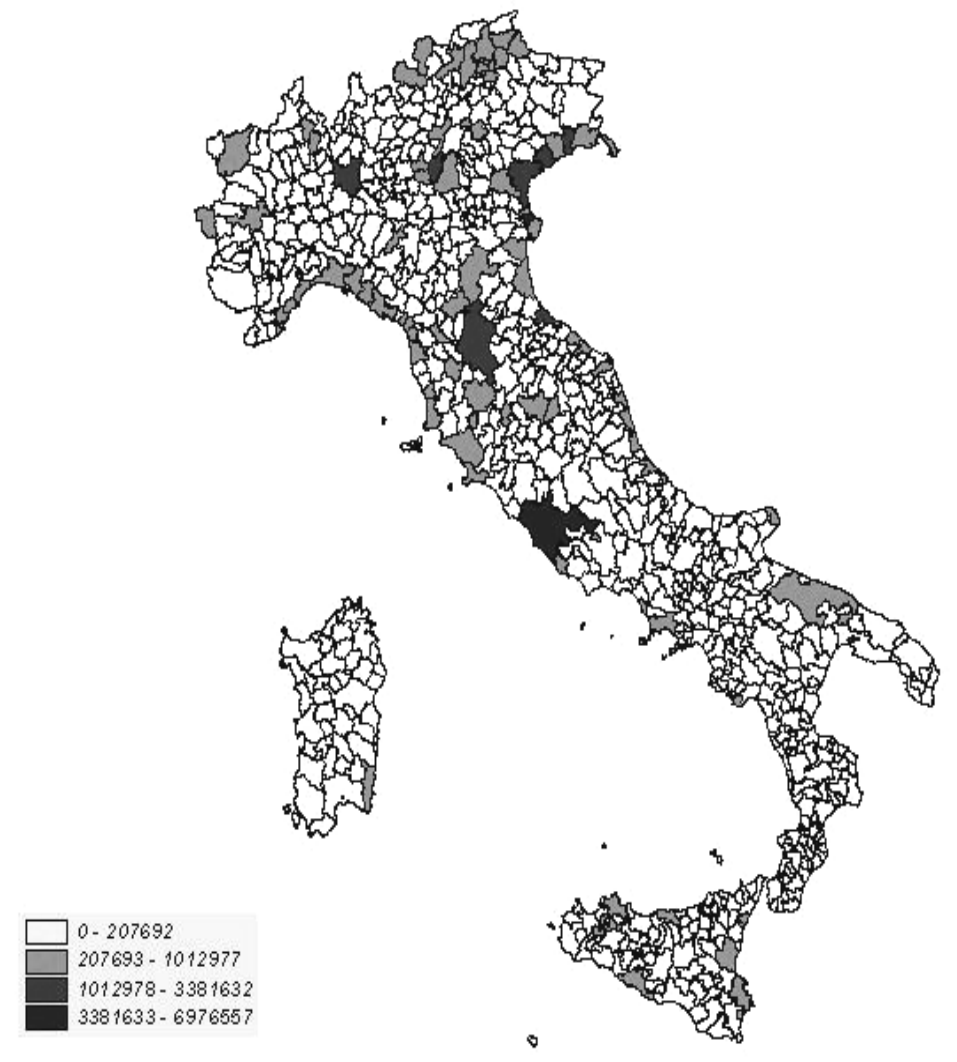

Fonte: nostra elaborazione su dati UIC, 2001;

\footnotetext{
${ }^{66}$ Dato che le elaborazioni sono state effettuate a livello di sistema locale è stato necessario armonizzare i dati territoriali, dato che i dati relativi alla spesa turistica, arrivi e presenze, viaggiatori italiani all'esterno per provincia di residenza sono registrati a livello provinciale. Al tal fine si è proceduto con il seguente metodo, si è ripartito ogni dato provinciale per comune e successivamente si è aggregato ogni comune per sistema locale. Per qualsiasi approfondimento si rimanda alla prima parte di questo capitolo relativo alle fonti dei dati.

${ }^{67}$ La prima classe registra arrivi fino a 200.000 , la seconda fino ad un 1.000.000, la terza fino a 3.300 .000 circa e l'ultima registra livelli fino a quasi 7.000.000. Gli arrivi sono registrati annualmente.

${ }^{68} \mathrm{Al}$ fine di analizzare unità territoriali che presentano patterns territoriali diversi sarebbe più corretto dividere le analisi per zone diverse. Per esempio in questo caso calcolare un indice di concentrazione su base porta probabilmente a sottostimare il fenomeno nel sud Italia. Lo stesso problema viene analizzato in Sforzi (1997a).
} 
La mappa degli arrivi totali non presenta una distribuzione territoriale simile a quella dell'offerta turistica del paragrafo precedente. Gli arrivi dei SLL ponderati sui lavoratori della filiera, dimostrano, invece, patterns simili a quelli della mappa 2.4 degli indici di concentrazione. Questo, d'altronde, risulta un indice di concentrazione degli arrivi per sistema locale del lavoro. La figura 2.13 evidenzia come emergono anche i sistemi locali del trentino e quelli del centro Italia, inoltre il sud è maggiormente presente. La distribuzione dimostra come le performance siano maggiormente legate al livello della struttura dell'offerta. Questo indice, essendo relativo, sottostima i grandi comuni, per esempio, Roma che risulta uscire dai sistemi che registrano il maggior numero di arrivi.

Figura 2.13: Arrivi totali ponderati sulla filiera;

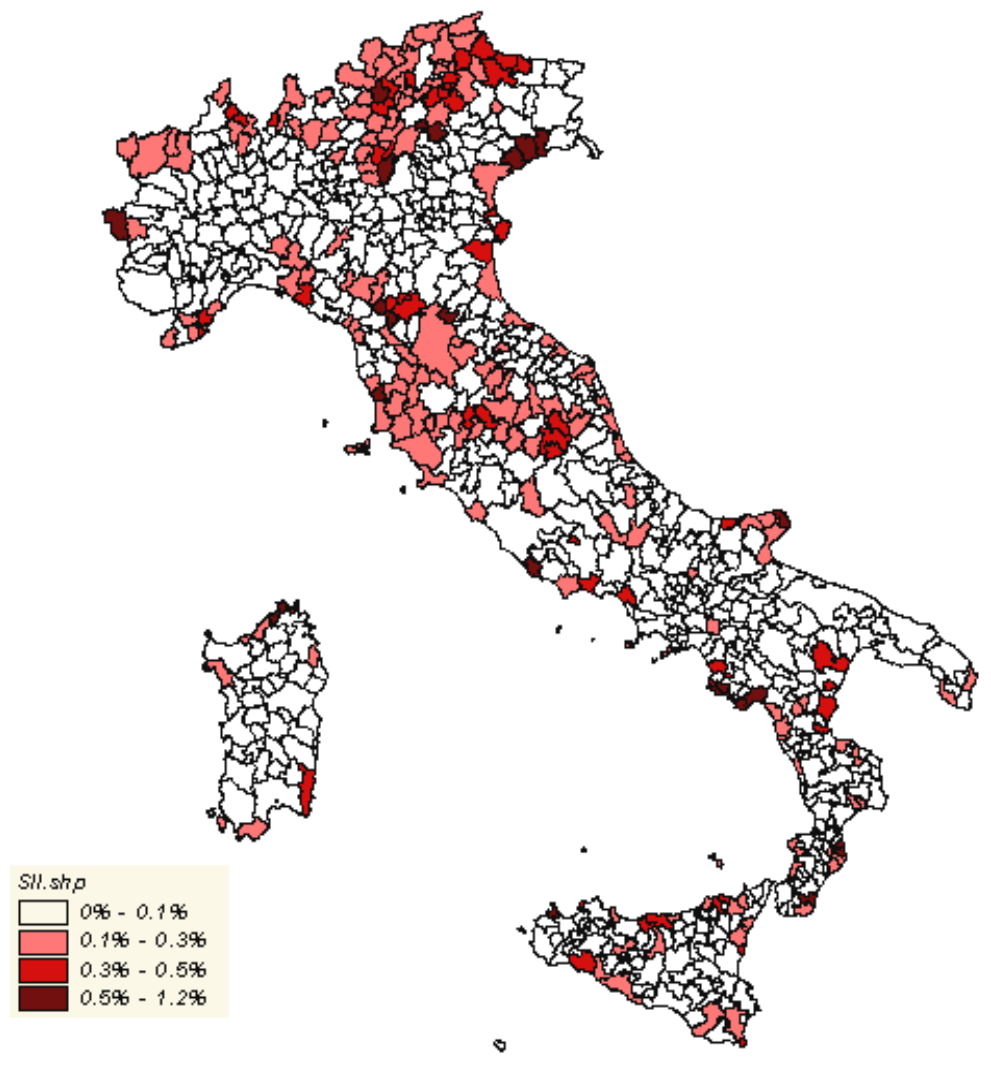

Fonte: nostra elaborazione su dati UIC, 2001;

Agli stessi risultati si perviene analizzando la spesa totale degli italiani e degli stranieri e poi relativizzando le analisi in base ai lavoratori della filiera. A tal fine si presenta l'ultima mappa relativa all'analisi della domanda. La figura 2.14 presenta la spesa relativa dei turisti ponderati per i lavoratori della filiera come proposta nel paragrafo precedente. I patterns territoriali sono ancora simili a quelli dell'offerta e strettamente legati alle performance degli stessi sistemi locali. Infine, questo tipo di analisi ci ha permesso di evidenziare come la struttura dell'offerta sia direttamente relazionata al rendimento per quanto riguardo gli arrivi e la spesa relativi dei sistemi locali stessi. 
Figura 2.14: Spesa relativa dei turisti in Italia in base ai lavoratori nella filiera;

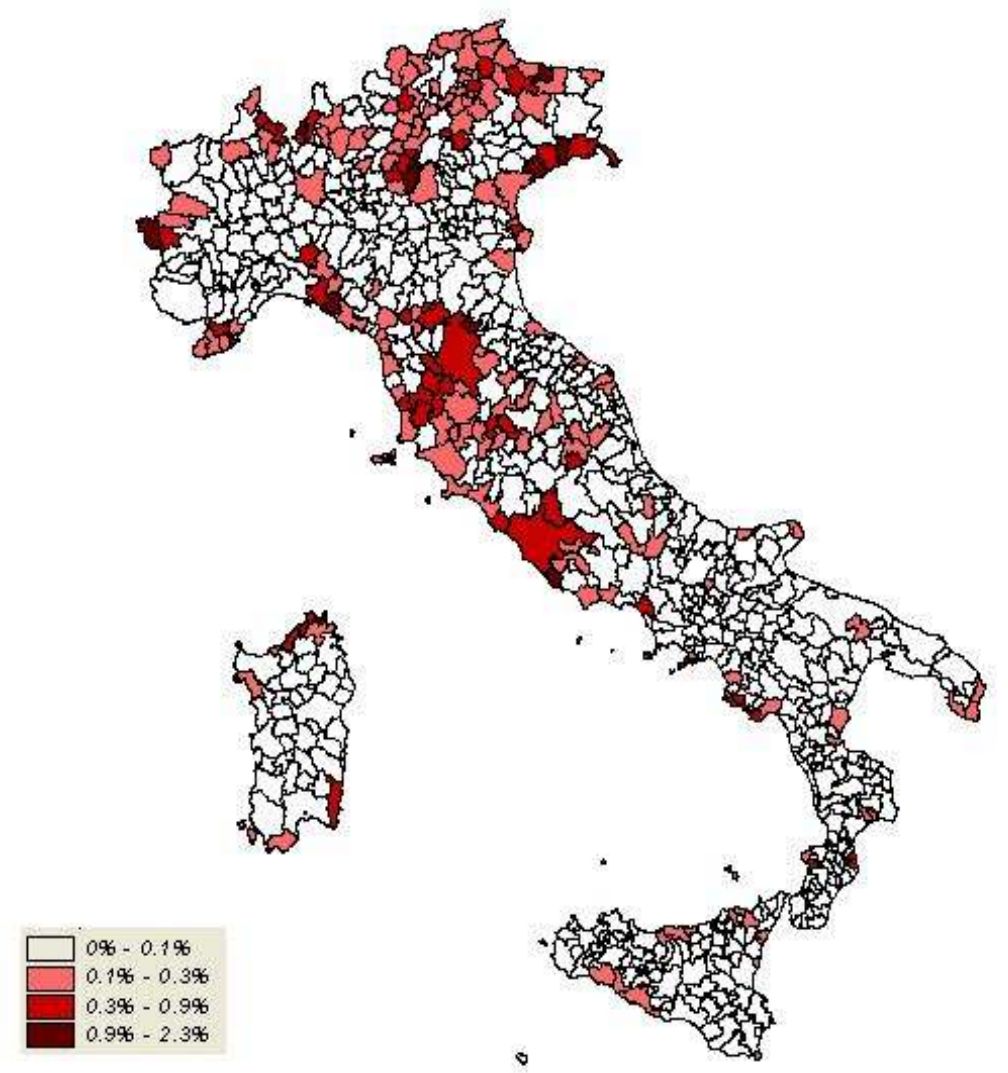

Fonte: nostra elaborazione su dati UIC e Istat, 2001;

Concludendo, questo paragrafo ci ha permesso di proporre un panorama delle destinazioni turistiche italiane per sistema locale. Questo non solo risulta di facile comprensione visiva, ma permette di approfondire le analisi con proxi scelta ad hoc da parte del ricercatore per evidenziare aspetti diversi del fenomeno in oggetto. Si ricorda, infine, che l'obiettivo di questo paragrafo non era quello di proporre un panorama esaustivo della domanda turistica italiana, ma principalmente quello di confrontare i patterns territoriali della domanda con quella dell'offerta. 


\subsubsection{Una Cluster Analysis sui SLT}

Al fine di evidenziare diverse tassonomie dei sistemi locali turistici evidenziati, in questo paragrafo applicheremo una cluster analysis ai circa 300 SLT evidenziati in base l'indice di concentrazione calcolato sulla filiera allargata. Tale tassonomia sarà utile non solo per evidenziare diverse caratteristiche dei sistemi locali analizzati, ma anche per proporre classi di performance diverse utili per le analisi dei capitoli successivi.

In particolare, applichiamo una K-Means Cluster Analysis (Dillon e Goldstein 1984) tramite il software SPSS. Questa procedura ci permette di identificare gruppi di osservazioni basate su caratteristiche selezionate a priori. Inoltre, la metodologia utilizza un algoritmo che permette di maneggiare un grande numero di casi. La k-means è una delle metodologie di analisi cluster che permette di suddividere un gruppo di osservazioni (sistemi locali del lavoro) in $\mathrm{k}$ gruppi. Questo permette di formare gruppi omogenei a partire dalle caratteristiche individuali delle osservazioni e minimizza la distanza dal più vicino centroide. Questo assegna ad ogni osservazione un gruppo minimizzando la distanza dal centro del gruppo di appartenenza (Dillon e Goldstein 1984:186-187).

Utilizziamo a tal fine una matrice della struttura della filiera allargata proposta nelle pagine precedenti. Ogni riga rappresenta ognuno dei 269 SLT individuati precedentemente che registrano un indice di concentrazione (sulla filiera) maggiore di 1.1, al fine di rendere l'analisi più robusta. Ogni colonna individua la percentuale di addetti nel sistema locale in quella attività della filiera allargata sul totale degli addetti della filiera stessa. Inoltre, aggiungiamo nella prima colonna il valore dell'indice di concentrazione nella filiera per collegare l'indice di specializzazione (su addetti) con la struttura dell'offerta.

La K-means produce 5 gruppi omogenei di sistemi locali turistici ${ }^{69}$. Il cluster \#2 è composto da circa 157 sistemi locali del lavoro e risulta essere il più numeroso. È il cluster che risulta maggiormente diversificato, inoltre, è il più specializzato nei treni, trasporti e nelle altre infrastrutture. D'altro canto ha il valore dell'indice di concentrazione più basso, ma come ricordato presenta valori del $L Q$ su quasi tutta la filiera maggiori dell'unità. È composto da grandi città d'arte come Roma, Firenze e Venezia, ma anche centri di più modeste dimensioni come Pisa, Siena, Orvieto, Spoleto e Assisi.

Il cluster \#4 è il secondo più numeroso e registra livelli dell'indice di concentrazione più alti che il gruppo precedente. D'altro canto, non ha un livello di infrastrutture simile e risulta specializzato solo in alcune parti della filiera come alloggiamento, e attività ricreative. In questo gruppo si trovano medi-piccoli centri dove il turismo è una delle attività principali come Rimini, Orbetello, Cattolica, Riva del Garda e piccoli centri rurali come Gaiole in Chianti, Manciano, San Quirico D'orcia.

Gli altri tre gruppi registrano insieme 100 sistemi locali (il cluster \#5 ha solo 4 SLL). Questo gruppo è quello maggiormente specializzato, con indici di concentrazione tra i più alti. Questi SLT sono quelli maggiormente specializzati in parti della filiera turistica in maniera esclusiva (con il più alto indice di concentrazione). A nostro avviso questi SLT possono essere considerati come i più probabili distretti turistici, così come presentati nella prima parte di questo scritto. Tuttavia, è necessario investigare maggiormente l'ambiente sociale e le relazioni della comunità locale. Questi SLT possono essere suddivisi in due

${ }^{69} \mathrm{Il}$ numero dei cluster è determinato a priori. Un approccio ottimale non esiste ed il numero di gruppi può influenzare i risultati. Per questa ragione abbiamo prima effettuato una analisi della sensibilità dei gruppi, cambiando il loro numero e i risultati si sono dimostrati robusti. Abbiamo, poi, effettuato anche una Hierarchical Cluster, che è una procedura che sviluppa il numero di gruppi ottimali. Il dendogramma che fornisce la Hierarchical Cluster ci ha mostrato che 5 cluster sono il numerodo di gruppi ottimali per l'analisi. 
sottogruppi. Il primo è caratterizzato nella prima parte della filiera: quindi trasporti e alloggiamento (60-55). Il secondo è basato invece sulla seconda parte della filiera: quindi ristorazione e attività ricreative (55-93).

Nella figura 2.6 si presentano sinteticamente i risultati della k-means cluster analysis con le differenze tra i gruppi: I risultati più eterogenei sono stati sottolineati per facilitarne l'evidenza e l'interpretazione.

Tabella 2.6: K-Means Cluster Analysis

\begin{tabular}{|c|c|c|c|c|c|}
\hline \multirow[b]{2}{*}{ Osservazioni 269} & \multicolumn{5}{|c|}{ Clusters } \\
\hline & $1(35)$ & $2(157)$ & $3(16)$ & $4(56)$ & $5(4)$ \\
\hline LQ sulla filiera allargata & $\underline{3,03}$ & $\underline{1,37}$ & $\underline{4,24}$ & $\underline{2,05}$ & $\underline{5,48}$ \\
\hline 60.1- Trasporti ferroviari &, 01 &,$\underline{04}$ &, 00 &,$\underline{02}$ &, 00 \\
\hline 60.2- Altri trasporti terrestri &,$\underline{12}$ & 27 & , $\underline{09}$ &, 15 & ,04 \\
\hline 55.1- Alberghi & $\underline{35}$ & 11 & .40 & .24 & .59 \\
\hline 55.2- Campeggi ed altri alloggi per brevi soggiorni &,$\underline{10}$ &,$\underline{04}$ & ,13 & , 07 &,$\underline{08}$ \\
\hline 55.3- Ristoranti &,$\underline{18}$ & $\underline{21}$ & , 15 & ,21 & , 13 \\
\hline 55.4- Bar &,$\underline{15}$ &,$\underline{19}$ & , $\underline{09}$ &,$\underline{17}$ & , $\underline{09}$ \\
\hline $\begin{array}{l}63.3 \text { Attività delle agenzie di viaggio e degli operatori } \\
\text { turistici; }\end{array}$ &, 02 &, 02 & ,01 &,$\underline{02}$ & ,04 \\
\hline 70.2 Locazione beni immobili & ,02 &,$\underline{03}$ & 01 &,$\underline{03}$ & 01 \\
\hline $\begin{array}{l}\text { 92.3 Altre attività dello spettacolo, di intrattenimento e } \\
\text { divertimento }\end{array}$ & ,01 &,$\underline{03}$ & 01 &,$\underline{02}$ & 00 \\
\hline $\begin{array}{l}92.5 \text { Attività di biblioteche, archivi, musei ed altre } \\
\text { attività culturali }\end{array}$ &, 00 & ,01 & 00 &, 00 & 00 \\
\hline 92.7 Attività ricreative &,$\underline{02}$ & 01 & 01 & 01 & 00 \\
\hline 92.6 Attività sportive & 01 &,$\underline{02}$ & , $\underline{06}$ &,$\underline{02}$ & 01 \\
\hline $\begin{array}{l}\text { 26.1 Fabbricazione di vetro e di prodotti in vetro } \\
\text { (artigiani) }\end{array}$ &, 00 &, 00 & 00 &, 00 & 00 \\
\hline 62.1 Trasporti aerei di linea & 00 & 01 &, 01 &, 00 & 00 \\
\hline $\begin{array}{l}\text { 61.2 Trasporti per vie d'acqua interne (compresi i } \\
\text { trasporti lagunari) }\end{array}$ &, 00 & ,00 &, 00 &, 00 & 00 \\
\hline 71.1 Noleggio di autovetture &, 00 &, 00 & 00 &, 00 & 00 \\
\hline 61.1 Trasporti marittimi e costieri &, 00 & 00 & 00 &, 00 & 00 \\
\hline $\begin{array}{l}1.13 \text { Coltivazione di frutta, frutta a guscio, prodotti } \\
\text { destinati alla preparazione di bevande, spezie }\end{array}$ &, 00 &,$\underline{02}$ & ,00 &,$\underline{02}$ & ,00 \\
\hline
\end{tabular}

Fonte: nostra elaborazione;

Infine si presentano i risultati della cluster analisi presentati in tabella anche in forma grafica per permetterne una migliore interpretazione. Nella figura 2.15 si presentano i clusters individuati nel territorio italiano, suddivisi nei 5 gruppi. La distribuzione territoriale sembra non presentare dei patterns territoriali specifici. Risulta in ogni modo necessario a nostro avviso approfondire questo aspetto con modelli di analisi spaziali che svilupperemo nel prossimo capitolo. La divisione in clusters eterogenei ci permetterà di evidenziare se esistano performance diverse tra $\mathrm{i}$ clusters indicati ${ }^{70}$.

\footnotetext{
${ }^{70}$ La mappa sottolinea come il cluster numero 2 di colore giallo risulti il più numeroso, come evidenziato dall'analisi quantitativa. Rileviamo che le indicazioni su ogni cluster, sono indicazioni di massima e questo implica che non tutte le osservazioni che appartengono ad un gruppo hanno caratteristiche coincidenti con le caratteristiche del gruppo. A nostro avviso, per esempio la presenza di SLL di colore giallo, quindi diversificati, in prevalenza in Sardegna solleva alcuni dubbi.
} 
Figura 2.15: Sistemi Locali del lavoro in base alla K-means Cluster;

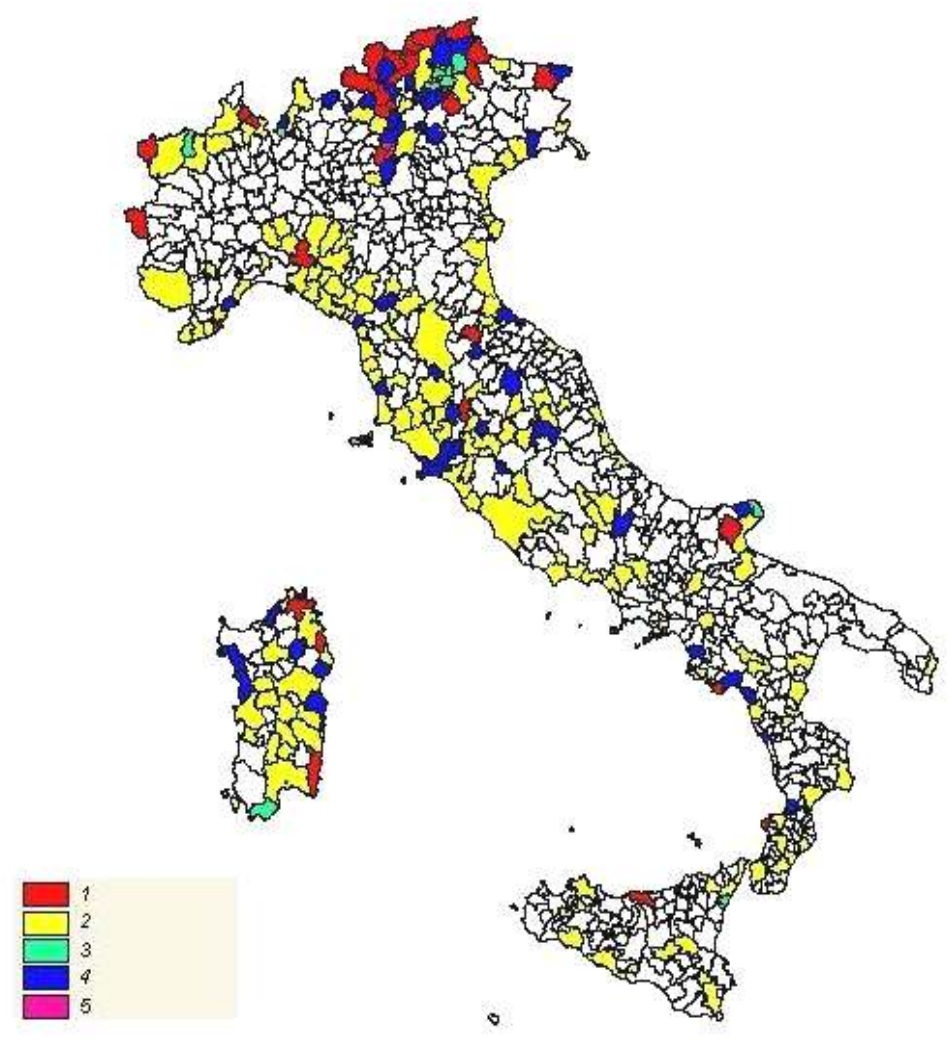

Fonte: nostra elaborazione su dati Istat, 2001;

\subsubsection{Una classificazione per analizzare i SLT}

Utilizzando l'approccio di Suzigan et al. (2004) presentiamo una classificazione dei sistemi locali al fine di proporre alcune indicazioni di massima sulle caratteristiche dei SLT. Gli autori propongono una classificazione dei sistemi locali in base al grado di specializzazione ed al contributo all'economia nazionale. Il coefficiente di localizzazione, nonostante sia ponderato ad un grado soglia, non cattura il livello di contributo all'industria nazionale. In altre parole, luoghi anche molto piccoli e mono-specializzati pesano in maniera superiore a luoghi che contribuiscono in maniera sostanziale all'industria turistica italiana. La classificazione a due dimensioni permette di risolvere il problema.

Mentre esiste un ampio riconoscimento sull'importanza dei sistemi locali di imprese, manca un accordo su come questi sistemi dovrebbero essere promossi e gestiti e su quali politiche dovrebbero essere implementate. La classificazione permette di focalizzarsi e differenziare l'approccio per quattro tipi di SLT ${ }^{71}$.

\footnotetext{
${ }^{71}$ In base alla specializzazione ed al contributo al comparto turistico italiano si classificano i SLT in quattro categorie: 1) Alto indice di concentrazione e basso contributo all'economia nazionale; 2) Medio-alto indice di concentrazione, ma alto contributo al turismo nazionale; 3) Basso contributo e basso indice di concentrazione; 4) Basso indice di concentrazione, ma alto contributo al turismo nazionale. Gli autori presentano, invece quattro categorie denominate: Vectors of local develompent, centers of industrial and regional development, embryonic local production systems, advanced vectors.
} 
Presentiamo nella tabella 6.2 alcuni dei SLT classificati in base a queste quattro tipologie.

La prima categoria ha un coefficiente di localizzazione molto alto, ma il contributo all'industria turistica italiana è limitato. Questi SLT sono quelle destinazioni monofunzionali piccole che basano la propria economia esclusivamente o quasi esclusivamente sulle attività turistiche.

La seconda categoria è composta invece da quei sistemi locali turistici che non solo registrano un alto coefficiente di localizzazione, ma contribuiscono in maniera importante all'industria nazionale. L'importanza di queste destinazioni le costituisce come centri turistici regionali o nazionali altamente specializzati.

I SLT che ricadono nella terza categoria hanno un basso coefficiente di concentrazione e un basso contributo al turismo nazionale. Sono dei SLT in cui il turismo è in fase di sviluppo.

L'ultima categoria è composta da SLT diversificati, che hanno coefficienti di localizzazione bassi, ma che danno il maggiore contributo al turismo nazionale. Si compone questo gruppo di destinazioni, affermate di medie-grande dimensioni, prevalentemente città d'arte come Roma, Venezia e Firenze.

Tabella 2.7: Tipologia ed esempi di SLT in Italia;

\begin{tabular}{|c|c|c|c|}
\hline & \multicolumn{2}{|c|}{ Contributo Italia } \\
\hline & & Low $(0,5 \%>x>0,1 \%)$ & High $(x>0,1 \%)$ \\
\hline \multirow[t]{2}{*}{ LQ } & $\begin{array}{l}\text { High } \\
(>1,5)\end{array}$ & $\begin{array}{c}\text { SLT di sviluppo locale } \\
\text { Limone Sul Garda, Positano } \\
\text { Malcesine, Amalfi } \\
\text { Canazei, Porto Azzurro } \\
\text { Nova Ponente, Vieste, Capri }\end{array}$ & $\begin{array}{c}\text { Centri turistici } \\
\text { Badia, Taormina, Saint-Vincent } \\
\text { Ischia, Ortisei, Sorrento, Alassio } \\
\text { Bormio, Montepulciano }\end{array}$ \\
\hline & $\begin{array}{l}\text { Low } \\
(1>x>1,5)\end{array}$ & $\begin{array}{c}\text { SLT a livello embrionale } \\
\text { Nuoro, Domodossola } \\
\text { Lamezia Terme } \\
\text { Follonica, Porretta Terme, Spoleto, } \\
\text { Pont-Saint-Martin, Orvieto, } \\
\text { Rosignano Marittimo }\end{array}$ & $\begin{array}{l}\quad \text { Destinazioni turistiche } \\
\text { Roma, Napoli, Firenze, Bologna, } \\
\text { Venezia, Genova, Padova, Verona, } \\
\text { Palermo, Cagliari, Bolzano, Trieste }\end{array}$ \\
\hline
\end{tabular}

Fonte: nostra elaborazione;

Le destinazioni turistiche affermate sono quei sistemi locali che hanno i maggiori problemi di sostenibilità turistica, congestione urbana e sovra-affluenza di turisti. In questi casi una maggiore destagionalità dell'offerta e un maggior sviluppo con i centri vicini potrebbe aiutare ad aumentare la permanenza dei visitatori e sviluppare collaborazioni a livello territoriale tramite itinerari turistici.

I centri turistici sono quei SLT, ormai riconosciuti, che si basano fortemente sull'industria turistica. Questi ormai percorrono delle vie di sviluppo ottimali e forniscono un contributo importante all'industria nazionale. Quindi sono i SLT che meritano la 
maggiore attenzione dai policy-makers. Lo sviluppo di tali centri dovrebbe mirare maggiormente alla sviluppo di ulteriori anelli della filiera, come le attività di marketing e la certificazione di qualità. Inoltre lo sviluppo di corsi di formazione e educazione potrebbe aumentare le capacità tecniche e le funzionalità delle destinazioni.

I SLT a livello embrionale sono i più difficili da promuovere. Questi SLT ancora non hanno preso una strada definita e nonostante il turismo sia un settore importante dell'industria locale, il contributo alla economia della nazione è ancora limitato. L'errore più comune potrebbe essere quello di sviluppare le stesse strategie dei centri turistici, che però possono avere caratteristiche proprie ed uniche e non ripetibili tramite politiche, almeno nel breve termine. A nostro avviso questo gruppo, che è il più numeroso, è quello sui cui sviluppare per ogni caso una politica ad hoc, sia per quanto riguarda la presenza della filiera a livello locale sia per quanto riguarda le sinergie con le destinazioni turistiche limitrofe.

L'ultimo gruppo i vettori di sviluppo locale sono i SLT che registrano un coefficiente di localizzazione più alto in assoluto, sono piccoli e medi centri che contribuiscono relativamente all'industria nazionale. Lo sviluppo di tali centri dovrebbe mirare allo sviluppo di tutti gli anelli della filiera a livello di rete e principalmente creare collaborazioni con destinazioni turistiche vicine, al fine di aumentare il peso locale dell'industria.

\subsection{CONCLUSIONI}

Lo scopo di questo capitolo è stato quello di identificare i Sistemi Locali Turistici italiani. Abbiamo individuato i SLT in Italia e verificato la coerenza dei risultati con il panorama italiano delle destinazioni turistiche.

Innanzitutto la scelta di utilizzare la teoria distrettuale ed i sistemi locali del lavoro si è dimostrata soddisfacente. Sia il primo studio che la seconda analisi hanno evidenziato come i SLT registrino una percentuale di piccole e medie imprese sostanziale e confermino l'adeguatezza del modello distrettuale per lo studio dell'industria turistica poiché basata su un tessuto di piccole e medie imprese localizzate.

La rassegna sullo stato dell'arte ha sottolineato come non fosse ancora sviluppata un'individuazione quantitativa dei SLT di questo tipo. Da un punto di vista l'ISTAT fornisce alcune elaborazioni sui distretti turistici, $\mathrm{i}$ quali però vengono identificati in base ad una specializzazione esclusiva sul turismo. Altri studi invece (IRPET) rilevano anche le relazioni dei sistemi turistici con gli altri settori, ma non sono estesi a livello nazionale (solo Regione Toscana).

Unico lavoro che supera questi limiti è il contributo di Sforzi (1997b), i cui risultati sono simili alle nostre analisi, che si propongono come una sua continuazione temporale. Infatti, questo contributo prende in considerazione l'evoluzione dei SLT dal 1981 al 1991 mentre la nostra analisi è sviluppata nel decennio successivo con una analisi longitudinale in base a tre censimenti nazionali: 1991, 1996, 2001.

Tuttavia, lo studio di Sforzi, analizza i servizi per i consumatori (Alberghi, ristoranti, caffè, agenzie di viaggio, etc.) e non si focalizza su un maggior numero di attività dedicate ai turisti. Abbiamo, per questo, proposto e costruito una filiera turistica ad hoc al fine di individuare i SLT in base ad attività scelte su misura, prendendo spunto da contributi istituzionali della Commissione Europea e della OECD. La filiera ha dato risultati 
soddisfacenti e si configura come un contributo originale. Si è evidenziato, poi, l'esistenza di interrelazioni tra l'industria turistica dei SLT con altri settori (agricoltura, industria, commercio), in particolare le attività manifatturiere.

Abbiamo analizzato i SLT, in maniera sintetica da parte della domanda ed in modo più esaustivo da parte della struttura dell'offerta, si è quindi proposto una classificazione tramite la cluster analysis ed in base al contributo alla economia nazionale.

Questo capitolo risulta una solida base quantitativa di individuazione dei sistemi locali turistici in Italia aggiornata al censimento del 2001. Inoltre, propone un approfondimento specifico sulla loro evoluzione nell'ultimo decennio (1991-2001).

I SLT individuati rappresentano le destinazioni turistiche italiane e tramite le applicazioni GIS siamo stati in grado di presentare molteplici mappe sulla loro evoluzione temporale e sulla variabilità dei risultati in base alla definizione di turismo che si adotta. Ulteriori studi sono sicuramente necessari, ma l'utilizzo dei SLL e l'applicabilità della teoria distrettuale hanno portato ad una identificazione rigorosa dei sistemi locali turistici in Italia che si configura come una base di partenza per analisi di tipo più specifico per gli operatori e gli studiosi del turismo.

Nella seconda parte di questa tesi applicheremo dei modelli di econometria spaziale al fine di evidenziare se questi sistemi di imprese beneficiano di vantaggi competitivi (economie esterne, risorse naturali, spillovers). In particolare ci interrogheremo su come competono questi sistemi locali e a cosa è dovuta la loro crescita. Questa ed altre domande troveranno in parte delle risposte nei prossimi capitoli. 
PARTE SECONDA 


\section{CAPitolo III}

\section{LE FONTI DELLA COMPETITIVITÀ DEI SISTEMI PRODUTTIVI LOCALI: RISORSE NATURALI, ECONOMIE ESTERNE E SPILLOVERS}

\subsection{PREMESSA}

Al fine di analizzare le fonti di competitività dei sistemi locali si presuppone che esistano due tipi di fonti competitive. Queste due forze spiegano perché ed in che modo le imprese hanno vantaggi ad agglomerarsi nello spazio e situarsi in aree limitrofe: le risorse naturali e le economie esterne.

Il vantaggio competitivo dovuto alle di risorse naturali risulta poco trattato negli studi regionali, geografici e nelle analisi sulle agglomerazioni di imprese in generis. Ci riferiamo, in particolare, alla dotazione di risorse naturali, di cui una specifica area dispone a differenza di altre. Risaliamo in questa parte ai contributi di Marshall sulla dotazione delle risorse naturali delle agglomerazioni di imprese per poi esporre una rassegna degli studi che cercano di affrontare il fenomeno dell'agglomerazione come motivato congiuntamente dalla dotazione di risorse naturali e dai vantaggi di agglomerazione di tipo economico (Glaeser et al., 1992; Kim, 1995, 1999; Porter, 2003). Ne approfondiamo gli aspetti relativi nel prossimo paragrafo.

Presentiamo poi le economie esterne di localizzazione, urbanizzazione e rete come elementi di competitività che influenzano profondamente i sistemi produttivi locali. Il principale obiettivo è di proporre una classificazione delle economie esterne, al fine di poter misurare questi vantaggi nella seconda parte di questa tesi. Vedremo che non esiste una classificazione unica e, soprattutto, diverse discipline apportano aspetti e punti di vista differenti. Si richiamano gli scritti di Alfred Marshall sulle note economie interne ed esterne per poi approfondire i contributi dell'Economia Urbana e Regionale per l'analisi dello sviluppo della città e delle regioni tramite gli spillovers e le economie di rete. Questo perché la città risulterà una delle unità di analisi nella seconda parte di questo lavoro come destinazione turistica e città d'arte ${ }^{72}$. Emerge quindi la necessità, di applicare dei modelli di analisi che siano degli ibridi tra lo studio delle economie esterne distrettuali (localizzazione), la competitività delle città (economie di urbanizzazione), delle reti di città (economie a rete) e delle regioni (spillovers).

Sviluppiamo nel terzo paragrafo alcuni cenni al contributo di Marshall sulle economie interne ed esterne alle imprese per poi definire le economie di localizzazione.

\footnotetext{
${ }^{72}$ Le città d'arte, per esempio, sono difficilmente analizzabili tramite il modello del distretto dato che quelli marshalliani erano soprattutto singole parti o quartieri di una città.
} 
Nel quarto paragrafo introduciamo i contributi della Economia Urbana e Regionale (Weber, 1928; Ohlin, 1933; Hoover, 1937). Definiamo, qui, le economie di agglomerazione à la Hoover, le economie di rete relative alla teoria delle reti di città e spillovers spaziali tipici degli ultimi sviluppi della Economia Regionale ${ }^{73}$. Infine si presenta uno schema riassuntivo.

\subsection{LA DOTAZIONE DI RISORSE NATURALI}

Marshall in Principi di Economia elenca i fattori di produzione come principalmente in lavoro, capitale e terra: "the agents of production are commonly classed as Land, Labour and Capital. By Land is meant the material and the forces which Nature gives freely for man's aid, in land and water, in air and light and heat'. (Marshall, 1980, Book IV, Capitolo 1:i)

L'autore prosegue affermando che in verità esistono solo due fattori di produzione l'uomo e le risorse naturali, le altre sono solo un effetto dell'insieme del lavoro dell'uomo e sulle risorse naturali e pone poi l'attenzione sulla centralità dell'uomo sia per la produzione sia per il consumo.

"In a sense there are only two agents of production, nature and man. Capital and organization are the result of the work of man aided by nature, and directed by bis power of forecasting the future and his willingness to make provision for it. If the character and powers of nature and of man be given, the growth of wealth and knowledge and organization follow from them as effect from cause. But on the other hand man is himself largely formed by his surroundings, in which nature plays a great part: and thus from every point of view man is the centre of the problem of production as well as that of consumption". (Marshall, 1980, Book IV, Capitolo 1:ii).

Le economie esterne sono uno dei principali vantaggi dei sistemi di imprese e sono riconosciute come importanti fattori che influenzano le agglomerazione delle attività economiche. Tuttavia lo stesso Marshall identifica le risorse naturali come una delle cause principali di questi vantaggi:

"Many various causes have led to the localization of industries; but the chief causes have been physical conditions; such as the character of the climate and the soil, the existence of mines and quarries in the neighbourbood, or within easy access by land or water. Thus metallic industries have generally been either near mines or in places where fuel was cheap. The iron industries in England first sought those districts in which charcoal was plentiful, and afterwards they went to neighbourbood of collieries. Staffordshire makes many kinds of pottery, all the materials of which are imported from a long distance; but she has cheap coal and excellent clay for making the heary 'saggars' or boxes in which the pottery is placed while being fired. Straw plaiting has its chief home in Bedfordshire, where straw bas just the right proportion of silex to give strength without brittleness; and Buckinghamshire beeches have afforded the material for the Wycombe chair making. The Sheffield cutlery trade is due chiefly to the excellent grit of which its grindstones are made." (Marshall, 1920:268-269).

In questo noto passaggio, l'autore sottolinea come la localizzazione dell'industria nel distretto della coltelleria ${ }^{74}$ di Sheffield sia dovuta alla qualità delle pietre della zona.

Un altro lavoro che dibatte il medesimo tema è quello di Kim (1999). L'autore analizza il ruolo delle risorse naturali nelle agglomerazioni geografiche degli Stati Uniti e

\footnotetext{
${ }^{73}$ In un recente contributo della Harvard University, Baptista (1998) classifica gli studi sulle economie esterne successivi a Marshall in cinque filoni principali: il primo si riferisce alla Urban and Regional Economics (Weber 1928; Ohlin 1933; Hover 1937), il secondo alla Geography (Amin e Goddard 1986; Conti et al. 1995, etc). Il terzo richiama gli studi di Historian and Economic Historian alla Jacobs (1969). Il quarto risale ad uno sviluppo successivo del precedente che l'autore titola Path dependent to economics di Arthur (1990), Krugman (1991, 1993) e Porter $(1990,1998)$ ed infine il quinto si dedica alla New theory growth di Romer $(1986,1990)$.

${ }^{74}$ Cutlery district.
} 
sottolinea come queste ${ }^{75}$ siano causa di numerose mutazioni geografiche nelle industrie manifatturiere dal 1880 e il 1987. La dotazione di risorse naturali si configura come un fattore limite all'importanza delle esternalità nella geografia economica degli Stati Uniti.

In un precedente articolo (1995) lo stesso autore, analizzando la specializzazione regionale degli Stati Uniti, registra che anche se dal 1880 al 2000 esiste un incremento della concentrazione delle industrie, il trend risulta invertito dal 1950 in poi. Le industrie sono concentrate in misura mediamente minore e l'indice di concentrazione è maggiore per le manifatture tradizionali.

L'autore afferma infine come gli studi riguardanti la concentrazione geografica e la specializzazione regionale non riescono a risolvere il problema, di difficile soluzione, di decomporre l'effetto di concentrazione proveniente dall'esternalità di un industria da quello delle risorse naturali della stessa industria (ibidem, 1995:981 e ss.).

Una classificazione interessante è proposta da Porter. In un recente scritto l'autore analizza la competitività delle regioni americane e delle aree sub-regionali, approfondendo le fonti di competitività, basandosi però anche sui vantaggi provocati dalla dotazione di risorse naturali. L'autore evidenzia come le industrie negli Stati Uniti si possano classificare in tre tipologie: local industries, resource dependent industries e traded industries. La prima tipologia è caratterizzata da un'occupazione pressoché proporzionale alla popolazione e si sviluppa in base alla richiesta del mercato interno di riferimento. Troviamo in questa prima tipologia industriale: sanità, commercio, costruzioni, editoria, etc. Non esiste un effetto natural endowments in questo tipo di industrie.

La seconda tipologia è invece quella denominata resource dependent. L'occupazione nell'industria è principalmente concentrata dove sono necessarie alcune risorse naturali, queste industrie competono anche con attori nazionali ed internazionali. Alcuni esempi sono l'estrazione dell'uranio, la lavorazione dello zucchero ed i trasporti sui grandi laghi ${ }^{76}$. Queste industrie sono estremamente dipendenti dalle risorse naturali vicino alle quali sono localizzate.

L'ultimo tipo di industrie comprende quelle definite traded industries, che sono legate ad un certo tipo di risorse nel territorio, ma risultano concentrate per motivi maggiormente connessi alla competizione ed alla struttura dell'industria. L'occupazione è concentrata in maniera eterogenea a livello territoriale. Esistono da questo punto di vista dei vantaggi legati alla competizione ( $\mathrm{ma}$ anche alle risorse) e al suo sviluppo a livello nazionale ed internazionale. In questa tipologia sono elencate le principali industrie americane, ma soprattutto quella del vino (concentrata in California, nella Napa Valley) e l'industria turistica concentrata in maniera eterogenea. (Porter, 2003:575)

Porter prosegue sottolineando come una delle più sorprendenti caratteristiche di queste economie regionali sia la presenza di cluster o di concentrazioni geografiche di industrie collegate. Esempi di questo tipo sono i servizi finanziari in New York, la produzione di apparecchiature mediche in Boston e le ICT in Austin, Texas ed ovviamente la Silicon Valley.

Questa breve rassegna evidenzia come spesso nell'analisi economica (quantitativa) della localizzazione di imprese, il ruolo delle risorse naturali è stato spesso sottovalutato e

\footnotetext{
${ }^{75}$ Le risorse naturali vengono considerate come quelle relative a capitale, forza lavoro e risorse naturali (industrie estrattive, etc.).

76 "Employment in these industries is located primarily where the needed natural resources are found, but these industries compete with other domestic and international locations. Examples of such industries include uranium ore, logging, beet sugar, and freight transportation on the Great Lakes." Porter (2003:559).
} 
posto in secondo piano. Pochi sono gli studi che analizzano questo aspetto e che riescono a misurare l'effetto di sinergia tra vantaggi naturali ed economie di localizzazione.

Dello stesso parere sono Ellisson e Glaeser (1997), che approfondiscono il legame tra gli spillovers ${ }^{77}$ spaziali e la dotazione di risorse naturali per il settore manifatturiero degli Stati Uniti. Riguardo alle risorse naturali, i due autori si riferiscono in particolare a quelle forze che, per esempio, spingono l'industria del vino a concentrarsi in una zona della California, la Napa Valley: il clima favorevole per la crescita dell'uva, la fertilità del terreno, etc. I risultati dell'analisi evidenziano infatti come l'industria vitivinicola californiana sia una delle industrie americane maggiormente concentrata geograficamente.

Da questo punto di vista, come sottolineano gli autori, mentre gli spillovers spaziali hanno una rilevante importanza negli studi internazionali sulle agglomerazioni, "le risorse naturali o natural endowments hanno ricevuto meno attenzione di quello che effettivamente meritino data la loro importanza empirica" (ibidem:921) ${ }^{78}$.

La dotazione di risorse naturali o di fattori di produzione non può essere considerata come la unica determinante per la competitività di un sistema locale, ma si configura come una condizione necessaria e non sufficiente in alcune attività come quella turistica.

Porter sottolinea tale aspetto ed inserisce le condizioni dei fattori e le risorse naturali nel diamante porteriano per l'analisi della competitività a scala nazionale (Porter, 1990) e locale (ibidem, 1998). L'autore rileva come la teoria neoclassica spieghi il successo di particolari industrie, rifacendosi ai cosiddetti fattori della produzione quali la terra, il lavoro ed appunto le risorse naturali, tramite l'acquisizione di un vantaggio comparato nelle industrie che massimizzano l'uso di tali ricchezze (vantaggi comparativi à la Ricardo). In particolare Porter rileva come la posizione di una nazione nel quadro dei fattori della produzione - quali la forza lavoro qualificata o le infrastrutture - sia necessaria per competere in un dato settore industriale e afferma "secondo la teoria economica convenzionale, $i$ fattori della produzione - lavoro, terra, risorse naturali, capitali, infrastrutture - determinano i movimenti commerciali internazionali. Una nazione esporta le merci la cui produrione impiega in misura massima $i$ fattori di cui essa è relativamente ben dotata. Questa dottrina le cui origini risalgono ad Adam Smith e David Ricardo, ed è incorporata nell'economia classica, nell'ipotesi migliore è incompleta, e nella maggiore è scorretta" (Porter, 1998; vers. Italiana, 2000:169).

Nelle industrie avanzate continua l'autore, una nazione non eredita i fattori della produzione più importanti, come le risorse umane specializzate o una base di conoscenze scientifiche: li crea. Da questo punto di vista gli svantaggi possono trasformarsi in vantaggi e viceversa. Quando vi è abbondante offerta di materie prime o di manodopera a basso costo, le imprese possono puntare esclusivamente su questi vantaggi e addirittura servirsene in maniera inefficiente ${ }^{79}$.

Pensiamo che emerga, quindi, la necessità di sviluppare maggiormente un approccio natural resources-based al fine di analizzare la fonte di competitività dei sistemi localizzati di imprese. In particolare la dotazione di risorse culturali e naturali può costituire un differenziale importante per i sistemi locali turistici. In sintesi, ci interroghiamo quanto la

\footnotetext{
77 L'idea principale è che "la prossimità geografica faciliti la trasmissione di idee e la diffusione di conoscenza. [...] attraverso l'imitazione, lo spionaggio, e lo scambio di manodopera tra le imprese, le idee sono velocemente sviluppate e diffuse tra le imprese vicine" (Glaeser et al., 1992:1127). Per spillovers spaziali gli autori intendono sia quelli fisici che intellettuali o di conoscenza (Glaeser et al., 1992).

78 Traduzione propria.

79 Da questo punto di vista, sia a livello locale, che internazionale, le altre "forze" della competizione influenzano lo sviluppo di capacità competitive in un'industria (struttura della domanda, industrie collegate e di supporto, rivalità, strategia e struttura del mercato).
} 
loro competitività è dovuta esclusivamente alla struttura dell'offerta o congiuntamente alle risorse naturali del luogo. La dotazione di patrimonio artistico culturale ed ambientale può evidenziare un effetto natural endowment utile per spiegare le fonti di competitività locali.

\subsection{CENNI SULLE ECONOMIE DI LOCALIZZAZIONE}

Marshall è stato uno dei primi economisti a scrivere sui distretti industriali. Osservando le agglomerazioni di piccole e medie imprese nelle città inglesi del $18^{\circ}$ secolo, sviluppò il concetto di economie esterne. In un famoso passaggio l'economista osserva (1920:271):

"When an industry has chosen a locality for itself it is likely to stay there long: so great are the advantage which people following the same skilled trade get from neighbourhood to one another. The mystery of the trade become no mysteries; but are as it were in the air, and children learns many of them unconsciously.. Employers are apt to resort to any place where they are likely to find a good choice of workers with the special skill which they require. The advantages of variety of employment are combined with those of localization industries in some of our manufacturing towns and this is a chief cause of their continued economic growth".

Marshall suddivide $i$ rendimenti crescenti nella produzione in due tipologie: economie interne ed esterne. Le economie interne sono prodotte e sviluppate all'interno di un'impresa. Quelle esterne descrivono una situazione in cui l'impresa riceve vantaggi al di fuori del "tetto dell'impresa". L'esistenza di queste economie esterne permette la costituzione di vantaggi competitivi all'industria localizzata.

I distrettualisti riprendono la classificazione di Marshall e utilizzano le economie esterne come uno degli strumenti concettuali necessari per interpretare le caratteristiche di efficienza collettiva di sistemi di imprese industriali. Citando ancora Marshall (1920:221): “...possiamo dividere le economie che provengono da un aumento della scala di produrione di ogni specie di beni, in due classi - in primo luogo, quelle che dipendono dallo sviluppo generale dell'industria; e, in secondo luogo, quelle che dipendono dalle risorse di singole case di affari impegnate in quella produrione, dalla loro organizzazione e dall'efficienza del loro management. Chiameremo le prime economie esterne, e le seconde economie interne"; per poi analizzare quelle economie esterne tipiche dei distretti industriali "che si possono spesso ottenere mediante la concentrazione di parecchie piccole imprese di natura simile in località particolari; o, come si dice comunemente, mediante la localizzazione dell'industria" (ibidem).

Bellandi (1987) riferendosi a Marshall propone una classificazione delle economie di localizzazione in quattro tipi: a) una maggiore specializzazione basata sulla divisione del lavoro; b) maggiore circolazione di informazioni, c) maggiore formazione e d) maggiore capacità di innovazione. In un attuale contributo (2003), l'autore classifica, invece, le economie esterne sulla base della fonte principale in: specializzazione, apprendimento e creatività. Nel primo caso si tratta di vantaggi di uso di migliori capacità produttive, nel secondo di sviluppo di capacità umane, nel terzo caso di nuove capacità tecniche (Bellandi 2003:103).

Le economie di localizzazione à la Marshall sono comunemente riconosciute in letteratura come provenienti da tre fonti distinte: mano d'opera qualificata, fornitori specializzati e spillovers di conoscenza (Rosenthal e Strange 2003). 
Capitolo terzo

\subsection{IL CONTRIBUTO DELL'ECONOMIA URBANA E REGIONALE}

\subsubsection{Le economie di agglomerazione}

Risalendo al contributo di Weber (1933), l'Economia Urbana utilizza il concetto di economie di agglomerazione per descrivere le relazioni tra economie interne, esterne e le città. L'autore rileva come i vantaggi legati ai costi e alla qualità sono generati dalla concentrazione in un punto nello spazio di inputs, popolazione ed imprese. Anche se ci sono diverse tassonomie delle economie di agglomerazione, la più nota è quella di Ohlin (1933) poi ripresa da Hoover (1937). Seguendo questa classificazione, possiamo differenziarle tra:

1. economie interne;

2. economie di localizzazione;

3. economie di urbanizzazione;

Le economie interne sono generate allinterno di un'impresa e si possono sottoclassificare, a titolo di esempio, in economie di scala, scopo (od ampiezza), apprendimento e replicazione (Volpato, 1986).

Weber introduce il concetto di agglomerative factor per richiamare i vantaggi relazionati dalla concentrazione di imprese in aree industrializzate. La base del meccanismo di agglomerazione è la riduzione dei costi di trasporto, dove le imprese manifatturiere risultano concentrate in un limitato numero di luoghi. L'obbiettivo è quello di minimizzare i costi di trasporto per le materie prime ed il mercato finale.

L'autore riassume il concetto di economie interne ed esterne nelle brevi righe sottostanti: "An agglomerative factor ... is an advantage or a cheaping of production or marketing which results from the fact that production is carried on to some considerable extent at one place. ... Distinguish in two stages... the first at lower stage is that of the concentration of industry through the simple enlargement of a plant.... the second depends upon the extent of the advantages resulting from a close local association of several plants." (Weber, 1929:126 e ss.).

In un famoso passaggio Ohlin (1933:203) identifica i vantaggi derivanti dalla concentrazione oltre i soli i costi di trasporto e li differenzia in tre categorie: a) economie di concentrazione dell'industria in generale; b) economie esterne di concentrazione di un'industria e c) economie interne:

"There are many other advantages of concentration for the localisation of the industry and the interlocal trade; they are not necessarily relate to differences in transport resources, but might operate anywhere. As however, differences in transport resources tend to draw production to certain places and district, such economies of concentration augment effects. They may be considered under three categories: (1) economies of concentration of industry in general; (2) external economies of concentration of a particular industry, and (3) internal large scale economies of a producing unit...external economies consist partly in the existence of a fully developed market of skilled and specialised labour."

Hoover (1937), richiamando il passaggio precedente propone la nuova divisione delle economie esterne in urbanizzazione e localizzazione che risulta la più nota ed utilizzata nell'ambito dell'Economia Urbana e Regionale:

"In my mind, the greatest deficiency of the Weberian Theory of agglomeration is that it combines three quite distinct influences upon local production costs: (a) Large-scale economies within a firm, consequent upon the enlargement of the firm's scale of production at one point; (b) Localization economies for all the firms in a single industry at a single location, consequent upon the enlargement of the total output 
of that industry at the location; (c) Urbanization economies for all firms in all industries at a single location, consequent upon the enlargement of the total economic size (population, income, output, or wealth) of that location, for all industries taken together" (Hoover, 1937:90).

Camagni (1992:56-57) sviluppa la tassonomia di Hoover (1937) descrivendo le economie di localizzazione e di urbanizzazione.

Le prime, esterne all'impresa ma interne all'industria si suddividono in: a) specializzazione di imprese nel processo produttivo settoriale; riduzione dei costi di transazione in base alla prossimità; b) learning per la manodopera, processo produttivo $\mathrm{e}$ conoscenze; c) servizi specializzati d) atmosfera industriale.

Le seconde, le economie di urbanizzazione, cioè esterne all'industria ma interne all'area analizzata sono suddivise in: a) concentrazione di interventi pubblici o beni pubblici, infrastrutture o economie di scala raggiunte per beni pubblici; b) economie derivanti dalla dimensione urbana in base a mercati di grande dimensione o nicchie di specializzazione c) funzioni terziare superiori, servizi specifici alle imprese, managerialità ed economie di comunicazione ed informazioni dove la città è un incubator di fattori produttivi del mercato del lavoro. Le economie d'urbanizzazione sono legate ai vantaggi che offre la localizzazione in città: possibilità di trovare infrastrutture, scuole, case per i lavoratori, banche, aeroporti ecc. Quando la concentrazione è eccessiva possono avvenire delle diseconomie esterne: crescita dei costi localizzativi e aumento dei costi dei servizi.

\subsubsection{Le economie di rete}

Seguendo l'approccio di Hoover (1937), le economie di agglomerazione hanno due caratteristiche; sono temporalmente e spazialmente statiche. Le economie temporalmente statiche e dinamiche sono state studiate da Glaeser et al. (1992). Le teorie delle esternalità temporalmente dinamiche spiegano simultaneamente come le città nascono e si sviluppano. Le teorie delle esternalità temporalmente statiche sono rappresentate dal concetto delle economie esterne della localizzazione e dell'urbanizzazione, già presentate che spiegano la formazione delle città e la loro specializzazione, ma non la loro crescita (Glaeser et al. 1992:1128) $)^{80}$.

Le economie spazialmente dinamiche sono presenti quando la città è analizzata come un nodo di un sistema di città e non come unità isolata. La generazione di economie esterne relazionate all'interazione tra città, spazialmente dinamiche, è studiata dalle teorie riferite alle reti di città (Pred 1977; Dematteis 1989; Camagni e Salone 1993) e agli spillovers spaziali (Anselin, Varga e Acs 1997 e 2000; Paci e Usai 1999). Tratteremo di questi ultimi nel prossimo paragrafo.

Analizziamo invece la teoria delle reti di città. La teoria centrale del paradigma è che esistono economie e diseconomie generate dall'esistenza di reti di città. Pred (1977) definisce un sistema di città come "un set nazionale e regionale di città che sono interdipendenti in modo che un qualsiasi cambio sostanziale nelle attività economiche, struttura dell'occupazione, reddito nella popolarione di una città membra porterà direttamente od indirettamente delle modificazioni nelle attività economiche, struttura occupazionale e reddito totale o popolazioni di una o più membri del set' (Pred, 1977:13).

\footnotetext{
${ }^{80} \mathrm{Da}$ questo punto di vista possiamo differenziare l'approccio tra economie di localizzazione (temporalmente statiche) e MAR externalities (temporalmente dinamiche) e tra le economie di urbanizzazione (temporalmente statiche) e le economie a là Jacobs (temporalmente dinamiche) ( (Glaeser et al. 1992:1128).
} 
Inoltre l'autore aggiunge come un complesso sistema sociale di città può avere una struttura di interdipendenza e di interrelazioni informative che diventano incredibilmente intricate con il passaggio del tempo.

In riferimento ai distretti industriali Camagni definisce le reti di città e presenta la possibilità dell'esistenza di reti di destinazioni turistiche:

“... è possibile riscoprire a livello della singola città (o del distretto industriale), la funzione della prossimità e la radice dell'agglomerazione nella riduzione dei costi di transazione. Del pari a livello del sistema urbano complessivo, è possibile teorizzare il ruolo della città come nodo di una rete tran-territoriale di rapporti e di transazioni, facilitati dall'esistenza di rete fisiche di trasporto e comunicazione" ... "È possibile a questo punto teorizzare le "reti di citta" come quelle strutture di rapporti e di flussi" fra centri urbani che trovano la loro ragione economica nel conseguimento di economie rispettivamente di complementarietà o di sinergia per $i$ centri urbani che ne fanno parte. ... [esistono due tipi] ... quelle che configurano un processo di divisione spaziale del lavoro ... e le reti di città che svolgono funzioni simili, come le città finanziare, le città direzioni mondiali, le città d'arte riunite in itinerari turistici..." (Camagni, 1992:35 e ss.).

In conclusione possiamo definire le "reti di città" come: "Insiemi di rapporti, orizzontali e non gerarchici, fra centri complementari o similari, rapporti che realizzano la formazione di economie o esternalità rispettivamente di specializzazione/divisione del lavoro $e$ di sinergia/ cooperazione/innovazione" (Camagni, 1992:41).

Camagni, in particolare, descrive i vantaggi della specializzazione e della divisione territoriale del lavoro come realizzabili attraverso economie di integrazione orizzontale fra unità produttive (economie di distretto) e di integrazione verticale attorno a precise filiere di specializzazione. I vantaggi di sinergia, afferma l'autore, si consorziano ai fini di grandi infrastrutture o innovazioni territoriali per le quali i singoli centri non avrebbero né la dimensione di mercato né la necessaria capacità di finanziamento.

Nei lavori di Trullèn e Boix $(2002,2004)$ sono analizzate reti di città nella regione spagnola della Catalogna. Gli autori rilevano reti di città policentriche nell'area metropolitana allargata di Barcellona, dove le componenti della rete sviluppano vantaggi competitivi e vere e proprie economie di rete.

Allo stesso modo si configurano reti di destinazioni turistiche dove i rapporti tra centri complementari e similari realizzano la formazione di esternalità positive di specializzazione e divisione del lavoro.

\subsubsection{Spillovers spaziali}

La parola spillover (spaziale) presuppone un certo tipo di "diffusione" della conoscenza a livello spaziale: "la conoscenza è spesso scambiata senza essere venduta o comprata, la parola spillovers è in questo senso importante" (Rosenthal e Stange, 2002:30). L'idea principale è che "la prossimità geografica faciliti la trasmissione di idee e la diffusione di conoscenza. [...] attraverso l'imitazione, lo spionaggio, e lo scambio di manodopera tra le imprese, le idee sono velocemente sviluppate e diffuse tra le imprese vicine" (Glaeser et al., 1992:1127). I lavoratori sono il principale veicolo della diffusione della conoscenza, appunto degli spillovers.

Gli spillover possono assumere varie forme. La conoscenza può essere incorporata nelle persone che, cambiando occupazione, trasferiscono importanti abilità e informazioni

81 “... si tratta di flussi finalizzati alla transazione economica - che può essere una transazione organizzativa, cooperativa o di mercato a seconda che si svolga rispettivamente all'interno di una gerarchia, come in un'impresa o in un'istituzione, fra $i$ partners di un progetto comune o fra operatori anonimi che effettuano scambia - flussi che in conseguenza banno a che fare con attività di controllo, cooperazione e coordinamento." (Camagni 1992, pp. 35) 
al nuovo datore di lavoro, oppure nei prodotti, come i beni d'investimento o i beni intermedi. Ancora, gli spillover possono trasmettersi attraverso conferenze, stampa specializzata, etc.

Abbiamo discusso fino a questo punto di spillovers di conoscenza. Per spillovers spaziali si può intendere invece sia quelli fisici, come per esempio in Krugman (1991), sia intellettuali o appunto di conoscenza (Glaeser et al., 1992). Gli spillovers tecnologici sono definiti come attività mirate a "condividere $i$ mercati del lavoro, vantaggi da commercio tra imprese inter-penetrate, effetti di conoscenza locale sugli spin-offs d'imprese, e tutte le altre forze che influenzano un'impresa nel localizzarsi vicino ad un'altra impresa della stessa industria" (ibidem: 894) ${ }^{82}$.

Sono tre le teorie principali relative agli spillovers (Glaeser et al., 1992:1130 e ss.). La teoria MAR, la teoria della diversità e la teoria della competitività à la Porter.

La teoria MAR (Marshall-Arrow-Romer) si incentra sugli spillovers in una specifica industria dovuti alla concentrazione delle imprese ed alle economie di localizzazione.

Questa proviene dalla specializzazione industriale, le sue origini sono nella teoria economica tradizionale. Romer $(1986,1990)$ sviluppa l'argomento della specializzazione partendo ancora da Marshall. Questo sviluppo, riferito poi ad Arrow (1962) e alla teoria della crescita, ha prodotto la teoria denominata MAR (Marshall-Arrow-Romer) (Glaeser et al. 1992). La teoria MAR argomenta che gli spillovers (esternalità) comuni alla stessa industria, che derivano dall'agglomerazione geografica, hanno un ruolo fondamentale nella crescita delle imprese. Le imprese che sono vicine si avvantaggiano maggiormente da conoscenze relative allo stesso settore di specializzazione che da quelle di altri settori ${ }^{83}$.

Porter (1990, 1998, 2000) aggiunge alla concentrazione industriale il ruolo nella rivalità dell'industria. Questo fattore spinge le imprese a competere più intensamente ed a sviluppare innovazioni su quelle altrui. Porter argomenta che due specifici fattori: la competizione locale e la concentrazione (geografica) dell'industria sono le maggiori origini del successo di un sistema locale. La competizione locale promuove il dinamismo dell'intero sistema e la concentrazione spaziale aumenta l'interazione degli attori. ${ }^{84}$.

Il terzo filone di pensiero è quello proveniente dagli studi di Jacobs basato sulla diversità. In questo studio si rileva che il maggior ruolo svolto dagli spillovers è di generare una fertilizzazione incrociata tra imprese di industrie diverse. Le economie di diversità presuppongono che i maggior benefici per una città sia dovuto alla sua diversificazione. Jacobs (1969) rileva come questa fonte di vantaggi legati alla diversità in una città $\mathrm{o}$ in una regione sia uno dei principale fattore di crescita: "People who thinks of cities simply as towns that

${ }^{82}$ L'unico lavoro che approfondisce il problema degli spillovers nell'ambito turistico è il lavoro di Deidda, Paci ed Usai (2002). Nel contributo gli autori evidenziano come la crescita dell'occupazione suddivisa per settore (codice Ateco 91) nei 784 sistemi locali definiti dal algoritmo ISTAT-Sforzi del 1996, sia dovuta non solo alle caratteristiche strutturali del singolo sistema locale del lavoro, ma dipenda anche dai sistemi limitrofi. In particolare, considerando la voce H "Hotel e Ristoranti" si nota un'alta relazione geografica tra la crescita di un sistema locale (in questo settore) e gli altri vicini. In altre parole, la crescita di un sistema locale nel comparto degli Hotel e Ristoranti influenza anche la crescita dei sistemi locali vicini. Esiste, da questo punto di vista, una sorta di rete di sistemi locali.

${ }^{83}$ In questo modo in Glaeser et al. (1992) non si individua una relazione positiva tra concentrazione dell'industria e crescita, ma si trova che la diversità dell'industria e la competizione sono i principali fattori che promuovono la crescita.

84 In "The competitive advantage of nations" (1990) Porter sviluppa un framework per analizzare i vantaggi competitivi delle diverse economie nazionali. Il diamante è costituito da quattro componenti che trattano le fonti del vantaggio competitivo:a) condizione dei fattori, b) contesto di strategia e concorrenza delle imprese, c) condizioni della domanda, d) industrie collegate e di supporto. In particolare, si parla dei fattori come beni tangibili, informazioni, sistema giuridico, risorse naturali, umane, di capitali, ecc. Il contesto della strategia riassunto come l'insieme di regole, incentivi che governano la rivalità locale. La domanda analizzata come le caratteristiche ed esigenze. Le industrie collegate considerate come l'insieme di fornitori e collaboratori. 
have kept growing larger, are believers in a preformation theory of city growth, an enlargment of what is essentially already there. I am arguing, rather, an epigenisis theory of cities: the idea that a city grows by a process of gradual diversification and differentation of its economiy" (Jacobs 1969: 126).

Si rinvia a Griliches (1979) per un'ampia rassegna empirica sul ruolo degli spillovers.

\subsection{UNA PROPOSTA DI CLASSIFICAZIONE}

Nella figura 3.1 si presenta una classificazione delle economie esterne passate in rassegna nel corso di questo capitolo. In particolare si propone una distinzione in base alla provenienza dei vantaggi di localizzazione: interne o esterne allimpresa (network di imprese), interne o esterne al sistema locale (network di sistemi locali) ed infine interne od esterne ad una specifica industria.

Figura 3.1: La classificazione delle economie esterne e spillovers;

\begin{tabular}{|c|c|c|c|c|c|}
\hline & \multicolumn{2}{|c|}{ Interno allimpresa } & \multicolumn{2}{|c|}{ Esterno all'impresa } & \\
\hline & $\begin{array}{l}\text { Interno } \\
\text { all'impresa }\end{array}$ & $\begin{array}{l}\text { Esterno } \\
\text { all'impresa }\end{array}$ & $\begin{array}{l}\text { Interno } \\
\text { all'industria }\end{array}$ & $\begin{array}{l}\text { Esterno } \\
\text { all'industria }\end{array}$ & \\
\hline $\begin{array}{l}\text { Interno al } \\
\text { sistema } \\
\text { locale }\end{array}$ & $\begin{array}{l}\text { Economie } \\
\text { Interne }\end{array}$ & & $\begin{array}{l}\text { Economie di } \\
\text { localizzazione }\end{array}$ & $\begin{array}{l}\text { Economie di } \\
\text { urbanizzazione }\end{array}$ & $\begin{array}{l}\text { Externalities a } \\
\text { la Hoovers }\end{array}$ \\
\hline \multirow[t]{2}{*}{$\begin{array}{l}\text { Esterno al } \\
\text { sistema } \\
\text { locale }\end{array}$} & & & $\begin{array}{l}\text { erne di rete d } \\
\text { Spillovers }\end{array}$ & & $\begin{array}{l}\text { Studi tramite la } \\
\text { spatial statistics }\end{array}$ \\
\hline & & $\begin{array}{l}\text { Reti di } \\
\text { imprese }\end{array}$ & $\begin{array}{l}\text { Distretto } \\
\text { industriale a la } \\
\text { Marshall }\end{array}$ & & \\
\hline
\end{tabular}

Fonte: nostra elaborazione da Boix, 2004.

Si prospettano quindi tre tipi di economie esterne su cui ci focalizzeremo nella parte empirica al fine di misurare la competitività dei sistemi locali turistici individuati:

a) le economie esterne all'impresa, ma interne al sistema locale ed ad un'industria specifica (economie di localizzazione);

b) le economie esterne all'impresa ed interne al sistema locale, ma trasversali a più industrie (economie di urbanizzazione);

c) le economie esterne all'impresa ed al sistema locale che possono però essere sia interne che esterne ad un'industria specifica e che si sviluppano in rete di sistemi locali (economie di networks). 


\section{CAPitolo IV}

\section{LA METODOLOGIA UTILIZZATA: L'ECONOMETRIA SPAZIALE}

\subsection{PRemessa}

L'Econometria Spaziale è quella metodologia di analisi statistica che cerca di misurare patterns di osservazioni geograficamente collegate. Questa tecnica può essere fatta risalire ai due principali lavori di Paelink e Klaasen (1979) e di Anselin (1988) e vi viene definita come:

- "Set of methods that deals with the explicit treatment of space in multiregional models" (Paelink e Klaasen, 1979)

- "Collection of techniques that deals with the peculiarities caused by space in the statistical analysis of regional science models" (Anselin, 1988)

L'econometria spaziale è quindi prima di tutto un toolkit di tecniche e metodi utili al ricercatore che cerca soluzioni a problemi e difficoltà nel trattare dati geo-referenziati soggetti ad un eventuale interazione nello spazio. Questa disciplina cerca di risolvere basicamente due ordini di problematiche: la dipendenza spaziale e l'eterogeneità spaziale. Il primo, denominato appunto spatial dependence, riguarda due aspetti: l'interazione spaziale delle unità osservate e la auto-correlazione spaziale negli errori del modello econometrico.

Nel prossimo paragrafo cercheremo di spiegare brevemente la necessità di questa tecnica specifica per il trattamento di dati territoriali. In breve descriveremo i due problemi principali che questa tecnica cerca di risolvere. Le pagine successive di questo capitolo saranno invece dedicate alla descrizione dei modelli fondamentali della Spatial Econometrics utile per la stima delle economie esterne dei sistemi locali turistici nella seconda parte di questo lavoro.

Nella figura sottostante si riportano, a titolo di esempio, le stime sulla criminalità nei confini municipali della città di Colombus effettuate da Anselin (1988). L'autore descrive come i patterns dei crimini siano diversi se si considera l'influenza che i quartieri con alta criminalità hanno su quelli limitrofi (da sinistra a destra).

Figura 4.1: Esempi di Spatial dependence;

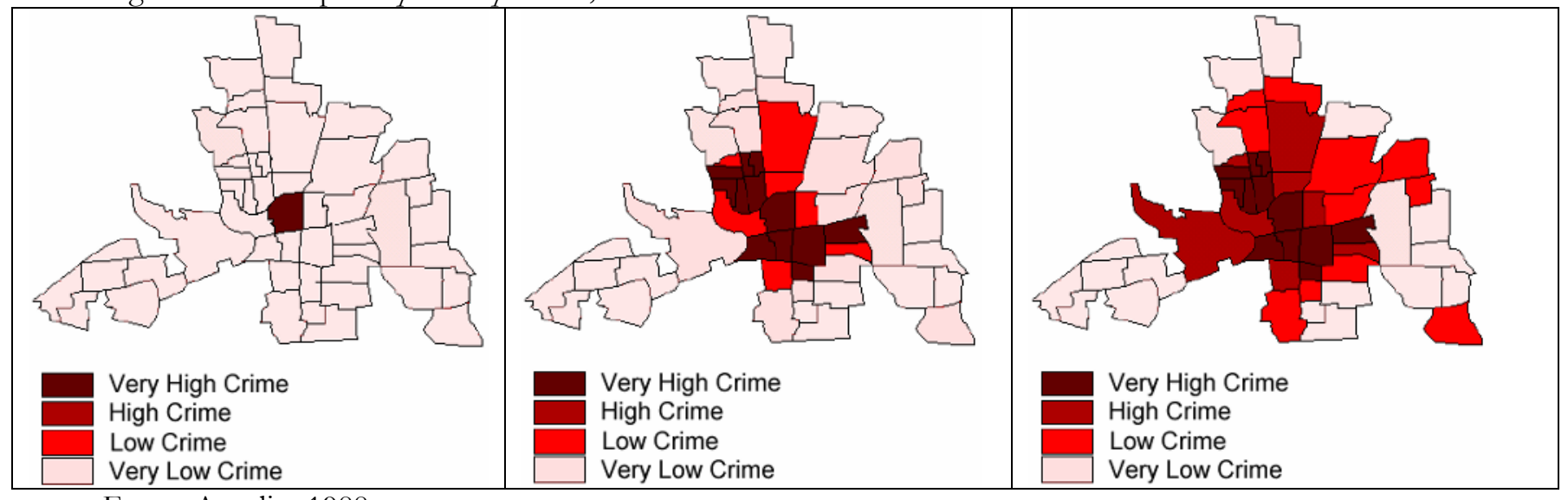

Fonte: Anselin, 1988; 
Un altro esempio concreto di dipendenza spaziale è analizzato da Le Sage (1999). Durante il 1981 le cavallette si nutrirono di circa 12 milioni di acri di foresta in 17 stati americani del nord. Successivamente la diffusione si allargò agli stati del mid-west e del pacifico. Nel 1992 per esempio si stima in Michingan che più di 700.000 acri di foresta registrassero un tasso di desfogliamento del 50\%. La figura 4.2 mostra la distribuzione spaziale delle falene nel Michigan del sud nel 1991. Il più alto livello di falene si registra nell'area bianca nel centro della figura. Muovendosi verso l'esterno della distribuzione assume la forma di cerchi concentrici di diffusione, anche perché le falene non possono volare per lunghi tratti quindi la loro diffusione è territoriale ${ }^{85}$.

Figura 4.2: Propagazione delle Falene nello stato del Michigan.

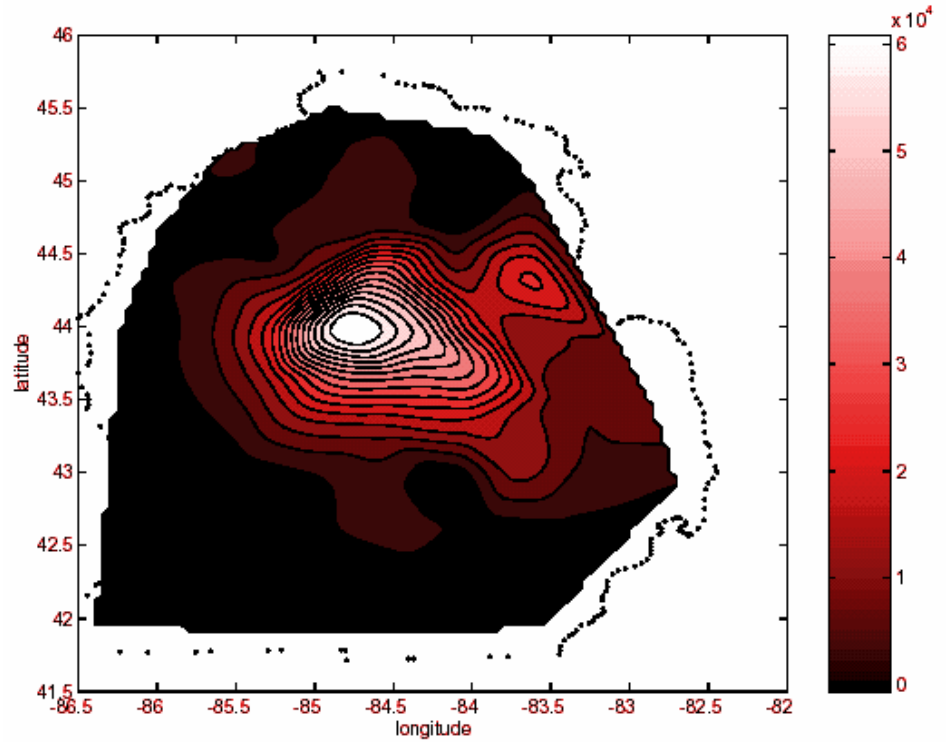

Fonte: Le Sage, 1999;

L'autore analizza la propagazione delle falene in Michigan, presentando questo caso come un classico esempio di un fenomeno naturale di dipendenza spaziale.

\subsection{LA DIPENDENZA SPAZIALE E L'ETEROGENEITÀ}

La Spatial dependance si registra quando l'osservazione nel luogo $i$ dipende da altre osservazioni in luoghi diversi $j$ con $i \neq j$. Esistono due tipi di spatial dependance: quando un'osservazione influenza un altro individuo e l'auto-correlazione spaziale, che si riferisce all'influenza stocastica causata nella misurazione degli errori. La correlazione negli errori si registra quando il loro valore nella stima di un'osservazione è connesso a quello di un'altra osservazione. In altre parole, i residui si distribuiscono tramite patterns spaziali.

Che cosa è invece la spatial heterogeneity? L'eterogeneità spaziale si riferisce a quel fenomeno in cui le osservazioni (spatial units) non sono omogenee nello spazio. Esistono due tipi di eterogeneità spaziale: l'instabilità strutturale e l'eteroschedasticità. Si registra instabilità strutturale quando le osservazioni non sono omogenee nello spazio (per esempio modelli nord-sud), mentre l'eteroschedasticità è causata dalla presenza di outliers.

\footnotetext{
${ }^{85}$ Un set di punti presi dallo stesso anello dimostra un'alta correlazione con gli altri. Stesse osservazione nello stesso anello sono altamente correlate, mentre il tasso di correlazione diminuirebbe prendendo in considerazione osservazioni da anelli più distanti (Le Sage, 1999:5).
} 
La metodologia utilizzata: l'Econometria Spaziale

Il problema più importante è la spatial dependance, infatti la spatial heterogeneity può essere risolta tramite tecniche econometriche convenzionali ${ }^{86}$; quando invece le osservazioni sono correlate spazialmente le convenzionali tecniche econometriche possono registrare risultati erronei (Anselin 1988). Riferendoci ad un generico modello di massima verosimiglianza possiamo elencare le condizioni in cui questo modello risulta un modello di

stima robusto:

$$
Y=X B+\varepsilon
$$

$$
\varepsilon \sim \mathrm{N}\left(\mu, \sigma^{2} I\right)
$$

Linearità del modello $\rightarrow y=a+b X$;

Normalità dei residui $\rightarrow \varepsilon \sim N\left(0, \sigma^{2}\right)$;

Errore standard con media zero $\rightarrow E[\varepsilon]=0$;

Gli errori standard sono incorrelati ed hanno varianza costante $\rightarrow E\left[\varepsilon \varepsilon^{\prime}\right]=\sigma^{2} I$.

Tutto questo si complica se c'è un certo tipo di dipendenza spaziale. Infatti questa è una situazione nella quale la variabile dipendente o l'errore stimato di ogni luogo è correlato con l'osservazione o il valore dell'errore di altri luoghi: "the situation where the dependent variable or error term at each location is correlated with observations on the dependent variable or values for the error term at other locations" (Anselin 1992):

cioè risulta:

$$
E\left[y_{i} y_{j}\right] \neq 0
$$

oppure:

$E\left[\varepsilon_{i} \varepsilon_{j}\right] \neq 0$

Quindi possono risultare due problemi: se l'errore è dovuto ad interazione spaziale (spatial interaction), lo stimatore può essere inefficiente (biased), cioè può contenere un errore dovuto a variabili esplicative omesse. D'altro canto se si presenta un caso di autocorrelazione spaziale (spatial autocorrelation) negli errori i parametri sono corretti, ma il test può essere inefficiente. Inoltre, non possiamo sapere in quale di queste due situazioni ci troviamo. L'econometria spaziale ci è d'aiuto in questa situazione tramite due soluzioni: patterns territoriali di contatto e un gruppo di stimatori efficaci per questo tipo di problemi.

La prima soluzione è la matrice dei contatti o matrix of spatial contacts (W). Nella figura 4.1 sono presentate 5 osservazioni (territoriali) che risultano essere in contatto tramite patterns spaziali. La prima osservazione è in contatto reciproco con la seconda; questa è in contatto con la quarta e così via. Nella parte destra si descrive la rispettiva matrice $\mathbf{W}$ dei contatti in base ai patterns spaziali descritti. La matrice dei contatti W definisce i contatti di ogni elemento $i$ (riga) con ogni elemento $j$ (colonna). Naturalmente ogni unità è in contatto con se stessa, quindi gli elementi della diagonale sono per costruzione nulli.

\footnotetext{
86 Alcune procedure specifiche come vedremo in seguito sono state sviluppate per risolvere questi problemi
} specificatamente nello spazio (Anselin 1988). 
Tabella 4.1: Patterns spaziali e Matrice dei contatti;

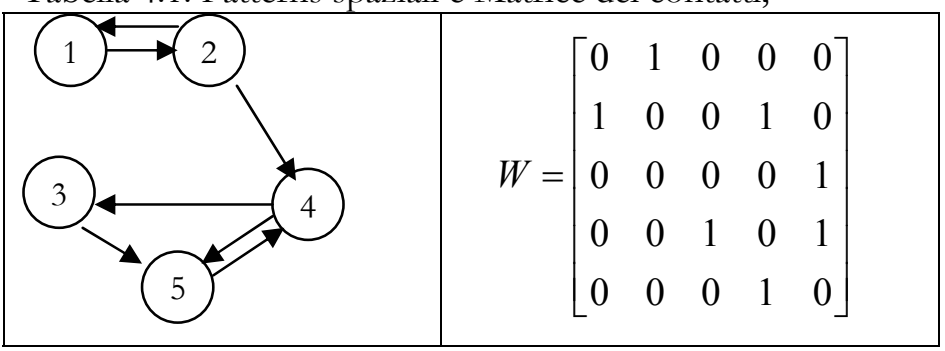

Fonte: Boix, (2003);

La matrice dei contatti $\mathbf{W}$ può inoltre essere anche "pesata" in base alla quantità di contatti di ogni osservazione con un'altra (import-export, lavoratori in-out, bilancia dei pagamenti, etc.). La matrice si modifica nel modo seguente:

Tabella 4.2: Patterns spaziali e Matrice dei contatti;

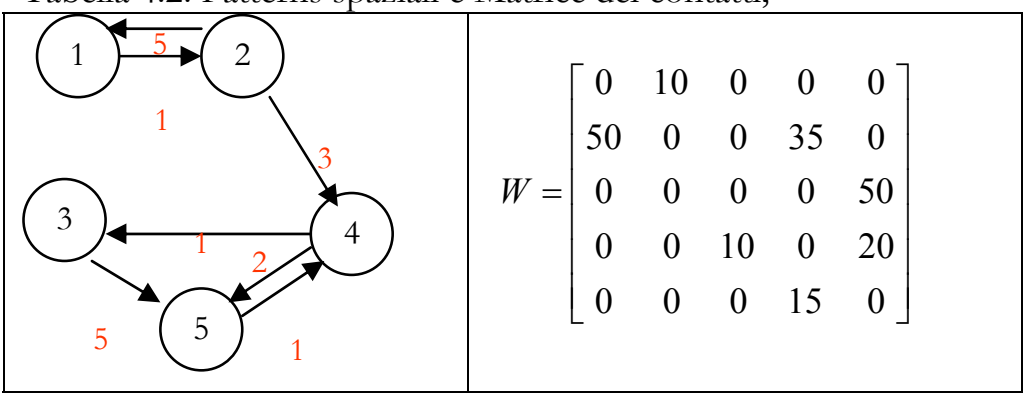

Fonte: Boix, (2003);

Il secondo problema introdotto è la necessità di utilizzare alcuni test per misurare la dipendenza spaziale. I test (univariati) della econometria spaziale sono test che misurano la dipendenza spaziale di una variabile in base a dei patterns spaziali (matrice dei contatti $\mathbf{W}$ ).

Tabella 4.3: Tests univariati per la dipendenza spaziale;

\begin{tabular}{|l|c|}
\hline \multicolumn{2}{|c|}{ Test univariati per la dipendenza spaziale } \\
\hline Dipendenza Globale & Dipendenza Locale \\
\hline - I di Moran & - I di Moran Locale \\
- C di Geary & - Gi e Gi* \\
- G Statistica & - Nuovi Gi e Gi* \\
\hline
\end{tabular}

Fonte: Moreno, Vaya, (2000);

I test multivariati misurano invece la dipendenza spaziale in un modello di regressione multivariato (variabile esplicativa e variabili dipendenti):

Tabella 4.4: Tests univariati per la dipendenza spaziale;

\begin{tabular}{|l|l|}
\hline \multicolumn{2}{|c|}{ Dipendenza spaziale in un modello di regressione } \\
\hline Interazione spaziale & \multicolumn{2}{|c|}{ Autocorralazione spaziale degli errori } \\
\hline$-\quad$ LM lag & - I di Moran sull'errore \\
$-\quad$ LM lag robusto & - LM errore \\
& - LM errore robusto \\
& - KR erroe \\
\hline
\end{tabular}

Fonte: Moreno, Vaya, (2000); 
La metodologia utilizzata: l'Econometria Spaziale

Descriveremo nelle prossime pagine i test univariati e i modelli multivariati per misurare la correlazione spaziale. Infine nella tabella sottostante elenchiamo i software disponibili per le analisi statistiche spaziali, descrivendone brevemente le principali caratteristiche.

Tabella 4.5: I Softwares per le analisi di Econometria Spaziale;

\begin{tabular}{|c|c|}
\hline $\begin{array}{l}\text { Pacchetti Standard che non sviluppano funzioni } \\
\text { di econometria spaziale }\end{array}$ & "E-Views", "SPSS“, “SAS”, etc. \\
\hline $\begin{array}{l}\text { Packages che hanno solo alcuni indicatori, ma } \\
\text { non hanno modelli multivariati; }\end{array}$ & "S-Plus" \\
\hline $\begin{array}{l}\text { Pacchetti specifici che sviluppano modelli di } \\
\text { Econometria spaziale; }\end{array}$ & $\begin{array}{l}\text { "SpaceStat 1.91" (Anselin, 1992) e "GeoDa" } \\
\text { (Anselin 2003) } \\
\text { "Econometric toolbox for Matlab 6.x" (J. } \\
\text { LeSage; free software) } \\
\text { "K. Konis" della Insightful Co. }\end{array}$ \\
\hline
\end{tabular}

Fonte: nostra elaborazione;

\subsection{AlCUNi CENNI STORICI SULl'ECONOMETRIA SPAZIALE}

Senza dubbio lo sviluppo dell'econometria spaziale è iniziato con i primi indici specifici per individuare e misurare la presenza di auto-correlazione spaziale. I lavori di Moran (1948) e di Geary (1954) sono tuttora fondamentali per questo tipo di analisi. Dopo questa prima fase di riconoscimento del problema, negli anni settanta, si inizia a parlare del termine Econometria Spariale, riferito principalmente ai lavori di Paelink e Klaassen (1979) e allo sforzo di misurare la auto-correlazione spaziale nei termini di perturbazione per una stima di regressione (Moreno, Vayà, 2000). Solo negli anni ottanta e con il noto lavoro di Anselin (1988) si arriva finalmente all'Econometria spaziale moderna. Anselin (1988) definisce tale disciplina come:

"the collection of tecniques that deal with the pecularities caused by space in the statistical analysis of regional science models is considered to be the domain of spatial econometrics" (Anselin 1988:6) ${ }^{87}$.

Il termine Spatial econometric, come già detto, fu coniato da Jean Paelinck nei primi annni settanta nell'analizzare una nuova branca che trattava la stima di specifici test incontrati nell'implementazione di modelli econometrici multiregionali ${ }^{88}$. In Anselin e Bera (1998) si aggiunge la regione, la localizzazione, l'interazione spaziale formando la metodologia di analisi dell'Economia Regionale ed Urbana. Il grande sviluppo si ha negli anni ottanta con i primi lavori specifici territoriali. Il rinnovato interesse per il ruolo del territorio e dei localismi nelle scienze sociali, la possibilità di aver a disposizione estese basi di dati georeferenziati e lo sviluppo di software applicativi geografici come i Sistemi Informativi Geografici (GIS) come Arview, sono state alcune delle cause che hanno permesso lo sviluppo di questa disciplina. Inoltre lo sviluppo da parte di Anselin di un software applicativo destinato specificatamente a questo tipo di analisi con test e modelli per analisi spaziali, il noto SpaceStat (Anselin 1992) ha facilitato l'applicazione di questi metodi favorendo la crescita dei contributi. Nella tabella sottostante si presentano gli studi sviluppati tramite l'Econometria spaziale fino al 2000. In particolare si vuole sottolineare la multidisciplinarietà di questa tecnica.

87 "la collezione di tecnica che tratta le pecularietà causate dallo spazio nell'analisi statistica dei modelli tradizionali della scienza regionale."

88 In particolare nel loro libro Spatial Econometric, Paelink e Klassen (1979) sottolineavano cinque caratteristiche di problemi: 1) il ruolo della interdipendenza spaziale nei modelli spaziali; 2) l'asimmetria nelle relazioni spaziali; 3) l'importanza di fattori esplicativi localizzati in luoghi diversi; 4) la diversità tra interazione ex-post e ex-ante 5) modelli espliciti che consideravano lo spazio. 
Tabella 4.6: Principali applicazioni empiriche della Econometria spaziale, 1990-1999;

\begin{tabular}{|c|c|c|c|}
\hline Autori & Anno & Rivista & Soggetto \\
\hline Nagaraj, R. et al & 1999 & $\begin{array}{l}\text { Annales d'Economie et de } \\
\text { Statistique }\end{array}$ & $\begin{array}{l}\text { Crescita economica ed esternalità } \\
\text { spaziale }\end{array}$ \\
\hline Lopez-Bazo, E. et al & 1999 & Annals of Regional Science & $\begin{array}{l}\text { Dinamiche regionale e } \\
\text { convergenza }\end{array}$ \\
\hline Rey, S. e B.D. Montouri & 1999 & Regional Studies & Crescita e convergenza regionale \\
\hline Mencken, F.C. & 1998 & Growth and Change & $\begin{array}{l}\text { Crescita economica e differenze } \\
\text { regionali }\end{array}$ \\
\hline Vandeveer, L. et al & 1998 & $\begin{array}{l}\text { Review of Agricultural } \\
\text { Economics }\end{array}$ & Mercati rurali e GIS \\
\hline $\begin{array}{l}\text { Rietveld, P. e } \\
\text { Wintershoven, P. }\end{array}$ & 1998 & Regional Science & $\begin{array}{l}\text { Red de infraestructuras en } \\
\text { regiones frontera }\end{array}$ \\
\hline Basu, S. e Thibodeau, T. & 1998 & $\begin{array}{l}\text { Journal of Real Estate } \\
\text { Finance and Economics }\end{array}$ & $\begin{array}{l}\text { Determinanti del prezzo delle } \\
\text { abitazioni }\end{array}$ \\
\hline Macedo, P.B.R. & 1998 & $\begin{array}{l}\text { Revista Brasileira ce } \\
\text { Economia }\end{array}$ & $\begin{array}{l}\text { Determinanti del prezzo delle } \\
\text { abitazioni }\end{array}$ \\
\hline Moreno, R. et al & 1997 & $\begin{array}{l}\text { International Journal of } \\
\text { development planning } \\
\text { literature }\end{array}$ & Crescita e capitale pubblico \\
\hline $\begin{array}{l}\text { Bivand, R. e Szmanski, } \\
\text { S. }\end{array}$ & 1997 & Economic Letters & Competizione locale e esternalità \\
\hline $\begin{array}{l}\text { Ashworth, J. E Parker, } \\
\text { S.C. }\end{array}$ & 1997 & $\begin{array}{l}\text { Scottish journal of political } \\
\text { economy }\end{array}$ & $\begin{array}{l}\text { Determinanti del prezzo delle } \\
\text { abitazioni }\end{array}$ \\
\hline Aten, B. & 1997 & $\begin{array}{l}\text { International Regional } \\
\text { Science Review }\end{array}$ & Prezzi e commercio internazionale \\
\hline Can A. e Megbolugbe, I. & 1997 & $\begin{array}{l}\text { Journal of Real Estate } \\
\text { Finance and Economics }\end{array}$ & Indice dei prezzi delle abitazioni \\
\hline $\begin{array}{l}\text { Kelejian, H. e Robinson, } \\
\text { D. }\end{array}$ & 1997 & Regional Science & $\begin{array}{l}\text { Studio della produttività regionale } \\
\text { ed infrastrutture pubbliche }\end{array}$ \\
\hline Anselin, L. et al. & 1997 & Journal of Urban economics & Spillovers tecnologici \\
\hline $\begin{array}{l}\text { O’Luoghilin, J. e } \\
\text { Anselin, L. }\end{array}$ & 1996 & Economic Geography & Commercio internazionale \\
\hline Bernat, G. & 1996 & Journal of Regional Science & Crescita regionale \\
\hline Aten, B. & 1996 & $\begin{array}{l}\text { Review of Incombe and } \\
\text { Wealth }\end{array}$ & Prezzi e commercio internazionale \\
\hline Lyson, T. e Tolbert, C. & 1996 & Environment and Planning & $\begin{array}{l}\text { Dimensione della impresa e } \\
\text { benessere locale }\end{array}$ \\
\hline Meen, G. & 1996 & Housing Studies & $\begin{array}{l}\text { Prezzo di abitazione nel mercato } \\
\text { nazionale e locale }\end{array}$ \\
\hline Barkley, D. et al. & 1995 & Regional Science & Effetti spread e backwash \\
\hline Molho, I. & 1995 & Journal of Regional Science & Disoccupazione e esternalità \\
\hline $\begin{array}{l}\text { Benirschka, M. e } \\
\text { Brinkley, J.K. }\end{array}$ & 1994 & $\begin{array}{l}\text { American Journal pf } \\
\text { Agricolture economics }\end{array}$ & $\begin{array}{l}\text { Studio del valore della terra } \\
\text { agricola }\end{array}$ \\
\hline Holtz-Eakin, D. & 1994 & $\begin{array}{l}\text { Review of Economics and } \\
\text { Statistics }\end{array}$ & Spillovers e infrastruttura pubblica \\
\hline Case, A.C., et al & 1993 & Journal of public economics & Spillovers fiscali \\
\hline Murdoch, J. et al. & 1993 & Public Finance quarterly & Spesa pubblica \\
\hline Le Sage, J.P. & 1993 & $\begin{array}{l}\text { American journal of } \\
\text { Agricolture economics }\end{array}$ & Prezzi dei mercati agricoli \\
\hline Case A.C. & 1992 & $\begin{array}{l}\text { Regional science and urban } \\
\text { economics }\end{array}$ & $\begin{array}{l}\text { Adozione della nuova tecnologia } \\
\text { in agicoltura }\end{array}$ \\
\hline Dubin, R.A: & 1992 & $\begin{array}{l}\text { Regional Science and Urban } \\
\text { Economics }\end{array}$ & $\begin{array}{l}\text { Qualità locale e prezzo delle } \\
\text { abitazioni }\end{array}$ \\
\hline $\begin{array}{l}\text { Heikkila, E. e } \\
\text { Kantiotou, C. }\end{array}$ & 1992 & $\begin{array}{l}\text { Regional Science and Urban } \\
\text { Economics }\end{array}$ & $\begin{array}{l}\text { Impatto fiscale nel cambio di uso } \\
\text { della terra }\end{array}$ \\
\hline Congdon, P. & 1990 & Urban Studies & Tasso di mortalità e spillovers \\
\hline Can, A. & 1990 & Economic Greography & Determinanti prezzi abitazioni \\
\hline
\end{tabular}

Fonte: Estratto da Moreno e Vayà, 2000; 
La metodologia utilizzata: l'Econometria Spaziale

Nonostante questa prima divulgazione, tuttora rimane una scarsa diffusione a livello didattico e sono pochi i riferimenti generali nei testi di statistica generale (Moreno e Vayà 2000).

\subsection{TEST DI EFFETTI SPAZIALI}

Il termine eterogeneità spaziale come già detto, si riferisce alla variazione di relazioni nello spazio. Abbiamo già detto come questa si può suddividere sotto due aspetti: l'instabilità strutturale e l'eteroschedasticità. Il primo caso emerge quando la variabile sotto studio manca di stabilità e robustezza nello spazio. In altre parole i parametri stimati possono variare sensibilmente in diversi luoghi quando la stima non è omogenea nel territorio (Anselin, 1988). Questo fenomeno come già detto può emergere quando si misurano, con uno stesso modello, realtà diverse territorialmente, come il nord e il sud di un paese o di zone centro/periferia. Il secondo fenomeno si registra quando a causa di omissioni di variabili o del verificarsi di errori con patterns spaziali.

La dipendenza spaziale è una conseguenza dell'esistenza di una relazione funzionale tra ciò che succede in un luogo e quello si verifica in un altro. In altre parole il valore di una variabile in un luogo, sistema locale o regione, dipende non solo da quello che succede in quel luogo, ma anche da quello che succede nei luoghi, regioni ed i sistemi locali vicini. Tale influenza può quindi essere positiva o negativa se i risultati delle regioni vicine influenzano positivamente o negativamente una variabile stimata. Sono due i fenomeni di dipendenza spaziale: l'esistenza di errori di misurazione e i fenomeni di interazione spaziale.

Gli errori di misurazione possono emergere quando vi è una scarsa coincidenza tra l'estensione spaziale del fenomeno sotto analisi e l'unità territoriale di osservazione. In altre parole l'utilizzo di unità di analisi territoriale per osservare un evento prescinde da tali confini (amministrativi, comunali etc.). L'econometria spaziale risolve questo problema inserendo nella stima dei test i patterns spaziali di contatto, tramite la matrice di contatto $\mathbf{W}$, presentata nelle pagine precedenti, e sviluppando dei test che utilizzano la matrice $\mathbf{W}$ per stimare la dipendenza spaziale. Passiamo in rassegna nella parte seguente $i$ test univariati per questo tipo di analisi.

\subsubsection{I test univariati spaziali locali e globali}

Lo scopo di questo paragrafo è di analizzare i test univariati per la misurazione della dipendenza spaziale. In altre parole constatare se la variabile di studio è distribuita aleatoriamente nello spazio o se esiste un'associazione significativa di valori nelle regioni limitrofe.

Come già presentato esistono alcuni test sviluppati specificatamente a tal fine: la I di Moran, la C di Geary e la G(d) di Getis y Ord. Il test I di Moran (Moran 1948) è rappresentato dalla seguente formula:

$$
I=\frac{N}{S_{o}} \frac{\sum_{i j}^{n} W_{i j}\left(x_{i}-\bar{x}\right)\left(x_{j}-\bar{x}\right)_{i j}}{\sum_{i=1}^{n}\left(x_{i}-\bar{x}\right)^{2}} i \neq j
$$


dove $x_{i}$ è la variabile quantitativa $x$ nell'unità territoriali $i, \bar{x}$ è la sua media delle osservazioni, $w_{i j}$ sono i pesi della matrice $\mathrm{W}, \mathrm{N}$ e il numero delle osservazioni e So è la somma di tutti i pesi cioè la sommatoria per righe e colonne della matrice dei pesi $\mathrm{W}^{89}$.

Per un numero di osservazioni molto grande la $I$ di Moran standardizzata si distribuisce come una normale $\mathrm{N}(0,1)$, in questo caso un valore non significativo porterà a rifiutare la ipotesi nulla di non dipendenza spaziale, mentre un valore significativo (positivo $\mathrm{o}$ negativo) ci informerà sulla direzione di questa dipendenza (positiva o negativa). In altre parole se la $I$ di Moran è positiva (negativa) e significativa dimostra una dipendenza spaziale positiva (negativa) tra regioni vicine della variabile studiata ${ }^{90}$.

Un secondo indice di dipendenza spaziale è la C di Geary (1954) che ha la seguente espressione:

$$
C=\frac{N-1}{2 S_{o}} \frac{\sum_{i j}^{N} W_{i j}\left(x_{i}-x_{j}\right)}{\sum_{i=1}^{N}\left(x_{i}-\bar{x}\right)^{2}} i \neq j
$$

dove i simboli utilizzati seguono la spiegazione della I di Moran. Come la I di Moran, la $C$ di Geary standardizzata si distribuisce come una normale $\mathrm{N}(0,1)$, in questo caso un valore non significativo porterà a rifiutare la ipotesi nulla di non dipendenza spaziale, mentre un valore significativo (positivo o negativo) ci informerà sulla direzione di questa dipendenza (negativa o positiva, quindi inversa a differenza della $I$ di Moran). In altre parole se la $C$ di Geary è negativa (positiva) e significativa dimostra una dipendenza spaziale positiva (negativa) della variabile sotto studio in regioni vicine.

Il terzo test che presentiamo, il test $G(d)$, definito recentemente da Getis e Ord (1992) come una misura della concentrazione spaziale di una variabile e si presenta nella seguente forma:

$$
G(d)=\frac{\sum_{i=1}^{N} \sum_{j=1}^{N}{ }_{W_{i j}}(d) x_{i} x_{i}}{\sum_{i=1}^{N} \sum_{j=1}^{N} x_{i} x_{i}} i \neq j
$$

dove le unità territoriali analizzate sono considerate in base ad una distanza $d$ moltiplicata per la matrice di pesi. In altre parole, a differenza degli altri modelli, la matrice W è ponderata per una distanza $d$. La standardizzata della $G(d)$ si distribuisce asintoticamente come una normale $\mathrm{N}(0,1)$. L'interpretazione è simile a quella degli altri test precedenti, a differenza di un valore positivo (negativo) e significativo indica una tendenza alla concentrazione di valori elevati di x nello spazio analizzato. Questi test possono essere utilizzati in forma congiunta per stimare diverse caratteristiche della dipendenza spaziale.

\footnotetext{
${ }^{89}$ In particolare quando una matrice è standardizzata per righe il fattore $\mathrm{S}$ è uguale ad $\mathrm{N}$, dato che la somma di ogni riga è uguale a 1 . In questo caso l'indice si approssima ad identificare una varianzia nello spazio (Anselin 1992).

${ }^{90}$ Lo scopo di questo paragrafo non risulta quello di approfondire le dimostrazioni statistiche dei test e dei modelli econometrici, ma presentare un panorama generale che si a di aiuto al lettore per l'analisi quantitativa effettuata nella seconda parte di questo scritto, per l'eventuale dimostrazione si rimanda ad Anselin (1988) in riferimento al questo test ed agli altri che verranno presentati in questo paragrafo.
} 
Per ulteriori approfondimenti si rimanda a Moreno e Vayà $(2000)^{91}$. Inoltre, mentre dal $G(d)$ è una misura della concentrazione nello spazio la I di Moran è un una misura di correlazione di ogni unità territoriale presa in considerazione con le altre.

L'ultimo test di dipendenza spaziale che presentiamo è lo scatterplot di Moran. Questo grafico indica, nell'asse delle ascisse le osservazioni della variabile $x$ standardizzata e nell'asse $y$, il ritardo spaziale ottenuto moltiplicando la variabile $x$ per la matrice $W$. I valori sono poi normalizzati.

Figura 4.3: Scatterplot di Moran (univariate).

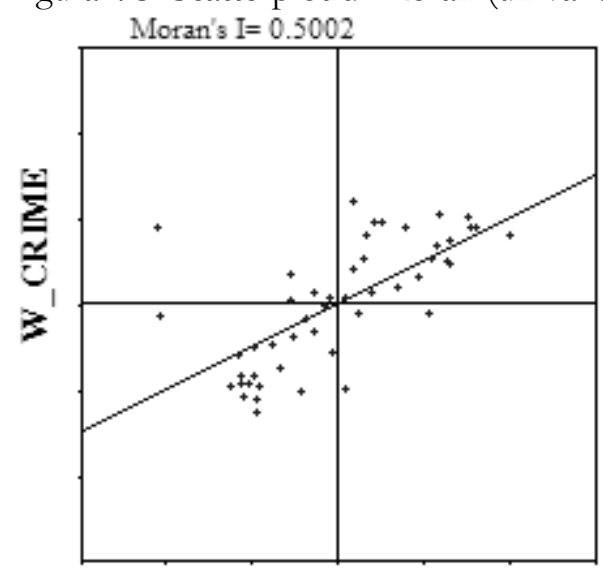

\section{CRIME}

Fonte: Anselin, $2003^{92}$.

Come in un'analisi di correlazione se i punti nello scatterplot di moran presentano un patterns particolare risultano diversi tipi di correlazione spaziale (nulla, positiva e negativa).

\subsubsection{I test locali di dipendenza spaziale}

I test illustrati precedentemente presentano un limite: catturano una relazione di dipendenza spaziale globale. Questi infatti non sono sensibili ad analisi dove esistono clusters o agglomerazioni di valori molto alti in alcuni luoghi e molto bassi in altri. I test non percepiscono differenze in un territorio eterogeneo, dato che forniscono solo un indice globale.

Questo problema è risolto dai test di dipendenza spaziale locale. Infatti, i test locali, $I_{i}$ di Moran, $G_{i}(d)$ Geary e Ord e $G_{i}^{*}(d)$ di Getis e Ord misurano per ogni unità territoriale $i$ la sua relazione spaziale in confronto a tutte le altre unità territoriali.

Gli indici descritti variano come presentati nella tabella sottostante (dove z è il valore della variabile esplicativa nelle unità territoriali $i \operatorname{con} j_{i}$ le unità territoriali vicine). L'interpretazione rimane la stessa, ricordando che questi sono ora calcolati per ogni singola unità territoriale in confronto alle altre, e che quindi risulteranno tanti indici quanti sono i luoghi di riferimento.

${ }^{91}$ Per esempio nel caso che i primi due indici siano non significativi, la significatività del terzo indice potrebbe indicare una concentrazione delle osservazioni solo in alcuni luoghi.

${ }^{92}$ In particolare nel caso si stima la dipendenza spaziale dei crimini nell'esempio Columbus, in ascissa si trova il crimine mentre in ordinata si registra il crimine moltiplicato per la matrice dei contatti. Nell'esempio si identifica, anche la presenza di due outliers. 
Tabella 4.7: Indici locali di dipendenza spaziale;

\begin{tabular}{|c|c|c|}
\hline Test I locale di Moran & Test locale $\mathrm{G}_{\mathrm{i}}(\mathrm{d})$ Geary e Ord & $\begin{array}{c}\text { Test locale } \mathrm{G}_{\mathrm{i}}(\mathrm{d}) \text { di Getis e } \\
\text { Ord }\end{array}$ \\
\hline$I_{i}=\frac{z_{i}}{\sum_{i} z_{i}^{2} / n} \sum_{j \in j i} w_{i j} z_{j}$ & $G_{i}(d)=\frac{\sum_{j=1}^{N} w(d) x_{j}}{\sum_{j=1}^{N} x_{j}} i \neq j$ & $G^{*}{ }_{i}(d)=\frac{\sum_{j=1}^{N} w(d) x_{j}}{\sum_{j=1}^{N} x_{j}}$ \\
\hline
\end{tabular}

Fonte: Anselin, 1998; ${ }^{93}$

A titolo di esempio nella figura 4.4 si presenta lo scattermap e lo scatterplot della distribuzione del crimine in Columbus dove è stato calcolato il test I di Moran locale.

Figura 4.4: ScatterMap e scatterplot della criminalità in Columbus;

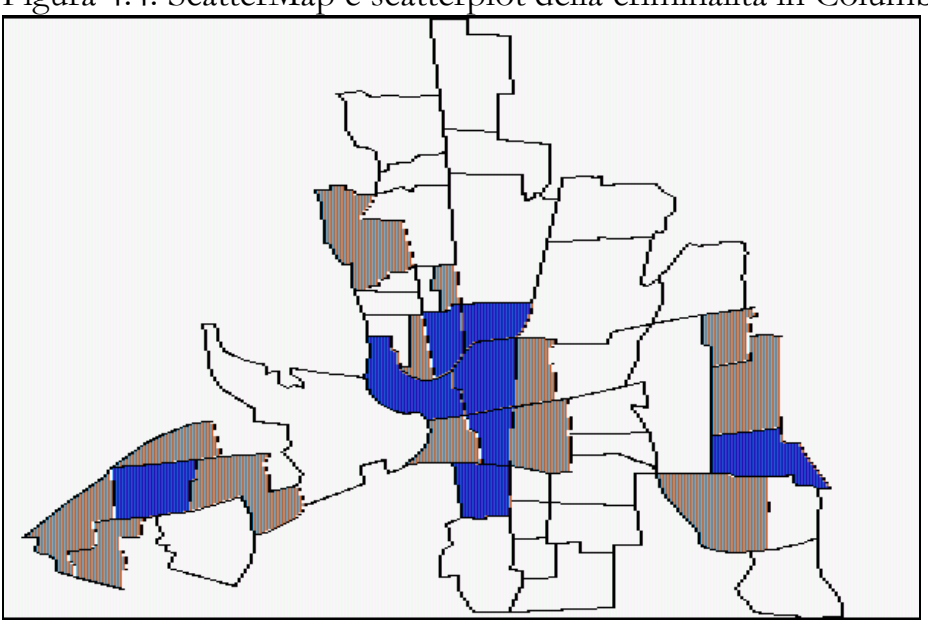

Fonte: Anselin, 2003, p. 76;

La figura 4.4 rileva come, nonostante le zone periferiche siano quelle che registrano un livello di crimine relativo molto basso (si rimanda alla figura 4.1), la criminalità nella periferia si trasmette ai quartieri vicini ed allo stesso modo in quelli centrali. In altre parole nelle zone verdi si registra un alto valore di significatività (più il colore è intenso e più alto è il livello di significatività e correlazione) della diffusione dei crimini nei quartieri.

L'ultimo test locale che abbiamo presentato è lo scattermap. Lo scattermap è un indice visuale di auto-correlazione spaziale che evidenzia la dipendenza spaziale. L'indice mostra in una mappa divisa per le unità territoriali analizzate il valore dello scatterplot per ogni unità territoriale.

Il software applicativo SpaceStat sviluppa tutti i test presentati, mentre per lo scattermap di Moran si deve ricorrere ad una procedura di applicazione in ArcView direttamente fornita con SpaceStat. GeoDa evita questo tipo di problema analizzando direttamente i file shape (mappe) di Arcview e calcolando i test proposti su database georeferenziati ${ }^{94}$.

\footnotetext{
${ }^{93} \mathrm{Nel} \mathrm{G}_{\mathrm{i}}(\mathrm{d})$ di Getis e Ord si permette inoltre che $\mathrm{j}$ sia uguale ad i cioè si valuta anche quando la matrice di contatti w è uguale a 0 . Seguendo questi ragionamenti sono stati calcolati anche altri due indici il New-G ed il New-G* che non presentiamo in questa sede per necessita di sinteticità.

${ }_{94}$ Per un esempio didattico dei vari indici presentati in questo paragrafo e delle mappe, si veda Moreno e Vayà (2000).
} 
La metodologia utilizzata: l'Econometria Spaziale

\subsection{LE ANALISI MULTIVARIATE DI DIPENDENZA SPAZIALE}

L'effetto di dipendenza spaziale, misurato con i vari test presentati nei paragrafi precedenti, può essere presente anche in un modello di regressione. In questo paragrafo cercheremo di proporre un panorama dei fondamentali modelli dell'econometria spaziale che utilizzeremo nella seconda parte di questo lavoro per stimare gli effetti spaziali nei sistemi locali del lavoro. Rimandiamo in ogni modo ai testi di Cliff e Ord (1981) e principalmente al testo di Anselin (1988) per un completo elenco di questo tipo di modelli e delle loro principali caratteristiche.

Due in particolare possono essere le situazioni tipiche in cui è necessario utilizzare un modello spaziale. La prima è quella in cui le variabili del modello sono correlate spazialmente, la seconda è che esista uno schema di dipendenza spaziale negli errori. In entrambi i casi, come già detto, si deve ricorre alla matrice $\mathbf{W}$ dei pesi o contatti che permette di includere l'influenza spaziale delle osservazioni. Per esempio nel caso in cui la variabile sotto studio sia correlata spazialmente, il modello di regressione lineare è il seguente, denominato spatial lag model:

$$
\begin{aligned}
& Y=\rho W \cdot Y+X \beta+u \\
& \mathrm{u} \sim \mathrm{N}\left(0, \sigma^{2} I\right)
\end{aligned}
$$

dove $y$ è un vettore $(\mathrm{Nx} 1), W y$ è il ritardo spaziale della variabile $y, X$ è una matrice di $k$ variabile esogene, $u$ è l'errore, $N$ il numero di osservazioni y e infine, $\rho$ è il parametro che indica la perturbazione. Inoltre si osserva che se erroneamente si omettesse il ritardo spaziale, la dipendenza spaziale si traslerebbe sull'errore, che sembrerebbe quindi correlato spazialmente $^{95}$.

Il secondo modello che presentiamo è quello degli errori denominato spatial error model (o spatial error measurement model) che si presenta nella forma seguente:

$$
\begin{aligned}
& Y=X \beta+u \\
& u=\lambda W u+\varepsilon \\
& \varepsilon \sim \mathrm{N}\left(0, \sigma^{2} I\right)
\end{aligned}
$$

In questo modello denominato di primo ordine ${ }^{96}$, si nota come la matrice dei contatti sia questa volta inserita esclusivamente nell'errore. Il significato dei termini è simile al modello precedente quindi: $y$ è un vettore $(\mathrm{Nx} 1)$, $u$ è l'errore, ritardo spaziale dell'errore, $X$ è una matrice di $k$ variabile esogene, $u$ è l'errore, $N$ il numero di osservazioni y e infine, $\lambda$ è li parametro che indica la perturbazione dell'errore.

Si arriva quindi all'ultimo dei modelli spaziali. Questo modello cattura patterns di dipendenza spaziale sia nell'errore sia nelle variabili esplicative. L'interpretazione dei simboli è simile alla precedente tranne che ci sono due gruppi di variabili e due matrici che

\footnotetext{
95 Moreno e Vayà (2000) in particolare indicano che la correlazione dell'errore potrebbe essere quindi una correlazione spaziale sustantiva e la soluzione sarebbe quella di includere nel modello un ritardo o lag spaziale della variabile correlata spazialmente.

96 Primo ordine perché la matrice degli errori può avere ordini di ritardi superiori. Si capisce intuitivamente che i contatti possono svilupparsi a fasi quindi un set al tempo 1, un altro al tempo 2 e cosi via. Si rimanda ad Anselin (1988) per una specifica più chiara di questi modelli
} 
indicano le rispettive dipendenze. Ulteriormente ai modelli precedenti la matrice $R\left(N^{*} K^{\prime}\right)$ è un vettore di variabili esogene che sono ritardate spazialmente grazie alla matrice $\mathrm{W}_{2}$.

$$
\begin{aligned}
& Y=\rho W_{1} Y+X \beta_{1}+W_{2} R \beta_{2}+u \\
& u=\lambda W_{3} u+\varepsilon \\
& \varepsilon \sim N\left(0, \sigma^{2} I\right)
\end{aligned}
$$

Aggiungiamo inoltre che un errore sulla specificazione della matrice dei contatti ha un'importanza prioritaria sia per la verifica degli errori del modello sia per la stima generale. Moreno e Vayà (2000) descrivono come una erronea definizione dei contatti può avere importante conseguenze sul modello di regressione stimato ${ }^{97}$. L'approccio consigliato è quello di stimare prima una funzione lineare classica, metodo dei minimi quadrati ordinari (OLS o OLS Robust) dopo di che effettuare delle diagnostiche per gli effetti spaziali. Successivamente verificare se uno dei modelli spaziali presentati stimano in maniera più efficace l'evento ${ }^{98}$ tramite modelli di massima verosimiglianza (ML).

Nella tabella 4.8 a conclusione di questo capitolo presentiamo l'elenco dei test sviluppati dal software SpaceStat al fine di effettuare la diagnostica dei modelli di regressione proposti.

L'obiettivo di questo capitolo è stato quello di esporre un panorama dei fondamenti di statistica spaziale prevalentemente operativo e di presentare una descrizione dei modelli che saranno analizzati nella seconda parte di questo scritto e permettere di interpretare $\mathrm{i}$ risultati per chi si avvicina all'econometria spaziale per la prima volta. La scopo della tabella sottostante è il medesimo. I test diagnostici sono molteplici e per una rassegna completa si rimanda ai test di Anselin $(1988,1992)$. Si vuole solo elencare i principali test per verificare la normalità, multicollinearità, eteroschedasticità e autocorrelazione spaziale disponibili nel software messo a disposizione da Anselin e con il quale saranno effettuate le analisi econometriche. In particolare, la tabella sottostante suddivide $i$ test in spaziali e non spaziali. Questi ultimi stimati tramite i minimi quadrati (ordinari e robusti) e i primi (lag e errore) stimati tramite massima verosimiglianza. I test sono elencati in queste categorie principali.

\footnotetext{
${ }^{97}$ Inoltre, i modelli presentati incorporano la dipendenza spaziale tramite modelli autoregressivi di primo ordine detti SAR. Esistono modelli a medie mobile SMA (media mobile spaziale) (Cliff y Ord 1982) e misto SARMA (Anselin e Bera 1998).

98 Tutte le procedure descritte precedentemente sono svolte dal programma di Anselin: SpaceStat (Anselin 1992). In particolare, il Programma SpaceStat, che si basa su uno sviluppo su linguaggio Gauss è stato creato specificatamente per trattare modelli e problematiche legate alla econometria spaziale. Gli indici di Moran e Geary sono entrambi calcolati in tre modi diversi (normale, tramite standardizzazione o permutazione). I risultati possono essere presentati in forma tabellare che include $i$ vari test o tramite un spatial correlogram (dati per costruire uno scattermap con Arcview). Infine i test possono essere calcolati a livello locale o globale, in altre parole per ogni singola unità territoriale o per l'universo preso in considerazione.
} 
La metodologia utilizzata: l'Econometria Spaziale

Tabella 4.8: Analisi con il software SpaceStat: metodi di regressione e test di diagnostica;

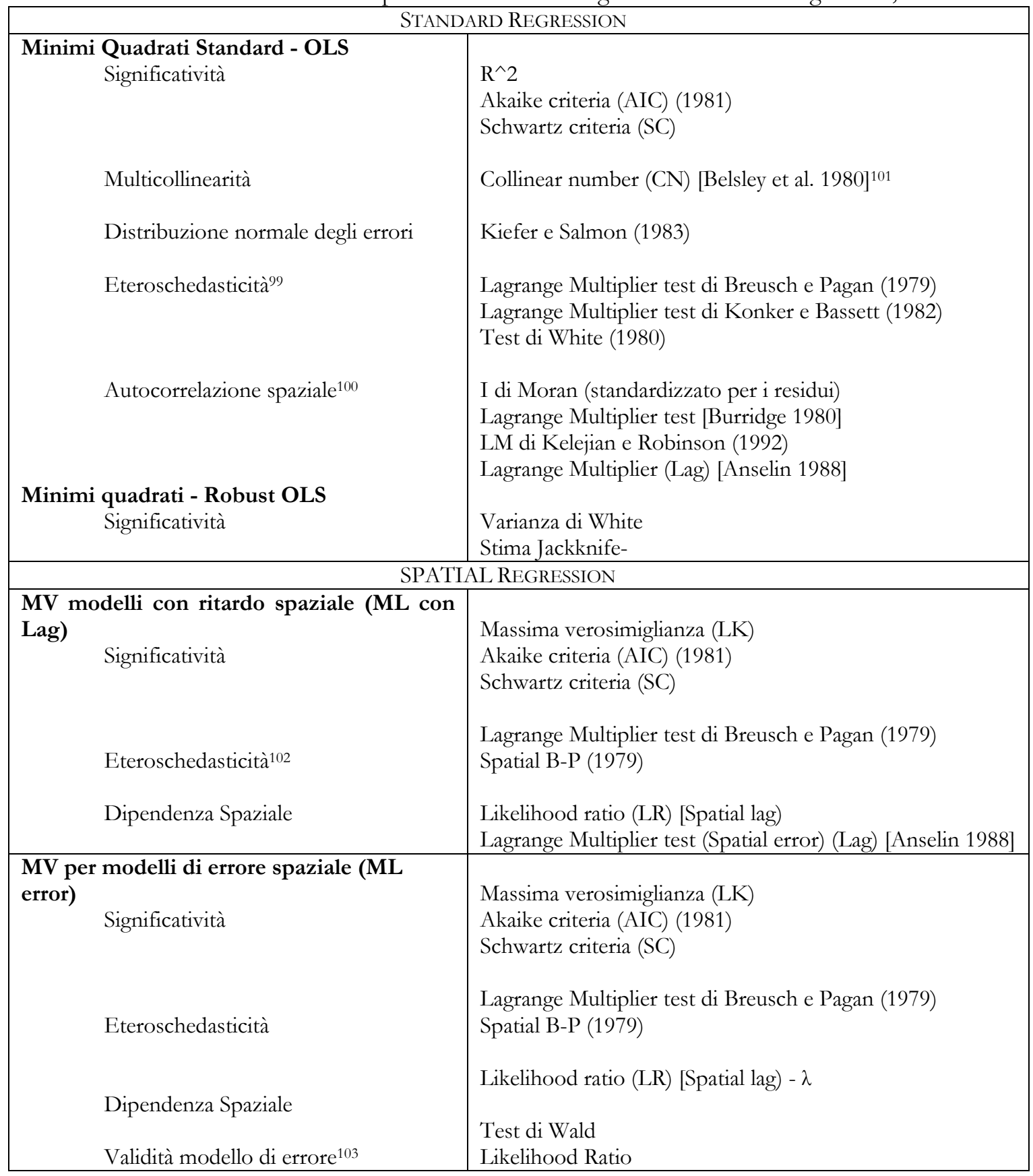

Fonte: nostra elaborazione su Anselin, 1988 e 1992.

\footnotetext{
${ }^{99}$ In caso di eteroschedasticità si ricorda come già detto che la stima del $\mathrm{R}^{\wedge} 2$ può essere sbagliata. Nel caso di assenza di normalità il test fornito è il $\mathrm{KB}$ invece del BP.

100 Per interpretare i risultati dei quattro test si confronta il ritardo con l'errore. Possiamo così rilevare se l'autocorrelazione spaziale si trasmette nella variabile dipendente o nell'errore.

101 Come noto la multicollinearità indica una correlazione tra le variabili inserite nel modello, il collinear number stima questo problema, un numero oltre 20 e 30 pone dei problemi di collinearità. Una stima uguale ad 1 indica una totale mancanza di multicollinearità.

102 LR, Wald e LM (lag) test sono asintoticamente equivalenti, ma i loro valori solitamente rispettano la proporzione $\mathrm{W}>=\mathrm{LR}>=\mathrm{LM}$. (Il Wald si trova con il Lamda ^2)

103 Se questi tests sono altamente significativi indicano che il modello di errore è inappropriato (Anselin, 1992:215)
} 


\section{CAPITOLO V}

\section{ANALISI E MISURAZIONE DELLE FONTI DI COMPETITIVITÀ DEI SLT}

\subsection{LA MISURAZIONE DELLE ECONOMIE ESTERNE E SPATIAL SPILLOVERS}

\subsubsection{Premessa}

Un'ampia letteratura è stata dedicata all'analisi della crescita delle regioni e città attraverso le externalities. Per una rassegna possiamo elencare nell'ultima decade i lavori di Glaeser et al. (1992), Henderson et al. (2003) e Rosenthal e Strange (2002). Altri autori (Deidda et al. 2002) si inseriscono in questo dibattito e sottolineano come gli studi effettuati in questo campo non prendano in considerazione le traformazioni che si stanno sviluppando nelle economie moderne da un'economia basata sulla produzione ad una basata sui servizi dove l'industria turistica sta sempre più svolgendo un ruolo sostanziale ${ }^{104}$.

In questo contesto di riferimento, lo scopo del capitolo è di di misurare le fonti di competitività dei SLT in base alla classificazione proposta nel terzo capitolo (economie esterne e risorse naturali) e utilizzando modelli di Econometria Spaziale tipici dello studio della crescita di regioni e città. Da questo punto di vista considereremo prima le unità territoriali (comuni e SLT) come unità chiuse e poi supereremo i loro confini al fine di analizzare i border effects e gli spillover tra sistemi limitrofi.

La classificazione delle economie esterne che spesso viene utilizzata in questi studi si riferisce principalmente a quella citata nel terzo capitolo quindi a Marshall, Hoover, Jacobs e Romer. Risalendo alla tassonomia proposta, focalizzeremo la nostra attenzione sulle economie di localizzazione (Marshall), di urbanizzazione (Ohlin-Hoover) e di rete (Glaeser et al. 1992).

In riferimento alle economie di rete, lo scopo sarà quello di considerare le unità geografiche non come economie isolate, ma approfondendo le relazioni e i legami intraterritoriali (spillovers spaziali alla Anselin). I contributi più significativi sugli spillovers spaziali sono quelli riferiti ad Anselin ed alle rassegne di Glaeser et al. (1992) e Rosenthal e Strange $(2002)^{105}$. Si ricorda che l'idea principale è che "la prossimità geografica faciliti la trasmissione di idee e la diffusione di conoscenza. [...] attraverso l'imitazione, lo spionaggio, e lo scambio di manodopera tra le imprese, le idee sono velocemente sviluppate e diffuse tra le imprese vicine" (Glaeser et al., 1992:1127). La parola spillovers (spaziali) presuppone, infatti, un certo tipo di "diffusione" della conoscenza a livello spaziale. "...la conoscenza è spesso scambiata senza essere venduta o comprata, la parola spillovers è in questo senso importante" (Rosenthal e Stange, 2002:30).

\footnotetext{
104 Si veda la prima parte sul ruolo del turismo nell'economia mondiale.

105 I principali studi di Gleaser et al. (1992) dimostrano come la competizione e la diversità (diversificazione) promuovono la crescita e l'innovazione, mentre la specializzazione la frena. Risultati diversi sono, invece, proposti da Henderson et al. (1995) che registrano effetti positivi sia sulla diversità che nella specializzazione per industrie di alta tecnologia. Agli stessi risultati perviene De Lucio et al. (2002), le cui analisi riportano un effetto pressoché nullo della diversità e un effetto U-shaped per l'effetto di specializzazione.
} 


\subsubsection{Il framework teorico di analisi delle economie esterne: un modello knowledge-based}

De Lucio et al. (2002) introducono una funzione di impresa Cobb-Douglas ed endogenamente derivano l'indice per misurare le externalities: $Y_{i j t}=A_{i j t} L_{i j t}^{\alpha} K_{i j t}^{\beta}$, dove $Y$ è la produzione, $L$ è il lavoro, $K$ il capitale, $A$ la tecnologia, $i$ è l'industria, $j$ l'unita territoriale, $t$ rappresenta il tempo e $a, \beta$ sono i coefficienti di capitale e lavoro assunti come costanti $\left(K_{i j t} r_{t} / L_{i j t} w_{i j t}=\beta / \alpha\right)^{106}$ come in Grossman e Helpman (1991) e Martin e Ottaviano (1996):

$$
\ln \left(Y_{i j t} / Y_{i j 0}\right)=\beta_{0}+\beta_{1} \ln \left(L_{i j t} / L_{i j 0}\right)+\beta_{2} \ln \left(W_{i j t} / W_{i j 0}\right)+\beta_{3} \ln \left(\phi_{i j t} / \phi_{i j 0}\right)+g(\cdot)
$$

, dove $\phi$ è la produttività ${ }^{107}$.

In altre parole, il tasso di crescita del periodo $Y_{\mathrm{ijt}} / Y_{\mathrm{ij} 0}$ rappresenta la crescita nell'industria $i$ nel periodo considerato ${ }^{108}$. La stima cerca di misurare come questo ultimo varia in base alle variabili esplicative del modello.

Questo modello è temporalmente dinamico, ma non registra il meccanismo di generazione e trasmissione delle esternalità e della conoscenza attraverso le unità territoriali analizzate (economie di rete). Inoltre vogliamo stimare quanto le esternalità e le conoscenze si trasmettano attraverso lo spazio. Dal punto di vista turistico, una destinazione subisce influenze da una destinazione vicina.

$\mathrm{Al}$ fine di includere lo spazio nel modello, dobbiamo considerare che le esternalità dipendono da tre componenti: locale, di network e nazionale /internazionale: $A=A_{\text {local }} \cdot A_{\text {network }} \cdot A_{\text {national } / \text { international }}$. La componente di rete include la conoscenza e le esternalità generate nelle altre città della rete o trasmesse attraverso le unità territoriali analizzate. In altre parole, si cercherà di misurare la crescita di un luogo in base alle economie interne alle imprese, esterne (localizzazione, urbanizzazione), e di rete. Infine, si cercherà di valutare il ruolo di altri determinanti come la diversità ( $a$ la Jacobs).

I network sono considerati endogeni al modello ${ }^{109}$ e l'econometria spaziale (Anselin 1988) fornisce una facile via per risolvere questi problemi, attraverso l'utilizzo di una matrice di contatti nel territorio (spatial contacts $W$ ).

$$
\begin{aligned}
& \text { Dopo la massimizzazione linearizzazione del modello otteniamo: } \\
& \ln \left(Y_{i j t}\right)=\ln \left(A_{i j t}\right)+\alpha \ln \left(L_{i j t}\right)+\beta\left[\ln \left(w_{i j t}\right)+\ln \left(L_{i j t}\right)+\ln (\beta)-\ln (\alpha)-\ln \left(r_{t}\right)\right] \\
& \text { I prezzi risultano endogeni. Il modello è espresso in tassi di crescita. Come in Glaeser et al. (1992), il tasso di } \\
& \text { crescita della tecnologia è assunto come dipendente da un componente locale e da uno globale. }
\end{aligned}
$$

La componete globale $A_{\text {global }}$ cattua modificazione esogene nellat ecnologia. La componente locale $A_{\text {local }}$ is endogenizzata, e come in Grossman e Helpman (1991) e Martin e Ottaviano (1996), il modello considera che la distribuzione delle nuove innovazioni è una funzione lineare e crescente proporzionale al numero passoto di innovazioni locali nell'industria $: d A_{i j t} / d t=A_{i j t}^{*}\left(g_{0}\right)$ [3], dove g è il vettore di variabili esplicative includendo le economie di agglomerazione si deve risolvere l'equazione differenziale con: $A_{j i t}^{\text {local }}=A_{j i t-1}^{\text {local }} e^{g(\cdot) t}$.

107 Se non si hanno sufficenti informazioni disponibili possiamo assumere che sia presente il solo input di lavoro $\Phi=A_{i j t} L_{i j t}^{1-\alpha}$, e il modello è lo stesso a Glaeser at al. (1992) and Henderson et al. (1995). L'equazione può quindi essere stimanta nella forma usuale: $y=X \beta+u$.

108 Questo può essere misurato, per esempio, come la crescita dei lavoratori nell'industria $i$ nel periodo considerato.

${ }^{109} \mathrm{Nel}$ modello di Glaeser et al. (1992) e Henderson et al. (1995) si arriva allo stesso modello partendo dalle ipotesi che le componenti locali territoriali siano esogene. 
Seguendo il modello, le esternalità di rete provengono dalle variabili alle condizioni iniziali negli altri sistemi della rete. Quindi, il modello prende la forma di un cross regressive spatial model: $y=X \beta+W X \gamma+u$. Altre tre opzioni possono essere prese in considerazione e verranno trattate nel prossimo capitolo: la prima è che le esternalità e la conoscenza posso emergere simultaneamente dalla crescita nei settori negli altri luoghi del network (spatial lag model): $y=\rho W y+X \beta+u$; la seconda è che queste due specificazioni possono essere combinate in un regressive-regressive spatial model, includendo i ritardi dei networks della variabile dipendente e di quelle esplicative: $y=\rho W y+X \beta+W X \gamma+u$. Infine, possiamo considerare che $i$ vantaggi competitivi siano trasmessi attraverso shocks stocastici attraverso la rete o semplicemente che gli errori di misurazione siano correlati allo spazio (spatial error model):

$$
\begin{aligned}
& y=X \beta+u \\
& u=\lambda W u+\varepsilon \\
& \varepsilon \sim N\left(0, \sigma^{2} I\right)
\end{aligned}
$$

Tutti questi modelli possono essere combinati al fine di produrre un set di modelli spaziali (Anselin 1988) o estesi per delle più complesse analisi. Questi permettono quindi di stimare simultaneamente le economie di agglomerazione (localizzazione, urbanizzazione, diversità), esternalità di rete e spillovers e di verificare se esistono effetti spaziali tra le unità territoriali esaminate. È naturale che sia possibile che il modello ed i test non registrino effetti spaziali significativi.

\subsection{ANALISI DELLE FONTI DELlA COMPETITIVITÀ NEI SLT. UN PRIMO STUDIO: LE CITTÀ COME UNITÀ DI ANALISI}

\subsubsection{Dati e Variabili}

Dato che i dati disponibili su valore aggiunto e capitale non sono disponibili a livello di impresa o a livello comunale, limitatamente alla filiera proposta, utilizziamo la crescita degli addetti nella filiera dal 1991 al 2001 per stimare le performance dei sistemi locali turistici individuati nel capitolo precedente ${ }^{110}$.

In particolare utilizziamo un modello simile ad una funzione di stima della produzione, con elemento principale la crescita della forza lavoro come in Glaeser et al. (1992), Henderson et al. (1995) e De Lucio et al. (1996). Per le analisi econometriche utilizziamo i dati del censimento ISTAT sull'industria e servizi e popolazione del 1991 e 2001.

In base alle analisi precedenti, ci proponiamo di trovare una forte relazione tra la crescita dell'occupazione turistica e i vantaggi di localizzazione (economie di agglomerazione ed urbanizzazione) degli effetti spaziali spillovers o economie di rete tra $\mathrm{i}$ comuni limitrofi.

I vantaggi di localizzazione possono essere analizzati tramite un approccio sistemico o tramite un'approssimazione alle risorse naturali. In relazione ad un approccio basato sulle risorse naturali, utilizziamo in questo paragrafo delle variabili dummy $(0,1)$ per i diversi sistemi turistici locali evidenziati con la cluster analysis nel capitolo due.

110 Un'altra possibilità sarebbe quella di stimare l'imprenditorialità e stimare il coefficiente di concentrazione per numero di imprese invece che in numero di addetti. 
Al fine di considerare questi aspetti seguiamo l'approccio utilizzato anche in letteratura sulla stima dei modelli di crescita legati a regioni diverse. A titolo di esempio si rimanda alla rassegna di Glaeser et al. (1992) e Rosenthal e Strange (2004) ad un contributo sul turismo di Deidda, Paci ed Usai (2002), ed ad un approccio molto simile in Boix (2004). Costruiamo quindi una serie di indicatori al fine di stimare una funzione che registri effetti legati alle diverse forze competitive. In prima approssimazione, utilizzamo i seguenti indicatori.

- Il coefficiente di localizzazione (LQ), che cattura l'esistenza di spillovers di conoscenza e di manodopera qualificata;

- L'ammontare di addetti nella filiera (Employment);

- L'inverso della dimensione di imprese (Small firm);

- L'inverso dell'indice Hischmann-Herfindahl dentro la filiera, al fine di evidenziare la specializzazione dei fornitori (Filiere) ${ }^{111}$.

- L'esistenza di grandi e medie città d'arte suggerisce che le economie di urbanizzazione possono giocare un ruolo importante per alcuni tipi di turismo (culturale, religioso, etc.). Consideriamo a tal fine l'influenza della popolazione totale del comune (Population) al fine di valutare l'effetto dimensione di Hoover (1937);

- L'inverso dell'indice Hischmann-Herfindahl per tutte le industrie al fine di registrare un effetto diversità (Diversity) come nelle economie alla Jacobs (1969).

- Verifichiamo inoltre l'esistenza di relazioni input-output al di fuori della filiera turistica utilizzando la percentuale dell'occupazione iniziale per settori economici (Agriculture, Manufactures, Construction, Retail, Services).

- Una variabile addizionale è inclusa nel modello ed è costruita come la crescita degli altri settori al di fuori della filiera. Questa variabile è inclusa al fine di comprendere eventuali informazioni omesse riguardanti la crescita degli altri settori (Growth of the other sectors).

Non abbiamo altri dati per verificare un effetto legato alle infrastrutture e riteniamo che essendo correlato al livello di popolazione residente sia incluso nella variabile relativa alla popolazione.

Verifichiamo l'esistenza di spillovers spaziali e di autocorrelazione spaziale impiegando tre tipologie di modelli spaziali (Anselin, 1988, 1992): il modello di ritardo spaziale con variabili esogene (spatial lag - cross regressive mode), il modello di ritardo spaziale (spatial lag) e il modello di errore spaziale (spatial error mode). Anche se abbiamo esaminato nella parte precedente i sistemi locali del lavoro al fine di identificare i sistemi turistici locali, utilizzeremo in questo primo studio i comuni come unità di analisi. Questa proxi ci permetterà di verificare quale è la corretta unità territoriale di riferimento e di misurare eventuali spillovers intra-comunali all'interno di uno stesso sistema locale del lavoro.

Usiamo i comuni all'interno di un sistema locale del lavoro per costruire la matrice dei contatti spaziali (W). A tal fine si assume che all'interno dello stesso sistema locale del lavoro esistono contatti e flussi di informazioni, beni e servizi tra i comuni adiacenti. Infine, verifichiamo la stima dei spatial regimes (Anselin, 1988; 1992) al fine di isolare le performance dei diversi tipi di sistemi turistici identificati con la cluster analysis. Tutte le variabili esplicative (eccetto la variabile crescita negli altri settori - Growth of the other sectors) sono espresse in base all'anno iniziale (1991), al fine di esaminare la relazione di casualità tra le variabili impiegate e la crescita dell'occupazione turistica.

\footnotetext{
$111 \mathrm{Si}$ veda in appendice per l'indice IHHI.
} 
Tabella 5.1: Variabili del modello.

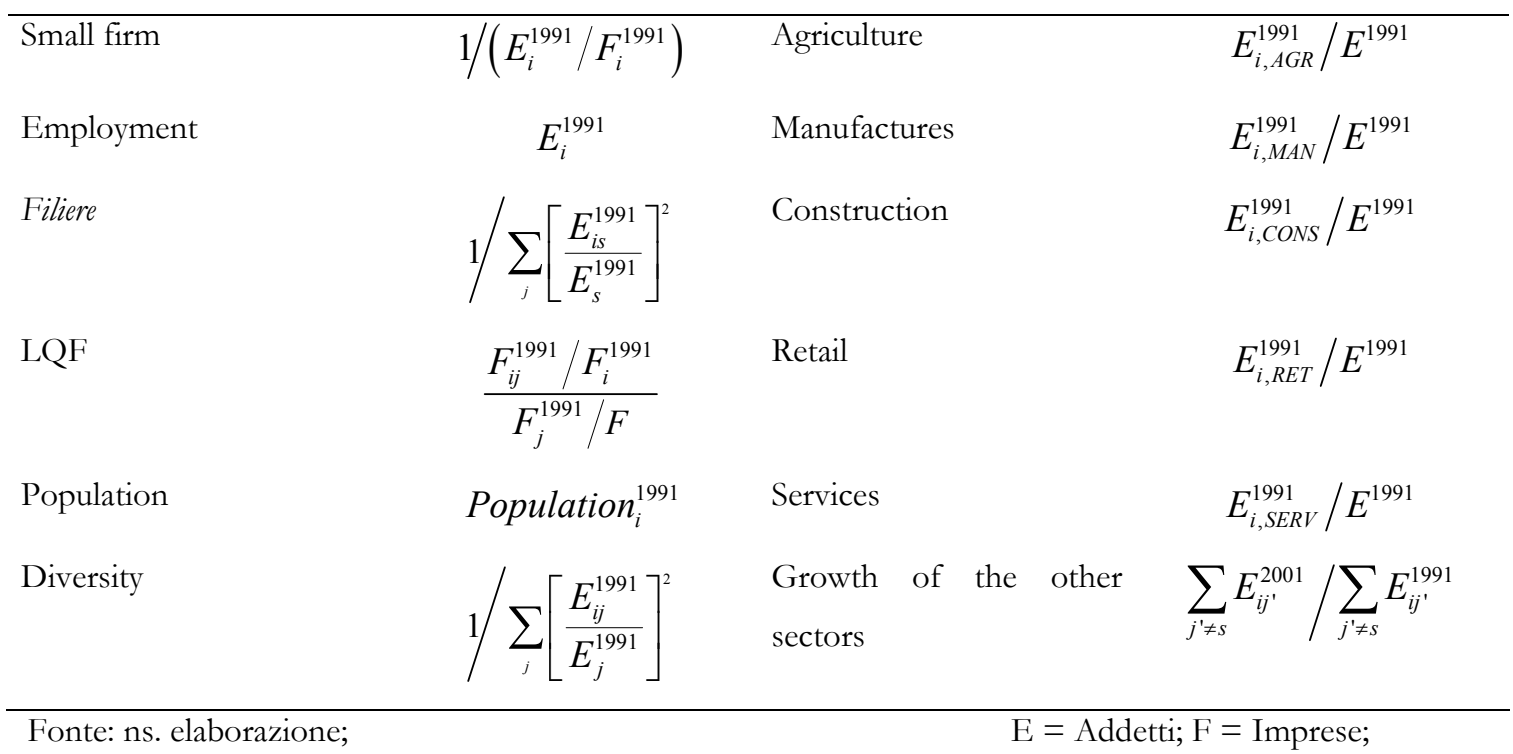

\subsubsection{Modelli non spaziali}

Iniziamo le analisi tramite tre regressioni separate per la localizzazione, l'urbanizzazione e le relazioni input-output. Presentiamo i risultati della stima tramite i minimi quadrati ordinari (OLS) nella tabella 5.2. Gli effetti di localizzazione spiegano oltre il $50 \%$ della varianza mentre gli effetti dell'urbanizzazione input-output ne spiegano un livello intorno al 10\%.

Stimiamo poi il modello di ritardo e il modello di errore che include la localizzazione, l'urbanizzazione e gli effetti input-output tutti allo stesso tempo (tabella 5.3).

Escludiamo le variabili LQF, Education, Agriculture, Building, Retail, Service e le dummy perché non sono statisticamente significative e producono collinearità.

La tabella 5.2 mostra i risultati della prima stima. Il test Koenker-Bassett evidenzia problemi di eteroschedasticità. È calcolato in base ai diversi patterns dei sistemi locali turistici ottenuti con la cluster analysis. Presentiamo i risultati di una stima tramite i minimi quadrati White Robust (OLS - ordinary least squares) (eteroschedasticità robusta) e tramite minimi quadrati generalizzati (FGLS) utilizzando i 5 cluster in modo da modulare la varianza. I risultati sono molto simili e suggeriscono un effetto di eteroschedasticità sul test. Infine, dato che nel modello teorico la variabile dipendente e le variabili esplicative sono espresse in logaritmi $(\mathrm{ln}), \mathrm{i}$ risultati possono essere interpretati come elasticità dirette.

Riguardo alla variabili localizzative il coefficiente più grande è quello relativo alla filiera (inverso dell'indice Hischmann-Herfindahl interno alla filiera turistica) che misura la presenza locale di tutte le componenti della filiera turistica. Inoltre questo indice esprime la coerenza delle attività indicate nella filiera. Questo mostra un coefficiente di $\beta=0.86$. Infatti questa variabile riesce a spiegare fino al $50 \%$ della varianza. La variabile Small firms è positiva e statisticamente significativa con un coefficiente di $\beta=0.08$. Questo indica che l'esistenza di una dimensione di piccola impresa è relazionato ad una migliore performance nella crescita dell'occupazione nella filiera. Il livello iniziale di occupazione (Employment) è negativo con un coefficiente $\beta=-0.11$. Questo risulta comune in questi tipi di modelli ed 
indica che i luoghi con un grande ammontare di addetti nell'industria turistica tendono a crescere meno della media ${ }^{112}$.

Riguardo alle variabili di urbanizzazione, population è statisticamente significative con un coefficiente $\beta=0.094$, mentre diversity (inversa dell'indice $\mathrm{HH}$ calcolato su tutte le industri a livello di due codici ateco) è negativa $\beta=-0.22$. Questo può essere interpretato come una positiva relazione tra la dimensione urbana (infrastrutture e altre amenità) e la diversificazione urbana, che cresce più lentamente nelle città con una più ampia struttura economica diversificata. L'esistenza di una relativa specializzazione nell'industria è positiva e è statisticamente significativa, ma il coefficiente risulta molto piccolo $(\beta=0,02)$.

\subsubsection{Modelli spaziali}

I test spaziali (LM Lag e LM error) suggeriscono l'esistenza di qualche tipo di autocorrelazione spaziale. Dato che il test relativo al modello dell'errore è più grande di quello del ritardo (lag), il modello dell'errore può essere quello più idoneo alla stima (Anselin, 1992) $)^{113}$. In ogni modo il test dell'errore spaziale può essere anche relazionato a problemi di missespecification (McMillen, 2003) del modello.

Iniziamo a stimare un modello che include il ritardo spaziale con variabili esogene (lag - cross regressive spatial model) (tabella 5.3). Dato che tutte le variabili sono in logaritmi e che la matrice dei contatti spaziali è standardizzate per righe, i coefficienti spaziali possono essere interpretati come elasticità dirette della variabile dipendente. In ogni modo ogni coefficiente spaziale esogeno è statisticamente significativo (tabella 5.3, colonna 4) e l'autocorrelazione spaziale continua ad essere principalmente catturata dal test $\mathrm{LM}^{114}$.

I modelli di ritardo spaziale e di errore spaziale sono stimanti tramite la massima verosimiglianza (ML estimation con eteroschedasticità tra gruppi) ed i parametri spaziali sono statisticamente significativi $\varrho=8(\mathrm{lag})$ e $\lambda=0.10$ (errore) $^{115}$. I test Akaike e Schwartz confermano che il modello di errore spaziale è maggiormente idoneo alla stima del modello di ritardo spaziale e del modello non spaziale.

I coefficenti non spaziali sono molto piccoli. Questi suggeriscono due ipotesi: esista una sorte di trasmissione di shokcs stocastici attraverso lo spazio (Anselin, 1988) o che i sistemi locali del lavoro sono la corretta unità di analisi e non la municipalità. L'ultima affermazione converrebbe con le ipotesi di altri studi tra i quali Sforzi (1997a) e Menghinello (2002), che suggeriscono l'utilizzo dei sistemi locali come unità di analisi di tipo socio-economico. Verificheremo questa ipotesi nella seconda parte di questo capitolo.

\footnotetext{
$112 \mathrm{Il}$ coefficente è relazionato al LQ, ma dimostra una migliore performance sul modello. Abbiamo calcolato gli indici di localizzazione utilizzando gli addetti modo da evitare un effetto grande impresa. Abbiamo tentato anche di utilizzare altri coefficenti di specializzazione (Fingleton et al. 2004), ma sono risultati non statisticamente significati.

113 Abbiamo anche effettuato altri tests forniti da SpaceStat 1.91 (Moran's I, KR, Robus LM error and Lag, and SARMA).

114 Dato che il modello mostrava collinearità (condition number $=71.64$ ), si è stimato il modello includendo solo una variabile alla volta. Tuttavia, nessun spatial lag esogeno risulta stato significativo statisticamente.

115 Confermiamo i risultati stimando il modello con i IV (lag, error) and GMM (error).
} 
Analisi delle fonti di competitività dei SLT

Tabella 5.2: Regressioni per gli effetti di localizzazione, urbanizzazione relazioni input-output;

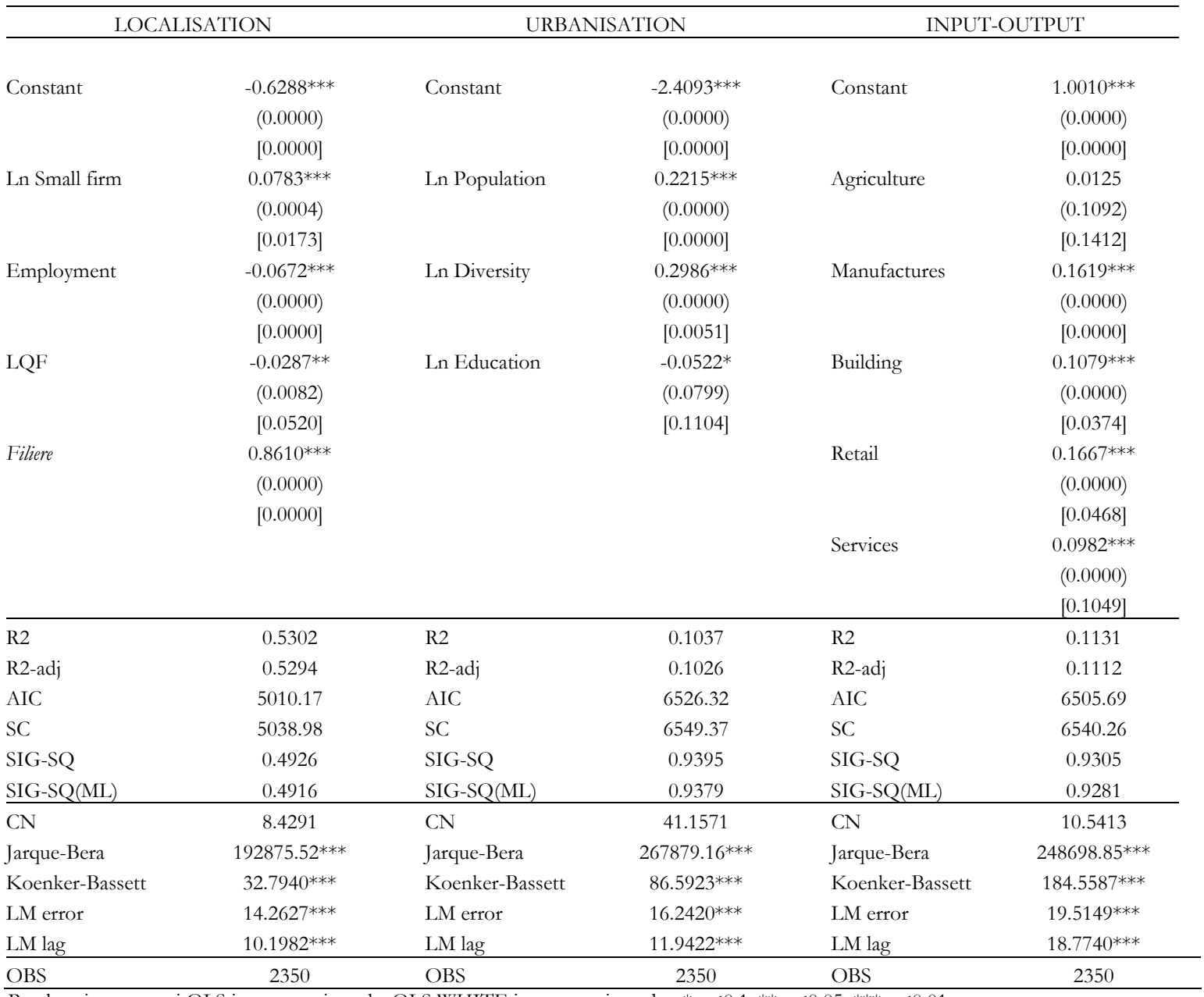

P-values in parentesi OLS in parantesi tonde, OLS WHITE in parentesi quadre. ${ }^{*} \mathrm{p}<0,1 ; * * \mathrm{p}<0,05 ;{ }^{* * *} \mathrm{p}<0,01$. 


\section{Capitolo quinto}

Tabella 5.3: Risultati dei modelli spaziali: unità d'analisi comunale;

\begin{tabular}{|c|c|c|c|c|c|c|}
\hline \multicolumn{7}{|c|}{ Dependent variable: Ln (Labour 2001 / Labour 1991 ) } \\
\hline & OLS & OLS White & FGLS GHET & FGLS GHET & ML LAG GHET & ML ERROR GHET \\
\hline \multirow[t]{2}{*}{ Constant } & $-0.8463 * * *$ & $-0.8463 * * *$ & $-0.8475^{* * *}$ & $-0.6817 * * *$ & $-0.8104 * * *$ & $-0.8548^{* * *}$ \\
\hline & $(0.0000)$ & $(0.0000)$ & $(0.0000)$ & $(0.0001)$ & $(0.0000)$ & $(0.0000)$ \\
\hline \multirow[t]{2}{*}{ Ln Small firm 1991} & $0.0815^{* * *}$ & $0.0815^{* *}$ & $0.0760^{* * *}$ & $0.0790 * * *$ & $0.0730^{* * *}$ & $0.0768 * * *$ \\
\hline & $(0.0001)$ & $(0.0055)$ & $(0.0004)$ & $(0.0003)$ & $(0.0007)$ & $(0.0004)$ \\
\hline \multirow[t]{2}{*}{ Ln Employment 1991} & $-0.1164^{* * *}$ & $-0.1164 * * *$ & $-0.1113 * * *$ & $-0.1121 * * *$ & $-0.1114 * * *$ & $-0.1094 * * *$ \\
\hline & $(0.0000)$ & $(0.0000)$ & $(0.0000)$ & $(0.0000)$ & $(0.0000)$ & $(0.0000)$ \\
\hline \multirow[t]{2}{*}{ Ln Filiere1991 } & $0.9078 * * *$ & $0.9078^{* * * *}$ & $0.8952^{* * *}$ & $0.8962^{* * * *}$ & $0.8915^{* * *}$ & $0.8921 * * *$ \\
\hline & $(0.0000)$ & $(0.0000)$ & $(0.0000)$ & $(0.0000)$ & $(0.0000)$ & $(0.0000)$ \\
\hline \multirow[t]{2}{*}{ Ln Population 1991} & $0.0944 * * *$ & $0.0944 * * *$ & $0.0934 * * *$ & $0.0963^{* * *}$ & $0.0890 * * *$ & $0.0933^{* * *}$ \\
\hline & $(0.0000)$ & $(0.0000)$ & $(0.0000)$ & $(0.0000)$ & $(0.0000)$ & $(0.0000)$ \\
\hline \multirow[t]{2}{*}{ Ln Diversity1991 } & $-0.2212^{* * *}$ & $-0.2212^{* * *}$ & $-0.2279 * * *$ & $-0.2200 * * *$ & $-0.2252 * * *$ & $-0.2262^{* * *}$ \\
\hline & $(0.0000)$ & $(0.0059)$ & $(0.0000)$ & $(0.0000)$ & $(0.0000)$ & $(0.0000)$ \\
\hline \multirow[t]{2}{*}{ Ln Manufactures 1991} & $0.0304 * *$ & $0.0304 *$ & $0.0241 * * *$ & $0.0201 *$ & $0.0234 * *$ & $0.0225^{* *}$ \\
\hline & $(0.0037)$ & $(0.0697)$ & $(0.0167)$ & $(0.0564)$ & $(0.0199)$ & $(0.0266)$ \\
\hline \multirow[t]{2}{*}{ Ln Growth other sectors } & $0.1645^{* * *}$ & $0.1645^{* * *}$ & $0.1501 * * *$ & $0.1495^{* * *}$ & $0.1483^{* * *}$ & $0.1484 * * *$ \\
\hline & $(0.0000)$ & $(0.0000)$ & $(0.0000)$ & $(0.0000)$ & $(0.0000)$ & $(0.0000)$ \\
\hline \multirow[t]{2}{*}{$W^{*}$ Ln Small firm 1991} & & & & -0.0393 & & \\
\hline & & & & $(0.4674)$ & & \\
\hline \multirow[t]{2}{*}{ W * Ln Labour1991 } & & & & -0.0187 & & \\
\hline & & & & $(0.5410)$ & & \\
\hline \multirow[t]{2}{*}{ W * Ln Filiere 1991} & & & & 0.0465 & & \\
\hline & & & & $(0.4848)$ & & \\
\hline \multirow[t]{2}{*}{$W^{*}$ Ln Population 1991} & & & & -0.0097 & & \\
\hline & & & & $(0.7578)$ & & \\
\hline \multirow[t]{2}{*}{ W* Ln Diversity 1991} & & & & -0.0484 & & \\
\hline & & & & $(0.5455)$ & & \\
\hline \multirow[t]{2}{*}{ W * Ln Manufactures 1991} & & & & 0.0309 & & \\
\hline & & & & $(0.1861)$ & & \\
\hline \multirow[t]{2}{*}{ W * Ln Growth other sectors } & & & & 0.0405 & & \\
\hline & & & & $(0.5966)$ & & \\
\hline \multirow[t]{2}{*}{$\varrho$} & & & & & 0.0815 & \\
\hline & & & & & $(0.0045)$ & \\
\hline \multirow[t]{2}{*}{$\lambda$} & & & & & & 0.1098 \\
\hline & & & & & & $(0.0017)$ \\
\hline R2 & 0.5471 & 0.5471 & 0.5312 & 0.5325 & 0.5307 & 0.5293 \\
\hline R2-adj & 0.5457 & 0.5457 & & & & \\
\hline SQ Corr & & & 0.5469 & 0.5476 & 0.5486 & 0.5469 \\
\hline AIC & 4930.38 & 4930.38 & & & 4881.65 & 4873.3 \\
\hline SC & 4976.48 & 4976.48 & & & 4933.51 & 4919.4 \\
\hline $\mathrm{CN}$ & 29.27 & & & 71.64 & & \\
\hline Jarque-Bera & $204183^{* * *}$ & & & & & \\
\hline Koenker-Bassett & $34.52^{* * *}$ & & & & & \\
\hline WALD HET & & & $76.80^{* * * *}$ & $73.7603^{* * *}$ & & \\
\hline LR HET & & & & & $44.5435^{* * *}$ & $48.0926^{* * *}$ \\
\hline LM error & $8.9121 * * *$ & & $8.7649 * * *$ & $8.7923^{* * *}$ & & \\
\hline LM lag & $5.9290 * * *$ & & $6.7948^{* * *}$ & $8.4803^{* * *}$ & & \\
\hline OBS & 2350 & 2350 & 2350 & 2350 & 2350 & 2350 \\
\hline
\end{tabular}

P-values in parentesi. ${ }^{*} \mathrm{p}<0,1 ;{ }^{* *} \mathrm{p}<0,05 ;{ }^{* * *} \mathrm{p}<0,01$. 


\subsubsection{Regimi Spaziali}

Infine verifichiamo la performance dei modelli sulle diverse tipologie di sistemi locali turistici utilizzando i regimi spaziali. Questo modello è descritto in Anselin (1988 e 1992). Questo procedimento utilizza una variabile come indicatore in modo da separare le pendenze delle differenti intercette e dei coefficienti per i vari sistemi locali turistici:

$$
\left[\begin{array}{l}
y_{1} \\
y_{2} \\
y_{3} \\
y_{4} \\
y_{5}
\end{array}\right]=\left[\begin{array}{ccccc}
X_{1} & 0 & 0 & 0 & 0 \\
0 & X_{2} & 0 & 0 & 0 \\
0 & 0 & X_{3} & 0 & 0 \\
0 & 0 & 0 & X_{4} & 0 \\
0 & 0 & 0 & 0 & X_{5}
\end{array}\right]\left[\begin{array}{l}
\beta_{1} \\
\beta_{2} \\
\beta_{3} \\
\beta_{4} \\
\beta_{5}
\end{array}\right]+\left[\begin{array}{l}
u_{1} \\
u_{2} \\
u_{3} \\
u_{4} \\
u_{5}
\end{array}\right]
$$

Utilizzando questa struttura possiamo stimare il modello OLS e il modello degli errori spaziali nella forma usuale: $y^{*}=X^{*} \beta^{*}+u^{*}$.

I risultati, in tabella 5.4, mostrano alcune interessanti informazioni. Il modello ha una buona performance sul cluster \#2 (città d'arte) e \#4 (sistemi turistici). Gli altri coefficienti negli altri tre clusters non sono statisticamente significativi (distretti turistici). Il Test di Chow-Wald $\left(\chi^{2}\right)$ conferma inoltre che esistono dei problemi di instabilità strutturale nel modello considerato principalmente associato alle variabili dei fornitori della filiera. 


\section{Capitolo quinto}

Tabella 5.4: Regimi spaziali;

\begin{tabular}{|c|c|c|c|c|c|c|c|c|c|c|}
\hline & \multicolumn{5}{|c|}{ OLS } & \multicolumn{5}{|c|}{ ML ERROR } \\
\hline & $\mathrm{C} 1$ & $\mathrm{C} 2$ & C3 & $\mathrm{C} 4$ & C5 & $\mathrm{C} 1$ & $\mathrm{C} 2$ & $\mathrm{C} 3$ & $\mathrm{C} 4$ & C5 \\
\hline \multirow[t]{2}{*}{ Constant } & -0.7383 & $-0.8762^{* * *}$ & -0.3603 & $-1.1228 * *$ & -3.3009 & -0.6785 & $-0.8747 * * *$ & -0.4695 & $-1.2254 * *$ & -3.3009 \\
\hline & $(0.1307)$ & $(0.0000)$ & $(0.6652)$ & $(0.0036)$ & $(0.5073)$ & $(0.1753)$ & $(0.0000)$ & $(0.5865)$ & $(0.0018)$ & $(0.5012)$ \\
\hline \multirow[t]{2}{*}{ Ln Small firm 1991} & -0.1202 & $0.1094 * * *$ & -0.0462 & -0.0137 & 1.0647 & -0.1115 & $0.1102^{* * *}$ & -0.0592 & -0.0159 & 1.0647 \\
\hline & $(0.4069)$ & $(0.0000)$ & $(0.7590)$ & $(0.8030)$ & $(0.6401)$ & $(0.4427)$ & $(0.0000)$ & $(0.6985)$ & $(0.7708)$ & $(0.6347)$ \\
\hline \multirow[t]{2}{*}{ Ln Employment 1991} & -0.1075 & $-0.1262^{* * *}$ & -0.1190 & $-0.1840^{* * *}$ & 1.2933 & -0.0947 & $-0.1225^{* * *}$ & -0.1384 & $-0.1955^{* * *}$ & 1.2933 \\
\hline & $(0.1022)$ & $(0.0000)$ & $(0.1482)$ & $(0.0001)$ & $(0.4734)$ & $(0.1601)$ & $(0.0000)$ & $(0.1039)$ & $(0.0000)$ & $(0.4730)$ \\
\hline \multirow[t]{2}{*}{ Ln Filiere ${ }_{1991}$} & 0.0653 & $0.9237^{* * *} *$ & -0.0334 & $0.9701 * * *$ & -0.2625 & 0.0514 & $0.9223^{* * *}$ & -0.0503 & $0.9718^{* * *}$ & -0.2625 \\
\hline & $(0.7207)$ & $(0.0000)$ & $(0.9099)$ & $(0.0000)$ & $(0.9071)$ & $(0.7750)$ & $(0.0000)$ & $(0.8653)$ & $(0.0000)$ & $(0.8971)$ \\
\hline \multirow[t]{2}{*}{ Ln Population 1991} & $0.1261 *$ & $0.0998^{* * *}$ & 0.1501 & $0.1605^{* *}$ & -0.5484 & 0.1187 & $0.0967 * * *$ & 0.1741 & $0.1720^{* *}$ & -0.5484 \\
\hline & $(0.0973)$ & $(0.0000)$ & $(0.2151)$ & $(0.0049)$ & $(0.6722)$ & $(0.1357)$ & $(0.0000)$ & $(0.1658)$ & $(0.0031)$ & $(0.6900)$ \\
\hline \multirow[t]{2}{*}{ Ln Diversity 1991} & 0.0501 & $-0.2052^{* * *}$ & -0.1569 & -0.2091 & 1.9407 & 0.0242 & $-0.1980^{* * *}$ & -0.1503 & -0.1801 & 1.9407 \\
\hline & $(0.7977)$ & $(0.0000)$ & $(0.6374)$ & $(0.1690)$ & $(0.5882)$ & $(0.9028)$ & $(0.0000)$ & $(0.6507)$ & $(0.2377)$ & $(0.5965)$ \\
\hline \multirow[t]{2}{*}{ Ln Manufactures 1991} & 0.0000 & $0.0338^{* * *}$ & -0.0003 & $0.0933 * * *$ & 0.2524 & 0.0009 & $0.0334^{* *}$ & -0.0068 & $0.0852^{*}$ & 0.2524 \\
\hline & $(0.9992)$ & $(0.0051)$ & $(0.9956)$ & $(0.0052)$ & $(0.6213)$ & $(0.9788)$ & $(0.0054)$ & $(0.9079)$ & $(0.0121)$ & $(0.6428)$ \\
\hline \multirow[t]{2}{*}{$\begin{array}{l}\text { Ln Growth } \\
\text { other sectors }\end{array}$} & 0.0017 & $0.1963^{* * *}$ & -0.1276 & $0.1630^{* *}$ & 0.9506 & 0.0096 & $0.1927^{* * *}$ & -0.1401 & $0.1584^{*}$ & 0.9506 \\
\hline & $(0.9859)$ & $(0.0000)$ & $(0.5115)$ & $(0.0422)$ & $(0.8196)$ & $(0.9194)$ & $(0.0000)$ & $(0.4696)$ & $(0.0463)$ & $(0.8201)$ \\
\hline \multirow[t]{2}{*}{$\lambda$} & & & & & & $0.1124 * *$ & & & & \\
\hline & & & & & & $(0.0013)$ & & & & \\
\hline R2 & 0.5616 & & & & & 0.5613 & & & & \\
\hline R2-adj & 0.5542 & & & & & & & & & \\
\hline SQ Corr & & & & & & 0.5614 & & & & \\
\hline $\mathrm{AIC}$ & 4917.99 & & & & & 4909.60 & & & & \\
\hline SC & 5148.48 & & & & & 5140.08 & & & & \\
\hline SIG-SQ & 0.466737 & & & & & 0.4563 & & & & \\
\hline SIG-SQ(ML) & 0.458793 & & & & & & & & & \\
\hline $\mathrm{CN}$ & 171.87 & & & & & & & & & \\
\hline Jarque-Bera & $225560 * * *$ & & & & & & & & & \\
\hline Chow - Wald & $2.3851^{* * *}$ & & & & & $77.0352 * * *$ & & & & \\
\hline Koenker-Bassett & 2.6279 & & & & & & & & & \\
\hline Breusch-Pagan & & & & & & $65.3377^{* * * *}$ & & & & \\
\hline Spatial BP & & & & & & $65.3528 * * *$ & & & & \\
\hline LM ERROR & $7.7258^{* * *}$ & & & & & & & & & \\
\hline LM LAG & $5.2390 * * *$ & & & & & & & & & \\
\hline OBS & 193 & 1708 & 86 & 355 & 8 & 193 & 1708 & 86 & 355 & 8 \\
\hline
\end{tabular}




\subsubsection{Conclusioni del primo studio}

L'obiettivo di questo capitolo è stato di analizzare le fonti della competitività dei sistemi turistici locali identificati nel capitolo precedente. In particolare lo scopo è stato di misurare le economie esterne per i comuni: sia a livello di singolo comune che a livello di rete. In relazione a questo argomento l'ipotesi principale è stata quella di differenziare le fonti principali di rendimenti crescenti di scala nel processo produttivo in economie esterne. Utilizzando il framework presentato nel terzo capitolo di questa tesi ed utilizzando le economie di localizzazione marshalliane, le economie di urbanizzazione à la Hoover (1937) e di rete tramite gli spillovers spaziali ${ }^{116}$.

Nella parte precedente abbiamo individuato i sistemi turistici locali italiani, in questo primo studio abbiamo verificato l'applicabilità di modelli econometrici spaziali relativamente ai diversi tipi di sistemi turistici locali identificati tramite la cluster analysis. I test spaziali (LM Lag e LM error) suggeriscono l'esistenza di qualche tipo di autocorrelazione spaziale. I parametri spaziali sono significativi (con un $\varrho=0.8$ del lag e $\lambda=0.10$ dell'errore) e quindi si presume che esista un network a livello della filiera all'interno dei singoli sistemi locali del lavoro. Da questo punto di vista questi ultimi e non il comune si presumono come la corretta unità di analisi come nell'ipotesi di Sforzi (1997a) e Menghinello (2002).

I modelli dei regimi spaziali hanno una ottima performance sui cluster Città d'arte e Sistemi turistici specializzati (in una sola parte della filiera). Al contrario i coefficienti del cluster dei presunti distretti turistici non sono statisticamente significativi. Il modello proviene principalmente da studi provenienti dall'Economia Urbana e risulta totalmente soddisfacente per medie e grandi città, ma non riesce a spiegare le performance e la crescita dei distretti turistici e delle città più piccole.

In sintesi, i tassi di crescita più alti sono associati alla presenza locale di tutte le fasi della filiera turistica. Infatti questa variabile riesce a spiegare circa il $50 \%$ della varianza e conferma che la filiera proposta è idonea allo studio del fenomeno in questione. Inoltre l'esistenza di piccole imprese è relazionata a migliori performance nella crescita dell'occupazione turistica nel municipio. Importanti implicazioni emergono da tali risultati per i policy makers e suggeriscono un maggiore attenzione nel promuovere lo sviluppo di ogni anello della filiera turistica.

In relazione agli ulteriori sviluppi della ricerca, nel prossimo paragrafo applicheremo questa metodologia ai sistemi locali del lavoro e alle reti di sistemi locali.

\subsection{ANALISI DELLE FONTI DELLA COMPETITIVITÀ NEI SLT. UN SECONDO STUDIO: I SISTEMI LOCALI COME UNITÀ DI ANALISI}

\subsubsection{Dati e Variabili}

In questa seconda analisi effettueremo nuovamente le elaborazioni utilizzando come unità di analisi i sistemi locali sia verificando la presenza di economie esterne che di spillovers nei sistemi limitrofi. In base alle analisi precedenti ci proponiamo di trovare una forte correlazione tra la crescita dell'occupazione turistica e $\mathrm{i}$ vantaggi di localizzazione (economie di agglomerazione ed urbanizzazione).

116 Si rimanda per questo punto necessariamente al capitolo due di questo scritto. 
I vantaggi di localizzazione possono essere analizzati tramite un approccio sistemico o tramite un'approssimazione alle risorse naturali. In relazione ad un approccio basato sulle risorse naturali nel paragrafo precedente abbiamo utilizzato delle variabili dummy $(0,1)$ per i diversi sistemi turistici locali evidenziati con la cluster analysis.

In questo paragrafo inseriamo, oltre alle variabili presentate, una variabile sperimentale dummy PACA (Patrimonio artistico, culturale ed ambientale) (Lazzeretti, 1997, 2001), che utilizza le risorse naturali (natural endowments) dei sistemi locali turistici. Tale variabile è costruita in base all'integrazione di molteplici fonti di dati. In prima istanza, abbiamo cercato di valutare la presenza di dotazioni del fattore PACA nel territorio, consci del fatto che il nostro approccio è soprattutto un primo tentativo di approssimazione. Abbiamo utilizzato:

- come proxi del patrimonio artistico e culturale l'elenco dei comuni dell'Unesco considerati patrimonio dell'umanità;

- come patrimonio naturale si sono utilizzati invece tre indicatori integrati tra loro: la presenza di parchi nazionali nel territorio; le bandiere blu (blue flags) per la qualità delle spiagge; ed infine, la presenza di destinazioni sciistiche registrate dal Touring club.

Le risorse presenti a livello comunale sono state poi aggregate per sistema locale ${ }^{117}$. Presentiamo la mappa della dummy $P A C A$ nella figura numero 5.1. Si registra una distribuzione territoriale simile a quella dei sistemi locali turistici. Si presume, quindi, una certa dipendenza della competitività di questi sistemi in base alla dotazione del fattore $P A C A$.

Costruiamo quindi una serie di indicatori, al fine di stimare una funzione che registri effetti legati alle diverse forze competitive (economie esterne) come già presentata nell'analisi del paragrafo precedente.

Figura 5.1: Il $P A C A$ nei SLL in Italia;

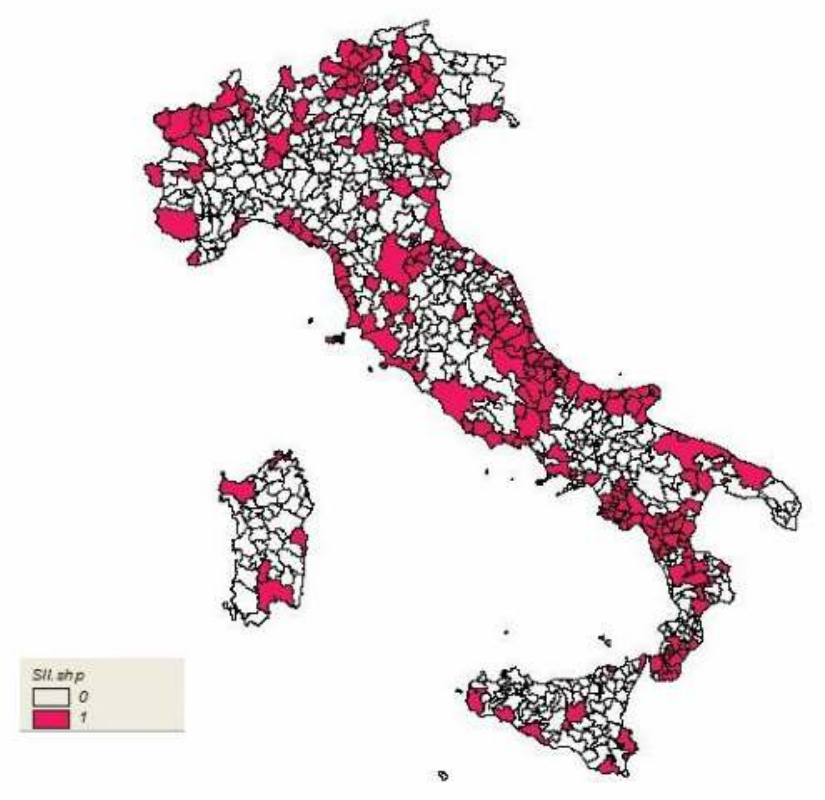

Fonte: nostra elaborazione su fonti UNESCO, Touring Club , Blue Flags e FederPArchi;

${ }^{117}$ L'elenco di queste risorse sono elencate in appendice. 
Cercheremo poi di analizzare quali sono le motivazioni per la crescita di alcuni sistemi locali invece di altri. Rappresentiamo i sistemi locali che registrano delle migliori performance nella figura 5.2, già presentata nel secondo capitolo. Si nota che i patterns territoriali registrano un autonoma distribuzione e non seguono nessuna delle mappe presentate nel capitolo due relative ai SLT.

Figura 5.2: La crescita dell'occupazione della filiera turistica nei SLL, 1991-2001;

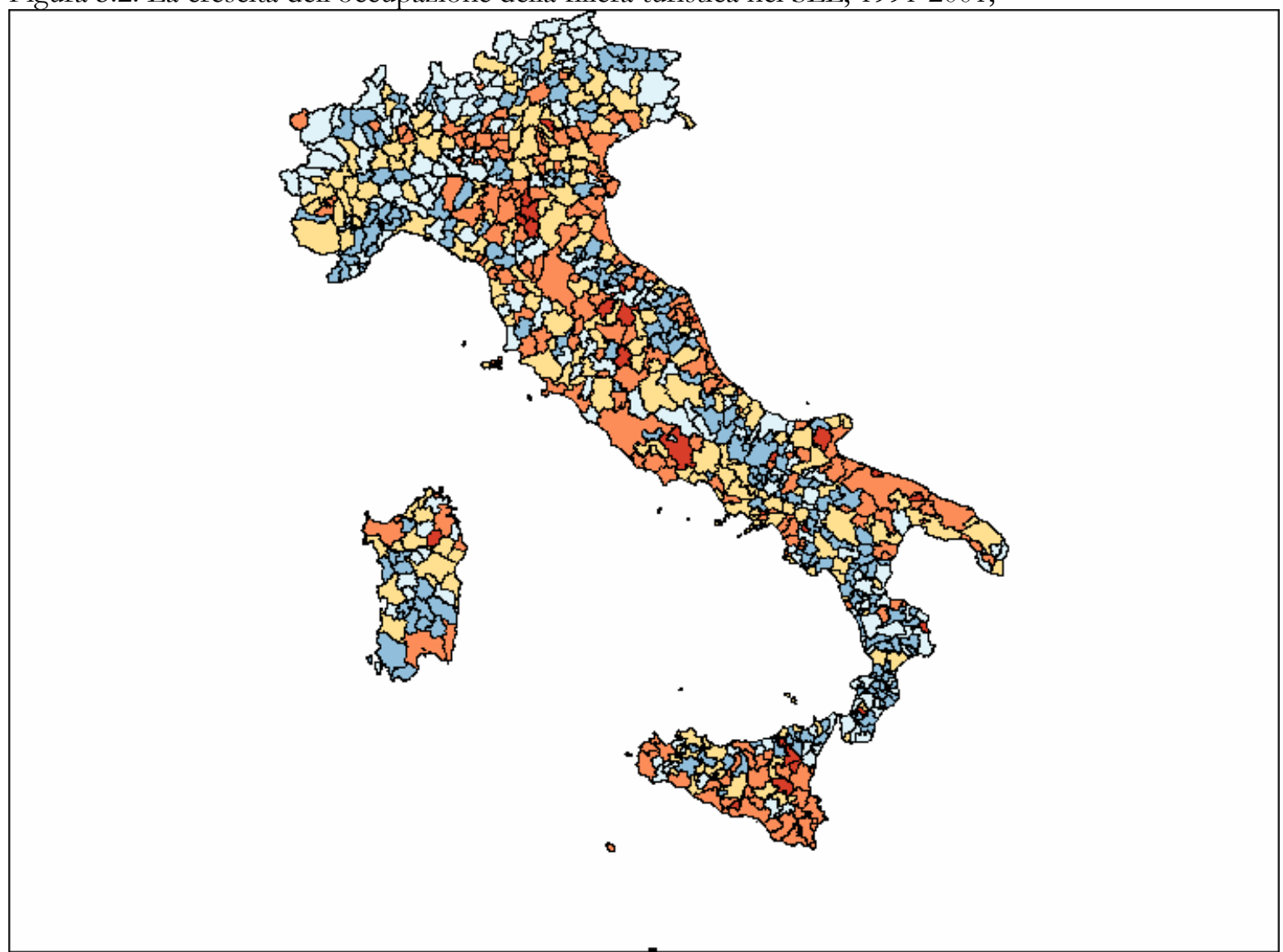

Fonte: nostra elaborazioni su dati ISTAT 1991-2001;

Verifichiamo l'esistenza di spillovers spaziali e di autocorrelazione spaziale utilizzando tre tipologie di modelli spaziali (Anselin, 1988, 1992): il modello di ritardo spaziale con variabili esogene (spatial lag - cross regressive model), il modello di ritardo spaziale (spatial lag) ed il modello di errore spaziale (spatial error model).

In questa seconda elaborazione utilizzeremo come unità di analisi i sistemi locali, a differenza dell'analisi precedente effettuata a livello municipale. Questa proxi ci permetterà anche di verificare quale è la corretta unità territoriale di riferimento e di misurare eventuali spillovers tra sistemi locali limitrofi. Costruiamo la matrice dei contatti spaziali (W), assumendo che i sistemi locali del lavoro adiacenti condividano un certo grado di contatti e dei flussi di informazioni, beni e servizi.

La matrice per ogni sistema locale individua i sistemi locali adiacenti e crea una rete di sistemi locali in base al contatto (adiacenza). Infine utilizziamo la stima dei regimi spaziali tramite l'applicazione di SpaceStat al fine di isolare le performance dei diversi tipi di sistemi turistici identificati con la cluster analysis, come nel paragrafo precedente.

Tutte le variabili esplicative sono espresse in base all'anno iniziale (1991), al fine di analizzare la relazione di casualità tra le variabili utilizzate e la crescita dell'occupazione 
turistica tranne la variabile $P A C A^{118}$ che però risulta una variabile dummy non dinamica temporalmente.

Tabella 5.5: Variabili del modello.

\begin{tabular}{|c|c|c|c|}
\hline Small firm & $1 /\left(E_{i}^{1991} / F_{i}^{1991}\right)$ & Agriculture & $E_{i, A G R}^{1991} / E^{1991}$ \\
\hline Employment & $E_{i}^{1991}$ & Manufactures & $E_{i, M A N}^{1991} / E^{1991}$ \\
\hline Filiere & $1 / \sum_{j}\left[\frac{E_{i s}^{1991}}{E_{s}^{1991}}\right]^{2}$ & Construction & $E_{i, \text { CONS }}^{1991} / E^{1991}$ \\
\hline LQF & $\frac{F_{i j}^{1991} / F_{i}^{1991}}{F_{j}^{1991} / F}$ & Retail & $E_{i, R E T}^{1991} / E^{1991}$ \\
\hline Population & Population $_{i}^{1991}$ & Services & $E_{i, S E R V}^{1991} / E^{1991}$ \\
\hline Diversity & $1 / \sum_{j}\left[\frac{E_{i j}^{1991}}{E_{j}^{1991}}\right]^{2}$ & PACA dummy & $1 ; 0$ \\
\hline
\end{tabular}

$\mathrm{E}=$ Addetti; F = Imprese;

\subsubsection{Modelli non spaziali}

Iniziamo le analisi tramite le tre regressioni separate come nella sezione precedente: la localizzazione, l'urbanizzazione e le relazioni input-output,. Gli effetti di localizzazione spiegano oltre il $70 \%$ della varianza mentre gli effetti di urbanizzazione ed input-output spiegano una varianza intorno al $12 \%$ in tabella 5.6. Inseriamo inoltre la variabile dummy $P A C A$ che risulta significativa e positiva $(0,13)$ come ci si aspettava. Successivamente si stima un modello che includa la localizzazione, l'urbanizzazione e gli effetti input-output. Inseriamo in questo modello anche delle dummy per i diversi tipi di sistemi locali turistici identificati. Escludiamo le variabili LQF, Education, Agriculture, Building, Retail, Service e le dummy perché non sono statisticamente significative e producono collinearità.

La tabella 5.7 evidenzia i risultati della stima ${ }^{119}$. Questi sono molto simili e suggeriscono un bias di eteroschedasticità sul test. Infine dato che nel modello teorico la variabile dipendente e le variabili esplicative sono espresse in logaritmi (ln), i risultati possono essere interpretati come elasticità dirette.

Riguardo alle variabili localizzative, il coefficiente più grande nella stima precedente era quello relativo alla filiera (inverso dell'indice Hischmann-Herfindahl interno alla filiera turistica) che misura la presenza locale di tutte le parti della filiera turistica. In questa sezione il coefficiente è ridimensionato e sembra che la presenza della filiera allargata a livello del sistema locale sia meno importante che a livello municipale. La variabile small firm

\footnotetext{
118 Omettiamo la variabile crescita negli altri settori (Growth of the other sectors) per problemi di causalità dato che non è stimata nel anno 1991.

${ }^{119}$ Il test Koenker-Bassett evidenzia problemi di eteroschedasticità. È calcolato in base ai diversi patterns dei sistemi locali turistici ottenuti con la cluster analysis.
} 
è negativa, ma risulta non statisticamente significativa ${ }^{120}$. Il livello iniziale di occupazione è negativo con un coefficiente $\beta=-0.50$. Questo risulta comune in questo tipi di modelli, come nella stima dei modelli precedenti, ed indica che i luoghi con un grande ammontare di addetti nell'industria turistica tendono a crescere meno che la media.

Riguardo alle variabili di urbanizzazione, population è statisticamente significativa con un coefficiente $\beta=0.574$, diversity (inversa dell'indice $\mathrm{HH}$ calcolato su tutte le industri a livello di due codici ateco) è negativa $\beta=-0.522$. Questo può essere interpretato come una positiva relazione tra la dimensione urbana (infrastrutture e altre amenità), che cresce più lentamente nelle città con una più ampia struttura economica diversificata. L'esistenza di una relativa specializzazione nell'industria è positiva ed è statisticamente significativa, ma il coefficiente risulta molto piccolo $(\beta=0,03)$.

I coefficienti seguono gli stessi segni della stima del paragrafo precedente, ma cambia l'intensità dell'elasticità. Nei sistemi locali i coefficienti risultano essere più grandi. Questo può confermare l'ipotesi presentate nella sezione precedente. I sistemi locali del lavoro risultano essere una più corretta unità di analisi e non la municipalità.

\subsubsection{Modelli spaziali}

Innanzitutto presentiamo il test Moran globale per la presenza di spillovers. Il grafico sottostante mostra un valore per la I di Moran di 0,26 ed evidenzia l'esistenza di effetti spaziali tra sistemi locali adiacenti. Questo indica che esiste un effetto spaziale nei sistemi locali turistici adiacenti. Infine, Non si registra presenza di outliers.

Figura 5.3: I di Moran;

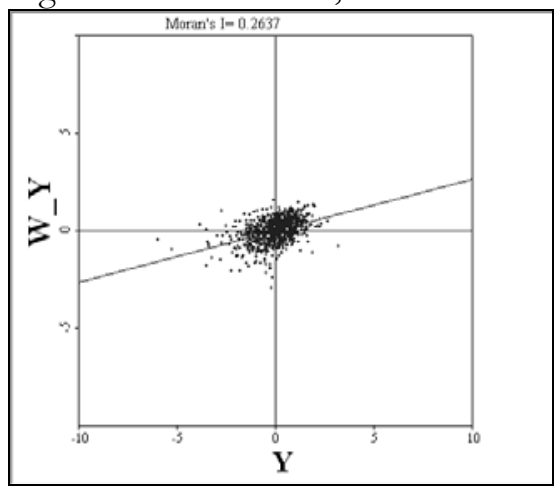

Fonte: nostra elaborazione con GEODA su dati Istat 1991-2001;

I test spaziali (LM Lag e LM error) suggeriscono l'esistenza di qualche tipo di autocorrelazione spaziale. Dato che il test nel modello dell'errore è più grande di quello del ritardo (lag) quello dell'errore può essere più idoneo alla stima (Anselin, 1992) ${ }^{121}$.

Iniziamo a stimare un modello che include il ritardo spaziale con variabili esogene (lag - cross regressive spatial model) (tabella 5.7). Dato che tutte le variabili sono in logaritmi e che la matrice dei contatti spaziali è standardizzata per righe, i coefficienti spaziali possono essere interpretati come elasticità dirette. In ogni modo ogni coefficiente spaziale è statisticamente significativo (tabella 5.7, colonna 3) e l'autocorrelazione spaziale

120 Se si effettua un regressione separata, il coefficiente risulta positivo ed indica che, l'esistenza di una dimensione di piccola impresa è relazionato ad una migliore performance nella crescita dell'occupazione nella filiera.

121 Abbiamo anche effettuato altri test forniti da SpaceStat 1.91 (Moran's I, KR, Robus LM error and Lag, and SARMA). 
continua ad essere principalmente catturata dal test $\mathrm{LM}^{122}$.

I modelli di ritardo spaziale e di errore spaziale sono stimati tramite la massima verosimiglianza (ML) ed i parametri spaziali sono statisticamente significativi $\varrho=0,26$ (lag) e $\lambda=0.76$ (errore). I test Akaike e Schwartz confermano che il modello di errore spaziale è maggiormente idoneo alla stima del modello di ritardo spaziale e del modello non spaziale.

Gli effetti dei coefficienti non spaziali sono più grandi di quelli stimati con il municipio. Questi confermano le ipotesi dell'esistenza di una sorte di trasmissione di shocks stocastici attraverso lo spazio (Anselin, 1988) o che i sistemi locali del lavoro e non la municipalità sono la corretta unità di analisi.

Figura 5.4: le reti di SLL e cluster map;

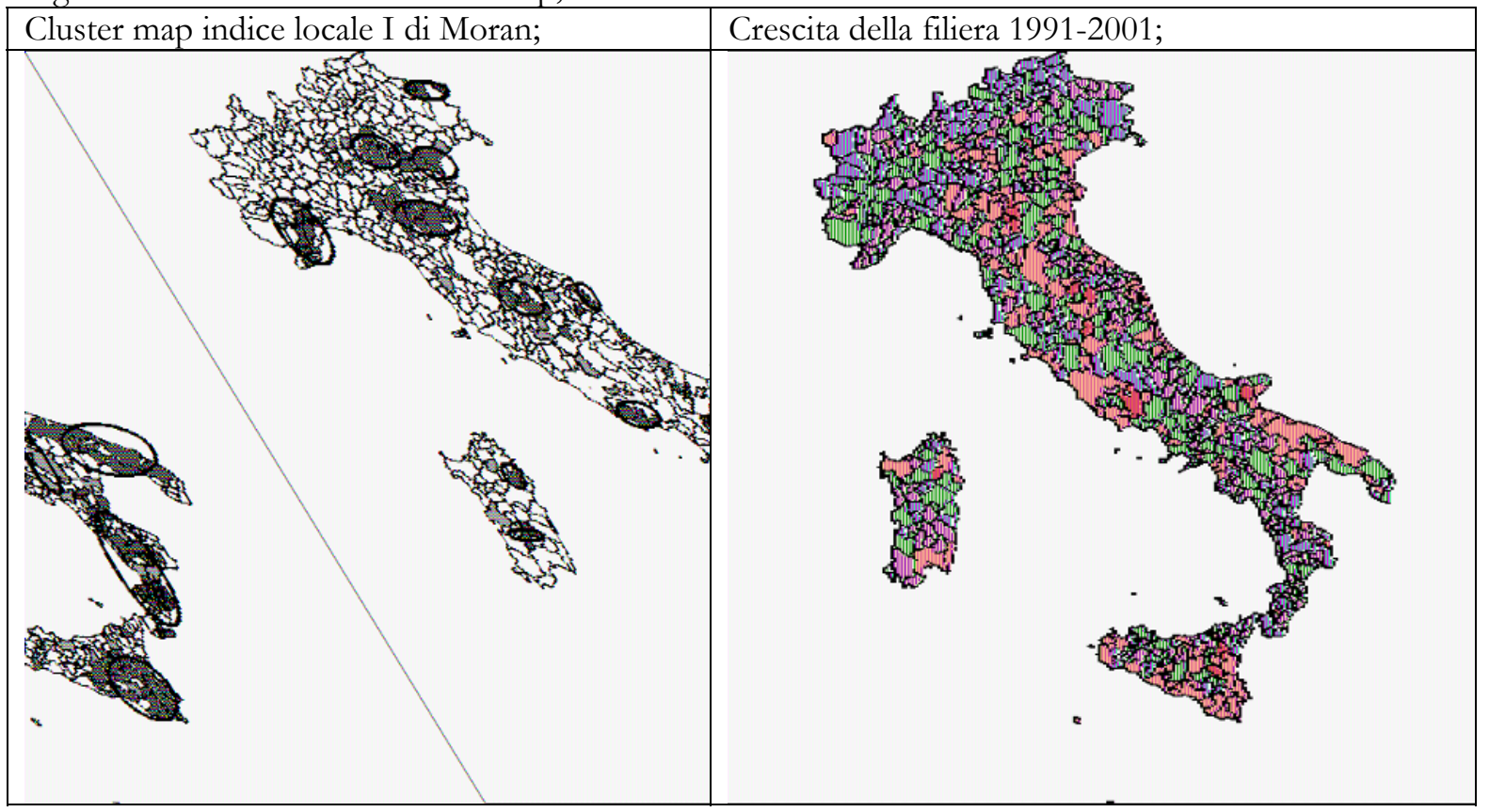

Fonte: ns. elaborazione su dati Istat;

Infine presentiamo la cluster map (figura 5.4) che indica le reti di SLL più significative per l'indice I di Moran locale. Si vede come i sistemi turistici che sono cresciuti maggiormente registrano un indice di autocorrelazione spaziale più alto con i sistemi limitrofi. Questo conferma che una migliore performance dei sistemi di impresa si diffonde a livello territoriale e supera i confini dei sistemi locali.

122 Dato che questo modello è collineare (condition number $=99.94$ ). Abbiamo stimato il modello includendo solo una variabile alla volta. In ogni modo, non si è registrato effetti spaziali significativi. 
Analisi delle fonti di competitività dei SLT

Tabella 5.6: Regressioni per gli effetti di localizzazione, urbanizzazione relazioni input-output;

\begin{tabular}{|c|c|c|c|c|c|c|}
\hline \multicolumn{3}{|c|}{ LOCALISATION } & \multicolumn{2}{|c|}{ URBANISATION } & \multicolumn{2}{|c|}{ INPUT-OUTPUT } \\
\hline Constant & $\begin{array}{l}0,0724 * * \\
(0,03293)\end{array}$ & $\begin{array}{c}0,0886 \\
(0,2289)\end{array}$ & Constant & $\begin{array}{c}-2,8555^{* * *} \\
(0,0000)\end{array}$ & Constant & $\begin{array}{c}-0,4069^{* * *} \\
(0,0000)\end{array}$ \\
\hline Ln Small firm & $\begin{array}{c}0,5667^{* * *} \\
(0,0000)\end{array}$ & $\begin{array}{c}0,5636 * * * \\
(0,0000)\end{array}$ & Ln Population & $\begin{array}{c}0,3178^{* * *} \\
(0,0000)\end{array}$ & Agriculture & $\begin{array}{c}-0,0895^{* * *} \\
(0,0000)\end{array}$ \\
\hline Employment & $\begin{array}{c}0,0967^{* * *} \\
(0,0000)\end{array}$ & $\begin{array}{c}-0,8305^{* * *} \\
(0,0000)\end{array}$ & Ln Diversity & $\begin{array}{c}-0,2645^{* * *} \\
(0,1387)\end{array}$ & Manufactures & $\begin{array}{l}-0,0169 \\
(0,2164)\end{array}$ \\
\hline LQF & $\begin{array}{c}-0,8243 * * * \\
(0,0000)\end{array}$ & $\begin{array}{c}0,0909 * * * \\
(0,0000)\end{array}$ & Ln Education & $\begin{array}{c}-0,1011^{* *} \\
(0,0303)\end{array}$ & Building & $\begin{array}{l}-0,0045 \\
(0,7836)\end{array}$ \\
\hline Filiere & $\begin{array}{c}0,1846^{* * *} \\
(0,0000)\end{array}$ & $\begin{array}{c}0,1662^{* * *} \\
(0,0000)\end{array}$ & & & Retail & $\begin{array}{c}0,0276^{* *} \\
(0,0406)\end{array}$ \\
\hline PACA dummy & & $\begin{array}{c}0,1322^{* * *} \\
(0,0000)\end{array}$ & & & Services & $\begin{array}{c}0,0156 \\
(0,2944)\end{array}$ \\
\hline $\mathrm{R} 2$ & 0,7253 & 0,7306 & $\mathrm{R} 2$ & 0,1258 & $\mathrm{R} 2$ & 0,1187 \\
\hline R2-adj & 0,7239 & 0,7289 & R2-adj & 0,1225 & R2-adj & 0,1130 \\
\hline AIC & 847,462 & 834,182 & AIC & 1753,16 & AIC & 1763,52 \\
\hline SC & 870,786 & 862,168 & SC & 1771,82 & SC & 1791,51 \\
\hline SIG-SQ & 0,1703 & 0,1670 & SIG-SQ & 0,5423 & SIG-SQ & 0,5467 \\
\hline$\underline{\mathrm{SIG}-\mathrm{SQ}(\mathrm{ML})}$ & 0,4127 & 0,4087 & SIG-SQ(ML) & 0,7364 & SIG-SQ(ML) & 0,7394 \\
\hline $\mathrm{CN}$ & 14,9918 & 15,7598 & $\mathrm{CN}$ & 59,1420 & $\mathrm{CN}$ & 10,5240 \\
\hline Jarque-Bera & $22,6237^{* * *}$ & 21,8631 *** & Jarque-Bera & $350,2193^{* * *}$ & Jarque-Bera & $415,5416^{* * *}$ \\
\hline Koenker-Bassett & $85,4547 * * *$ & $87,0730^{* * * * *}$ & Koenker-Bassett & $27,1739 * * *$ & Koenker-Bassett & $19,0272^{* * *}$ \\
\hline LM error & $269,6075^{* * *}$ & $276,1947 * * *$ & LM error & $78,6559 * * *$ & LM error & $93,5274 * * *$ \\
\hline LM lag & $25,8703^{* * *}$ & $30,1989 * * *$ & LM lag & $83,5426 * * *$ & LM lag & $99,9690 * * *$ \\
\hline OBS & 784 & 784 & OBS & 784 & OBS & 784 \\
\hline
\end{tabular}

P-values in parentesi. ${ }^{*} \mathrm{p}<0,1 ; * * \mathrm{p}<0,05 ; * * * \mathrm{p}<0,01$. 


\section{Capitolo quinto}

Tabella 5.7: Risultati modelli spaziali;

\begin{tabular}{|c|c|c|c|c|c|}
\hline & OLS & OLS & OLS & ML LAG GHET & ML ERROR GHET \\
\hline \multirow[t]{2}{*}{ Constant } & $-2,2559 * * *$ & $-2,2248^{* * *}$ & $-1,8831 * * *$ & $-2,1068 * * *$ & $-3,5017 * * *$ \\
\hline & $(0,0000)$ & $(0,0000)$ & $(0,0000)$ & $(0,0000)$ & $(0,0000)$ \\
\hline \multirow[t]{2}{*}{ Ln Small firm 1991} & $-0,0517$ & $-0,05759$ & $-0,2026^{* * *}$ & $-0,0257$ & $-0,2842 * * *$ \\
\hline & $(0,3770)$ & $(0,3256)$ & $(0,0000)$ & $(0,6489)$ & $(0,0000)$ \\
\hline \multirow[t]{2}{*}{ Ln Employment 1991} & $-0,5014 * * *$ & $-0,5072 * * *$ & $-0,7401 * * *$ & $-0,4784 * * *$ & $-0,7993 * * *$ \\
\hline & $(0,0000)$ & $(0,0000)$ & $(0,0000)$ & $(0,0000)$ & $(0,0000)$ \\
\hline \multirow[t]{2}{*}{ Ln Filiere 1991} & $0,3550^{* * *}$ & $0,3450^{* * *}$ & $0,1897 * * *$ & $0,3294 * * *$ & $0,1531 * * *$ \\
\hline & $(0,0000)$ & $(0,0000)$ & $(0,0000)$ & $(0,0000)$ & $(0,0000)$ \\
\hline \multirow[t]{2}{*}{ Ln Population 1991} & $0,5738^{* * *}$ & $0,5752^{* * *}$ & $0,7635^{* * *}$ & $0,5294 * * *$ & $0,8025^{* * *}$ \\
\hline & $(0,000)$ & $(0,0000)$ & $(0,0000)$ & $(0,0000)$ & $(0,0000)$ \\
\hline \multirow[t]{2}{*}{ Ln Diversity1991 } & $-0,5221 * * *$ & $-0,5392 * * *$ & $-0,0796$ & $-0,4255^{* * *}$ & $0,0607 * * *$ \\
\hline & $(0,0000)$ & $(0,0000)$ & $(0,5516)$ & $(0,0000)$ & $(0,0000)$ \\
\hline \multirow[t]{2}{*}{ Ln Manufactures 1991} & $-0,0324 * * *$ & $-0,0314 * * *$ & $-0,0296 * * *$ & $-0,0277 * * *$ & $-0,0201 * * *$ \\
\hline & $(0,0002)$ & $(0,0004)$ & $(0,0001)$ & $(0,0000)$ & $(0,0000)$ \\
\hline \multirow[t]{2}{*}{ PACA dummy } & & $0,0816^{*}$ & $0,1744^{* * *}$ & $0,1075^{* * *}$ & $0,1822^{* * *}$ \\
\hline & & $(0,0539)$ & $(0,0000)$ & $(0,0076)$ & $(0,0000)$ \\
\hline \multicolumn{6}{|l|}{$W^{*}$ Ln Small firm 1991} \\
\hline \multirow[t]{2}{*}{ W * Ln Labour 1991} & & & $0,4454 * * *$ & & \\
\hline & & & $(0,0000)$ & & \\
\hline \multirow[t]{2}{*}{ W * Ln Filiere 1991} & & & $0,3678^{* * *}$ & & \\
\hline & & & $(0,0000)$ & & \\
\hline \multirow[t]{2}{*}{ W * Ln Population ${ }_{1991}$} & & & $-0,3678^{* * *}$ & & \\
\hline & & & $(0,0000)$ & & \\
\hline \multirow[t]{2}{*}{ W * Ln Diversity 1991} & & & $-0,5656^{* * *}$ & & \\
\hline & & & $(0,0006)$ & & \\
\hline W * Ln Manufactures 1991 & & & - & & \\
\hline \multirow[t]{2}{*}{ W * PACA Dummy } & & & $-0,1801 * * *$ & & \\
\hline & & & $(0,00053$ & & \\
\hline \multirow[t]{2}{*}{$\varrho$} & & & & $0,2628 * * *$ & \\
\hline & & & & $(0,0000)$ & \\
\hline \multirow[t]{2}{*}{$\lambda$} & & & & & $0,7687^{* * *}$ \\
\hline & & & & & $(0,0000)$ \\
\hline R2 & 0,5793 & 0,5813 & 0,6757 & 0,6147 & 0,7720 \\
\hline R2-adj & 0,5760 & 0,5775 & 0,6707 & & \\
\hline SQ Corr & & & & - & - \\
\hline $\mathrm{AIC}$ & 1185,77 & 1184,01 & 993,665 & 1131,78 & 833,802 \\
\hline $\mathrm{SC}$ & 1218,42 & 1221,33 & 1054,3 & 1173,76 & 871,1172 \\
\hline $\mathrm{CN}$ & 45,7983 & 47,0237 & 99,9315 & & \\
\hline Jarque-Bera & $36,70 * * *$ & $33,9451 * * *$ & & & \\
\hline Koenker-Bassett & $130,3916^{* * *}$ & $127,4418^{* * *}$ & & & \\
\hline WALD HET (1) / BP HET (2) & & & $160,1079 * * * *$ & $175,3642 * * *(2)$ & $162,9462^{* * *}(2)$ \\
\hline LR HET & & & & $54,2319 * * *(2)$ & $350,2111 * * * *(2)$ \\
\hline LM error & $258,5542 * * *$ & $270,0966 * * *$ & $233,0330^{* * *}$ & & \\
\hline LM lag & $59,1838^{* * *}$ & $62,3929 * * *$ & $250,2439 * * *$ & & \\
\hline OBS & 784 & 784 & 784 & 784 & 784 \\
\hline
\end{tabular}




\subsubsection{Regimi Spaziali}

Infine verifichiamo la performance dei modelli sulle diverse tipologie di sistemi locali turistici utilizzando i regimi spariali, come nella sezione precedente. Ricordiamo che questo procedimento è stato presentato da Anselin (1988 e 1992). Utilizzando questa struttura possiamo stimare il modello OLS e il modello degli errori spaziali nella forma usuale: $y^{*}=X^{*} \beta^{*}+u^{*}$. Aggreghiamo i sistemi locali del gruppo 1, 3 e 5 dato che la stima per sistemi locali riduce il numero di osservazioni e provoca problemi di significatività.

I risultati (tabella 5.8) mostrano alcune interessanti caratteristiche. Il modello ha una bassa performance su tutti $\mathrm{i}$ cluster.

Il Test di Chow-Wald $\left(\chi^{2}\right)$ conferma inoltre che ci sono dei problemi di instabilità strutturale nel modello considerato per intero, principalmente associato alle variabili dei fornitori della filiera ${ }^{123}$.

Infine, i regimi spaziali applicati ai sistemi locali perdono di significatività e non riescono a catturare un buon livello di $\mathrm{R}^{2}$. Il massimo registrato arriva intorno al 13\% anche se il test sull'errore arriva al 30\%. Questo perché la numerosità del campione diminuisce vertiginosamente data l'aggregazione per sistemi locali del lavoro e all'analisi dei soli SLT.

123 Si evidenzia tuttavia la presenza di una forte collinearità. 
Tabella 5.8: Regimi spaziali;

\begin{tabular}{|c|c|c|c|c|c|c|}
\hline & \multicolumn{3}{|c|}{ OLS } & \multicolumn{3}{|c|}{ ML ERROR } \\
\hline & C1 & $\mathrm{C} 2$ & C3 & C1 & C2 & C3 \\
\hline Constant & $\begin{array}{l}-0.2573 \\
(0.8605)\end{array}$ & $\begin{array}{c}-3.14026^{* * *} \\
(0.0000)\end{array}$ & $\begin{array}{l}-1.7797 \\
(0.1796)\end{array}$ & $\begin{array}{l}-1.0553 \\
(0.3419)\end{array}$ & $\begin{array}{c}-3.51542^{* * *} \\
(0.0000)\end{array}$ & $\begin{array}{c}-1.76975 \\
(0.1083)\end{array}$ \\
\hline Ln Employment 1991 & $\begin{array}{c}-0.5410^{* * *} \\
(0.0005)\end{array}$ & $\begin{array}{c}-0.5284^{* * *} \\
(0,0000)\end{array}$ & $\begin{array}{c}-0.5523^{* * *} \\
(0.0005)\end{array}$ & $\begin{array}{c}-0.6423^{* * *} \\
(0.0000)\end{array}$ & $\begin{array}{c}-0.7363^{* * *} \\
(0.0000)\end{array}$ & $\begin{array}{c}-0.7721^{* * *} \\
(0.0000)\end{array}$ \\
\hline Ln Filiere $_{1991}$ & $\begin{array}{c}0.1294 \\
(0.6130)\end{array}$ & $\begin{array}{c}0.3944^{* *} \\
(0.0031)\end{array}$ & $\begin{array}{c}0.5365 \\
(0.1125)\end{array}$ & $\begin{array}{l}0.1512 \\
(0.5017)\end{array}$ & $\begin{array}{c}0.2517^{* *} \\
(0.0455)\end{array}$ & $\begin{array}{c}0.2980 \\
(0.3177)\end{array}$ \\
\hline Ln Population 1991 & $\begin{array}{c}0.4171^{* *} \\
(0.0196)\end{array}$ & $\begin{array}{c}0.6190^{* * *} \\
(0,0000)\end{array}$ & $\begin{array}{c}0.5217^{* *} \\
(0.0132)\end{array}$ & $\begin{array}{c}0.6138^{* * *} \\
(0.0002)\end{array}$ & $\begin{array}{c}0.8046 * * * \\
(0.0000)\end{array}$ & $\begin{array}{c}0.7083^{* * *} \\
(0.0004)\end{array}$ \\
\hline PACA Dummy & $\begin{array}{l}0.1651 \\
(0.4705)\end{array}$ & $\begin{array}{c}-0.05307 \\
(0.6994)\end{array}$ & $\begin{array}{l}-0.1619 \\
(0.4695)\end{array}$ & $\begin{array}{c}0.2234 \\
(0.3427)\end{array}$ & $\begin{array}{c}0.0654 \\
(0.6159)\end{array}$ & $\begin{array}{l}-0.0866 \\
(0.6880)\end{array}$ \\
\hline$\lambda$ & & & & $\begin{array}{c}0.5177^{* * *} \\
(0.0000) \\
\end{array}$ & & \\
\hline $\mathrm{R} 2$ & 0.1257 & & & 0.2929 & & \\
\hline R2-adj & 0.1098 & & & & & \\
\hline SQ Corr & & & & 0.1252 & & \\
\hline AIC & 1775.29 & & & 1625.65 & & \\
\hline SC & 1845.25 & & & 1695.62 & & \\
\hline SIG-SQ & 0.5529 & & & 0.4216 & & \\
\hline SIG-SQ(ML) & 0.5424 & & & & & \\
\hline $\mathrm{CN}$ & 52.5385 & & & & & \\
\hline Jarque-Bera & $378.3759 * * *$ & & & & & \\
\hline Chow - Wald & $2.5550 * *$ & & & $29.5034^{* *}$ & & \\
\hline Koenker-Bassett & $34.0789 * * *$ & & & & & \\
\hline Breusch-Pagan & & & & $88.1664 * * *$ & & \\
\hline Spatial BP & & & & n.s. & & \\
\hline LM ERROR & $155.0692^{* * *}$ & & & & & \\
\hline$\underline{\mathrm{LM}} \mathrm{LAG}$ & $146.4155^{* * *}$ & & & & & \\
\hline OBS & $35+17+4$ & 157 & 56 & $35+17+4$ & 157 & 56 \\
\hline
\end{tabular}

\subsubsection{Conclusioni del secondo studio}

L'obiettivo di questo capitolo è stato quello di misurare la fonte della competitività dei sistemi turistici locali identificati nel capitolo precedente. In particolare in questo secondo studio abbiamo valutato le fonti della competitività per i sistemi locali del lavoro: sia a livello di singolo sistema locale che a livello di spillovers tra sistemi locali. In relazione a questo argomento l'ipotesi principale è stata quella di differenziare tra due fonti principali: risorse naturali ed economie esterne. Utilizzando il framework già presentato nel terzo capitolo di questa tesi si è testato la triade marshalliana per le economie distrettuali e la classificazione di Hoover (1937) si è quindi suddiviso l'analisi in economie di localizzazione e di urbanizzazione.

Nel quarto capitolo abbiamo individuato i sistemi locali turistici italiani, verificando l'applicabilità di modelli econometrici spaziali relativamente ai diversi tipi di sistemi turistici locali identificati tramite la cluster analysis. I test spaziali (LM Lag e LM error) suggeriscono l'esistenza di qualche tipo di autocorrelazione spaziale. I parametri spaziali sono significativi (con un $\varrho=0.26$ sul lag e $\lambda=0.76$ sull'errore) e quindi si presume che esista un network anche 
a livello dei sistemi locali del lavoro limitrofi. Da questo punto di vista questi risultano essere una più idonea unità di analisi territoriale come nell'ipotesi di Sforzi (1997a) e Menghinello (2002).

I modelli dei regimi spaziali, come nella parte precedente registrano della buone performance sui cluster Città d'arte e Sistemi turistici specializaati (in una sola parte della filiera). Al contrario solo pochi coefficienti del cluster dei distretti turistici risultano statisticamente significativi (occupazione e popolazione). Dato che il modello dei regimi spaziali proviene principalmente da studi di economia urbana e risulta totalmente soddisfacente per medie e grandi città, ma non ottiene lo scopo di raggiungere per i sistemi locali un buon livello di significatività.

In breve i tassi di crescita più alti sono associati alla presenza locale di tutte le fasi della filiera turistica. Infatti questa variabile riesce a spiegare circa il $30 \%$ della varianza e conferma che la filiera proposta risulta idonea allo studio del fenomeno in questione anche a livello di sistema locale, anche se l'importanza di tale variabile risulta ridotta, in confronto all'analisi a livello municipale.

Importanti implicazioni emergono da tali risultati per i policy makers e suggeriscono una maggiore attenzione per promuovere lo sviluppo di ogni anello della filiera turistica. Inoltre i coefficienti della stima per i sistemi locali risultano più grandi e confermano l'ipotesi che il sistema locale sia la più idonea unità territoriale di analisi. Infine, il risultato positivo di correlazione spaziale e la positività del coefficiente dell'errore dimostrano come la competitività di un luogo è correlato alla competitività dei luoghi limitrofi. Per concludere il test indicativo della presenza di risorse naturali è soddisfacente anche se non privo di critiche. Il coefficiente relativo al PACA è positivo, ma una maggiore attenzione alla costruzione del database sulle risorse naturali è necessario per gli studi futuri ${ }^{124}$.

In relazione ad ulteriori sviluppi della ricerca, il concetto marshalliano di economie esterne deve essere necessariamente approfondito in modo da costruire un modello che riesce a costruire l'effetto delle economie di localizzazione costituite da mano d'opera qualificata, fornitori specializzati e spillovers di conoscenza. L'ultimo concetto è legato alla "industrial atmosphere" marshalliana che come in un distretto industriale è molto difficile da catturare tramite analisi quantitative. Alcuni autori, in particolare, (Becattini, Bellandi, Dei Ottati, Sforzi 2001; Lazzeretti 2003) hanno cercato di spiegare come questo fattore sia difficilmente quantificabile e venga espresso tramite analisi qualitative.

\subsection{CONCLUSIONI}

Lo scopo di questo capitolo è stato misurare i vantaggi competitivi dei SLT, suddivisi in base a risorse naturali, economie esterne e spillovers. Abbiamo stimato le diverse performance dei sistemi locali turistici in rete tra network di città e tra network di sistemi locali.

La stima dei modelli econometrici è stata elaborata tramite due software SpaceStat e Geoda sviluppati da Anselin $(1988,1992,2002)$ per le analisi in Spatial Econometrics. Le unità territoriali (comuni e sistemi locali) sono state analizzate in base alle caratteristiche di quelle limitrofe., cercando di misurare il grado di influenza e di spillover tra località turistiche vicine. L'ipotesi principale è stata focalizzarsi sulle economie esterne dei sistemi di produzione, utilizzando la classificazione presentata nel terzo capitolo che le suddivide in economie di

${ }^{124}$ Si rimanda a tal fine all'esame della mappatura del patrimonio artistico e culturale nella Regione Toscana effettuato dall'IRPET in base alle stelle dei comuni registrate nella guida del Touring Club Italia (Bacci, 2002). 
localizzazione, urbanizzazione ${ }^{125}$ e rete. Si è, poi, evidenziato il peso più rilevante delle economie di localizzazione e delle economie di rete.

Si è stati in grado inoltre di confermare che il sistema locale è una più idonea unità di analisi territoriale nell'industria turistica come nell'ipotesi di Sforzi (1997a) e Menghinello (2002), rispetto alla municipalità.

In sintesi, si è verificato come i tassi di crescita più alti dei SLT sono associati alla presenza locale di tutte le fasi della filiera. Si è dimostrato come questa variabile riesca a spiegare un alto livello di varianza in entrambi gli studi, confermando l'adeguatezza delle attività incluse. A livello di sistema locale l'importanza di tale variabile è risultata ridotta, in confronto all'analisi a livello municipale, dove si è registra, invece, una maggiore importanza delle reti territoriali.

Importanti implicazioni sono emerse da tali risultati sia a livello di politiche che di gestione. In particolare si rileva come non sia sufficiente utilizzare un approccio sistemico allo studio delle destinazioni turistiche, ma come sia necessario promuovere lo sviluppo di ogni singolo anello della filiera e focalizzarsi su reti territoriali di destinazioni e su reti di reti.

Questo capitolo empirico risulta una robusta misurazione dei vantaggi competitivi di sistemi di imprese e si inserisce nel dibattito internazionale nella stima delle externalities (Glaeser et al. 1992; Rosenthal e Strange, 2002; Paci ed Usai, 1999). Questa prima analisi ha fornito risultati soddisfacenti con buoni livelli di significatività statistica. Inoltre la stima dei vantaggi competitivi tra reti di comuni e reti di sistemi locali risulta un contributo originale per l'industria turistica. La rilevanza della correlazione spaziale individuata sottolinea come ancor di più nel turismo sia necessario focalizzarsi su reti di destinazioni e allargare la partecipazione degli attori locali. Questo studio al quale sono necessarie ulteriori approfondimenti e perfezionamenti della base di dati risulta una solida base per la stima dei vantaggi delle destinazioni turistiche italiane e mette in risalto chiari indirizzi di policy e gestione.

Infine, si è verificata l'importanza della presenza di risorse naturali ed artistiche $(P A C A)$. La stima della variabile è risultata significativa e soddisfacente anche se non priva di critiche. Da questo punto di vista il patrimonio artistico, culturale ed ambientale, di cui il territorio italiano è diffusamente dotato, assume un ruolo importante nello sviluppo locale se opportunamente valorizzato e gestito da un consono sistema di imprese integrato a livello territoriale.

\footnotetext{
125 Si rimanda per questo punto necessariamente al capitolo due di questo scritto.
} 


\section{RIFLESSIONI CONCLUSIVE}

Questo lavoro si inserisce nell'attuale dibattito sui differenziali di competitività di imprese, settori e sistemi locali, con particolare riferimento al ruolo della globalizzazione e dei vantaggi di tipo locale. In questo contesto socio-economico, in cui la competizione di nazioni e di imprese è sempre più legata ai vantaggi dei luoghi, ci siamo interrogati su come potrà evolvere e competere l'industria turistica in base agli ultimi sviluppi.

In questa ottica, non risulta più sufficiente analizzare il turismo come un insieme di attività, né focalizzare l'attenzione su macro aree geografiche (nazioni o regioni). I luoghi con le proprie caratteristiche specifiche ed uniche possono essere la base per costituire differenziali importanti per una maggior competitività a livello nazionale (tra luoghi) ed internazionale (tra nazioni) le cui peculiarità non possono essere sottovalutate.

Gli approcci sistemici al turismo, di crescente interesse negli ultimi anni, sottolineano l'importanza di un focus a 360 gradi su tutte le attività e specificità di una destinazione turistica. Ci siamo inseriti in questo contesto con l'obiettivo di verificare quali sono i luoghi turistici italiani e come competono tra di loro. In particolare, siamo partiti considerando sistemi locali di imprese specializzate in attività turistiche in senso ampio, al fine di verificarne la capacità di valorizzare il patrimonio artistico, culturale ed ambientale diffuso su tutto il territorio nazionale.

Alla fine di questo studio, cerchiamo di trarre alcune considerazioni di sintesi in relazione alle due questioni di fondo che ci eravamo prefissi di affrontare:

a) Identificare i luoghi turistici in Italia;

b) Analizzare e misurare la loro competitività.

\section{a) Identificazione dei SLT}

Innanzitutto, per giungere ad un'identificazione dei sistemi locali turistici in Italia, l'impiego dell'approccio distrettuale (industriale-manifatturierio) è risultato utile per interpretare l'industria turistica, costituita da un tessuto di piccole e medie imprese e da luoghi territorialmente e socialmente definiti.

Riguardo all'unità di riferimento, l'utilizzo dei sistemi locali del lavoro ha permesso di focalizzarsi su unità territoriali costruite in base alle reali caratteristiche socioeconomiche dei luoghi e non in base a confini meramente amministrativi.

La filiera proposta ha mostrato una buona applicabilità alle analisi e le attività incluse si sono dimostrate coerenti con lo studio dell'industria turistica. I risultati raggiunti hanno rilevato che è essenziale focalizzarsi, per lo studio di una destinazione, su approcci di tipo sistemico, ma sopratutto che è necessario porre attenzione alla promozione, gestione e sviluppo di ogni singolo anello della filiera turistica di una località.

La qualità dell'esperienza del visitatore, il viaggio, dipende sempre di più non solo dalle caratteristiche dell'attrazione principale, ma anche dalla qualità ed efficienza di servizi complementari come alberghi, ristoranti, trasporti, etc. La debolezza di un anello della filiera, come evidenziato, può influenzare ed indebolire l'offerta turistica delle imprese che costituiscono il sistema e di conseguenza quella complessiva del luogo.

A partire dalla filiera allargata si sono identificati i sistemi locali turistici italiani ed utilizzando ArcView si è sviluppata un'ampia gamma di rappresentazioni cartografiche di notevole interesse come supporto all'analisi economica. Le mappe hanno rappresentato 
una nuova geografia dell'Italia costituita dai SLT che risulta coincidente con le destinazioni turistiche italiane.

Questa parte si configura quindi come un contributo che amplia e approfondisce lo stato dell'arte sull'individuazione dei luoghi turistici in Italia principalmente da tre punti di vista. Il primo si riferisce all'unità di analisi; i sistemi locali del lavoro si sono dimostrati un'idonea unità di riferimento territoriale al fine di analizzare un fenomeno che ha caratteristiche socio-economiche. Il secondo aspetto è relativo all'innovativo utilizzo di una filiera per lo studio di attività turistiche, sottolineando la necessità di una visone allargata del fenomeno che non sia solo limitata alle attività turistiche in senso stretto. Infine, la base di dati costruita ad hoc ed aggiornata al censimento 2001 ha permesso in primo luogo, di effettuare un'istantanea updated della situazione italiana ed in seconda istanza di sviluppare un esame dell'evoluzione delle destinazioni turistiche nell'ultimo decennio.

\section{b) La misurazione e l'analisi della competizione dei SLT}

Il secondo obiettivo era legato alla misurazione della competitività dei sistemi locali turistici in Italia. Lo studio è stato pertanto suddiviso in relazione alle fonti della competitività costituite da risorse naturali ed economie esterne (localizzazione, urbanizzazione, rete). Si è considerando in prima istanza i SLT come unità territoriali chiuse. Abbiamo, poi, superato questi confini territoriali al fine di analizzare i border effects e gli spillover tra sistemi limitrofi. Le economie esterne sono state quindi misurate in reti di comuni all'interno di un sistema locale e in reti di sistemi locali.

È stato dimostrato come non solo ci siano degli effetti spaziali tra comuni turistici all'interno dei sistemi locali, come d'altronde era prevedibile, ma soprattutto tra SLT limitrofi. Da questo punto di vista i sistemi locali del lavoro si sono confermati come la più idonea unità territoriale di analisi.

$\mathrm{Al}$ fine di una migliore gestione, pianificazione e promozione del turismo dei luoghi sarà necessario in futuro analizzare più approfonditamente il ruolo delle reti territoriali di destinazioni turistiche e dei collegamenti e sinergie tra reti.

I tassi di crescita più alti dei SLT sono associati alla presenza locale di tutte le fasi della filiera e questa variabile riesce a spiegare un alto livello di varianza in entrambi gli studi, confermando l'adeguatezza delle attività incluse.

I modelli di analisi urbana e regionale applicati a questa industria specifica si sono dimostrati efficaci ed $i$ risultati raggiunti si inseriscono in un più ampio dibattito internazionale sul ruolo delle externalities per lo studio della crescita di regioni e città. Questo approccio si configura inoltre come un contributo sostanziale data l'originalità della sua estensione al turismo.

Si è poi valutato la sinergia tra la struttura dell'offerta e le risorse naturali, cercando soluzioni al difficile problema di decomporre l'effetto di concentrazione proveniente dalle esternalità di un'industria, da quello delle risorse naturali della stessa industria. È emerso che il patrimonio artistico, culturale ed ambientale di cui il territorio italiano è diffusamente dotato, assume un ruolo importante nello sviluppo locale se opportunamente valorizzato e gestito da un consono sistema di imprese integrato a livello territoriale.

In sintesi questo lavoro rappresenta una solida base quantitativa di partenza per ulteriori approfondimenti, relativi all'identificazione dei sistemi turistici locali e alla misurazione della loro competitività, dato che le motivazioni che determinano la crescita di un luogo e lo sviluppo turistico sono sempre più al centro di un fervente dibattito. 


\section{B ibliografia}

\section{RIFERIMENTI BIBLIOGRAFICI}

AA.VV. (2002), "Undicesimo Rapporto sul Turismo", Mercury, Firenze.

Aci-Censis, Rapporto Turismo (2001) "I distretti turistici italiani: l'opportunità di innovare l'offerta", Roma, Censis servizi s.p.a.

Amin, A. e Goddard, J. (eds) (1986), "Technological change, industrial restructuring and regional development", London, Allend and Unwin.

Anselin L. (1988), "Spatial Econometrics: Methods and models", Kluwer Academic Publishers. the Neederlands.

Anselin, L. (1992), "SpaceStat tutorial: a workbook for using SpaceStat in the analysis of Spatial Data”, University of Illinois, Urban Champaign.

Anselin, L. (1998), "Gis research infrastructure for spatial analysis of real estate marketc, Journal of Housing research 9,113-33.

Anselin, L. (2004), "Spatial Analyses of Homicide with Areal Data", in corso di pubblicarione.

Anselin, L., Madden M. (1990), "New directions in regional analysis: integratedand multiregional approaches", NewYork, London, BelHaven, Press.

Antonioli Corigliano M. (1999), "Strade del vino ed enoturismo. Distretti turistici e vie di comunicazione", Milano, Franco Angeli.

Arrow, K.J. (1962), “The Economic implications of learning by doing”, Review of Economics Study, 29, 155-73.

Ashworth, G., (1991) "The historic cities of Groningen: which is sold to whom'?" in Marketing Tourism and Places, (a cura di) G. Ashworth e B. Goodall. Routledge, London

Aydalot, Ph., (1986), (éd.), "Milieux Innovateurs en Europe”, Paris, GREMI.

Bacci L. (2002), "Sistemi Locali in Toscana. Modelli e percorsi Territoriali dello sviluppo regionale", IRPET, Franco Angeli, Milano.

Bacci L., e Cavalieri A., (1998), "I sistemi Turistici nello sviluppo economico della Toscana", IRPET.

Baptista R. (1998), "Clusters, innovation and growth: A survey of the literature" in Swann P., Prevenzer M., Stout D (1998), "The Dynamics of Industrial Clustering", Oxford University Press.

Becattini G. (1990), "The Marshallian industrial district as a socioeconomic notion” in Pyke F., Becattini G., e Sengenberger W., (eds.) "Industrial Districts and Inter-firm co-operation, in Italy", ILO Geneva.

Becattini G., (1979), "Dal "settore industriale" al "distretto industriale". Alcune considerazioni sull'unità d'indagine dell'economia industriale", L'industria, n. 1

Becattini G., (1987), (a cura di) "Mercato e Forze locali: il distretto industriale", Bologna, Il Mulino.

Becattini G., (2000), "Il distretto Industriale“, Rosemberg \& Sellier, Torino.

Becattini G., Bellandi M., Dei Ottati G. e Sforzi F. (a cura di), (2001), "Il caledoscopio dello sviluppo locale", Rosemberg \& Sellier, Torino.

Becattini G., Sforzi F., (2002), (a cura di), "Lezioni sullo sviluppo locale", Rosemberg \& Sellier, Torino.

Becheri E., (2003), "I sistemi turistici locali: uno strumento superfluo", in Rivista del Turismo, 1, 2003.

Bellandi (1995), "Economie di scala e organizzazione industriale”, Franco Angeli editore, Milano, 


\section{B ibliografia}

1995.

Bellandi (2003), "Mercati industrie e luoghi di piccola e grande impresa", Bologna, Il Mulino

Bellandi M. e Sforzi F., (2003), "The multiple paths of local development" in Becattini G., Bellandi M., Dei Ottati G. e Sforzi F (2003) "From industrial districts to local development. An itinerary of research", Cheltenham (U.K.), Edward Elgar.

Bergman, E.M. e Fesser E.J(1999), "Industrial and regional clusters: concepts and comparative applications", The web book of regional science, Regional Research Institute, West Virginia University. (mwn.rri.wvu.edu/WebBook/).

Boix R., (2002), "Spatial Econometrics" Seminario al Dipartimento di Ingegneria Gestionale Politecnico di Milano 04/12/02, Milano.

Boix, R. (2003), "La medición de las economias externas en redes de ciudades" presentato alla XXIX Reunión de Estudios Regionales, Santander 27 y 28 de noviembre de 2003

Boix, R. (2004), "Redes de ciudades y externalidades", Investigaciones Regionales, no 4, p.5-27.

Bramanti, A. (1997), "Il Milieu Turistico del Garda Bresciano" in Commercio, 60, 1997.

Brusco S. (1989), "Piccole imprese e distretti industriali: una raccolta di saggi", Torino, Rosenberg \& Sellier, 1989.

Brusco S. e Solinas G., (1997) "Competitività e partecipazione: una proposta di politica del lavoro”, Bologna, Il Mulino, 1997

Brusco S. e Solinas G., (1997), Commissione Europea (DG V) "Competitività, partecipazione e distretti industriali: l'esperienza italiana”, Bruxelles, gennaio, 1998.

Brusco S., Solinas G., Minerva T. e Poli I., (2002) "Un automa cellulare per lo studio dei distretti industriali", in Politica economica , Fascicolo: 2, 147-192.

Buhalis, D., (2000), "Marketing the competitive destination of the future", in Tourism Management, vol. 21, pp. 97-116.

Buhalis, D., Cooper C., (1998), "Competition or co-operation: Small and Medium sized Tourism Enterprises at the destination", in E., Laws, B. Faulkner, G. Moscardo, (eds.), Embracing and managing change in Tourism, Routledge, London, pp.324-346.

Buhalis, D., Fletcher J. (1995), "Environmental impacts on tourism destinations: An economic analysis", in Coccosis, H., e Nijkamp, P., (eds), Sustainable Tourism Development, Avebury, England, pp.3-24.

Camagni, R. (1992), "Economia urbana. Principi e modelli teorici”, La Nuova Italia Scientifica. Roma.

Camagni, R. (1993), "Principi di Economia Urbana e territoriale", NIS, Roma.

Camagni, R. e Salone C., (1993), "Elemento per una teorizzazione delle reti di citta", in Camagni R. e Blasco G. (a cura di) "Le reti di citta': teoria, pollitiche e analisis nelll'area padana”, Franco Angeli, Milano.

Capone F., Boix R., (2004), "Sources of competitiveness in tourist local systems", Working Paper 04.08, Departament d'Economia Aplicada, Facultat de Ciències Econòmiques i Empresarial, Universidad Autonoma de Barcelona, Barcelona.

Capone F., (2004), "Regional competitivness in tourist local systems", relazione presentata al European Regional Science Association (ERSA) 2004 Congress, Oporto, Portogallo, 2529 Agosto 2004.

Casado Diaz J.M. (2000), "Local Labour Market Areas in Sain: A case study", Regional Studies, Vol . 34.9. pp.843-656.

Casarin, F., (1996), "Il marketing dei prodotti turistici. Specificità e varietä", Giappichelli Editore. 


\section{B ibliografia}

Cavalieri A. (1999), "Toscana e Toscane. Percorsi locali e identità regionali nello sviluppo economico", F. Angeli, Milano.

Cazes, G. (1994), “A Propos du Tourisme Urbain: Quelques Questions Pre'alables et De' rangeantes" in Cahiers Espaces 39:26-30.

Cazes, G., e F. Potier (1996), “Le Tourisme Urban””, Paris: Presses Universitaires de France.

Cazes, G., e F. Potier, (a cura di) (1998), "Le Tourisme et la Ville: Expe'riences Europe'enes", Paris: L'Harmattan.

Cerato M., Giannone M, Strano R., Primi F., (2003), "Per lo sviluppo del turismo: armonizzazione, valorizzazione e ruolo dei sistemi turistici locali" in AA.VV., "Dodicesimo Rapporto sul Turismo", Mercury, Firenze, pp. 691-706.

Chinitz B. (1961), "Contrast in agglomeration: New York and Pittsburgh", The American Economic Review, vol.51, Issue 2.

Clarke, K. C. (2001), "Getting Started with GIS”, Prentice Hall, University of California, Santa Barbara.

Conti, S., Malecki, E. e Oinas, P. (1995), "The industry enterprises and its environment spatial persprective", Aldershot, Avenbury.

Cooke, P., (2001), "Regional Innovation Systems, Clusters and the Knowledge Economy”, Industrial \& Corporate Change, 10, 945-974

Cooke, P., (2002), "Knowledge Economies", Routledge

Cooke, P., (eds.) (1989), “Localities” London: Allen and Unwin.

Costa, P., (2002), "Milieux effects and sustainable developments in a cultural quarter: the 'Barrio alto-Chiado' area in Lisbon" in "New economy and regional development" proceedings of IXth Meeting APDR, 27th - 29th June, 2002, Lisbon, Portugal.

Crescenzi F., (2002), "I sistemi informativi geografici e la qualità del dato statistico", paper presentato alla Sesta conferenza nazionale di Statistica, Roma, 6-8 Novembre 2002.

Cressie, N, (1999), "Spatial statistics applied to environmental problems in the life and medical sciences", Working Papers, Paris : INSEE, Institut national de la statistique et des études économiques (France).

Crouch I. G, Ritchie B. J. R. (1999), "Tourism, Competitivness, and Social Properity" in Journal of Business Research, vol. 44, pp. 137-152.

Dallari F., "Il distretto industriale tra spazio e territorio", dispense di Geografia Applicata, maggio 2002, http: \\www.dse.unibo.it \dallari.html.

De Lucio J.J., Herce J.A. e A. Goicolea (2002), "The effects of externalities on productivity growth in Spanish industriy", Regional Science and Urban Economics, $\mathrm{n}^{\circ} 32$, p.241-258.

Dei Ottati (1995), "Tra mercato e comunità: aspetti concettuali e ricerche empiriche sul distretto industriale", Franco Angeli, Collana Problemi dello sviluppo industriale, Milano.

Dei Ottati (2002), "Social concertation and local development: the case of industrial districts" in European planning studies, vol.10, n.4, 2002, pp.449-466.

Dei Ottati (2003), "Exit, voice, and the evolution of industrial districts: the case of the post-World War II economic develpment of Prato" in Cambridge Journal of Economics, vol.27, n.4, 2003, pp.501-522

Deidda S., Paci R. e Usai S., (2002), "Spatial externalities and local economic growth", Contributi di ricerca 02/06, CRENOS, Università degli Studi di Cagliari, Università degli Studi di Sassari.

Della Corte, V., (1999), "Dall'impresa alle forme di offerta complesse: le principali 


\section{B ibliografia}

problematiche strategiche ed organizzative di un sistema locale di offerta turistica", in Economia e Diritto del Terziario, 1, 1999.

Della Corte V., (2000), "La gestione dei sistemi locali di offerta turistica", Padova, CEDAM.

Dematteis, G. (1989), "Nuove forme di organizzazione territoriale" in Petsimeris P. (a cura di), "Le reti urbane tra decentramente e centralita", Franco Angeli, Milano.

Dematteis G., (2003), "Il modello Slot come strumento di analisi dello sviluppo locale", in Slot Quaderno 3, di Rosignoli C., Imarisio C. S. (a cura di), Bologna, Baskerville, pp-13-27.

Dewhurts, J. H. L., Mc Cann P. (2002), "A comparison of Measures of Industrial Specialization for travel to work areas in great britain, 1981-1997”, Regional Studies, Vol £6.5, pp. 541-551.

Di Meo A., (2002), "Marketing dell'ambiente e della cultura per lo sviluppo turistico del territorio", Editori di Comunicazioni, Lupetti, Milano.

Dillon, W. D. e M. Goldstein (1984), “Multivariate Analysis”, John Wiley \& Sons.

Doeringer P. B. e Terkla D. G. (1996), "Why do industries cluster?”, in Udo Staber, Norbert Schaefer e Basu Sharma (a cura di), Business Networks: Prospects for Regional Development, De Gruyter, New York.

Ellison, G. e Glaeser E., L.(1997), "Geographic concentration in U.S. manufacturing industries: a dartboard approach", Journal of Political Economy, vol. 107, n 5.

Ellison, G. e Glaeser E. (1999), "The geographic concentration of an industry: Does natural advantage explain agglomeration," American Economic Association Papers and proceedings $89,311-316$.

Enright M. J. (1992), "Why local cluster are the way to win the game", World link, 5 July/august, 24-25.

European Commission, (2000a), "Toward quality urban tourism-integrated quality management (iqm) of urban tourist destination", Luxemburg, E.U.

European Commission, (2000b), "Toward quality urban tourism- integrated quality management (iqm) of rural tourist destination", Luxemburg, E.U.

European Commission, (2000c), "Toward quality urban tourism- integrated quality management (iqm) of costal tourist destination", Luxemburg, E.U.

European Commission (2003), "Green Paper on Food Law”, Directorate General for Industry, Luxembourg.

European Network for SME Research (ENSR), (2003), "Regional Cluster in Europe", Luxembourg Office for Official Publications of the EC.

Faramondi, A, Piras, M.G., (2002), "Le nuove stime di aggregati socioeconomiche per $i$ sistemi locali del lavoro", paper presentato alla Sesta conferenza nazionale di Statistica, Roma, 6-8 Novembre 2002.

Fingleton, B. (2004), "Employment Growth of Small High-technology Firms and the Role of Horizontal Clustering: Evidence from Computing Services and R\&D in Great Britain, 1991-2000", Urban Studies vol.41 no 4, p.773-779.

Flagestad A., Hope C.A. (2001), "Strategic success in winter sports destinations: a sustainable value creation perspective" in Tourism Management, vol. 22, pp. 455-461.

Foster, N., Liu, Z., Jones, E. e Goodall, B. (1999), "Representing Wales: congruence and dissonance in tourist imagery - a systems approach". Paper presented at the RGS/IBG Symposium on 'British Tourism: the geographical research frontier', University of Exeter, Exeter. 


\section{B ibliografia}

Franch M., (2002), "Destination management : governare il turismo tra locale e globale", Giappichelli, Torino.

Frost Kumpf, H.A. (1998) Cultural district: the arts as a strategy for revitalizing our cities. Institute for Community Development and the Arts, Americans for the Arts, Washington, DC.

Garreau, J. (1992) Edge city. Anchor Books, New York.

Forni M. e Paba S., (2002) "Knowledge Spillovers and the Growth of Local Industries", Journal of Industrial Economics, 151-71.

Forni M. e Paba S., (2000) "The Sources of Local Growth: Evidence from Italy," Giornale degli Economisti e Annali di Economia, 59, 1-49.

Getz, D., (1993), "Planning for tourism business districts. Annals of Tourism Research, 20(3), 583-600.

Giannone M., (2002), "I sistemi turistici locali: un approccio alla dimesione territoriale della sviluppo turistico", in AA.VV., "Undicesimo Rapporto sul Turismo", Mercury, Firenze, pp. 601-609.

Glaeser E., Kallal H., Scheikman J.and A. Shleifer (1992), "Growth in Cities", Journal of Political Economy, no 100, p.1126-1152.

Goodall, B. e Ashworth, G. (eds) (1988), 'Marketing in the Tourism Industry: The Promotion of Destination Regions', ITBP: UK.

Gordon I., Goodall B., (2000), "Localities and Tourism" in Tourism Geographies, 2(3), 2000, 290-311.

Gordon, I.R., (1998), "Unstable jobs, unstable people and unstable places: the case of resort labour markets". Paper presented to an IBG/BSRSA conference on "The Geography of Labour Markets', Birkbeck College, London.

Gordon, I.R., (1994), "Crowding, competition and externalities in tourism development: a model of resort life cycles" in Geographical Systems 1: 289-308.

Grilinches, Z., (1979), "Issue in assessing the contribution of research and development to productivioty growth" in Bell Journal of Economics nr. 10, pp. 92-116.

Henderson V., Kunkoro e M. Turner (1995), "Industrial development in cities", The Journal of Political Economy, vol.103:5, p.1067-1090.

Henderson, J. V. (2003), "Marhsall's scale economies", in Journal of Urban Economics nr. 53, pp. 1-29.

Hoover E. M. (1937), "Location theory and the shoe and leather industries", Harvard University Press.

ISTAT (1991), "ATECO 91 - Classificazione delle attività economiche”, ISTAT, Roma.

ISTAT (2002), “ATECO 2002 - Classificazione delle attività economiche”, ISTAT, Roma.

Jacobs J. (1969), “The economy of cities”, Jonhatan Cape. London.

Jansen-Verbeke, M. C. (1986), "Contribution a` l'Analyse de la Fonction Touristique des Villes Moyennes aux Pays-Bas" in Hommes et Terres du Nord 1:21-30.

Jansen-Verbeke, M. C. e Ashworth, G., (1990), "Environmental integration of recreation and tourism" in Annals of Tourism Research, 1714), 618-622.

Judd, D. R., (1993), "Promoting tourism in US cities", Tourism Management, 16(3), 175-187.

Kim, S. (1995), "Expansion of Markets and the Geographic Distribution of Economic Activities: The Trends in U.S. Regional Manufacturing Structure, 1860-1987." Quarterly Journal of Economics 110(4): 881-908.

Kim, S. (1999), "Regions, resources and economics geography: Sources of U.S. regional 


\section{B ibliografia}

comparative advantage, 1880-1987, Regional Science and Urban Economics, 29: 1-32.

Krugman, P. (1991), "Geography and Trade", Cambridge Mass. MIT Press.

Laws, E., (1995), “Tourist Destionation Management”, Napier University, Edimburgh.

Leiper, N., (1995), “Tourism Management”, RMIT Press: Australia, PB.

Lazzeretti, L. (2004), (eds.): “Art cities, Cultural Districts and Museums", Florence University Press, Florence

Lazzeretti, L. (2003), "City of art as a HCLocal System and cultural districtualisation processes. The Cluster of art-restoration in Florence", in International Journal of Urban and Regional Research, vol. 27.3, September: 635-48.

Lazzeretti L. (2001), "I processi di distrettualizzazione culturale della città d'arte: il cluster del restauro artistico a Firenze", in Sviluppo Locale, Vol. VIII, nr. 18, Rosemberg \& Sellier, Torino.

Lazzeretti L., (1995) "Per una teorizzazione del modello Firenze", in Becheri E., (a cura di) "Il turismo a Firenze. Un modello per le città d'arte", Mercury, Firenze, pp. 399-411.

Lazzeretti L., Capone F., (2004), "Networking in the Tourist local system" in Petrillo, C. S. e Swarbrooke J. (eds.) "Networking and partnership in destination development and management", 4-6 Aprile 2004, EnzoAlbano Editore, Napoli (in corso di pubblicazione).

Lazzeretti L., Capone F. (2002), "L'evoluzione dell'offerta alberghiera di Siviglia, un'analisi demografica dal 1945 al 1999" su Turistica nr.1, 2002, paragrafi: 1.3, 2.2 2. 2.3, 3.2, 3.3, 4.

Lazzeretti L., Capone F., Brusoni G. (2002), "Dynamic and localized density dependence of organizational population foundings: a benchmarking between the case of Florence (1937-1997) and Seville (1945-1999) Hotel industry" proceedings in IXth Meeting APDR, 27th $-29^{\text {th }}$ June, 2002, Lisbona, Portogallo.

Le Sage, J, (1999), "Econometric Toolbox", (http://www.spatial-econometrics.com)

Le Sage, J.P. (1999), "The theory and practice of spatial econometrics", University of Toledo.

Leiper, N. (1995), “Tourism Management”, RMIT Press: Australia, PB.

Lomi, A. (2000), "Density dependence and spatial duality in organizational foundigs rates of Danish commercial banks 1976-1989”, American Journal of Sociology, 100, 781-813.

Manente M., Cerato M., (1999), (a cura di), "From Destination to Destination Marketing and Management", CISET, Venezia.

Martini, U., (2002) "Il Destination Management nel turismo alpino. Un quadro di riferimento concettuale e l'analisi di un caso", in Economia e diritto e diritto del terziario, Fascicolo 1, 2002.

Lòpez Palomeque, F. (1995), "La Estrategia del Turismo Metropolitano: El Caso de Barcelona" in Estudios Turisticos 126:119-141.

Marchena Gomez, M. (1995), "El Turismo Metropolitano: Una Aproximacio'n Conceptual" in Estudios Turisticos 126:7-21.

Mashall, A., (1920), "Principles of Economics", London, Man Millan.

Martin, R.L., Sunley, P., (2003), "Deconstructing Clusters: Chaotic Concept or Policy Panacea?", Journal of Economic Geography, 3, 1, 5-35.

McCann P., Sheppard S., (2003), "The rise and the fall and rise again of industrial location theory", Regional Studies, vol. 37.6\&7, pp. 649-663.

McMillen, D. (2003), "Spatial autocorrelation or model missespecification?", Internacional Regional Science Review, 26,2, 208-217.

Menghinello (a cura di) (2002), "Le esportazioni dai sistemi locali del lavoro. Dimensione locale e 


\section{B ibliografia}

competitività dell'Italia sui mercati internazionali", Roma, ISTAT, 2002.

Molteni, M. e Sainaghi, R. (1997), "Il metemanagement di un distretto turistico", in Economia e diritto del terziario, 2, 2000.

Moreno, R. and E.Vayá (2000), "Técnicas econométricas para el tratamiento de datos espacial: La econometria espacial', Edicions Universitat de Barcelona.

Mossetto G., (1992), “L'economia delle città d'arti: Modelli di sviluppo a confronto, politiche e strumenti di intervento", Milano, Etas libri.

Moulaert F. e Sekia F., (2003), “Territorial Innovation Models: A Critical Survey”, Regional Studies, 37,3, 289-302.

Nachum L., Keeble D, (2001), "External Networks and geographic clusterifn as source of MNE advantages: foreign and indigenous professional service firms in central London", Working Paper ESRC, University of Cambridge.

Nachum L., Keeble D, (2001), "Why do business service firms cluster? Small consultancies, clustering and decentralisation in london and southern england", Working Paper ESRC, University of Cambridge.

NEDLAC (1999), "South Africa's Tourism Cluster", ITC, Harvard Business School, Harvard.

Nordin, S., (2003), "Tourism clustering and innovations", no. 2003:14, ETOUR, Ostersund, Sweden.

OECD, (1999), "Territorial Indicators of Emplyment", OECD, Geneva.

Ohlin B. (1933), "Interregional and International Trade", Cambridge. Massachusetts.

Otto J. E., Ritchie B. J. R. (1996), "The service experience in tourism" in Tourism Management, vol. 17, No. 3. pp. 165-174.

Pearce D. G. (1998), "Tourist districts in Paris: structure and functions", Tourism Management, vol. 19, No. 1, pp. 49-65.

Pearce D. G. (2001), "An integrative framework for urban tourism research", Annals of Tourism Research, vol. 28, No. 4, pp. 926-946.

Pechlaner H., Weiermair K., (a cura di), (2000), "Destination Management: fondamenti di marketing e gestione delle destinazioni turistiche", Milano, TCI.

Pelizzoni, C., Turni D. (2000), "La rete come fattore critico di successo per lo sviluppo turistico di un territorio.", in Turistica, 2, 2000, Mercury, Firenze.

Petrucci, A. (2003), Dispense del corso “Analisi quantitative dei dati Territoriali”, Università di Firenze, Dipartimento di Statistica.

Petrucci, A., Romei, P. (2003), “Statistica territoriale”, Lupetti, Firenze.

Peyrache-Gadeau V. (2003), "Le developpement durable des territoires de montagne: un objet de concertation sur le devenir des stations touristiques?", paper presented at Meeting Concentration et Segregation dynamiques et inscriptions territoriales, Lyon, 1, 2 e 3 Settembre 2003.

Pilotti, L. (1997), "La reingegnerizzazione dei sistemi locali territoriali di servizio: il caso dell'area turistica commerciale gardesana" in Commercio, 60, 1997.

Porter M. E. (1990), "The Competitive advantage of nations", London, Mac Millan.

Porter M. E., (1998a), “On Competition”, Harvard Business School Press.

Porter M. E., (1998b), "Cluster and the new economics of competition”, Harvard Business Review, November-Dicember.

Porter M.E. (2003), “Regional Cluster”, Regional Studies, 2003 nr.1, pp 3-31. 


\section{B ibliografia}

Pred, A. (1977), "City systems in advanced economiees", Hutchinson, London.

Preite, M. (1998) (a cura di) La valorizzazione del patrimonio culturale in Toscana: una valutazione di alcune esperienze. Fondazione Michelucci, mimeo.

Pyke, F., Becattini, G. and Sengenberger, W. (1990), "Industrial Districts and Inter-firm Cooperation in Italy", Geneva, IILS (International Institute for Labour Studies).

Reiseinger M. E., (2003), "Secctoral Shift and Occupational Migration in the Us", The Professional Geographer, 55(3), 383-395.

Rispoli M., Tamma, M. (1995), "Risposte strategiche alla complessità: Le forme di offerta dei prodotti alberghieri”, Cedam, Padova.

Rispoli M., Tamma, M. (1996), "Le imprese alberghiere nell'industria dei viaggi e del turismo", Giappichelli, editore, Torino.

Rispoli M., e Tamma M., (1996), "Prodotto e forma di offerta: due concetti utili per interpretare i 'sistemi complessi di produzione-erogazione" in Sinergie, vol. 14, fasc. 40, p. 13-36.

Romer, P. (1986), "Increasing returns and long run growth", Journal of Political Economy, 98, 71-102.

Romer, P. (1990), "Endegenous Technological Change”, Cambridge, University Press.

Rosenfeld S. A., (1997), "Bringing Business Cluster into the mainstream of Economy development", European Planning Studies 5(1):3-23.

Rosenthal, S., Strange, W., (2003), "Evidence on the nature and sources af agglomeration Economies" in "Hanbook of Urban and Regional Economics", vol. 4.

Sainaghi, R., e De Carlo, M., (2003), (a cura di) “ Destination management e grandi eventi sportivi" in Economia \& management, vol. 6, p. 23-25.

Santagata, W., (2000), "Distretti culturali, diritti di proprietà e crescita economica sostenibile", Rassegna economica, n. 1, pp. 31-61.

Santarelli, E. (1995), "Sopravvivenza e crescita delle nuove imprese nei distretti industriali. Il settore turistico del medio adriatico", in L'industria, vol 16, 2, 1995, pp.349-362.

Santos, B. A. (2002), "Actividades emergentes - o casa do cluster do turismo / lazer", III Congreso de Tràs-os-Montes e Alto Douro, Settembre 2002.

Scott, A.J., (1996), "The craft, fashion, and cultural-product industries of Los Angeles: competitive dynamic and policy dilemma in a multisectoral image-producing complex", Annals of the Association of American Geographers, nr. 86, 306-23.

Scott, A. J. (1988), "New industrial spaces: flexible production organisation and regional development in North America and Western Europe", London, Pion.

Sforzi F. (1985), "Riflessioni sul distretto industriale: un'ipotesi di identificazione spaziale", in R. Innocenti (a cura di), "Piccola città \& piccola Impresa", Milano, Franco Angeli, pp. 247-267.

Sforzi F. (a cura di), (1997a), "I sistemi locali in italia", ISTAT.

Sforzi F., (1997b), "Il cambiamento economico nel sistema urbano italiano" in Dematteis G. e Bonavero P. (a cura di) "Il Sistema urbano italiano nello spazio unificato europeo", Il Mulino, Bologna.

Sforzi, F. (2002), "The Industrial district and the 'new' Italian economic geography" European Planning Studies, 2002(4).

Solinas, G. e Baroni D., "I sistemi locali manifatturieri in Italia: 1991- 1996", in G. Becattini e altri (a cura di), Il caleidoscopio dello sviluppo locale. Trasformazioni economiche 


\section{B ibliografia}

nell'Italia contemporanea, Torino, Rosenberg \& Sellier, 2001, pp. 395-417.

Stansfield, C. e Rickert, J., (1970), “The recreational business district” in Journal of Leisure Research, 2(4), 238-251.

Suzigan W., Furtado J., Garcia R. e Sampaio S, (2004), "Local production systems: mapping, typology and policy suggestions" paper presentato al European Regional Science Association (ERSA) 2004 Congress, University of Porto, Porto, Portugal, 25-29 August 2004.

Tamma, M., (1999), "Destination management e logica di sistema" in La Rivista del Turismo, vol. 1, vol. 2, p. 46-48.

Trigilia C. (1988), "Grandi partiti e piccole imprese. Comunisti e democristiani nelle regioni a economia diffusa”, Bologna, Il Mulino.

Trullén, J., (2002), "La economía de Barcelona y la generación de economías de aglomeración: hacia un nuevo modelo de desarrollo" in Becattini, Costa y Trullén (eds) "Desarrollo local, teorías y estrategias", Ed. Civitas, Madrid.

Trullén, J., (2003), "Redes de ciudades y economía del conocimiento. La CataluñaCiudades" in Antonio Font (a cura di) "Planeamiento Urbanistico: de la controversia a la renovación" Diputació de Barcelona, p.49-65.

Trullén, J., e Boix R. (2001), "Economia della conoscenza e reti di città" in Sviluppo Locale, Vol. VIII, n. 18, pp. 41-60.

Trullén, J. e Boix R. (2003), "Barcelona, policentric metropolis and network of cities" presentato al Workshop on Spatial Networks and Clusters, Universitat Rovira i Virgili, Marzo, 2003.

Trullén, J., Boix R. (2004), "Knowledge, networks of cities and growth in regional urban systems: theory, measurement and policy implications", paper presentato al European Regional Science Association (ERSA) 2004 Congress, University of Porto, Porto, Portugal, 2529 August 2004.

Trullén, J.; Lladòs, J. y Rafael Boix (2002), "Economía del conocimiento, ciudad y competitividad", in Investigaciones Regionales, $\mathrm{n}^{\circ}$ 1, p. 139-161.

TCI, Competitiveness Institute, (1998), “Tourism Cluster in Lloret de Mar", Barcellona.

TCI, Competitiveness Institute, (1998), “Tourism Industry cluster in Bilbao", Barcellona.

TCI, Competitiveness Institute, (1998), "Tourism Industtry Cluster in San Sebastian", Barcellona.

Valentino P. A. (2001), I distretti culturali. Nuove opportunità di sviluppo del territorio, Roma, Associazione Civita.

Van Den Berg L., Braum, E. e Van Winden W., (2001), "Growth cluster in European Metropolitan cities", Aldershot, Burlinghton, AshGate.

Van den Berg L., Van der Borg, J. e Van der Meer, J., (1995), "Urban Tourism PerJormance and Strategies in Eight European Cities. Avebury, Aldershot, 1995

Weber A. (1929): "Theory of the location of industries", University of Chicago Press. Chicago (ed.orig.ted.1909).

Zanni L., (1995),"Imprenditorialità e Territorio" ,Cedam, Padova. 


\section{INDICE DELLE FIGURE}

Figura 1.1: Il Sistema Locale Turistico (SLT): il cluster di attori e la comunità sociale.

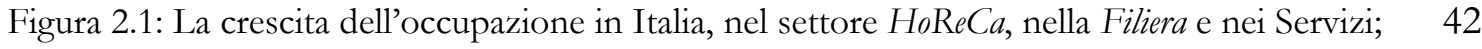

Figura 2.2: Mappa dei sistemi locali dei servizi al consumatore, 1991;

Figura 2.3: Distretti turistici secondo l'ISTAT;

Figura 2.4: I sistemi economici locali turistici nella regione Toscana; 45

Figura 2.5: Mappa dei distretti turistici; 46

Figura 2.6: Il "settore" HoReCa in Italia;

Figura 2.7: A tourism sector model;

Figura 2.8: I sistemi locali del lavoro in base alla filiera turistica della tabella 2.4; 61

Figura 2.9: Sistemi Locali Turistici per classi di specializzazione; $\quad 62$

Figura 2.10: Sistemi Locali del lavoro settore HoReCa (Voce H) su dati 2001; 63

Figura 2.11: La crescita dell'occupazione della filiera turistica nei SLL 1991-2001; 65

Figura 2.12: Arrivi totali (quartili), Italia 2001; 66

$\begin{array}{ll}\text { Figura 2.13: Arrivi totali ponderati sulla filiera; } & 67\end{array}$

Figura 2.14: Spesa relativa dei turisti in Italia in base ai lavoratori nella filiera; $\quad 68$

Figura 2.15: Sistemi Locali del lavoro in base alla K-means Cluster; $\quad 71$

Figura 3.1: La classificazione delle economie esterne e spillovers; 86

Figura 4.1: Esempi di Spatial dependence; $\quad 87$

Figura 4.2: Propagazione delle Falene nello stato del Michigan. 88

Figura 4.3: Scatterplot di Moran (univariate). 95

Figura 4.4: ScatterMap e scatterplot della criminalità in Columbus; 96

Figura 5.1: Il PACA nei SLL in Italia; $\quad 112$

Figura 5.2: La crescita dell'occupazione della filiera turistica nei SLL, 1991-2001; 113

$\begin{array}{ll}\text { Figura 5.3: I di Moran; } & 115\end{array}$

$\begin{array}{ll}\text { Figura 5.4: le reti di SLL e cluster map; } & 116\end{array}$ 


\section{INDICE DELLE TABELLE}

Tabella: 1.1: Stato attuale del riconoscimento dei STL in base alla 135/2001.

Tabella 1.2: I modelli sistemici applicati al turismo: caratteristiche principali.

Tabella 2.1: I distretti del rapporto ACI-CENSIS.

Tabella 2.2: Variabili economiche utilizzate e dettaglio territoriale di riferimento; $\quad 48$

Tabella 2.3: Voce H. Hotel e Ristoranti - Codice Ateco; 51

Tabella 2.4: Attività collegate al turismo :Appendice E - ATECO 91

Tabella 2.5: SLL con indici di concentrazione più alti 64

Tabella 2.6: K-Means Cluster Analysis $\quad 70$

Tabella 2.7: Tipologia ed esempi di SLT in Italia; $\quad 72$

Tabella 4.1: Patterns spaziali e Matrice dei contatti; 90

Tabella 4.2: Patterns spaziali e Matrice dei contatti; $\quad 90$

Tabella 4.3: Tests univariati per la dipendenza spaziale; $\quad 90$

Tabella 4.4: Tests univariati per la dipendenza spaziale; $\quad 90$

Tabella 4.5: I Softwares per la econometria spaziale; $\quad 91$

Tabella 4.6: Principali applicazioni empiriche della Econometria spaziale, 1990-1999; 92

Tabella 4.7: Indici locali di dipendenza spaziale; 96

Tabella 4.8: Analisi con il software SpaceStat: metodi di regressione e test di diagnostica; $\quad 99$

Tabella 5.1: Variabili del modello. 105

Tabella 5.2: Regressioni per gli effetti di localizzazione, urbanizzazione relazioni input-utput; 107

Tabella 5.3: Risultati dei modelli spaziali: unità d'analisi comunale; 108

Tabella 5.4: Regimi spaziali; 109

Tabella 5.5: Variabili del modello.

Tabella 5.6: Regressioni per gli effetti di localizzazione, urbanizzazione relazioni input-utput; 117

Tabella 5.7: Risultati modelli spaziali; 118

Tabella 5.8: Regimi spaziali; 119 


\section{APPENDICI}

APPENDICE 1.1: I SISTEMI LOCALI DEL LAVORO DEL 1991

APPENDICE 2.1: VOCE H ALBERGHI E RISTORANTI

APPENDICE 2.2: FILIERA ALLARGATA;

APPENDICE 2.4: SLT - SPESE ITALIANI ALL'ESTERO E PRESENZE;.

APPENDICE 5.1: L'INDICE INVERSO HERFINDHAL-HIRSCHMAN (IHHI)... 145

\section{APPENDICE 1.1: I SISTEMI LOCALI DEL LAVORO DEL 1991}

Abbadia San Salvatore, Accadia, Acerenza, Acireale, Acquapendente, Acqui Terme, Acri, Adrano, Adria, Agerola, Agnone, Agordo, Agrigento, Agropoli, Airola, Alassio, Alba, Albenga, Albino, Alcamo, Alcara Li Fusi, Ales, Alessandria, Alghero, Amalfi, Amantea, Amatrice, Ancona, Aosta, Apice, Aprilia, Aradeo, Arezzo, Argenta, Ariano Irpino, Arzachena Arzignano, Ascea, Ascoli Piceno, Ascoli Satriano, Asiago, Assisi, Asti, Atri, Aulla, Avellino, Aversa, Avezzano, Avigliana, Badia, Badia Polesine, Badolato, Bagheria, Bagno Di Romagna, Bagnoregio, Barcellona Pozzo Di Gotto, Bardonecchia, Barga, Barge, Bari, Barletta, Bassano Del Grappa, Battipaglia, Bedonia, Bella, Bellagio, Belluno, Belvedere Marittimo, Bene Vagienna Benetutti, Benevento, Bergamo, Bianco, Bibbiena, Biccari, Biella, Bisceglie, Bisignano, Bitti, Bivona, Bleggio Inferiore, Bobbio, Bologna, Bolzano, Bono, Bonorva, Borgo Val Di Taro, Borgo Valsugana, Borgomanero, Borgosesia, Bormio, Bosa, Botricello, Bovalino, Bovino, Bovolone, Brescia, Bressanone, Brindisi, Brolo, Bronte, Brunico, Buccino, Budduso', Budoni, Busseto, Busto Arsizio, Cagli, Cagliari, Cairo Montenotte, Calitri, Caltagirone, Caltanissetta, Caltavuturo, Camerino, Camerota, Cammarata, Campione D'italia, Campo Nell'elba, Campo Tures, Campobasso, Campobello Di Licata, Canazei, Candela, Canelli, Cannobio, Capaccio, Capizzi, Capo D'orlando, Capri, Cariati, Carmagnola, Carpi, Carrara, Carru', Casacalenda, Casale Monferrato, Casalmaggiore, Casalnuovo Monterotaro, Casarano, Cascia, Caserta, Casoli, Cassano Allo Ionio, Cassino, Castagnaro, Castel Del Piano, Castel Di Sangro, Castel Goffredo, Castel San Giovanni, Castel San Lorenzo, Castelfiorentino, Castelforte, Castelfranco Veneto, Castellabate, Castellammare Di Stabia, Castellarano, Castelleone, Castelmassa, Castelnovo $\mathrm{Ne}^{\prime}$ Monti, Castelnuovo Di Garfagnana, Castelrotto, Castelsardo, Casteltermini, Castelvetrano, Castiglione Del Lago, Castiglione Delle Stiviere, Castilenti, Castronuovo Di Sicilia, Castrovillari, Catania, Catanzaro, Cattolica, Cavalese, Cavarzere, Cecina, Cefalu', Celano, Cento, Cerchiara Di Calabria, Cerea, Cerignola, Cerreto Sannita, Cerrina Monferrato, Cesena, Cetraro, Ceva, Chiaravalle Centrale, Chiari, Chiavari, Chiavenna, Chieri, Chiesa In Valmalenco, Chieti, Chiusi, Cingoli, Circello, Cirie', Ciro' Marina, Citta' Di Castello, Cittadella, Civita Castellana, Civitanova Marche, Civitavecchia, Cles, Clusone, Codigoro, Collesano, Comacchio, Como, Comunanza, Condofuri, Conegliano, Copparo, Cori, Corigliano Calabro, Corleone, Correggio, Cortemilia, Cortina D'ampezzo, Cortona, Cosenza, Cossato, Cotronei, Crecchio, Crema, Cremona, Crescentino, Crodo, Crosia, Crotone, Cuneo, Custonaci, Darfo Boario Terme, Delianuova, Desenzano Del Garda, Desio, Diamante, Diano Marina, Dinami, Dogliani, Domodossola, Eboli, Edolo, Egna, Empoli, Enna, Este, Fabriano, Fabrizia, Fabro, Faenza, Fagnano Castello, Fanano, Fano, Feltre, Fermo, Ferrara, Fiano Romano, Fidenza, Fiera Di Primiero, Finale Ligure, Fiorenzuola D'arda, Firenze, Fiuggi, Foggia, Foligno, Follonica, Fondi, Fondo, Fonni, Forio, Forli', Formia, Fornovo Di Taro, Fossano, Fossombrone, Francavilla Di Sicilia, Francavilla In Sinni, Frasso Telesino, Frosinone, Frosolone, Gaiole In Chianti, Gallarate, Gallipoli, Gambara, Gangi, Gattinara, Gavi, Gela, Genova, Ghilarza, Giarre, Ginosa, Gioia Del Colle, Gioiosa Ionica, Girifalco, Giulianova, Gorizia, Grammichele, Gravina In Puglia, Grosseto, Gualdo Tadino, Guardavalle, Guardia Sanframondi, Guardiagrele, Guastalla, Gubbio Iglesias, Imola, Imperia, Ischia, Iseo, Isernia, Isili, Isola Del Gran Sasso D'italia, Isola Della Scala, Ivrea, Jerzu, Jesi, La Maddalena, La Spezia, Lamezia Terme, Lanciano, Langhirano, Lanusei, L'aquila, Latina, Latisana, 
Latronico, Lauria, Laurino, Lecce, Lecco, Legnago, Lentini Lercara Friddi, Lesina, Levico Terme, Limone Sul Garda, Lioni, Lipari, Livorno, Locri, Lodi, Lonigo, Lovere, Lucca, Lucera, Lugo, Luino, Lumezzane, Lungro, Macerata, Macomer, Maglie, Maierato, Maiori, Malcesine, Male', Malles Venosta, Manciano, Mandatoriccio, Manerbio, Manfredonia, Maniago, Manoppello, Mantova, Marcaria, Marina Di Gioiosa Ionica, Marostica, Marradi, Marsala, Marsciano, Marsicovetere, Martina Franca, Masone, Massa, Matera, Mazzarino, Mede, Melfi, Melito Di Porto Salvo, Menaggio, Merano, Mercato Saraceno Messina, Mezzolombardo, Milano, Milazzo, Mirandola, Mistretta, Modena, Modica, Modigliana, Moena, Mogoro, Moliterno, Mondolfo, Mondovi', Monfalcone, Monselice, Montagnana, Montalbano Elicona, Montalcino, Montano Antilia, Monte San Giusto, Monte San Pietrangeli, Montebello Ionico, Montebelluna, Montecalvo Irpino, Montecatini-Terme, Montefiore Dell'aso, Montegiorgio, Montegranaro, Montella, Montemiletto, Montepulciano, Montesarchio, Montese, Montichiari, Morbegno, Morciano Di Romagna, Morcone, Moretta, Morgex, Mormanno, Mortara, Muravera, Muro Lucano, Mussomeli, Napoli, Nardo', Naro, Naturno, Nicosia, Nicotera, Nizza Monferrato, Nocera Inferiore, Nocera Tirinese, Nola, Norcia, Noto,, Nova Ponente, Novafeltria, Novara, Novi Ligure, Nuoro, Oderzo, Offida, Olbia, Oleggio, Oliveto Citra, Omegna, Oppido Mamertina, Orbetello, Oristano, Orosei, Orsogna, Orta Nova, Orte, Ortisei, Orvieto, Orzinuovi, Oschiri, Osimo, Ostiglia, Ostra, Ovada, Ovaro, Ozieri, Pachino, Padova, Palagonia, Palazzolo Acreide, Palazzolo Sull'oglio, Palermo, Palestrina, Palmi, Palomonte, Paola, Parma, Partanna, Partinico, Patti, Pavia, Pavullo Nel Frignano, Peio, Pennabilli, Penne, Perano, Perfugas, Pergola, Perugia, Pesaro, Pescara, Pescopagano, Petilia Policastro, Petralia Sottana, Piacenza, Piana Degli Albanesi, Piandimeleto, Piazza Armerina, Piedimonte Matese, Pietramontecorvino, Pietrasanta, Pietrelcina Pieve D'alpago, Pieve Di Cadore, Pieve Di Soligo, Pieve Di Teco, Pieve Santo Stefano, Pievepelago, Pinerolo, Pinzolo, Piombino, Pisa, Pisticci, Pistoia, Pitigliano, Pizzo, Poggiardo, Poggibonsi, Policoro, Polizzi Generosa, Pollica, Pomarance, Ponte Di Legno, Pontebba, Pontedera, Pontevico, Pontremoli, Pont-Saint-Martin, Popoli, Pordenone, Porlezza, Porretta Terme, Porto Azzurro, Porto Sant'elpidio, Porto Tolle, Portoferraio, Portogruaro, Positano, Postiglione, Potenza, Pozzomaggiore, Praia A Mare, Prato, Pratovecchio, Predazzo, Premana, Priverno, Prizzi, Pula, Putignano, Quinzano D'oglio, Racalmuto, Ragusa, Randazzo, Rapallo, Ravenna, Recanati, Regalbuto, Reggio Di Calabria, Reggio Nell'emilia, Ribera, Riccia, Rieti, Rimini, Rionero In Vulture, Riva Del Garda, Rivarolo Canavese, Rocca Imperiale, Rocca San Casciano, Roccastrada, Roccella Ionica, Rogliano, Roma, Romano Di Lombardia, Rombiolo, Rosarno, Rosignano Marittimo, Rossano, Rotonda, Rotondella, Rovereto, Rovigo, SaintVincent, Sala Consilina, Salandra, Salemi, Salerno, Salo', Saluzzo, Samugheo, San Bartolomeo In Galdo, San Benedetto Dei Marsi, San Benedetto Del Tronto, San Bonifacio, San Candido, San Dona' Di Piave, San Fratello, San Giorgio Lucano, San Giovanni Ilarione, San Giovanni Rotondo, San Giuseppe Jato, San Giuseppe Vesuviano, San Lucido, San Marcello Pistoiese, San Marco Argentano, San Marco Dei Cavoti, San Martino In Passiria, San Piero Patti, San Pietro Vernotico, San Quirico D'orcia,, San Remo, San Severo, Sanluri, Sannicandro Garganico, Sansepolcro, Santa Croce Di Magliano, Santa Croce Sull'arno, Santa Margherita Di Belice, Santa Maria Maggiore, Santa Sofia, Santa Teresa Di Gallura, Santa Teresa Di Riva, Sant'agata De' Goti, Sant'agata Di Esaro, Sant'agata Di Militello, Sant'ambrogio Di Valpolicella, Sant'angelo Dei Lombardi, Sant'angelo In Vado, Sant'angelo Le Fratte, Sant'angelo Lodigiano, Sant'antioco, Sant'arcangelo, Sant'eufemia D'aspromonte, Santo Stefano Di Cadore, Santo Stefano Di Camastra Sapri, Sarnano, Sarno, Sassari, Sassocorvaro, Sassoferrato, Sassuolo, Savona, Scalea, Schio, Sciacca, Scordia, Senigallia, Senise, Senorbi', Sermide, Serra De'conti, Serra San Bruno, Serracapriola, Sersale, Sessa Aurunca, Sesto Calende, Sestri Levante, Siena, Silandro, Silius, Sinalunga, Siniscola, Siracusa, Solofra, Sondalo, Sondrio, Sora,, Soresina, Sorgono, Soriano Calabro, Sorrento, Soverato, Soveria Mannelli, Spezzano Albanese, Spezzano Della Sila, Spilimbergo, Spinazzola, Spoleto, Stigliano, Stilo, Storo, Stradella, Strongoli, Sulmona, Suzzara Taormina, Taranto, Tarquinia, Tarvisio, Taurasi, Taurianova, Taverna, Teano, Telese, Tempio Pausania, Teramo, Termini Imerese, Termoli, Terni, Terracina, Terralba, Terrasini, Thiene, Thiesi, Tione Di Trento, Tirano, Tizzano Val Parma, Todi, Tolentino, Tolmezzo, Torano Castello, Torino, Torre Annunziata, Torrecuso, Tortoli', Tortona, Tortorici, ToscolanoMaderno, Trapani, Trecenta, Treia, Trento, Treviglio, Treviso, Tricarico, Tricase, Trieste, Trivento, Troina, Tropea, Tuscania, Udine, Umbertide, Urbania, Urbino, Urbisaglia, Valdagno, Valentano, Vallata, Valledoria, Vallo Della Lucania, Varallo, Varese, Varzi, Vasto, Velletri, Venafro, Venezia, Venosa, Ventimiglia, Verbania, Vercelli, Verona, Verzino, Verzuolo, Vestone, Viadana, Viareggio, 
Vibo Valentia, Vicenza, Vico Del Gargano, Vieste, Vigevano, Vignola, Villa Santa Maria, Villacidro, Villafranca Tirrena, Villar Perosa, Vilminore Di Scalve, Vipiteno, Visso, Viterbo, Vittoria, Vittorio Veneto, Vizzini, Voghera, Volterra, Zogno.

\section{APPENDICE 2.1: VOCE H ALBERGHI E RISTORANTI}

In questa sezione sono incluse le unità che forniscono ai clienti alloggio e/o che preparano pasti, spuntini e bevande pronti per il consumo. Sono inclusi tanto i servizi di alloggio quanto i servizi di ristorazione in quanto entrambe le attività sono spesso svolte dalla stessa unità.

Alcune attività della sezione si sovrappongono in quanto la somministrazione di bevande è un'attività indipendente che fa tuttavia parte dell'attività di ristorazione (fornitura di pasti con bevande); in quest'ultimo caso la somministrazione di bevande è classificata insieme alla somministrazione di pasti in 55.3 (Ristoranti).

Allo stesso modo l'attività di ristorazione è un'attività specifica che può tuttavia essere implicitamente inclusa nella fornitura di alloggio. Le unità di alloggio forniscono servizi, anche di breve durata, a viaggiatori, turisti e altri. Esiste una vasta gamma di unità: alcune forniscono unicamente alloggio, mentre altre forniscono anche pasti e attività ricreative.

Il tipo di servizi accessori forniti varia da un'unità all'altra. Le unità che appartengono al gruppo dei ristoranti forniscono pasti completi adatti al consumo immediato. Possono essere ristoranti tradizionali, self-service, ristoranti che preparano cibo da asporto, banchi permanenti o temporanei per la vendita di pesce e patatine fritte, o simili con o senza posti a sedere. L'aspetto decisivo è che vengano forniti pasti per il consumo immediato, indipendentemente dal tipo di servizio offerto. È esclusa la fornitura di pasti non adatti al consumo immediato o che non si intende consumare immediatamente o di cibo preparato che non può essere considerato un pasto (cfr. 15: Industrie alimentari e delle bevande).

È esclusa altresí la vendita di cibo non fabbricato in proprio che non può essere considerato un pasto o di pasti inadatti al consumo immediato (cfr. sezione G: Commercio all'ingrosso e al dettaglio).

\subsection{Alberghi.}

55.10 Alberghi.

55.10.0 Alberghi. - fornitura di alloggio di breve durata presso: alberghi, hotel, motel e locande, pensioni, hotel attrezzati per ospitare conferenze (compresi quelli con attività mista di fornitura di alloggio e somministrazione di pasti e bevande)

Dalla classe 55.10 sono escluse: - affitto di alloggi per periodi di tempo prolungati cfr. 70.20 , attività connesse con la multiproprietà cfr. 70.20

55.2 Campeggi ed altri alloggi per brevi soggiorni. In questo gruppo vengono classificate le attività che consistono nella fornitura di alloggio per periodi di breve durata, presso: campi di villeggiatura, chalet, appartamenti, terreni e infrastrutture per campeggio, residence, alloggi presso, aziende agricole che praticano l'agriturismo, ostelli della gioventù, rifugi di montagna, ecc.

Da questo gruppo sono escluse le attività di affitto di alloggi per periodi di tempo prolungati cfr. 70.20

55.21 Ostelli della gioventù e rifugi di montagna:

55.21.1 Ostelli della gioventù:

55.21.2 Rifugi di montagna: Inclusi quelli con attività mista di fornitura di alloggio e somministrazione di pasti e bevande

55.22 Campeggi ed aree attrezzate per roulotte

55.22.0 Campeggi ed aree attrezzate per roulotte

55.23 Altri tipi di alloggio, 55.23.1 Villaggi turistici

55.23.2 Colonie marine e montane

55.23.3 Gestione di vagoni letto

55.23.4 Affittacamere per brevi soggiorni, case ed appartamenti per vacanze, bed and breakfast, residence, - fornitura di alloggio di breve durata presso: chalet, villette e appartamenti per vacanze e altre infrastrutture n.c.a 
55.23.5 Agriturismo, Inclusi quelli con attività mista di fornitura di alloggio e somministrazione di pasti e bevande

55.23.6 Alloggi per studenti e lavoratori con servizi di tipo alberghiero, - gestione di case dello studente, residenze universitarie, pensionati per studenti, pensionati per lavoratori immigrati, altre infrastrutture n.c.a, 86, Dalla classe 55.23 sono escluse:- agriturismo e rifugi di montagna con sola ristorazione, senza alloggio cfr. 55.30, - servizi di locazione di beni immobili cfr. 70

\subsection{Ristoranti}

55.30 Ristoranti

55.30.1 Ristorazione con somministrazione, - fornitura di pasti da consumare in genere sul posto, come pure la somministrazione di bevande che accompagnano tali, pasti, eventualmente con intrattenimento e spettacolo.

55.30.2 Ristorazione con preparazione di cibi da asporto, - ristorazione senza somministrazione, ma con preparazione di pasti da portar via " take-away ", - attività degli esercizi di gastronomia, rosticcerie, friggitorie, pizzerie a taglio, ecc., che si caratterizzano per la mancanza di strutture atte a consentire la consumazione di cibi e bevande ai tavoli

55.30.3 Ristorazione su treni e navi, - servizi di ristorazione su treni, traghetti, navi e altri tipi di mezzi di trasporto passeggeri

55.30.4 Gelaterie e pasticcerie con somministrazione, - pubblici esercizi di gelaterie e pasticcerie con somministrazione, Dalla classe 55.30 sono escluse: - produrione industriale e artigianale di gelati, sorbetti e altri prodotti commestibili simili (senza somministrazione) cfr. 15.52, - fabbricazione di prodotti di pasticceria (senza somministrazione) cfr. 15.81 e 15.82,- produzione di pizze a lunga conservazione cfr. 15.82 commercio attraverso distributori automatici cfr. 52.63

\subsection{Bar}

55.40 Bar

55.40.0 Bar, caffètterie, altri esercizi con somministrazione di bevande, - attività di bar, caffè, pub, birrerie, ecc., eventualmente con intrattenimento e spettacolo. Dalla classe 55.40 sono escluse:, commercio attraverso distributori automatici cfr. 52.63, - vendita di bevande per il consumo al di fuori dai locali pubblici cfr. 52,

55.5 Mense e fornitura di pasti preparati

55.51 Mense

55.51.0 Mense,- somministrazione di cibi e bevande, in genere a prezzo ridotto, a gruppi di persone chiaramente definiti aventi vincoli di natura professionale: attività delle mense aziendali, mense sportive, mense scolastiche, mense universitarie, mense e posti di ristoro per i membri delle forze armate, ecc.

55.52 Fornitura di pasti preparati (catering)

55.52.0 Fornitura di pasti preparati (catering, banqueting), - attività di imprenditori che forniscono cibi preparati presso un'unità centrale e destinati al consumo in altra sede, ad es. fornitura a compagnie aeree, per il recapito a domicilio, per banchetti, cene di rappresentanza, per matrimoni, ricevimenti ed altre celebrazioni o cerimonie (Istat, 2003).

\section{APPENDICE 2.2: FILIERA ALLARGATA;}

01.13 Coltivazione di frutta, frutta a guscio, prodotti destinati alla preparazione di bevande, spezie

01.13.1 Colture viticole

01.13.2 Colture olivicole

01.13.3 Colture agrumicole

01.13.4 Colture frutticole diverse, coltivazione di prodotti destinati alla preparazione di bevande e spezie

01.13.5 Colture miste viticole, olivicole e frutticole

26.1 FABBRICAZIONE DI VETRO E DI PRODOTTI IN VETRO [Artigiani] 26.11 Fabbricazione di vetro piano

26.11.0 Fabbricazione di vetro piano 
26.12 Lavorazione e trasformazione del vetro piano

26.12.0 Lavorazione e trasformazione del vetro piano

26.13 Fabbricazione di vetro cavo

26.13.0 Fabbricazione di vetro cavo

26.14 Fabbricazione di fibre di vetro

26.14.0 Fabbricazione di fibre di vetro

26.15 Fabbricazione e lavorazione di altro vetro (incluso vetro per usi tecnici), lavorazione di vetro cavo

26.15.1 Lavorazione e trasformazione del vetro cavo

26.15.2 Lavorazione di vetro a mano e a soffio

26.15.3 Fabbricazione e lavorazione di vetro tecnico, industriale, per altri lavori

\subsection{FABBRICAZIONE DI PRODOTTI CERAMICI NON REFRATTARI, NON DESTINATI ALL'EDILIZIA E DI PRODOTTI CERAMICI REFRATTARI [Artigiani]}

26.21 Fabbricazione di prodotti in ceramica per usi domestici e ornamentali

26.21.0 Fabbricazione di prodotti in ceramica per usi domestici e ornamentali

26.22 Fabbricazione di articoli sanitari in ceramica

26.22.0 Fabbricazione di articoli sanitari in ceramica

26.23 Fabbricazione di isolatori e di pezzi isolanti in ceramica

26.23.0 Fabbricazione di isolatori e di pezzi isolanti in ceramica

26.24 Fabbricazione di altri prodotti ceramici per uso tecnico e industriale

26.24.0 Fabbricazione di altri prodotti ceramici per uso tecnico e industriale

26.25 Fabbricazione di altri prodotti ceramici

26.25.0 Fabbricazione di altri prodotti ceramici

26.26 Fabbricazione di prodotti ceramici refrattari

\section{ALBERGHI E RISTORANTI}

55.1 ALBERGHI

55.10 Alberghi

55.10.0 Alberghi

55.2 CAMPEGGI ED ALTRI ALLOGGI PER BREVI SOGGIORNI

55.21 Ostelli della gioventù e rifugi di montagna

55.21.1 Ostelli della gioventù

55.21.2 Rifugi di montagna

55.22 Campeggi ed aree attrezzate per roulotte

55.22.0 Campeggi ed aree attrezzate per roulotte

55.23 Altri tipi di alloggio

55.23.1 Villaggi turistici

55.23.2 Colonie marine e montane

55.23.3 Gestione di vagoni letto

55.23.4 Affittacamere per brevi soggiorni, case ed appartamenti per vacanze, bed and breakfast, residence

55.23.5 Agriturismo

55.23.6 Alloggi per studenti e lavoratori con servizi di tipo alberghiero

\subsection{RISTORANTI}

55.30 Ristoranti

55.30.1 Ristorazione con somministrazione

55.30.2 Ristorazione con preparazione di cibi da asporto

55.30.3 Ristorazione su treni e navi

55.30.4 Gelaterie e pasticcerie con somministrazione

55.4 BAR

55.40 Bar

55.40.0 Bar, caffetterie, altri esercizi con somministrazione di bevande

\subsection{LOCAZIONE DI BENI IMMOBILI}


70.20 Locazione di beni immobili

70.20.0 Locazione di beni immobili

\subsection{NOLEGGIO DI AUTOVETTURE}

71.10 Noleggio di autovetture

71.10.0 Noleggio di autovetture

\subsection{ALTRE ATTIVITÀ DELLO SPETTACOLO, DI INTRATTENIMENTO E DIVERTIMENTO \\ 92.31 Creazioni e interpretazioni artistiche e letterarie \\ 92.31.0 Creazioni e interpretazioni artistiche e letterarie \\ 92.32 Gestione di teatri, sale da concerto e altre sale di spettacolo e attività connesse \\ 92.32.0 Gestione di teatri, sale da concerto e altre sale di spettacolo e attività connesse \\ 92.33 Attività dei parchi di divertimento \\ 92.33.0 Attività dei parchi di divertimento}

\subsection{ATTIVITÀ DI BIBLIOTECHE, ARCHIVI, MUSEI ED ALTRE ATTIVITÀ CULTURALI}

92.51 Attività di biblioteche e archivi

92.51.0 Attività di biblioteche e archivi

92.52 Gestione di musei e del patrimonio culturale

92.52.0 Gestione di musei e del patrimonio culturale

92.53 Gestione degli orti botanici, dei parchi naturali e del patrimonio naturale

92.53.0 Gestione degli orti botanici, dei parchi naturali e del patrimonio naturale

\subsection{ATTIVITÀ SPORTIVE}

92.61 Gestione di stadi ed altri impianti sportivi

92.61.1 Gestione di stadi

92.61.2 Gestione di piscine

92.61.3 Gestione di campi da tennis

92.61.4 Gestione di impianti polivalenti

92.61.5 Gestione di palestre sportive

92.61.6 Gestione di altri impianti sportivi nca

92.62 Altre attività sportive

92.62.1 Enti ed organizzazioni sportive, promozione di eventi sportivi

92.62.2 Attività professionali sportive indipendenti prestate da atleti professionisti

92.62.3 Attività professionali svolte da operatori sportivi indipendenti

92.62.4 Società sportive ed altri nuclei organizzativi di base

\subsection{ATTIVITÀ RICREATIVE}

92.71 Attività riguardanti le lotterie, le scommesse, le case da gioco

92.71.0 Attività riguardanti le lotterie, le scommesse, le case da gioco

92.72 Altre attività ricreative

92.72.1 Gestione di stabilimenti balneari: marittimi, lacuali e fluviali

92.72.2 Sale giochi e biliardi

92.72.3 Altre attività ricreative nca

\subsection{TRASPORTI FERROVIARI}

60.10 Trasporti ferroviari

60.10.0 Trasporti ferroviari

- trasporti ferroviari interurbani e suburbani di passeggeri

\subsection{TRASPORTI FERROVIARI}

60.10 Trasporti ferroviari

60.10.0 Trasporti ferroviari

\subsection{ALTRI TRASPORTI TERRESTRI}


60.21 Altri trasporti terrestri, regolari, di passeggeri

60.21.0 Altri trasporti terrestri, regolari, di passeggeri

60.23 Altri trasporti terrestri di passeggeri

60.23.0 Altri trasporti terrestri non regolari di passeggeri

\subsection{TRASPORTI MARITTIMI E COSTIERI}

61.10 Trasporti marittimi e costieri

61.10.0 Trasporti marittimi e costieri

\subsection{TRASPORTI PER VIE D'ACQUA INTERNE (COMPRESI I TRASPORTI LAGUNARI) \\ 61.20 Trasporti per vie d'acqua interne (compresi i trasporti lagunari) \\ 61.20.0 Trasporti per vie d'acqua interne (compresi i trasporti lagunari)}

\section{TRASPORTI AEREI}

\subsection{TRASPORTI AEREI DI LINEA}

62.10 Trasporti aerei di linea

62.10.0 Trasporti aerei di linea

63.3 ATTIVITÀ DELLE AGENZIE DI VIAGGIO E DEGLI OPERATORI TURISTICI; ATTIVITÀ

DI ASSISTENZA TURISTICA

63.30 Attività delle agenzie di viaggio e degli operatori turistici; attività di assistenza turistica

63.30.1 Attività delle agenzie di viaggio e turismo e dei tour operator

63.30.2 Attività delle guide e degli accompagnatori turistici 
APPENDICE 2.3: SLT PER SPECIALIZZAZIONE;

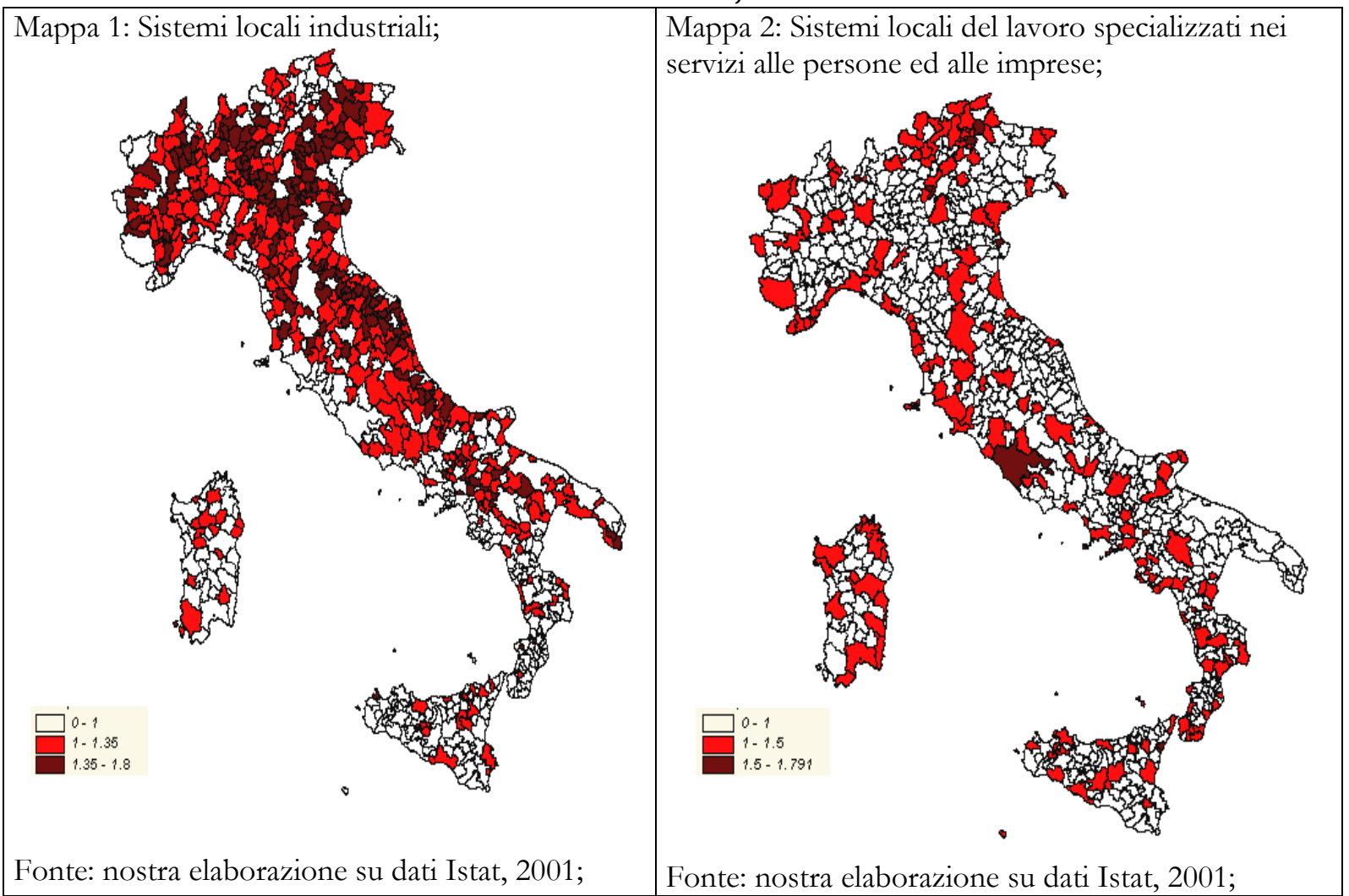

Fonte: nostra elaborazione;

APPENDICE 2.4: SLT SPESE ITALIANI ALL'ESTERO E PRESENZE;

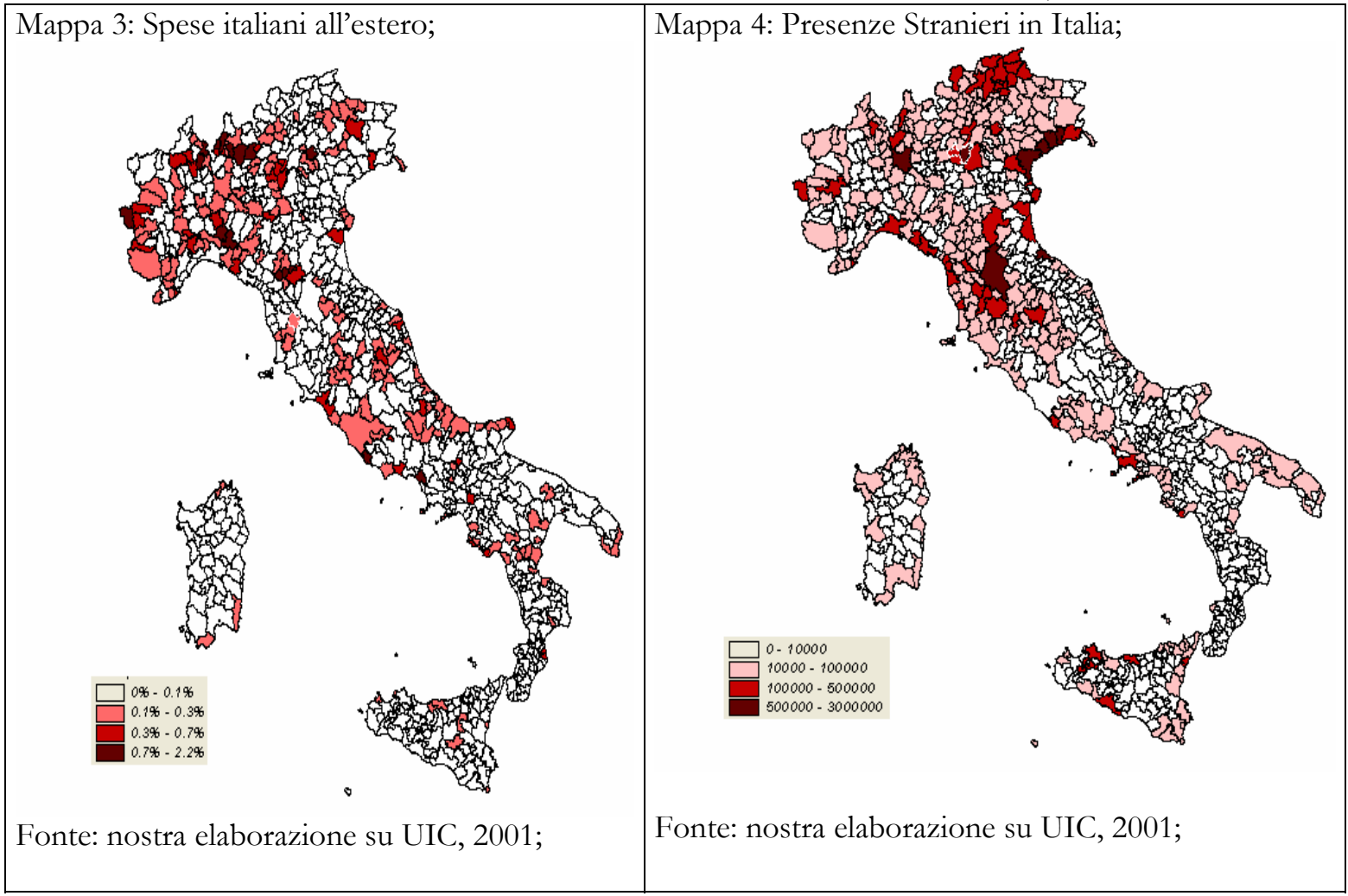




\section{APPENDICE 5.1: L'INDICE INVERSO HERFINDHAL-HIRSCHMAN (IHHI)}

L'indice di Herfindhal è una semplice misura di varianza comunemente usata negli studi di concentrazioni di mercato. E' calcolato come la somma del quadrato della quota di mercato di ogni impresa. Per esempio, per un mercato di quattro imprese con una quota di mercato di $20 \%, 20 \%$, $15 \%$ e $45 \%$, the HHI è $2600\left(20^{2}+20^{2}+15^{2}+45^{2}\right)$. L'indice HHI può variare tra un minimo di 0 ed un massimo di $10^{\prime} 000$.

Le analisi ed i contributi sugli spillovers spaziali (Glaeser et al. 1992, De Lucio, et al. 2002, Paci, Usai, 1999, etc.) utilizzano l'indice inverso di HHI come un indice di diversificazione della struttura industriale di un luogo in modo da rappresentare le economie di diversità $a$ la Jacobs. Più grande è questo tasso, maggiore è la diversificazione della struttura industriale nell'unità territoriale considerata. filiera:

Nel nostro caso la variabile Filiere, per esempio, è un indice IHHI calcolato all'interno della

$$
\text { Filiere }=1 / \sum_{j}\left[\frac{E_{i s}^{1991}}{E_{s}^{1991}}\right]^{2}
$$

Questo indica il grado di presenza della filiera a livello locale, maggiore è l'indice maggiore è la presenza degli anelli della filiera a livello locale.

\section{APPENDICE 5.2: I COMUNI INSERITI NELLA VARIABILE PACA}

Elenco 1: Siti patrimonio UNESCO dell'Umanità.

\begin{tabular}{|lcc|}
\hline Sito & anno & comune \\
Arte Rupestre della Val Camonica & 1979 & - \\
Santa Maria delle Grazie e il Cenacolo & 1980 & - \\
Centro storico di Firenze & 1982 & Firenze \\
Venezia e la sua Laguna & 1987 & Venezia \\
Pisa, Piazza del Duomo & 1987 & Pisa \\
Centro storico di San Gimignano & 1990 & San Gimignano \\
I Sassi di Matera & 1993 & Matera \\
Vicenza, Città del Palladio & 1994 & Vicenza \\
Centro storico di Siena & 1995 & Siena \\
Centro storico di Napoli & 1995 & Napoli \\
Insediamento industriale di Crespi d'Adda & 1995 & Capriate san Gervasio \\
Centro storico di Ferrara & 1995 & Ferrara \\
Castel del Monte & 1996 & Andria \\
Trulli di Alberobello & 1996 & Alberobello \\
Monumenti paleocristiani di Ravenna & 1996 & Ravenna \\
Centro storico di Pienza & 1996 & Pienza \\
Reggia di Caserta, il Parco, l'acquedotto Va & 1997 & Caserta \\
Residenze Sabaude & 1997 & Torino \\
Padova, l'Orto botanico & 1997 & Padova \\
Portovenere, Cinque Terre e Isole (Palmaria, & 1997 & Portovenere \\
Modena: Cattedrale, Torre Civica e Piazza Gr & 1997 & Modena \\
Aree archeologiche di Pompei, Ercolano e Tor & 1997 & Torre Annunziata \\
Costiera Amalfitana & 1997 & Salerno \\
Area Archeologica di Agrigento & 1997 & Agrigento \\
Enna. Piazza Armerina, La Villa del casale & 1997 & Enna \\
Villaggio Nuragico di Barumini & 1997 & Barumini \\
Parco Nazionale del Cilento & 1998 & - \\
\hline
\end{tabular}


A p pendice

\begin{tabular}{|lcc|}
\hline Centro storico di Urbino & 1998 & Urbino \\
Zona Archeologica e Basilica Patriarcale di & 1998 & Aquileia \\
Villa Adriana (Tivoli) & 1999 & Tivoli \\
Isole Eolie & 2000 & Eolie \\
Assisi, la Basilica di San Francesco e altri & 2000 & Assisi \\
Città di Verona & 2000 & Verona \\
Villa d'Este (Tivoli) & 2001 & Tivoli \\
Città Barocche del Val di Noto & 2002 & Siracusa \\
Sacri Monti del Piemonte e della Lombardia & 2003 & Piu comuni \\
Val d'Orcia & 2004 & Tre comuni \\
Necropooli etrusca di Cerveteri e Tarquinia & 2004 & Tarquina \\
Centro storico di Roma & 1980 & Roma \\
\hline
\end{tabular}

Fonte: Unesco.

\section{Elenco 2 - I Parchi nazionali in Italia;}

Parco nazionale dell'Abruzzo,Lazio e Molise: Alfedena, Barrea, Bisegna, Civitella Alfedena, Gioia dei Marsi, Lecce nei Marsi, , Opi, Ortona dei Marsi, Pescasseroli, Scanno, Villavallelonga, Villetta Barrea, Alvito, Campoli Appennino, Picinisco, San Biagio Saracinisco, San Donato Val di Comino, Settefrati, Castel San Vincenzo, Filignano, Pizzone, Rocchetta a Volturno, Scapoli., La Maddalena: Porto Torres, LA maddallena, , Parco nazionale toscano: Isola del Giglio, Campo nell'Elba, Capoliveri, Capraia Isola, Livorno, Marciana, Marciana Marina, Porto Azzurro, Portoferraio, Rio Marina, Rio nell'Elba, , Parco nazionale dell'aspromonte: Africo, Antonimina, Bagaladi, Bova, Bruzzano Zeffirio, Canolo, Cardeto, Careri, Ciminà, Cinquefrondi, Cittanova, Condofuri, Cosoleto, Delianuova, Gerace, Mammola, Molochio, Oppido Mamertina, Palizzi, Platì, Reggio Calabria, Roccaforte del Greco, Roghudi, Samo, San Giorgio Morgeto, San Lorenzo, San Luca, San Roberto, Santa Cristina d'Aspromonte, Sant'Agata del Bianco, Sant'Eufemia d'Aspromonte, Santo Stefano in Aspromonte, Scido, Scilla, Sinopoli, Staiti, Parapodio, , Parco nazionale della Calabria: Acri, Albi, Aprigliano, Bocchigliero, Celico, Corigliano Calabro, , Cotronei, , Longobucco, Magisano, , Mesoraca, Pedace, , Petilia Policastro, , Petronà, , S. Giovanni in Fiore, , Savelli, Serra Pedace, , Sersale, Spezzano della Sila, Spezzano Piccolo, Taverna,, Zagarise, Circeo, , Parco nazionale del Lazio: Latina, Sabaudia, San Felice Circeo, Ponza, , Parco nazionale del Cilento: Campani, Agropoli, Aquara, Ascea, Auletta, Bellosguardo, Buonabitacolo, Camerota, Campora, Cannalonga, Capaccio, Casalbuono, Casaletto Spartano, Casal Velino, Caselle in Pittari, Castelcivita, Castellabate, Castelnuovo Cilento, Castel San Lorenzo, Celle di Bulgheria, Centola, Ceraso, Cicerale, Controne, Corleto Monforte, Cuccaro Vetere, Felitto, Futani, Gioi, Giungano, Laureana Cilento, Laurino, Laurito, Lustra, Magliano Vetere, Moio della Civitella, Montano Antilia, Montecorice, Monteforte Cilento, Monte San Giacomo, Montesano sulla MarcelIana, Morigerati, Novi Velia, Omignano, Orria, Ottati, Perdifumo, Perito, Petina, Piaggine, Pisciotta, Polla, Pollica, Postiglione, Roccadaspide, Roccagloriosa, Rofrano, Roscigno, Sacco, San Giovanni a Piro, San Mauro Cilento, San Mauro la Bruca, San Pietro al Tanagro, San Rufo, Santa Marina, Sant'Angelo a Fasanella, Sant'Arsenio, Sanza, Sassano, Serramezzana, Sessa Cilento, Sicignano degli Alburni, Stella Cilento, Stio, Teggiano, Torre Orsaia, Tortorella, Trentinara, Valle dell'Angelo, Vallo della Lucania, Parco nazionale dolomiti Bellunesi, , Parco nazionale del Veneto: Belluno, Cesiomaggiore, Feltre, Forno di Zoldo, Gosaldo, La Valle Agordina, Longarone, Pedavena, Ponte nelle Alpi, Rivamonte, San Gregorio nelle Alpi, Santa Giustina, Sedico, Sospirolo, Sovramonte, , , Parco nazionale dell Cinque terre: Monterosso al Mare, Riomaggiore, Vernazza, , , Monti falterona:Bagno di Romagna, Portico - San Benedetto, Premilcuore, Santa Sofia, Tredozio, Bibbiena, Chiusi Della Verna, Poppi, Pratovecchio, Stia, Londa, San Godenzo, , Parco nazionale del Gargano: Puglia: Apricena, Cagnano Varano, Carpino, Ischitella, Isole Tremiti - San Nicola, Lesina, Manfredonia, Mattinata, Monte Sant'Angelo, Peschici, Poggio Imperiale, Rignano Garganico, Rodi Garganico, San Giovanni Rotondo, San Marco in Lamis, Sannicandro Garganico, Serracapriola, Vico del Gargano, Vieste, Parco nazionale del Gran paradiso: Piemonte (Torino).Ceresole Reale, Locana, Noasca, Ribordone, Ronco Canavese, Valprato Soana. Valle d'Aosta (Aosta) Aymavilles, Cogne, Introd, Rhêmes N. Dame, Rhêmes S. Georges, Valsavarenche, 
Villeneuve, Parco nazionale del GRAN SASSO: Abruzzo (L'Aquila) Barete, Barisciano, Cagnano Amiterno, Calascio, Campotosto, Capestrano, Capitignano, Carapelle Calvisio, Castel del Monte, Castelvecchio Calvisio, L'Aquila, Montereale, Ofena, Pizzoli, Santo Stefano di Sessanio, Villa Santa Lucia. (Teramo): Arsita, Campli, Castelli, Civitella del Tronto, Cortino, Crognaleto, Fano Adriano, Isola del Gran Sasso, Montorio al Vomano, Pietracamela, Rocca Santa Maria, Torricella Sicura, Tossicia, Valle Castellana., (Pescara): Brittoli, Bussi sul Tirino, Carpineto della Nora, Castiglione a Casauria, Civitella Casanova, Corvara, Farindola, Montebello di Bertona, Pescosansonesco, Villa Celiera. Lazio: (Rieti): Accumoli, Amatrice. Marche: (Ascoli Piceno): Acquasanta Terme, Arquata del Tronto, MAJELLLA, Abruzzo, (Chieti), Civitella Messer Raimondo, Fara San Martino, Gamberale, Guardiagrele, Lama dei Peligni, Lettopalena, Montenerodomo, Rapino, Palena, Palombaro, Pennapiedimonte, Pizzoferrato, Pretoro, Taranta Peligna, (L'Aquila), Ateleta, Campo di Giove, Cansano, Corfinio, Pacentro, Pescocostanzo , Pratola Peligna, Rivisondoli, Roccacasale, Roccapia, Roccaraso, Sulmona, (Pescara), Abbateggio, Bolognano, Caramanico Terme, Lettomanoppello, Manoppello, Popoli, Roccamorice, Salle, Sant'Eufemia a Majella, San Valentino in Abruzzo Citeriore, Serramonacesca, Tocco da Casauria, , , Parco nazionale dei Monti sibillini: , Marche, (Ascoli Piceno), Amandola, Arquata del Tronto, Montefortino, Montegallo, Montemonaco, (Macerata), Acquacanina, Bolognola, Castelsantangelo sul Nera, Cessapalombo, Fiastra, Fiordimonte, Pievebovigliana, Pievetorina, San Ginesio, Ussita, Visso, Umbria, (Perugia), Norcia, Preci, , Parco nazionale del POLLINO: , Basilicata, (Matera), San Giorgio Lucano, Valsinni, (Potenza), Calvera, Carbone, Castelluccio Inferiore, Castelluccio Superiore, Castelsaraceno, Castronuovo S. Andrea, Cersosimo, Chiaromonte, Episcopia, Fardella, Francavilla in Sinni, Latronico, Lauria, Noepoli, Rotonda, San Costantino Albanese, San Paolo Albanese, San Severino Lucano, Senise, Teana, Terranova di Pollino, Viggianello, Calabria, (Cosenza), Acquaformosa, Aieta, Alessandria del Carretto, Belvedere Marittimo, Buonvicino, Castrovillari, Cerchiara di Calabria, Civita, Francavilla Marittima, Frascineto, Grisolia, Laino Borgo, Laino Castello, Lungro, Maierà, Morano Calabro, Mormanno, Mottafollone, Orsomarso, Papasidero, Plataci, Praia a Mare, San Basile, San Donato di Ninea, Sangineto, San Lorenzo Bellizzi, San Sosti, Sant'Agata d'Esaro, Santa Domenica Talao, Saracena, Tortora, Verbicaro, , Parco nazionale dello STELVIO: , Settore Lombardo, Livigno , Valdidentro, Bormio, Valfurva, Valdisotto, Sondalo , Vezza d'Oglio, Vione, Temù, Ponte di Legno, Settore Alto Atesino, Ultimo, Laces, Martello, Silandro, Lasa, Prato allo Stelvio, Stelvio , Tubre, Malles Venosta, Glorenza, Sluderno

Fonte: www.federparchi.it

\section{Elenco 3: Le Blueflags delle spiaggie italiane;}

Acciaroli e Pioppi di Pollica , Alba Adriatica , Anzio , Ascea, Bergeggi, Bibbona , Bibione , Bordighera , Calata Turchina e Molo Sud di San Vito Chietino , Campomarino , Camporosso , Cannobbio, Castagneto Carducci, Castellabate, Castiglioncello e Vada di Rosignano Marittimo , Castiglione della Pescaia, Catanzaro Lido , Cattolica, Celle Ligure, Cervia , Cesenatico , Chiavari, Cirò Marina , Civitanova Marche, Comacchio , Cupra Marittima, Fano , Follonica , Fornaci e Natarella di Savona , Forte dei Marmi , Fossacesia Marina , Francavilla al Mare (zone Centro e Alcione) , Gabicce Mare, Gaeta , Gatteo a Mare, Ginosa , Grado, Grottammare, Jesolo , La Rena Bianca di S. Teresa di Gallura , Lavagna, Lerici, Lido Marinello e San Teodoro di Marsala , Lignano Sabbiadoro , Maratea , Marina di Gioiosa Jonica , Marina e Principina di Grosseto, Martinsicuro, Menfi , Misano Adriatico , Moneglia, Numana Bassa, Ostuni , Palinuro , Peschici , Pietrasanta , Pisciotta , Poetto di Quartu S. Elena, Porto Galeazzi e Viale Gennari di Sirmione, Porto San Giorgio, Positano , Raganzino e Pietrenere di Pozzallo , Rimini , Rocca San Giovanni , Roccella Jonica , Roseto Capo Spulico , Roseto degli Abruzzi, S. Benedetto del Tronto , S. Salvo , Sabaudia , San Mauro Pascoli , Sapri, Scalette e Salsello di Bisceglie , Scanno, Scialmarino e Pugnochiuso di Vieste , Scilla , Senigallia, Sirolo , Sperlonga , Termoli , Tirrenia , Torre di Barì di Barisardo , Tortoreto , Varazze, Vasto , Viareggio.

Fonte: www.blueflags.com 
Elenco 4 - Le destinazioni sciistiche;

Abetone, Alagna, Alleghe, Antey-Saint-Andre', Aosta, Aprica, Artogne, Asiago, Bardonecchia, Bormio, Brenta, Bressanone, Campodolcino, Canazei, Carona, Caspoggio, Castello-Molina Di Fiemme, Cesana Torinese, Challand-Saint-Anselme, Chambave, Chiesa In Valmalenco, Claviere, Cogne, Cortina D'ampezzo, Corvara, Courmayeur, Dimaro, Fai Della Paganella, Falcade, Foppolo, Gressoney-La-Trinite', La Thuile, Limone Piemonte, Livigno, Macugnaga, Mezzana, Morgex, Pila, Pinzolo, Ponte Di Legno, Ponte Gardena, Pont-Saint-Martin, Roccaraso, Saint-Nicolas, Santa Cristina Valgardena, Sauze D'oulx, Selva Di Val Gardena, Senales, Sestriere, Valfurva, Valtournenche.

Fonte: Touring Club Italia; 